\title{
Experimental and CFD Investigation of the Mixing of MMA \\ Emulsion Polymerization in a Stirred Tank Reactor
}

\author{
By \\ SHIDEH FATHI ROUDSARI \\ M.Sc., Tarbiat Modares University, Tehran, Iran, 2001 \\ B.Sc., Amir Kabir University of Technology, Tehran, Iran, 1996
}

\author{
A Dissertation \\ Presented to Ryerson University \\ in Partial Fulfillment of the Requirements for the Degree of \\ Doctor of Philosophy (PhD) \\ in the Program of Chemical Engineering
}

Toronto, Ontario, Canada, 2015

Copyright $(2015$ by Shideh Fathi Roudsari 


\section{$\underline{\text { Author's Declaration }}$}

I hereby declare that I am the sole author of this dissertation. This is a true copy of the dissertation, including any required final revisions, as accepted by my examiners.

I authorize Ryerson University to lend this dissertation to other institutions or individuals for the purpose of scholarly research.

I further authorize Ryerson University to reproduce this dissertation by photocopying or by other means, in total or in part, at the request of other institutions or individuals for the purpose of scholarly research.

I understand that my dissertation may be made electronically available to the public.

Author's Signature

Shideh Fathi Roudsari 


\author{
ABSTRACT \\ Experimental and CFD Investigation of the Mixing of MMA Emulsion Polymerization in a \\ Stirred Tank Reactor \\ Shideh Fathi Roudsari \\ Doctor of Philosophy, Chemical Engineering Department \\ Ryerson University, Toronto, 2015
}

Although significant advances have been achieved in emulsion polymerization in recent decades, the effect of mixing on this type of polymerization has not been fully delineated yet. In fact, mixing plays a significant role in the performance of an emulsion polymerization reaction. For instance, in case of a very low agitation rate, larger droplets are generated and phase separation, which limits the diffusion mechanism, may occur. In contrast, vigorous agitation can result in reduced nucleation of particles. Therefore, the main objective of this study is to investigate the impact of mixing parameters (e.g. impeller speed, impeller type, impeller number, and baffles) on the monomer conversion, the polymer average molecular weight, particle size and size distributions, transition glass temperature, and number of particles.

To achieve this objective, the emulsion polymerization of methyl methacrylate (MMA) was carried out in a lab-scale reactor equipped with a top-entry agitator, 4 wall baffles, a $\mathrm{U}$ shaped cooling coil, and a temperature controller. To analyze the reactive flow inside the polymerization reactor, a novel computational fluid dynamics (CFD) model was developed. The multiple reference frames (MRF) technique, $k-\varepsilon$ model, and mixture model approach were employed to model the impeller rotation, turbulence, and multiphase flow, respectively. The particle number density distribution within the reactor was also estimated by means of the population balance 
approach, which employs a discrete method to describe the nucleation and growth of the polymer particles. The experimental data and CFD results showed that the installation of the baffles enhanced the particle size and molecular weight but reduced the conversion and particle number. The number density achieved using the Rushton impeller was higher than that for the pitched blade impeller. The results revealed that the effect of the impeller speed on the characteristics of the polymer attained using the pitched-blade turbine was more prominent than that for the Rushton turbine. It was also found that the impact of the impeller speed on the polymer characteristics was much more pronounced for the double pitched-blade turbines rather than for the double Rushton turbines. 


\section{ACKNOWLEDGMENTS}

I wish to thank my first advisor, Professor Farhad Ein-Mozaffari for his continuous support of my $\mathrm{PhD}$ study and research, for his leadership, direction, encouragement, thoughtfulness and patience throughout this work. I express my appreciation to my co-advisor, Professor Ramdhane Dhib for his guidance, inspiration, attention and carefulness. I benefited from immense knowledge of my both advisors and thank them for giving me the freedom to move on and mix the two interesting scientific fields of their expertise. I would also like to take this opportunity to thank my committee members, Professor Adel Al Taweel, Professor Ziad Saghir, Professor Simant R. Upreti, and Professor Yaser Dahman for their time and consideration.

I acknowledge the assistance of the engineering staffs, Mr. Ali Hemmati, Mr. Daniel Boothe, and Mr. Tondar Tajrobekar, and also the help of administration staff, Mrs. Leah Rogan, Mrs. Isabella Fernandes, and Mrs. Louise Lichacz in the Chemical Engineering Department at Ryerson University. Special thanks to Professor Daniel Foucher (Department of Chemistry), Professor Alexander Penlidis, Professor Neil McManus and Mr. Ralph Dickhout (University of Waterloo), Dr. Lida Mahal (Cosmetica labs Inc.), Dr. Stephan Drappel and Mr. Paul Gerroir (Xerox Research Centre) for valuable discussions and providing the test facilities. I wish to thank the former and current student colleagues at Ryerson University for their cherished friendship throughout these years. I acknowledge Natural Sciences and Engineering Research Council of Canada (NSERC), Queen Elizabeth II Graduate Scholarships in Science and Technology (QEII GSST), and Ryerson Graduate Scholarship for the financial support during this work. I also acknowledge the HPCVL for providing the high performance computing facilities. Finally my everlasting love goes to my dear husband, Sadegh for his kindheartedness, compassion and support and to my dear son, Arya for his goodness and patience throughout my years of study. 


\section{TABLE OF CONTENTS}

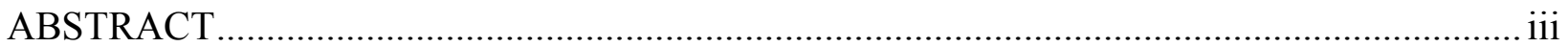

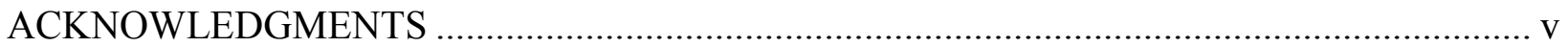

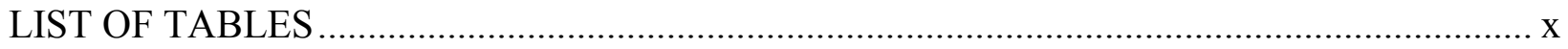

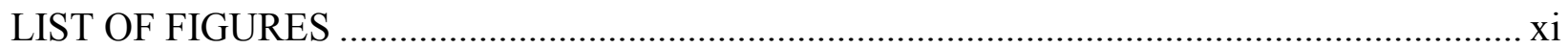

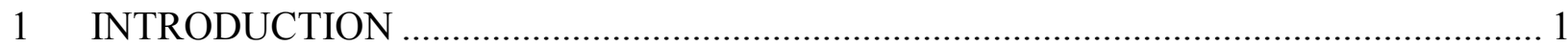

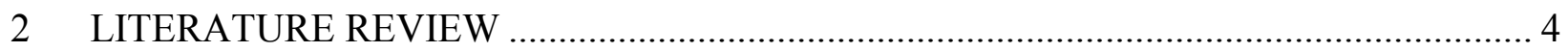

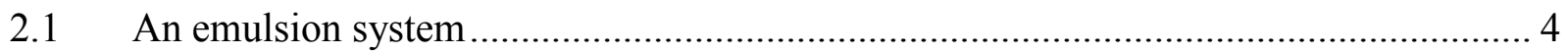

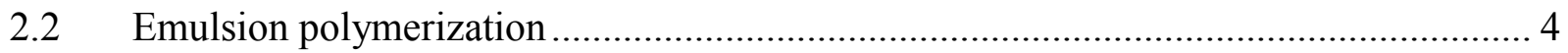

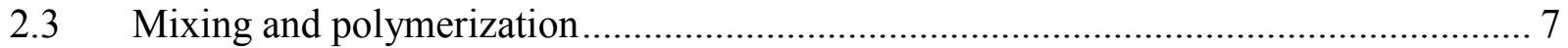

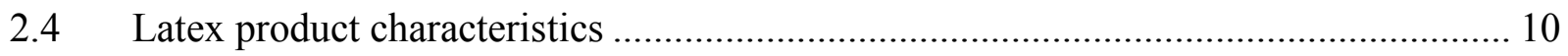

2.5 Nucleation and growth and emulsion polymerization ................................................ 11

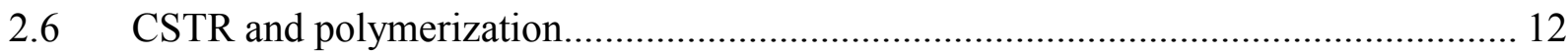

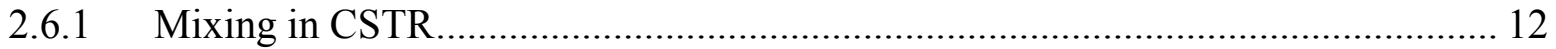

2.6.2 Effect of reactants flow rate on CSTR ............................................................ 13

2.7 Effect of solvent and gel effect on solution polymerization ..................................... 13

2.8 Methyl methacrylate polymerization ................................................................ 14

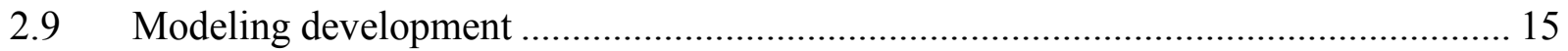

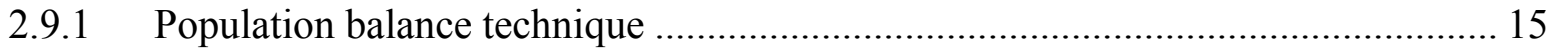

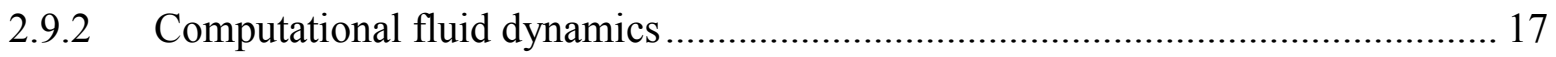




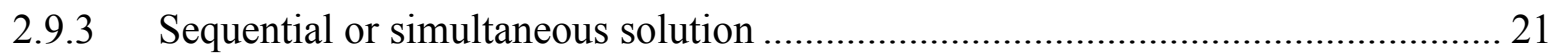

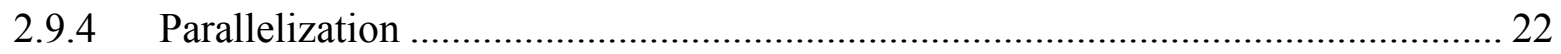

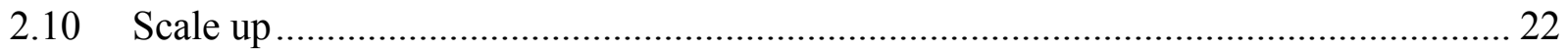

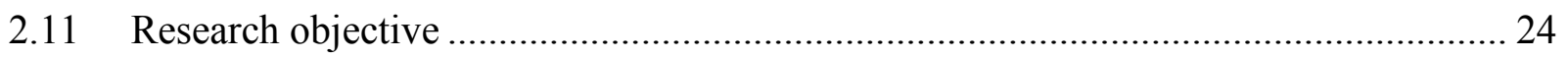

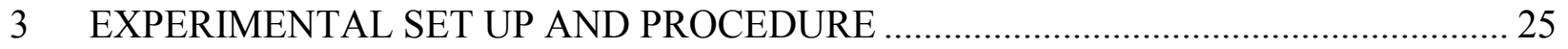

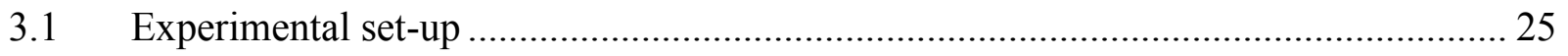

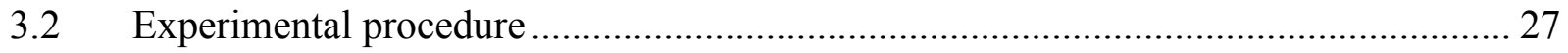

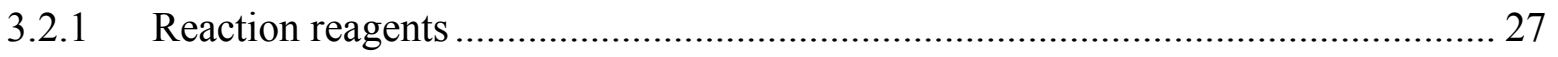

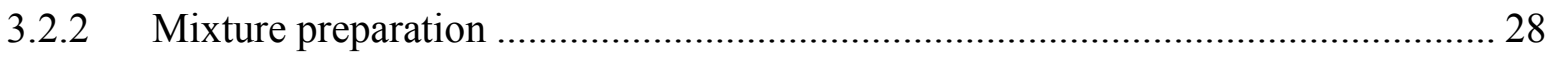

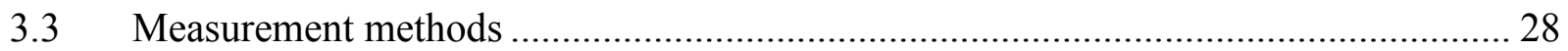

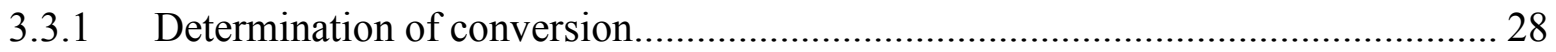

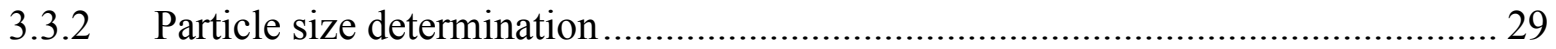

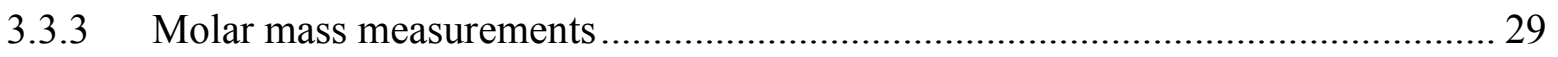

3.3.4 Glass transition temperature determination ...................................................... 30

3.3.5 Scanning electron microscopy (SEM) micrographs ............................................. 30

3.4 Experimental design............................................................................................ 31

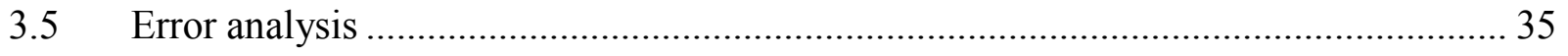

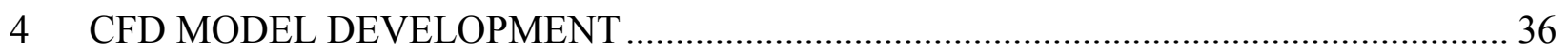

4.1 Modeling of the emulsion polymerization system .................................................... 37

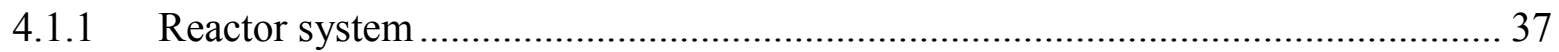


4.1.2 Mathematical model............................................................................... 37

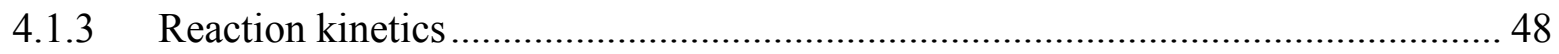

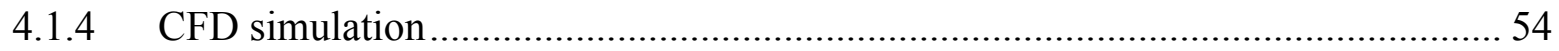

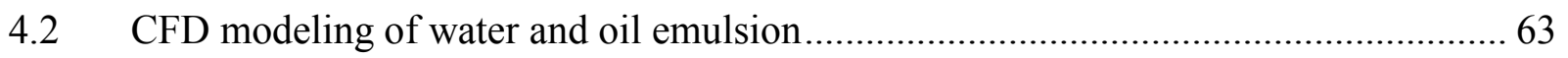

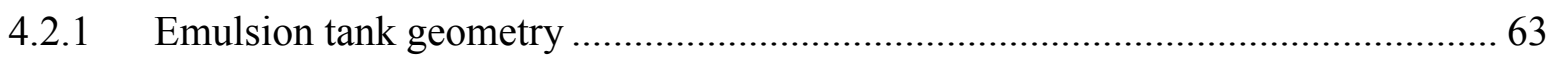

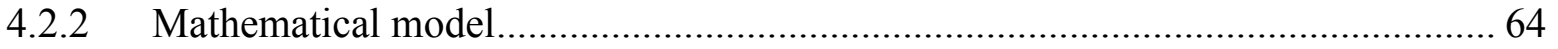

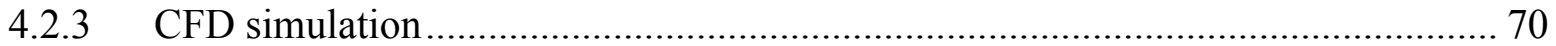

4.3 Modeling of MMA polymerization in a CSTR ...................................................... 74

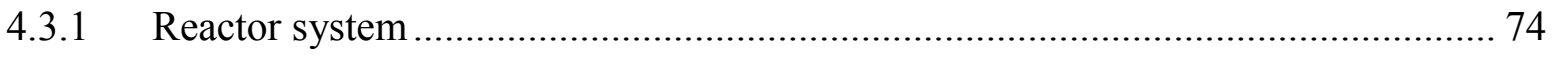

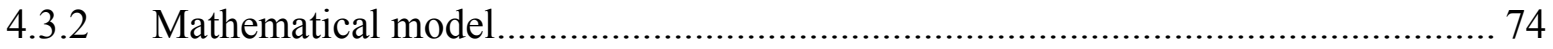

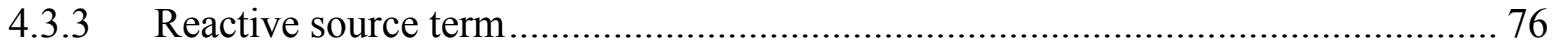

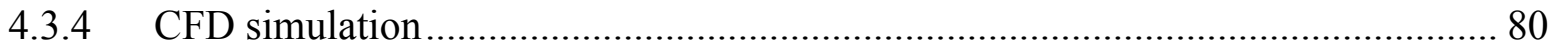

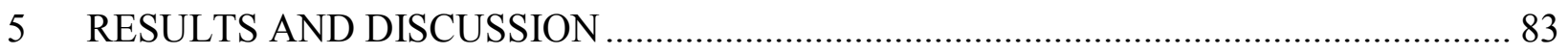

5.1 Mixing effect on emulsion polymerization in a batch reactor …............................ 85

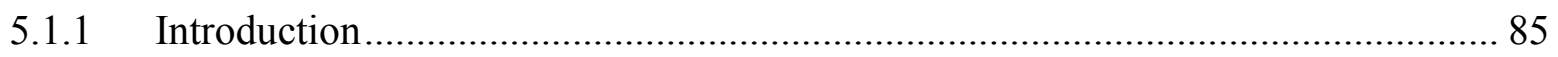

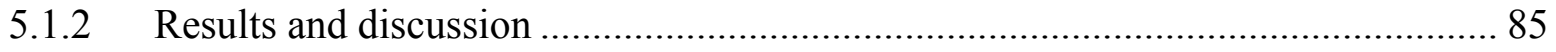

5.1.3 Concluding Remarks........................................................................... 102

5.2 Impact of impeller type on MMA emulsion polymerization in a batch reactor ........ 105

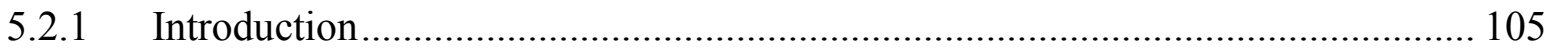

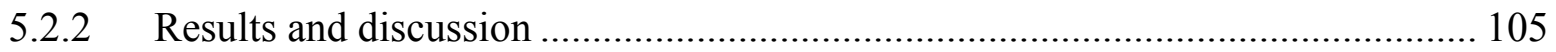


5.2.3 Concluding remarks

5.3 Analysis of mixing in emulsion polymerization of MMA.................................... 128

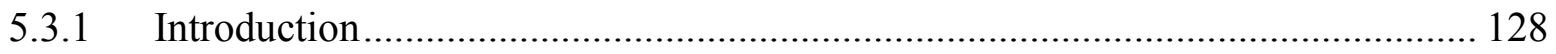

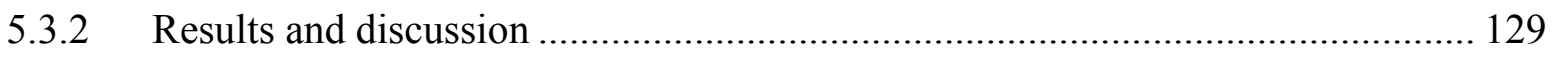

5.3.3 Concluding Remarks........................................................................ 144

5.4 CFD modeling of the mixing of water in oil Emulsions ..................................... 147

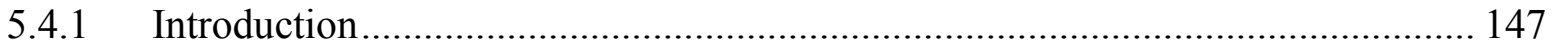

5.4.2 Results and discussion ....................................................................... 148

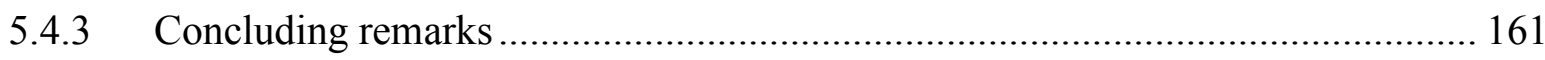

5.5 Use of CFD in modeling MMA solution polymerization in a CSTR ..................... 163

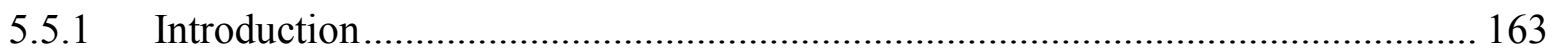

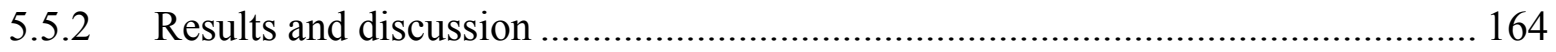

5.5.3 Concluding remarks .......................................................................... 177

6 OVERALL CONCLUSION AND RECOMMENDATIONS FOR FUTURE WORK ..... 178

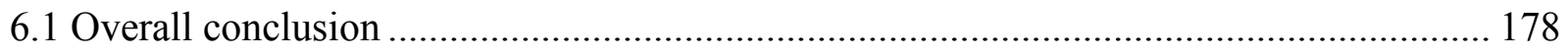

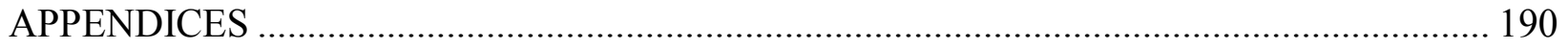

A.1 User defined function for MMA emulsion Polymerization ..................................... 190

A.2 Physical Properties of MMA solution polymerization ............................................ 193

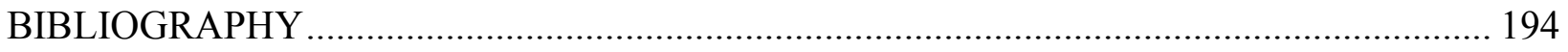

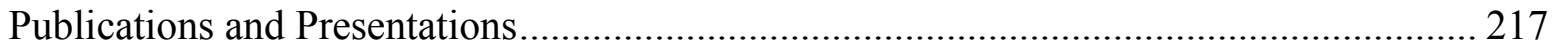




\section{LIST OF TABLES}

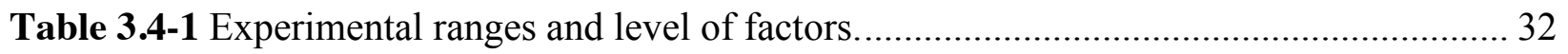

Table 3.4-2 Experimental design 1 of the experiments............................................................ 33

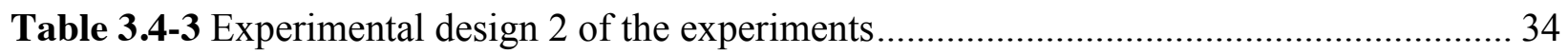

Table 4.1-1 Values of kinetic parameters for the emulsion polymerization............................... 50

Table 4.1-2 Diameter of the particle sizes used in the discrete method...................................... 55

Table 4.1-3 RMS values for the reactor equipped with the pitched blade impeller at the speed of $350 \mathrm{rpm}$ for different number of cells 61

Table 4.1-4 RMS values for the reactor equipped with the Rushton impeller at the speed of 350 $\mathrm{rpm}$ for different number of cells at the location $x=0.03, y=0.0$, and $z=0.08$ to $0.105 \mathrm{~m}$.......... 62

Table 4.2-1 Physical properties of fluids at 20 $\mathrm{C}$ and $1 \mathrm{~atm}$ (from Boxall et al., 2010)............ 64

Table 4.2-2 Diameter of the drop sizes used in discrete method. .............................................. 73

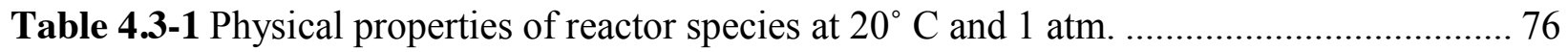

Table 4.3-2 Values of kinetic parameters for free radical polymerization of MMA................... 78

Table 5.1-1 Final particle numbers at different reactor conditions........................................... 90

Table 5.2-1 ANOVA results for the final conversion response. ............................................. 123

Table 5.2-2 ANOVA results for the final molecular weight response....................................... 124

Table 5.2-3 ANOVA results for the final particle size response. ........................................... 125

Table 5.3-1 Volume average shear rates computed using the CFD model ............................... 136

Table 5.3-2 Flow numbers computed using the CFD model for the pitched blade and the Rushton impellers at different impeller speeds in the reactors equipped with the baffles. 139 


\section{LIST OF FIGURES}

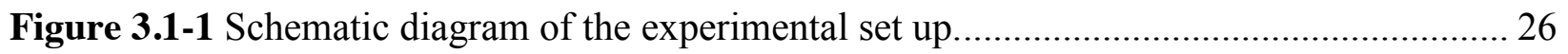

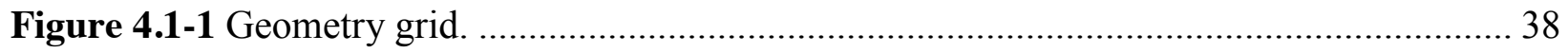

Figure 4.1-2 Monomer mass fraction convergence at the function of iteration.......................... 56

Figure 4.1-3 Grid independency study through axial velocity profile using the pitched blade

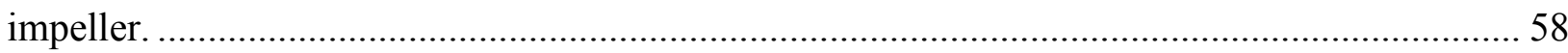

Figure 4.1-4 Grid independency study through turbulent kinetic energy profile using the pitched

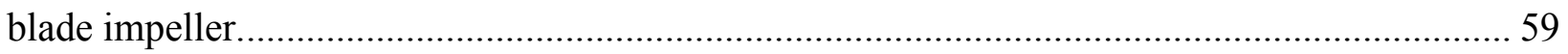

Figure 4.1-5 Grid independency study through polymer phase volume fraction profile using the

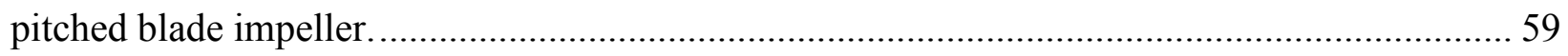

Figure 4.1-6 Grid independency study through radial velocity profile using the Rushton impeller.

Figure 4.1-7 Grid independency study through turbulent kinetic energy profile using the Rushton impeller.

Figure 4.1-8 Grid independency study through polymer phase volume fraction profile using the Rushton impeller. 61

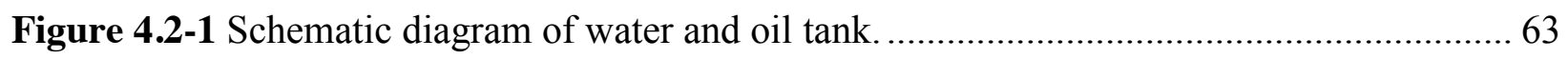

Figure 4.2-2 Geometry grid of water and oil emulsion system. ............................................... 70

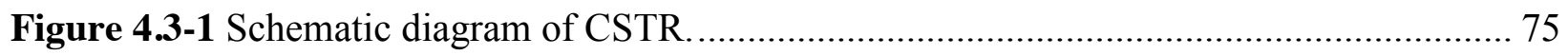

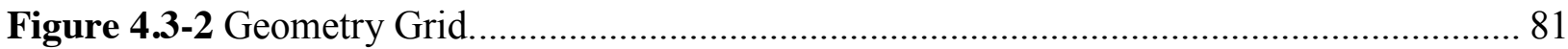

Figure 5.1-1 Conversion as a fucntion of time at the speed of 20,100, 250 and $350 \mathrm{rpm}$, with and without baffles at the isothremal reaction temperature of a) $50^{\circ} \mathrm{C}$ and b) $60^{\circ} \mathrm{C}$. 88 
Figure 5.1-2 Mean particle size as a fucntion of time conversion at the speed of 20, 100, 250 and $350 \mathrm{rpm}$, with and without baffles at the isothremal reaction temperature of a) $50^{\circ} \mathrm{C}$ and b) $60^{\circ} \mathrm{C}$. 92

Figure 5.1-3 Average weight molecular weight as a fucntion of time conversion at the speed of 20, 100, 250 and $350 \mathrm{rpm}$, with and without baffles at the isothremal reaction temperature of a) $50^{\circ} \mathrm{C}$ and b) $60^{\circ} \mathrm{C}$. 95

Figure 5.1-4 Polydispersity of polymers as a fucntion of time conversion at the speed of 20, 100, 250 and $350 \mathrm{rpm}$, with and without baffles at the isothremal reaction temperature of a) $50^{\circ} \mathrm{C}$ and b) $60^{\circ} \mathrm{C}$. 97

Figure 5.1-5 Effect of impeller speed on a) conversion, b) mean particle size and c) average weight molecular weight after $130 \mathrm{~min}$. 99

Figure 5.1-6 Particle size distribution at $60^{\circ} \mathrm{C}$ and the impeller speed of $250 \mathrm{rpm}$, reactors with and without baffles. 100

Figure 5.1-7 RI response of GPC analysis at $60^{\circ} \mathrm{C}$, reactor with baffles and the impeller speed of $250 \mathrm{rpm}$. 102

Figure 5.1-8 Scan Electron Microscopy images at $60^{\circ} \mathrm{C}$, reactor with baffles and the impeller speed of a) $100 \mathrm{rpm}$, b) $250 \mathrm{rpm}$ and c) $350 \mathrm{rpm}$. 103

Figure 5.2-1 Monomer conversion versus reaction time for a) pitched blade turbine and b) Rushton impeller at the speeds of 100-350 rpm using both single and double impellers. 107

Figure 5.2-2 Final monomer conversion versus impeller speed at reaction time of 130 min for single/double pitched blade and Rushton impellers. 110 
Figure 5.2-3 Weight average molecular weight versus monomer conversion for a) pitched blade turbine and b) Rushton impeller at the speeds of 100-350 rpm using both single and double impellers.

Figure 5.2-4 Final weight average molecular weight versus impeller speed at reaction time of 130 min for single/double pitched blade and Rushton impellers. 114

Figure 5.2-5 Final polymer poly dispersity versus impeller speed at reaction time of 130 min for single/double pitched blade and Rushton impellers. 115

Figure 5.2-6 Mean particle size versus monomer conversion for a) pitched blade turbine and b) Rushton impeller at the speeds of 100-350 rpm using both single and double impellers. 117

Figure 5.2-7 Final mean particle size versus impeller speed at reaction time of 130 min for single/double pitched blade and Rushton impellers.

Figure 5.2-8 Particle size distribution at reaction times of 25, 75 and 130 min using pitched blade impeller and Rushton turbine at the impeller speed of $250 \mathrm{rpm}$. 120

Figure 5.2-9 Heat flow versus temperature for final polymer at the impeller speeds of 100-350 rpm using pitched blade turbine and Rushton impeller. 121

Figure 5.2-10 SEM image of final polymer product using Rushton turbine at impeller speed 250 rpm. 126

Figure 5.3-1 Monomer conversion versus impeller speed at the reaction time of $130 \mathrm{~min}$ for the pitched blade impeller and the reactors with baffles and without baffles. 131

Figure 5.3-2 Contours of the first phase volume fraction on the top surface in the reactor equipped with the pitched blade impeller rotating at $250 \mathrm{rpm}$ : a) with baffles and b) without baffles. 132 
Figure 5.3-3 Number density distribution of particles computed through the CFD model for the pitched blade impeller in the reactor with the baffles at the rotational speeds of $20,100,250$, and $350 \mathrm{rpm}$. 133

Figure 5.3-4 Number density distribution of particles computed through the CFD model for the pitched blade impeller in the reactor without baffles at the rotational speeds of $20,100,250$, and $350 \mathrm{rpm}$. 134

Figure 5.3-5 Sauter mean particle size versus impeller speed at the reaction time of 130 min for the pitched blade impeller and the reactors with baffles and without baffles. 136 Figure 5.3-6 Contours of axial velocity of mixture $(\mathrm{m} / \mathrm{s})$ generated by the pitched blade impeller at the impeller speed of $350 \mathrm{rpm}$ in the reactor a) with baffles and b) without baffles. 137

Figure 5.3-7 Monomer conversion versus impeller speed at the reaction time of $130 \mathrm{~min}$ for the pitched blade and the Rushton impellers in the reactor with baffles. 140

Figure 5.3-8 Number density distriubtion of particles computed through the CFD model for the Rushton impeller at the speed of 100,250 , and $350 \mathrm{rpm}$ in the reactor with baffles. 142

Figure 5.3-9 Sauter mean particle size versus impeller speed at the reaction time of 130 min for the pitched blade and the Rushton impellers in the reactor with baffles. 143

Figure 5.3-10 Contours of the second phase volume fraction in the reactor equipped with the baffles at the speed of $250 \mathrm{rpm}$ for the: a) Rushton impeller and b) pitched blade impeller...... 145 Figure 5.4-1 Cumulative probability drop size distribution versus droplet diameter at impeller speeds of: $\bullet 300, \mathbf{\square} 400$ and $\boldsymbol{\Delta} 600 \mathrm{rpm}$ and continuous phase of Conroe oil with 15 vol\% water at $20^{\circ} \mathrm{C}$ 149

Figure 5.4-2 Logarithm of number density of droplets at impeller speed of a) 300 rpm, b) 400 rpm and c) $600 \mathrm{rpm}$ in Conroe oil and $15 \mathrm{vol} \%$ water at $20^{\circ} \mathrm{C}$. 151 
Figure 5.4-3 Contour of turbulent kinetic energy $\left(\mathrm{m}^{2} / \mathrm{s}^{2}\right)$ at different impelle speeds: a) $300 \mathrm{rpm}$, b) $400 \mathrm{rpm}$, and c) $600 \mathrm{rpm}$ in Conroe oil and $15 \mathrm{vol} \%$ water at $20^{\circ} \mathrm{C}$. 152

Figure 5.4-4 Contour of water volume fraction at different impeller speeds: a) 300 rpm, b) 400 rpm, and c) $600 \mathrm{rpm}$ in Conroe oil and $15 \mathrm{vol} \%$ water fraction at $20^{\circ} \mathrm{C}$. 153

Figure 5.4-5 Effect of the continuous phase type ( $\bullet$ Troika oil and $\bullet$ Conroe oil) on the cumulative probability drop size distribution at $300 \mathrm{rpm}$ and $15 \mathrm{vol} \%$ water at $20{ }^{\circ} \mathrm{C}$. 154

Figure 5.4-6 Effect of the continuous phase type on logarithm of number density of droplets at $300 \mathrm{rpm}$ and $15 \mathrm{vol} \%$ water at $20^{\circ} \mathrm{C}$ 155

Figure 5.4-7 Contour of turbulent kinetic energy $\left(\mathrm{m}^{2} / \mathrm{s}^{2}\right)$ at $300 \mathrm{rpm}$ and the continuous phase of Troika oil with 15 vol $\%$ water. 157

Figure 5.4-8 Contour of water volume fraction at $300 \mathrm{rpm}$ and the continuous phase of Troika oil with 15 vol\% water. 158

Figure 5.4-9 Cumulative probability drop size distribution versus droplet diameter for: $\bullet 15$ vol\% and $\boldsymbol{\nabla} 25$ vol\% water at $300 \mathrm{rpm}$ with the continuous phase of Conroe oil at $20{ }^{\circ} \mathrm{C} \ldots \ldots 159$ Figure 5.4-10 Logarithm of number density at 15 vol\% and 25 vol\% water at $300 \mathrm{rpm}$ with the continuous phase of Conroe oil at $20^{\circ} \mathrm{C}$. 160

Figure 5.5-1 Effect of inlet solvent concentration on the conversion at the outlet at residence times of $\tau=45 \mathrm{~min}, \tau=60 \mathrm{~min}$, and $\tau=90 \mathrm{~min}$; impeller speed of $100 \mathrm{rpm}$ and $\mathrm{T}=65^{\circ} \mathrm{C}$. 165

Figure 5.5-2 Effect of temperature on the conversion at $\tau=30 \mathrm{~min}$ and $\tau=45 \mathrm{~min}$ at inlet $\phi_{m}=$ 0.7 and impeller speed of $100 \mathrm{rpm}$. 167

Figure 5.5-3 Contours of MMA monomer mass fraction at inlet monomer concentraton of: a) $\phi_{m}$ $=0.2$, b) $\phi_{m}=0.4$, c) $\phi_{m}=0.5$, d) $\phi_{m}=0.6$, and e) $\phi_{m}=0.7$ at residence time of $\tau=60 \mathrm{~min}$, impeller speed of $100 \mathrm{rpm}$, and $\mathrm{T}=65^{\circ} \mathrm{C}$ 169 
Figure 5.5-4 Contours of MMA monomer mass fraction at residence time of $\tau=45 \mathrm{~min}$, inlet monomer concentration of $\phi_{m}=0.7$, impeller speed of $100 \mathrm{rpm}$ and reaction temperatures of: a) $\left.\left.55^{\circ} \mathrm{C}, \mathrm{b}\right) 60^{\circ} \mathrm{C}, \mathrm{c}\right) 65^{\circ} \mathrm{C}$, and d) $70^{\circ} \mathrm{C}$ 170

Figure 5.5-5 Effect of impeller speed on the conversion as a function of the inlet MMA monomer concentration at: a) $\tau=45 \mathrm{~min}$, and b) $\tau=60 \mathrm{~min}$ and $\mathrm{T}=65^{\circ} \mathrm{C}$ 171

Figure 5.5-6 Contours of MMA monomer mass fraction at $\tau=60 \mathrm{~min}, \phi_{m}=0.6$ and impeller speed of: a) $50 \mathrm{rpm}$, b) $100 \mathrm{rpm}$, c) $200 \mathrm{rpm}$, d) $300 \mathrm{rpm}$, and e) $500 \mathrm{rpm}, \mathrm{T}=65^{\circ} \mathrm{C}$

Figure 5.5-7 Contour of MMA monomer mass fraction in planes perpendicular to shaft direction at locations of i) 0 , ii) $0.01 \mathrm{~m}$, iii) $0.02 \mathrm{~m}$, iv) $0.03 \mathrm{~m}, \mathrm{v}) 0.045 \mathrm{~m}$, vi) $0.06 \mathrm{~m}$, vii) $0.115 \mathrm{~m}$ for $\tau$ $=60 \mathrm{~min}, \phi_{m}=0.6$ and at the impeller speeds of: a) $50 \mathrm{rpm}$, and b) $500 \mathrm{rpm}$. 174 Figure 5.5-8 Contours of MMA monomer mass fraction at $\tau=45 \mathrm{~min}, \phi_{m}=0.7$, and impeller speeds of: a) $50 \mathrm{rpm}$, b) $100 \mathrm{rpm}$, c) $200 \mathrm{rpm}$, d) 300 , and e) $500 \mathrm{rpm}, \mathrm{T}=65^{\circ} \mathrm{C}$ 175 Figure 5.5-9 Contours of MMA monomer mass fraction at $\tau=60 \mathrm{~min}, \phi_{m}=0.7$, and impeller speeds of: a) $50 \mathrm{rpm}$, b) $100 \mathrm{rpm}$, c) $200 \mathrm{rpm}$, d) 300 , and e) $500 \mathrm{rpm}, \mathrm{T}=65^{\circ} \mathrm{C}$. 176 


\section{Chapter 1}

\section{INTRODUCTION}

Polymerization processes complex processes. There exists a wide variety of operating factors that can cause the production of polymer with totally different characteristics than the previous one. During polymerization, mixing and heat transfer have significant influence on the colloidal stability of latex particles. In emulsion polymerization, mixing, that causes in the bulk movement of the fluid, plays a vital role in maintaining the homogeneity of the reaction mass (Matyjaszewski and Davis, 2002, Patel el al., 2010a). The particle size distribution in emulsion is a key element in determining the mass and heat transfer rates through interfacial areas. Besides, it plays a crucial role in the design and scale-up of chemical reactors, mixers and separators. The size distribution influences the molecular weight distribution and polymer architecture, and consequently latex properties.

Complex systems require powerful computational tools to avoid expensive large-scale experiments and speed-up process and equipment optimization. Computational fluid dynamics (CFD) is a rapidly evolving discipline oriented on developing computational tools for solving problems related to transport processes (Paul et al., 2004; McKenna et al., 1998). CFD provides the numerical solution of the mass, momentum, and energy conservation equations. It also deals with the modeling of turbulence (Soon et al., 1999), multiphase flows (Bouaswaig et al., 2008), and reactive systems (Patel et al. 2010a and 2010b), which have more complex flow aspects (Eskin and Derksen, 2011). It is important to emphasize that an emulsion polymerization system incorporates complicated flow features such as multiphase mixture (Reyes and Asu, 2010; Stubbs and Sundberg, 2008) and turbulence (Farzi et al., 2011; Paquet and Ray, 1994). 
Even though significant advances have been achieved in emulsion polymerization in recent decades, the basic concept of mixing which is the basis for the formation of emulsion mixture has not been fully delineated yet. This is probably due to the conflicting results so far by various researchers (Nomura et al., 2005). Besides, estimation of the particle size distribution for an emulsion polymerization agitated in a mixing vessel has not been extensively investigated in both experimental and modeling aspects.

The objective of this work is to study the impact of mixing conditions on the emulsion polymerization. Therefore, a new laboratory scale reactor was set up to study the effect of operating conditions and reactor configurations such as impeller speed, impeller type, impeller number and baffles on the monomer conversion, the polymer molecular weight, particle size and size distributions, transition glass temperature, and number of particles. To be able to visualize some of the mixing aspects, a novel CFD model was developed based on the population balance and reaction kinetics for the emulsion polymerization. Since the modeling of emulsion polymerization in a stirred tank reactor is very complex, two separate CFD models were developed at the beginning. In the first CFD modeling, the size distribution was estimated for a simple water and oil emulsion in a batch mixing tank at an unsteady state condition and population balance was used to determine the size distribution. In the second stage of this study, a CFD model was developed for solution MMA polymerization performed at a steady-state condition and the reaction kinetic model was coupled with the CFD model.

Finally, the two mentioned CFD modeling procedures were expanded to develop a CFD model of the emulsion polymerization system in a batch reactor in at unsteady state condition. The CFD model of the emulsion polymerization incorporates the reaction kinetic modeling through population balance concepts and the transport model of a stirred medium. 
Chapter two is organized in two sections: the first part is devoted to a thorough literature review of emulsion, emulsion polymerization, mixing, CFD modeling and scale up. In the second part, the research objective is presented.

Chapter three describes in details the experimental setup, procedures, measurement methods and design of experiments.

Chapter four is organized in three sections: the first one is dedicated to the development of the CFD model for the emulsion polymerization system and provides governing equations and reaction kinetics. The two other sections of Chapter four are devoted to the development of the CFD models for the water and oil emulsion and also modeling of solution MMA polymerization system.

Chapter five provides a thorough discussion of the experimental and CFD results. This chapter is divided into five sections. Each section is organized in three parts; introduction, results and discussion, and concluding remarks.

Finally, Chapter six summaries the major conclusions of this study and provides recommendations for future work. 


\section{Chapter 2}

\section{LITERATURE REVIEW}

\subsection{An emulsion system}

An emulsion is a mixture of two or more immiscible liquids. One or more phases are dispersed as small droplets in the other continuous phase. The size of droplets has a significant impact on the stability and rheology of emulsion (Sjoblom, 2001). In fact, the droplet size distribution in emulsion is a key element in determining the mass and heat transfer rates through interfacial areas in chemical, petroleum, mining, food, and pharmaceutical applications. Besides, it plays a crucial role in the design and scale-up of chemical reactors, mixers and separators (Luo and Svendsen, 1996). Water-in-oil emulsions are predominantly found in petroleum industry (Aichele et al., 2009; Neumann and Paczynska-Lahme, 1988). A number of researchers have conducted the experimental studies on water-in-oil emulsions and investigated the effect of different parameters on the droplet size distribution (e.g. Boxall et al., 2010; Ohtake et al., 1987 and 1988).

\subsection{Emulsion polymerization}

Emulsion polymerization is an important industrial process for the production of latex paints, rubbers, coatings and adhesives (Dimitratos et al., 1994). Acrylic resins, which have an important commercial application in paint industries, are prepared through the polymerization of acrylic and methacrylic acid or their corresponding esters. The key attribute of acrylic coatings is their resistance to hydrolysis during extended exterior exposure (weathering), hardness, gloss 
and high alkali and oxidation resistance. One of the most important processes to manufacture acrylic resin is emulsion polymerization (Seda Tigli and Evren, 2005).

Novel automotive underbody coatings (plastisols) can be prepared based on core-shell lattices produced from emulsion polymerization (Wang et al. 2004). Yet, MMA based plastisols have some drawbacks such as poor storage stability and more investigation is necessary to improve the characteristics of the product.

Emulsion polymerization, which is a heterogeneous free radical polymerization process, involves emulsification of the relatively hydrophobic monomer in water by an oil-in-water surfactant, followed by the initiation reaction with either a water-soluble initiator such as sodium per sulfate or an oil soluble initiator such as 2.2'-azobisisobutyronitrile (Bovey et al., 1965; Chern, 2006; and Gilbert, 1995). Mechanisms and kinetics are different from bulk (Ma et al., 2010; and Demir et al., 2007) or solution free radical polymerization (Maschio and Moutier, 1989; Beuermann et al., 1995; and Fleury et al., 1992). According to Hansen and Ugelstad (1978), the classical theory of Smith-Ewart for nucleation of particles in emulsion polymerization has for some time been disputed. In accordance with this theory the nucleation of particles takes place solely in the monomer swollen micelles which are transformed into polymer particles by absorption of radicals from the aqueous phase. In the case of more water-soluble monomers, the Smith-Ewart expression does not satisfactorily describe the particle nucleation. Thus, the homogenous nucleation mechanism for the formation of particle nuclei in the continuous aqueous phase was introduced (Chern, 2006). In this type of nucleation, radicals are initiated by the thermal decomposition of waterborne initiator, and they can grow in size via the propagation reaction with those monomer molecules dissolved in the continuous phase. The oligomeric radicals then become water insoluble when the critical chain length is reached. The hydrophobic oligomeric 
radical may thus coil up and form a particle nucleus in the continuous aqueous phase. This is followed by formation of stable primary particles via the limited flocculation of the relatively unstable particle nuclei to reduce the total oil-water interfacial area and adsorption of surfactant molecules on their particle surfaces to increase the particle charge density.

The principle behind the homogenous nucleation mechanism is that the rate of generation of particle nucleus is primarily governed by the initiator and reaction temperature. The amount of surfactant molecules and sulphate end-groups of oligomeric radicals available for stabilizing particle nuclei produced in the continuous aqueous phase control the extent of flocculation of particle nuclei. To determine which particle nucleation mechanism predominates in a particular emulsion polymerization system is not straightforward. Hansen and Ugelstad (1979a, 1979b, 1979c) suggested that both micellar nucleation and homogenous nucleation were operative in emulsion polymerization with a concentration of surfactant greater that its critical micelle concentration (CMC). Therefore, in this study, it is assumed both nucleation type occur in justifying the results as the surfactant concentration is above the CMC.

Generally, there are some important issues as the polymerization proceeds. At the beginning of the polymerization, emulsification and nucleation govern the course of the process. The monomer droplets have to be small enough to provide a negligible resistance to monomer transport from the monomer droplets to the growing particles. Thus, the actual rate of polymerization is mainly due to the intrinsic reaction rates involved and the transport of monomer from the monomer droplets to the growing particles (Ohmura et al., 2005). It should be noted that insufficient emulsification affects the nucleation stage and hence the course of the polymerization rate. The colloidal stability is a key issue during the stage of the particle growth. If colloidal stability is not achieved, coagulation or break up will occur, which may result in off- 
spec products and troublesome operation. Rheology, flow, and heat transfer become more important in the later stages of emulsion polymerization.

\subsection{Mixing and polymerization}

During polymerization, mixing and heat transfer have significant influence on the colloidal stability of latex particles. In emulsion polymerization, mixing, which results in the bulk movement of the fluid, plays a vital role in maintaining the homogeneity of the reaction mass. The importance of mixing in polymerization has been the subject of a few studies (Matyjaszewski and Davis, 2002, Patel el., 2010a and 2010b, Fathi Roudsari et al., 2013). In an emulsion polymerization system, mixing can play a significant role in the kinetics of the chemical reaction. In the beginning of the emulsion polymerization, reaction is controlled by diffusion mechanism; i.e. the monomer is supplied by diffusion to the growing submicron polymer particles. In case of very low agitation rates, larger droplets are generated and phase separation may occur which will limit diffusion mechanism (Fathi Roudsari et al., 2012). In the stirred dispersion, deformation of the droplets occurs as a result of shear force in the turbulent flow field. The droplets experience viscous shear stress, pressure variation along their surface, and turbulent velocity fluctuation (Fathi Roudsari et al, 2012). Break up occurs if the hydrodynamic force exceeds the stabilizing force originating from the interfacial tension and drop viscosity (Baldyga and Podgórska, 1998). The deformation and break up is characterized by the Weber number, which is proportional to the ratio of the inertia forces to the surface tension force. For vinyl acetate emulsion polymerization in a CSTR, the monomer conversion and the particle size were increased with an increase in the impeller speed (Ohmura et al., 2005). A vigorous agitation can result in reduced nucleation of particles. Type of impeller has been investigated on the emulsion polymerization (Fathi Roudsari et al., 2014a, Ozdeger et al., 1998, 
Nomura et al., 1972, Kemmere et al., 1998, Hong, 2008). Some researchers (Nomura et al., 1972) reported an optimum range of stirring speed over which the polymerization rate was not affected by the speed. Nevertheless, it was reported (Hong, 2008) that the number of particles decreased by using a larger impeller diameter and faster speed. This was attributed to the shear stress and its effect on the nucleation mechanism, and the aggregation of the unstable nuclei.

The increase in conversion by enhancing the mixing speed in the Taylor-Couette polymerization reactor was also reported. The mass transfer between the vortices was poor at low rotational speed (Liu et al., 2013). Generally, emulsification of the monomers has a critical effect in the formation of particles in emulsion polymerization (Matyjaszewski and Davis, 2002). This is due to the fact that insufficient emulsification leads to a broad particle size distribution, which also affects the other stages of the emulsion polymerization reaction (Kemmere et al, 1999) and ultimately decreases the product quality. For the particles formed in batch polymerization, the maximum size of the particles is achieved at the end of the growth stage, where monomer droplets cease to exist and polymer particles become continuously richer in polymer as polymerization proceeds (Sajjadi, 2007). Basically, the factors that can reduce the colloidal stability of latex are broadly divided into physical and chemical types. The destabilization due to the physical factors occurs through an increase in the average kinetic energy and the collision frequency of the particles, whereas the destabilization due to the chemical factors happens through the reduction of the inter-particle potential energy barrier. In particular, the particle aggregation by agitation occurs when particles collide with the sufficient transporting force, which is caused by the high velocity gradients in the fluid, to surmount the repulsive barriers due to the adsorbed surfactant. The prediction of the rate of the inter-particle collisions in the fluid was first reported by Von Smoluchowski (Oldshue, 1989) for the orthokinetic flocculation. This 
type of the flocculation mechanism is based on the generation of the velocity gradients within the wastewater to promote the particle interaction. In this case, a mild mechanical agitation promotes the rate of shear-induced aggregation. However, any further increase in the agitation speed or power does not appreciably enhance the solids distribution in the fluid.

In spite of the influence of mixing on emulsion polymerization kinetics, it has not been largely debated in the open literature. However, in some reported cases, conflicting results were obtained by different researchers on the effect of stirring on the rate of polymerization reaction (Nomura et al., 2005). It has been reported (Ozdeger et al., 1998) that at low solid content, changing the impeller type from the A310 hydrofoil (an axial-flow impeller) to the Rushton turbine (a radialflow impeller) did not affect the polymerization rate. However, at high solid content, using the A310 hydrofoil resulted in a slower polymerization rate with a more distinct second population of particles compared to the runs that the Rushton impeller was employed. Some researchers (Nomura et al., 1972) reported an optimum range of stirring speed over which the polymerization rate was not affected by the speed.

A study (Kemmere et al., 1998) on the colloidal stability of polystyrene and polyvinyl acetate latex systems showed that a large turbine impeller provided the highest heat transfer coefficient rate as compared to a pitched-blade impeller under the same conditions and no dependency of the coagulation behavior on process conditions in terms of energy dissipation, reactor scale, impeller type, and impeller diameter was observed. According to literature (Hong, 2008), the slope of coagulum formation versus impeller speed was higher for the Rushton turbine rather than the hydrofoil impeller. It was reported that the hydrofoil impeller, with a lower shear, produced a more stable reaction mixture. In another study, researchers (Choi and Lee, 2010) investigated the emulsion polymerization of vinyl acetate, ethylene and N-methylol acrylamide 
using the Rushton and hydrofoil impellers. According to these researchers, the emulsions synthesized using the Rushton impeller had a larger particle size than those prepared using the hydrofoil impeller due to the higher shear generated with the Rushton turbine. It was shown that (Cheng et al., 2012) the axial-flow impellers were more energy efficient for the gas-liquid-liquid macro-mixing than the radial-flow impellers.

\subsection{Latex product characteristics}

Some of the important characteristics of the latex products are particle size, molecular weight and their relative distributions, chemical composition distribution, and flow properties. The choice of the recipe, reactor configuration, and the process conditions strongly determine the quality of the latex product. The ability to control the emulsion polymerization process is essential to guarantee constant product properties (Congalidis and Richards, 1998). The main focus of most researchers in this field for the last few decades has been the incorporation of particular reactive agents during the course of polymerization to adjust the polymer properties.

Latex particle size is an important factor in emulsion polymerization. Some studies (Fontenot and Schork, 1993, Yu et al., 1995) indicated that the overall rate of the monomer conversion decreased as the particle size got larger for both macro and mini emulsions. The formation of larger particle sizes at low conversion was also reported (Parker et al., 1996) for MMA emulsion polymerization in a batch reactor with a redox initiator system. It was claimed that the particles may have become increasingly swollen by the monomer or by the occurrence of an eventual coalescence prior to the sample analysis (Parker et al., 1996). However, in the aforementioned studies, little information has been provided regarding the detailed mixing characterization in emulsion polymerization. In general, important characteristics of the latex products are particle size, molecular weight, and their relative distributions, chemical composition distribution, and of 
course the fragility properties. The choice of the recipe, reactor configuration, and the process conditions strongly determine the quality of the latex product. The ability to control the emulsion polymerization process is essential to guarantee constant product properties (Congalidis and Richards, 1998). In particular, many other studies focused on polymer chemistry and incorporation of chemical additives (Parker et al., 1996, Congalidis and Richards, 1998, Sood, 2004).

The particle size distribution in emulsion is a key element in determining the mass and heat transfer rates through interfacial areas. Besides, it plays a crucial role in the design and scale-up of chemical reactors, mixers and separators (Fathi Roudsari et al., 2012). In large-scale reactors, it is difficult to reproduce uniform mixing similar to that in small reactors, and this is a common source of variability in particle nucleation and hence in particle size distribution. The size distribution affects the radical distribution, which in turn influences the molecular weight distribution and polymer architecture, and consequently latex properties.

The molecular weight and the molecular weight distribution of emulsion polymer exhibit a significant influence on the mechanical and application properties of the polymer (Chern, 2006). The molecular weight of polymer in the latex is related to the growth of the polymer radicals which depends on the propagation rate constant, the concentration of the monomer in the particles, and the average number of the free radicals per particle.

\subsection{Nucleation and growth and emulsion polymerization}

Emulsion polymerization is a rather complex process because nucleation, growth and stabilization of polymer particles are controlled by the chemical kinetics mechanisms in combination with various colloidal phenomena (Chern, 2006). Although the nucleation period is quite short, generation of particle nuclei during the early stage of the polymerization plays a 
crucial role in determining the final latex particle size, particle size distribution, and the quality of latex products. Mixing has a significant effect on the reaction kinetics of the emulsion polymerization. In case of very low agitation rates, larger droplets are generated and the phase separation may occur which will limit the diffusion of monomer to the growing particles (Kemmere et al., 1999). In contrast, vigorous agitation can result in reduced nucleation of particles. Some studies have focused on the modeling of the nucleation and growth phenomena for emulsion polymerization systems. Penlidis et al. (1986), Semino and Ray (1995), and Sgard et al. (1998) modeled nucleation and growth and showed the conversion results. Bouaswaig et al. (2008) used a 2D model to obtain the size distribution of the particles formed during emulsion polymerization in a tubular reactor by incorporating the nucleation and growth processes in the population balance equation. Vale and McKenna (2005) reviewed modeling of particle size distribution in emulsion polymerization, where no significant effect of mixing was given in their report.

\subsection{CSTR and polymerization}

Continuous stirred-tank reactors (CSTR) are ubiquitously used for mixing and reaction systems. In particular, polymerization in CSTR reactors has been a challenge (Choi, 1986). Furthermore, in a polymerization system, the polymer reactor behavior becomes quite complex due to inherent non-linearity arising from polymerization kinetics and a rapid increase of viscosity.

\subsubsection{Mixing in CSTR}

Mixing, reactor temperature, inlet monomer, solvent and initiator concentrations are among factors that can have profound effects on polymerization rate in a CSTR (Adebekun et al., 1989, Choi, 1986, Rudin and Choi, 2012). Thus, mixing in a polymer reactor vessel is obviously not 
ideal. In a CSTR, the control of heat is alleviated due to more efficient removal of reaction heat with the effluent and these reactors are economically attractive for industrial production with relatively infrequent changes in product properties (Rudin and Choi, 2012). Besides, a polymerization system in a CSTR is less influenced by the formation of thick polymer layers at the walls. However, only detailed simulations of the flow pattern and the kinetics for a given reactor with a specific geometry allows identifying the optimal reactor type.

\subsubsection{Effect of reactants flow rate on CSTR}

In addition to the mixing factor, there exist other important operating parameters in continuous reactors. The inlet monomer and initiator flow rate can have a profound effect on the polymerization rate as discussed elsewhere (Schmidt and Ray, 1981, Maschio and Moutier, 1989).

\subsection{Effect of solvent and gel effect on solution polymerization}

The effect of solvent has been investigated on solution polymerization. For instance, it has been reported that the rate of polymerization depends markedly on the solvent chemical structure (Gupta and Mandal, 1990); in contrast, it was noted elsewhere that chemically different solvents did not affect rate of polymerization (Beuermann et al, 1995). However, solvent can have a strong influence on the termination kinetics, which depends significantly on the conversion by the starting solvent fraction as reported (Beuermann et al, 1995).

Besides, the gel effect plays an important role in affecting of polymerization process at high conversions. It is nonlinearly dependent on solvent concentration and the operating temperature (Louie et al, 1985). In general, the gel effect phenomenon has been observed to greatly influence the average molar mass values and in turn, lead to more complicated reactor behavior. 
Description of the gel effect can be found in literature according to different theories (Louie et al., 1985, Fleury et al., 1992). Indeed, as polymerization proceeds, the reaction medium becomes more increasingly viscous, thus impeding the diffusion of growing polymer molecules due to growing chain entanglements (Louie et al., 1985, Ghosh et al., 1998, Tulig and Tirrell, 1982). Consequently, termination of the growing polymer chains does not occur as frequently as the propagation. This results in a net increase in the observed rate of polymerization or the so called auto-acceleration that can be related to reaching critical value in the free volume (Schmidt and Ray, 1981). For higher conversions, the termination rate reduces exponentially due to diffusion of polymer radicals (Schmidt and Ray, 1981, Verros and Achilias, 2009). Schmidt and Ray's kinetic modeling based on the fractional free volume theory (Schmidt and Ray, 1981, VivaldoLima, 1994) was reported to be applicable over the whole range of conversion (Adebekun et al., 1989, Maschio and Moutier, 1989, Ghosh et al., 1998, Ponnuswamy and Penlidis, 1988).

\subsection{Methyl methacrylate polymerization}

In case of a highly exothermic reaction, such as methyl methacrylate (MMA) polymerization, dead zones and hot spots may appear in some regions of the reactor vessel, thus leading to nonhomogeneous mixing reaction medium and eventually affecting the product quality (Adebekun et al. 1989, Choi, 1986). Some researchers have developed kinetic models of MMA solution polymerization in stirred tank reactors (Adebekun et al., 1989, Choi, 1986; Schmidt and Ray, 1981, Terrazas-Moreno et al., 2008). For instance, Adebekun et al. (1989) investigated the effect of the reactor operating conditions on the molar mass distribution of solution MMA polymerization, whereas Choi (1986) analyzed the steady-state operation of a solution polymerization in a CSTR. Schmidt and Ray (1981) developed a mathematical model along with the kinetic parameters for solution polymerization of MMA in a CSTR. In most studies (Choi, 
1986; Terrazas-Moreno et al., 2008) perfect mixing was assumed to derive mass and energy balance equations of MMA solution polymerization. Kim and Laurence (1998) studied the effects of feed and coolant temperature, residence time, and solvent volume fraction in an imperfectly mixed MMA polymerization. They considered a small mixing zone within the vicinity of the impeller and a large zone for the rest of reactor vessel.

\subsection{Modeling development}

\subsubsection{Population balance technique}

Population balance approach is generally employed to model the size distribution of the droplets, bubbles or crystals, which evolve and change with the flow due to phenomena such as nucleation, growth, coalescence, and breakage. The population balance model is a balance equation of species of different sizes and it is similar to the mass, energy and momentum balances, to track the changes in the size distribution. Early, Randolph and Larson (1971) proposed a solution for the population balance equation in a well-mixed batch system. They used the moment transform to convert the population balance equations into ordinary differential equations. Later, Hounslow et al. (1988) employed the moment transform to develop a numerical technique to model the growth and aggregation of particles in a suspension (calcium oxalate monohydrate crystals) and simulated the formation of kidney stones. Their approach incorporated the calculation of the rate of change of total particle number and volume. Over the last few decades, several methods have been proposed to solve the population balance equation in a dispersion: the standard method of moments (SMM) (Randolph and Larson, 1971), the Monte Carlo method (Ramkrishna, 2000), the Direct Quadrature Method of Moments (DQMOM) (Fox, 2009, Marchisio et al., 2003, Marsall et al., 2011), and the Discrete method 
(Hounslow et al., 1988, Kumar and Ramkrishna, 1996, Litster et al., 1995, Ramkrishna, 2000). Direct Quadrature method of moments is very efficient for single component systems and it is currently a well known and successful option to interface the population balance equation coupled with fluid dynamics. In a single component system within the scope of population balance modeling, only one property of particle is determined by population balance equation which can be mass, moisture content, volume, etc. (Qamar and Warnecke, 2007). However, extension to a two-component system is not straightforward and needs to be assessed against known solutions. Of a particular interest is the discrete method, in which the population balance is fully discretized into size intervals and the size distribution that is coupled with fluid dynamics is computed. This approach is also particularly useful when the range of particle sizes is known. Although, the discrete method is computationally expensive if the required number of intervals is large. Coulaloglou and Tavlarides (1977) theoretically investigated droplet breakage and coalescence in an emulsion (water in kerosene-dichlorobenzene) under turbulent flow regime in an agitated tank. They reported that the droplets coalescence rate was proportional to the droplets collision rate and the coalescence efficiency of the deformable drops. Hill and $\mathrm{Ng}$ (1995) developed a systematic method based on geometric ratios to use discretized population balance equations in the simulation of breakage processes in a batch system. Luo and Svendsen (1996) developed a theoretical model in order to predict breakup rates in turbulent liquid-liquid and gasliquid dispersions. Each particle was assumed to break up into only two smaller particles (binary breakage) and the frequency of breakage was considered to be proportional to the energy level of the arriving eddies towards the particles. A few theoretical breakage mechanisms were proposed by Kostoglou et al. (1997) for dispersion systems using different geometrical ratios for particles; however, considerable simplification was made to eliminate the breakage frequency. Patruno et 
al. (2009) reproduced experimental droplet data with different breakage kernels and compared them to those reported by Coulaloglou and Tavlarides (1977), Martínez-Bazán et al. (1999), and Sathyagal et al. (1996).

\subsubsection{Computational fluid dynamics}

Nowadays, the advanced computational fluid dynamics (CFD) techniques are utilized to simulate the multiphase flow processes. Some researchers have tried to couple the population balance equation with transport equations through the CFD modeling of the slurries, emulsions, and gasliquid systems; however, few of those models were actually employed to calculate the droplet/particle size distribution. For instance, Heath and Koh (2003) coupled CFD with the population balance to model the aggregation of solid particles in a slurry system. They used the Eulerian approach and $\mathrm{k}-\varepsilon$ model to simulate the turbulent two-phase flow. To reach convergence, the population balance equations were coupled with CFD in the last phase of computation. The size distribution of solid particles was not considered and only the variation of the mean particle size with time was taken into account. Agterof et al. (2003) employed CFD modeling to estimate the droplet size in oil in water emulsion. They calculated only mean drop size evolution with time and size distribution was not obtained. They employed the moments of droplet size for the population balance. Another drawback was that their simulation was based on a sequential approach. Kerdouss et al. (2008) employed discrete method for population balance modeling and used Eulerian-Eulerian approach for multiphase flow combined with k- $\varepsilon$ turbulent model for a gas-liquid (air in water) dispersion in a tank using MRF technique. Luo's model was used for breakage and coalescence; however, only the contours of mass transfer coefficient and Sauter mean diameter were presented and no results were presented regarding the bubble size distribution. Schütz et al. (2009) combined transport equations with turbulence model and 
population balance and used Lehr's approach (Lehr, 2001) to model the breakage and coalescence for the liquid-liquid (water-diesel) separation in the hydrocyclones. They employed mixture model for their multiphase model. CFD coupled with DQMOM population balance was employed by Silva and Lage (2011), Silva et al. (2010), and Silva et al. (2008) to model the aggregation and breakage process in a 1-D domain. They mainly reported the calculation of the computation times in serial and parallel modes (Silva and Lage, 2011), comparison of some quadrature-based methods (Silva et al., 2010) and comparison of different CFD packages (Silva et al., 2008). However, in all three papers, they provided only some contours and graphs of Sauter mean diameter and the size distribution was not calculated. It must be mentioned that their simulations were not validated by any experimental data. Also, the population balance equations were not solved simultaneously and sequential solution method was utilized in these three studies. Besides, they implemented the laminar regime while a turbulent flow is generally required for the emulsion systems. Srilatha et al. (2010) considered the droplets breakage and coalescence and used the discretized population balance model to estimate the drop size distribution for two different emulsion systems (water-in-tri butyl phosphate and water-inxylene) in a stirred tank. The CFD technique was employed to calculate the size distribution in different zones of the mixing tank. However, they assumed that the coalescence and breakage occurred only between two droplets with equal size in a steady state mode. Besides, they could not combine CFD and population balance due to high computational requirements and performed their modeling in sequential approach. Selma et al. (2010) modeled a multiphase flow in a gasliquid column and stirred tank reactor using the Eulerian-Eulerian approach with a standard k- $\varepsilon$ model for turbulence. The problem was solved by coupling the population balance equation with CFD utilizing the direct quadrature method of moments (DQMOM) and discrete method and 
only the Sauter mean diameter was calculated considering the coalescence and breakage phenomena. In a very recent paper by Beyraktar et al. (2011), the discrete method was applied for solving the population balance equations. However, they employed sequential solution methods, i.e. the turbulent flow field was firstly simulated and then the population balance equations were solved. In addition, due to the extensive computational time for the calculation of the hydrodynamic variables coupled with population balance equations, they recommended parallelization of model for future works to reduce the computational costs. Vladisavljevic et al. (2011) studied the effect of oil phase viscosity and the frequency of formation of droplets in an oil-in-water microchannel emulsification system and obtained drop size distribution in their CFD modeling; however, no information was given regarding their CFD simulation. As mentioned above, quite a number of studies have focused on the modeling of the droplet breakage and coalescence phenomena, which play a significant role in the design of the dispersion processes. Nevertheless, the prediction of the particle sizes in an inhomogeneous flow, where the flow velocity is changing spatially in magnitude and direction and with time, is not yet well recognized. The essence is that the break-up and coalescence processes determine source terms in a population balance equation while the velocity vectors obtained from the transport equations determine the convective term.

CFD has provided a new vision in a number of polymerization processes as well. Serra et al. (2007) investigated CFD modeling of styrene free radical polymerization in a T-junction micro reactor and assumed isothermal conditions in a micro-scale reactor to calculate poly-dispersity index, degree of polymerization and conversion. CFD was also estimated to calculate convection heat transfer coefficient between particles and polymer fluids in an ethylene polymerization fluidized bed reactor (Dehnavi et al. 2008). A study on CFD analysis of polymerization claimed 
that the gradients of temperature and concentration were only necessary for poorly designed agitation systems and perfect mixing was a good assumption (Poubel et al., 2010). However, this claim has provoked controversy. A few researchers incorporated the chemical kinetics into the CFD model (Kolhapure and Fox, 1999, Patel et al., 2010a and 2010b), however the population balance was not employed to obtain the particle size distribution.Kolhapure and Fox (1999) studied small-scale mixing of reactant species inside a tubular low-density polyethylene (LDPE) reactor. Their CFD results proved that imperfect mixing reduced monomer conversion and increased the poly-dispersity index. Patel et al. (2010a and 2010b) investigated the CFD modeling of styrene bulk polymerization in a CSTR and studied the effects of the impeller speed, the input-output stream locations, and residence time on the polymerization conversion. On the other hand, some researchers (Maggioris et al., 2000) considered the population balance in their CFD modeling, however they did not incorporate the chemical reaction kinetics into the CFD model. Maggioris et al. (2000) reported reduction in polymer drop size in suspension polymerization when the stirring rate was increased in their CFD simulations. Vivaldo-Lima et al. (1998) calculated the particle size distribution using the population balance technique and a compartment-mixing model in a mixing tank. They included the beak-up and coalescence (but no kinetics) for suspension polymerization and used CFD to estimate the turbulence dissipation rate. Alexopoulos et al. (2002) employed a two-bladed pitched turbine for a suspension polymerization system at turbulent regime in a 2D domain and studied the effect of the turbulent kinetic energy and dissipation rate on the evolution of the droplet size using the $k$ - $\varepsilon$ turbulence model. However, the polymerization kinetics was not employed in their model.

In a study conducted by Pohn et al. (2013), a CFD model was coupled to a multi-zonal population balance to scale up a semi-batch styrene emulsion polymerization reactor. The effect 
of mixing was investigated on the latex particle size distribution. Some researchers (Pohn et al., 2010, Pohn et al., 2011) employed a pitched bladed turbine in a general latex system. They considered sequential manner for solving equations and employed a laminar regime in a 3D domain. However, their model was not validated and the reaction kinetics was not considered. Elgebrandt et al. (2005) studied the effect of viscosity on the conversion in a semi-batch reactor using a pitched blade impeller in a 2D domain. However, the employed shear rate in their model was lower than their experiments and the model was not validated. Besides, the kinetics was not incorporated into their model. They showed the coagulation rate coefficient for two agitation rates under turbulent regime. In another study, Elgebrandt et al. (2006) coupled a kinetic model with multi compartment zone 2D CFD model and solved equations in a sequential method. Do Amaral et al. (2004) modeled the mini emulsion polymerization and used CFD to show flow patterns as input for modeling the droplet size distribution, but the reaction kinetics was not considered.

\subsubsection{Sequential or simultaneous solution}

One of the important computational issues is the solution method of the transport equations coupled with the turbulence and population balance equations, which can be sequential or simultaneous solution. In sequential technique, first the velocity flow field and the turbulence model parameters are calculated and then population balance equations are solved. On the other hand, in simultaneous solution approach, all the equations are solved from the beginning of the simulation at the same time. The accuracy of the obtained results is definitely influenced by solution method (Fathi Roudsari et al., 2012). 


\subsubsection{Parallelization}

Another significant issue is using parallelization in computation. In order to calculate the particle size distribution, rather than obtaining only mean size diameter, parallelization in computation is recommended in literature to reduce extensive computational time and cost (Fathi Roudsari et al., 2012 and 2013, Patel et al. 2010a and 2010b).

\subsection{Scale up}

The impact of stirring becomes more pronounced as the reactor size increases and the creation of the effective mixing throughout the reactor becomes a hard task. Therefore, an appropriate criterion should be considered for the scale-up of the mixing system (Oldshue, 1989). Different scale-up criteria such as constant power/volume and constant tip velocity have been proposed in the literature while the geometric similarity exists between the small-scale and large-scale mechanically agitated reactors (Okufi et al., 1990; and El-Hamouz et al. 2009). However, these scale-up criteria are not compatible with each other. For instance, at constant power/volume, the agitation speed and shear rate are altered considerably (Afshar Ghotil et al., 2013; Holland and Bragg, 1995; and Paul et al., 2004). For liquid-liquid dispersions, Baldyga et al. (2001) showed that the scale-up resulted in an increase in Reynolds number, larger turbulent fluctuations, and smaller drops. Some researchers believed that the scale-up criterion based on the same mixing time is not reliable and proposed two dimensionless groups as a function of the Reynolds number for a variety of impeller-vessel configurations (Nere et al., 2003).

Besides, a few studies regarding the scale up of continuous reactors have been reported. For instance, Campolo et al. (2002) used Nagata's correlation (Nagata, 1975) for the scale up of a CSTR from a lab scale to an industrial scale and verified the scale up of the two reactors by comparing the power number, discharge flow number, and pumping efficiency. In another study, 
the reaction time, heat removal capacity, and the degree of homogeneity were kept constant for the solution and bulk polymerization. Therefore, need of high micro-mixing efficiency and heat transfer on one hand and the scale-up possibilities on the other hand should be considered (Meyer, 2003). In one study it was suggested that two different types of mixers have to be employed to fulfill the above-mentioned requirements (Meyer, 2003). Furthermore, some correlations are available for a CSTR scale up using the geometric ratio, Froude number, and pumping capacity (Fogler, 2006). In addition, according to Fogler (2006), the information regarding the rate of reaction as a function of conversion may be sufficient to scale up a lab-scale reactor to an industrial-scale reactor. However, there is very little knowledge about the rate of reaction as a function of conversion (Fogler, 2006). For the emulsion polymerization scale-up, the goal is to produce commercial scale latexes of the same quality as those developed in the laboratory. Because of geometric considerations, the larger the volume of the reactor, the smaller is its heat transfer area/volume ratio. Therefore, larger reactors require longer process times to carry out the process under good thermal control. Although agitation may improve the heat transfer, the range of the mixing intensity is limited because a vigorous agitation may cause shear induced coagulation. In large-scale reactors, it is difficult to reproduce uniform mixing similar to that in small reactors, and this is a common source of variability in particle nucleation and hence in particle size distribution. The size distribution affects the radical distribution, which in turn influences the molecular weight distribution and polymer architecture, and consequently latex properties. Besides, most researches are performed using jacket cooling on a lab-scale reactor. However, for large reactors, jacket cooling is not always sufficient to obtain reasonably short batch times and supplemental heat removal through external heat exchangers, internal cooling coils, and baffles must be used (Asua, 2007). 


\subsection{Research objective}

The thorough literature review conducted revealed that little effort has been devoted to the impact of mixing on emulsion polymerization systems. Thus, the main objective of this study is to investigate the impact of the most important mixing parameters (e.g. impeller speed, impeller type, impeller number, and baffles) on the monomer conversion, polymer molecular weight, particle size and size distributions, transition glass temperature, and number of particles. To achieve this objective, an experimental setup was designed to conduct emulsion polymerization, collect experimental data, and measure the latex characteristics. To visualize the complex reactive flow inside the polymerization reactor, a novel CFD model was developed based on the population balance and reaction kinetics by incorporating the nucleation and growth phenomena while taking into account the velocity gradients generated by the impeller rotation inside the emulsion polymerization reactor. 


\section{Chapter 3}

\section{EXPERIMENTAL SET UP AND PROCEDURE}

This chapter is divided in a few sections including experimental set-up, procedure, measurement methods, experimental design and error analysis.

\subsection{Experimental set-up}

The experimental set-up used in this study consisted of a reactor vessel, the impellers (pitched blade and Rushton), baffles, U shaped cooling coil, heating jacket and a circulator.

Figure 3.1-1 shows the schematic diagram of the experimental set up which is comprised of a $2 \mathrm{~L}$ PARR reactor vessel (series 4530) connected to a temperature controller heating/cooling bath and also temperature control unit (series 4848). The controller can be auto-tuned for the desired set point temperatures. The reactor is a flat bottomed cylindrical tank with a diameter of 10.16 $\mathrm{cm}$ and a height of $26.67 \mathrm{~cm}$ with a total capacity of two litres. Four equally spaced baffles with the height of $0.19 \mathrm{~m}$ and width of $\mathrm{T} / 10$ were mounted in the reactor as well. The baffles were attached to reactor wall as the internal space was limited. 


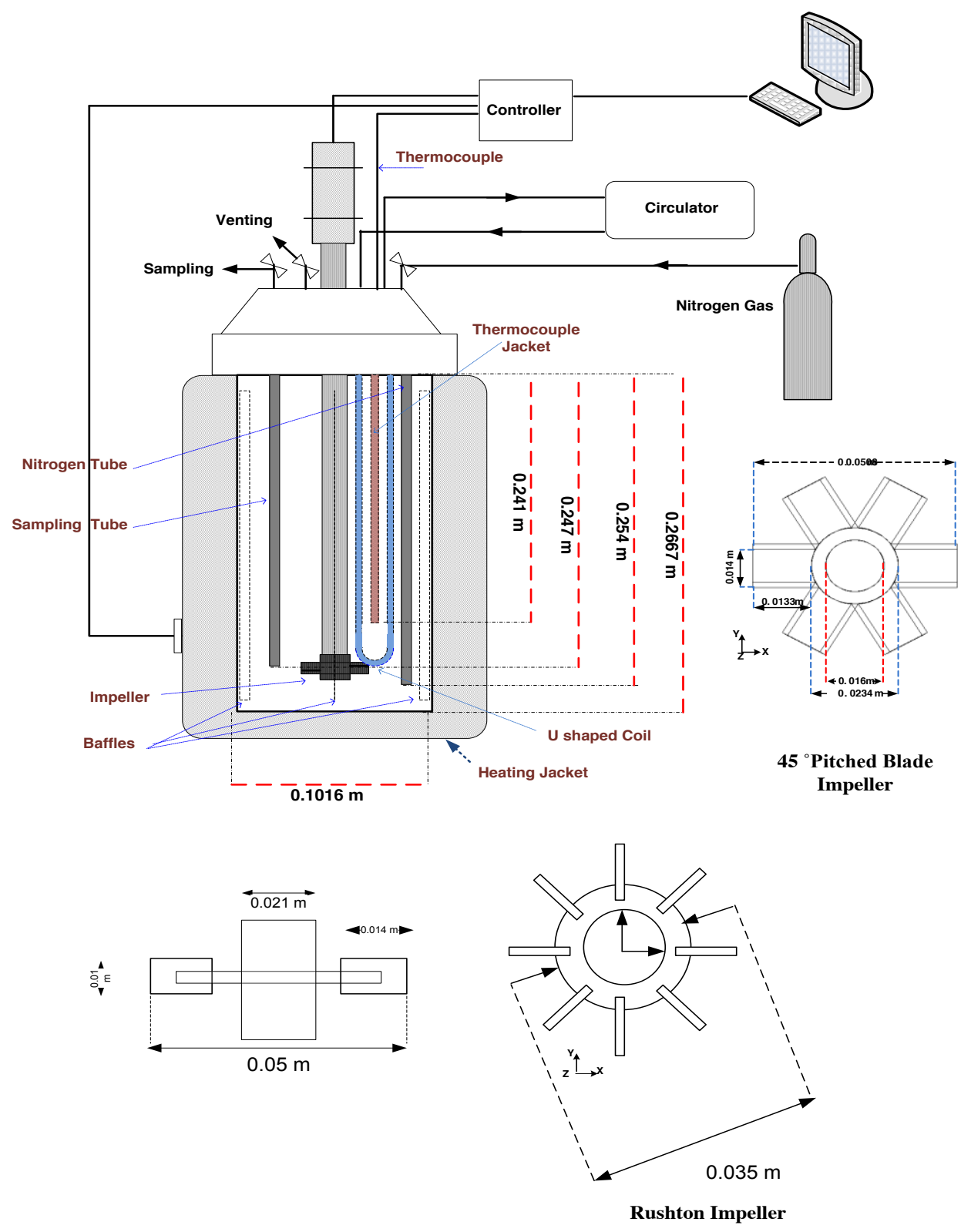

Figure 3.1-1 Schematic diagram of the experimental set up.

The reactor vessel was equipped with one or two impellers of the following types: $45^{\circ}$ pitchedblade turbine with a diameter of $5.0 \mathrm{~cm}$, or Rushton impeller with a diameter of $5.0 \mathrm{~cm}$. The offbottom clearance was $1.3 \mathrm{~cm}$ and in case of using two impellers, the inner distance was $8.0 \mathrm{~cm}$ between the two impellers. The pitched-blade turbine is an axial-flow impeller, which generates 
flow along the impeller axis. The axial-flow impellers are designed to generate high bulk flow with low turbulence. The Rushton impeller is a radial-flow impeller, which discharges flow along the impeller radius. The radial-flow impellers are designed to produce high shear and turbulence within the mixing vessels (Paul et al., 2004). Thus, in this study, we selected these two types of impellers to explore the effect of flow type and pattern on the emulsion polymerization.

A U-shaped cooling coil connected to the circulator bath, a thermocouple jacket, and inlet and outlet pipes for sampling and gas purging were attached to the reactor. The power of motor is $1 / 4$ hp. A cooling bath (Cole-Parmer Polystat H28L) was connected to the cooling coil.

\subsection{Experimental procedure}

The experimental procedure involves the reaction reagents and the reaction mixture preparation.

\subsubsection{Reaction reagents}

The following reagents were used in the polymerization reaction: methyl methacrylate (MMA) with purity of $99 \%$ as the monomer, sodium dodecyl sulphate (SDS) with purity over $99 \%$ as the surfactant, deionized (DI) water, potassium persulphate (KPS) as the initiator, and hydroquinone with 99\% purity as the inhibitor. All reagents were supplied by Sigma Aldrich Canada and used as received. Emulsions were prepared according to the following recipe: $700 \mathrm{~g}$ water, $250 \mathrm{~g}$ methyl methacrylate, and $8.64 \mathrm{~g}$ of sodium dodecyl sulphate (SDS). The amount of potassium persulphate (KPS) was $0.5455 \mathrm{~g}$ or $2.9 \times 10^{-3} \mathrm{~mol} / \mathrm{L}$ water that is typical for an emulsion polymerization system (Guo et al., 1997; Penlidis, 1986). The volumetric ratio of monomer to water was $4 / 10$. 


\subsubsection{Mixture preparation}

The desired amount of SDS was dissolved in $80 \mathrm{~g}$ of water while stirred with a magnetic stirrer bar. Amount of $600 \mathrm{~g}$ of water and the SDS aqueous solution were added to the reactor. $250 \mathrm{~g}$ of MMA were then poured into the reactor. The reactor temperature was set to $50^{\circ} \mathrm{C}$ or $60^{\circ} \mathrm{C}$ and was sealed and purged with nitrogen gas for $20 \mathrm{~min}$.

The venting valve was opened and closed several times in order to remove the air completely from the reactor. The impeller speed was varied from 20 to $350 \pm 2 \mathrm{rpm}$. The potassium persulphate (i.e. initiator) was then dissolved in $20 \mathrm{~g}$ of water and injected into the reactor with a syringe at $45^{\circ} \mathrm{C}$ for the reactor set point of $50^{\circ} \mathrm{C}$ and at $55^{\circ} \mathrm{C}$ for the cases when reactor set point was $60^{\circ} \mathrm{C}$.

Samples $(20 \mathrm{~mL})$ were at first taken $5 \mathrm{~min}$ after adding the initiator. The sampling procedure continued at an interval time of $10-15 \mathrm{~min}$ for a total reaction time of $130 \mathrm{~min}$. Each sample was poured into a vial and 2 drops of $1 \%$ hydroquinone solution were immediately added to stop the reaction. The vials were placed in tray of ice for 10 to 15 minutes and then put in the refrigerator.

\subsection{Measurement methods}

\subsubsection{Determination of conversion}

In the present study, off-line gravimetry was employed to determine the latex sample conversion levels. An aliquot of each sample in the vials (1-2 ml) was transferred into a dry and clean aluminum cup and was weighed. The content of the cup was then dried in a vacuum oven (24 hours at $40^{\circ} \mathrm{C}$ ) and the cup with the dry residue was weighed again. The weight fraction of solids is then given by (Penlidis, 1986): 


$$
w . f . \text { solids }=\frac{(\text { weight of cup }+ \text { dry residue })-(\text { weight of cup })}{(\text { weight of cup }+ \text { wet sample })-(\text { weight of cup })}
$$

The weight fraction of initiator and emulsifier is known from the employed experimental recipe. Therefore, the conversion is (Penlidis, 1986):

$$
x(t)=\frac{(w . f . \text { solids }-w . f . \text { initiator }-w . f . \text { mulsifier })}{w \cdot f . \text { monomer }}
$$

\subsubsection{Particle size determination}

The particle size and particle size distribution can significantly impact the quality and applications of the latex product. Both variables were determined by light scattering method using Microtrac Particle Analyzer (Model S3000/S3500).

The advanced S3500 Tri-Laser uses a detector system located at a precise distance from the point where the particles interact with the light. The detectors also respond to the intensity of light reaching them, which is related to the amount present of a particular particle size.

\subsubsection{Molar mass measurements}

The measurements of the polymer molecular weights were performed using gel permeation chromatography (GPC) (Viscotek, Model 302-040) equipped with a triple detector array. Tetrahydrofuran (THF) was used as the mobile phase at a nominal rate of $1.0 \mathrm{~mL} / \mathrm{min}$. The filtered dry polymers were dissolved in THF (1-5 mg polymer/mL THF) and the molecular weight averages were obtained using universal calibration of PMMA standards. 


\subsubsection{Glass transition temperature determination}

Differential Scanning Calorimeter (DSC) (Model Q2000 V24.10 Build 122) was utilized to measure the glass temperature of polymer samples under nitrogen atmosphere $(50.0 \mathrm{~mL} / \mathrm{min})$. The scan rate was $10^{\circ} \mathrm{C} / \mathrm{min}$ over the temperature range $40-190^{\circ} \mathrm{C}$. The $T_{g}$ values for the dried polymer samples were determined from the second scan of the DSC curve.

\subsubsection{Scanning electron microscopy (SEM) micrographs}

In order to illustrate the size and size distribution of PMMA latex particles, the scanning of electron microscopy (SEM) images was performed by Hitachi S-2150. The latex dispersion was diluted and dropwise deposited onto a glass surface. Once dried the sample was sputter coated with a thin $\mathrm{Pt} / \mathrm{Pd}$ film. 


\subsection{Experimental design}

Minitab-16 was employed for statistical design of experiments and data analysis. After the preliminary tests, the selected ranges for the impeller speed, impeller type, number of impellers, baffles and temperature are given in Table 3.4-1. The experimental designs are illustrated in Table 3.4-2 and Table 3.4-3. The reaction temperature of $60^{\circ} \mathrm{C}$ has been employed by some researchers (Tanrisever et al., 1996; Ming et al., 1998). Furthermore, we selected a lower temperature (i.e. $50^{\circ} \mathrm{C}$ ) to examine the mixing effects at this condition. The conversion normally increases at the higher temperature as it has already been reported in the literature (Bao et al., 2004). In contrast, at a lower reaction temperature the particle size distribution is more uniform. In our experiments, we intended to investigate whether improving the mixing condition can enhance the polymer characteristics at lower temperatures (i.e. $\left.50^{\circ} \mathrm{C}\right)$. The design of experiment consisted of 16 runs for the first part and 12 runs for the second part. Recipe was constant for all experiments listed in Table 3.4-2 and Table 3.4-3. Some of the runs were repeated three times.

The experiments were replicated three times for the Rushton impeller at the rotational speeds of 100, 250 and $350 \mathrm{rpm}$, and for the pitched impeller at the rotational speed of $250 \mathrm{rpm}$. Since the conversion profiles had the same trend for the replicated experiments, we concluded that repeating all the tests was not necessary since the chemical recipe and the temperature were the same for all the experiments. 
Table 3.4-1 Experimental ranges and level of factors.

\begin{tabular}{|lcl|}
\hline Factor & Levels & Values \\
\hline Speed & 3 & $100,250,350$ \\
Impeller Type & 2 & Rushton, Pitched blade \\
Number of Impellers & 2 & 1,2 \\
Baffles & 2 & Yes, No \\
Temperature & 2 & $50^{\circ} \mathrm{C}, 60^{\circ} \mathrm{C}$ \\
\hline
\end{tabular}


Table 3.4-2 Experimental design 1 of the experiments.

\begin{tabular}{|cccc|}
\hline No $^{1}$ & Reaction Temperature $\left({ }^{\circ} \mathbf{C}\right)$ & Impeller Speed (rpm) & Baffles \\
\hline 1 & 50 & 20 & Yes \\
2 & 50 & 20 & No \\
3 & 50 & 250 & Yes \\
4 & 50 & 250 & No \\
5 & 50 & 100 & Yes \\
6 & 50 & 100 & No \\
7 & 50 & 350 & Yes \\
8 & 50 & 350 & No \\
9 & 60 & 20 & Yes \\
10 & 60 & 20 & No \\
11 & 60 & 250 & Yes \\
12 & 60 & 250 & No \\
13 & 60 & 100 & Yes \\
14 & 60 & 100 & No \\
15 & 60 & 350 & Yes \\
16 & 650 & & \\
\hline
\end{tabular}

\footnotetext{
${ }^{1}$ Experiments 7, 11, 12, 14 and 16 were repeated 3 times.
} 
Table 3.4-3 Experimental design 2 of the experiments

\begin{tabular}{|cccc|}
\hline No $^{1}$ & Impeller type & Impeller speed (rpm) & Number of impellers \\
\hline 1 & Rushton & 250 & Single \\
3 & Pitched & 250 & Single \\
4 & Pitched & 100 & Double \\
5 & Rushton & 100 & Single \\
6 & Pitched & 100 & Single \\
7 & Pitched & 350 & Single \\
8 & Pitched & 250 & Double \\
9 & Rushton & 100 & Double \\
10 & Pitched & 350 & Double \\
11 & Rushton & 350 & Double \\
12 & Rushton & 350 & Single \\
\hline
\end{tabular}

\footnotetext{
${ }^{1}$ Experiments 1, 2, 4, and 11 were repeated 3 times.
} 


\subsection{Error analysis}

The error regarding the conversion measurement can be defined using the standard deviation (SD). The error bars are shown in conversion profiles as described in Chapter 5, Figure 5.1-1 and Figure 5.2-1. Error bar $(X, \bar{Y}, E)$ function plots mean value $\bar{Y}$ versus $\mathrm{X}$ with symmetric error bars $2 \times \mathrm{E}$ long. $\mathrm{E}^{1}$ is the standard deviation of measurements.

${ }^{1} E=\sqrt{\frac{1}{N} \sum_{i=1}^{N}\left(Y_{i}-\bar{Y}\right)^{2}}$ where $N$ is the number of measurements, and $Y$ is the variable and $\bar{Y}$ is the mean value of the measurements. 


\section{Chapter 4}

\section{CFD MODEL DEVELOPMENT}

This chapter is divided into three sections. The CFD modeling of MMA emulsion polymerization is provided in the first section. In this part of this chapter, the transport equations are coupled with the reaction kinetics through the population balance with incorporation of user defined functions of nucleation and growth rates for a batch reactor in an unsteady state mode. Since the techniques employed for the CFD modeling of the emulsion polymerization is complex, two preliminary CFD modeling have been performed which are discussed in the second and third section of this chapter. The second section is devoted to the CFD modeling of a simple water and oil emulsion agitated in a mixing tank. The focus of this part was to incorporate the population balance equation into the CFD model developed for the mixing of two immiscible liquids. The third section is dedicated to the development of a CFD model for MMA polymerization in a CSTR. In this part, the reaction kinetics were incorporated into the CFD model through user defined functions. 


\subsection{Modeling of the emulsion polymerization system}

\subsubsection{Reactor system}

The reactor system has been fully described in Chapter 3 and the schematic diagram is shown is Figure 3.1-1.

\subsubsection{Mathematical model}

ANSYS Design Modeler 14.5 was utilized to discretize the 3D computational domain via unstructured tetrahedral cells (Figure 4.3-1). To capture the flow details near the flow domain boundaries, a size function was employed to generate very refined mesh in the vicinity of the rotating impeller and the reactor walls. To test for grid independence, the mesh was fine-tuned based on the velocity, turbulence, and polymer volume fraction profiles computed near the impeller, where is the region of large velocity gradients. Grid independence was achieved when the additional cells did not change the calculated velocity, turbulence, and polymer volume fraction near the impeller blades by more than $5 \%$. The final three-dimensional model had approximately 320,000 cells. Several researchers have used MRF (multiple reference frames) method for the similar mixing geometry and achieved good results (Fathi Roudsari et al., 2012, Patel et al. 2010a and 2010b, Kerdouss et al., 2008). In this study, we employed MRF technique to capture the motion of the rotating impeller in a stationary tank. The transport equations inside and outside of the rotating frame were solved separately, and as the numerical solution proceeded a transfer of information was considered at the interface. 

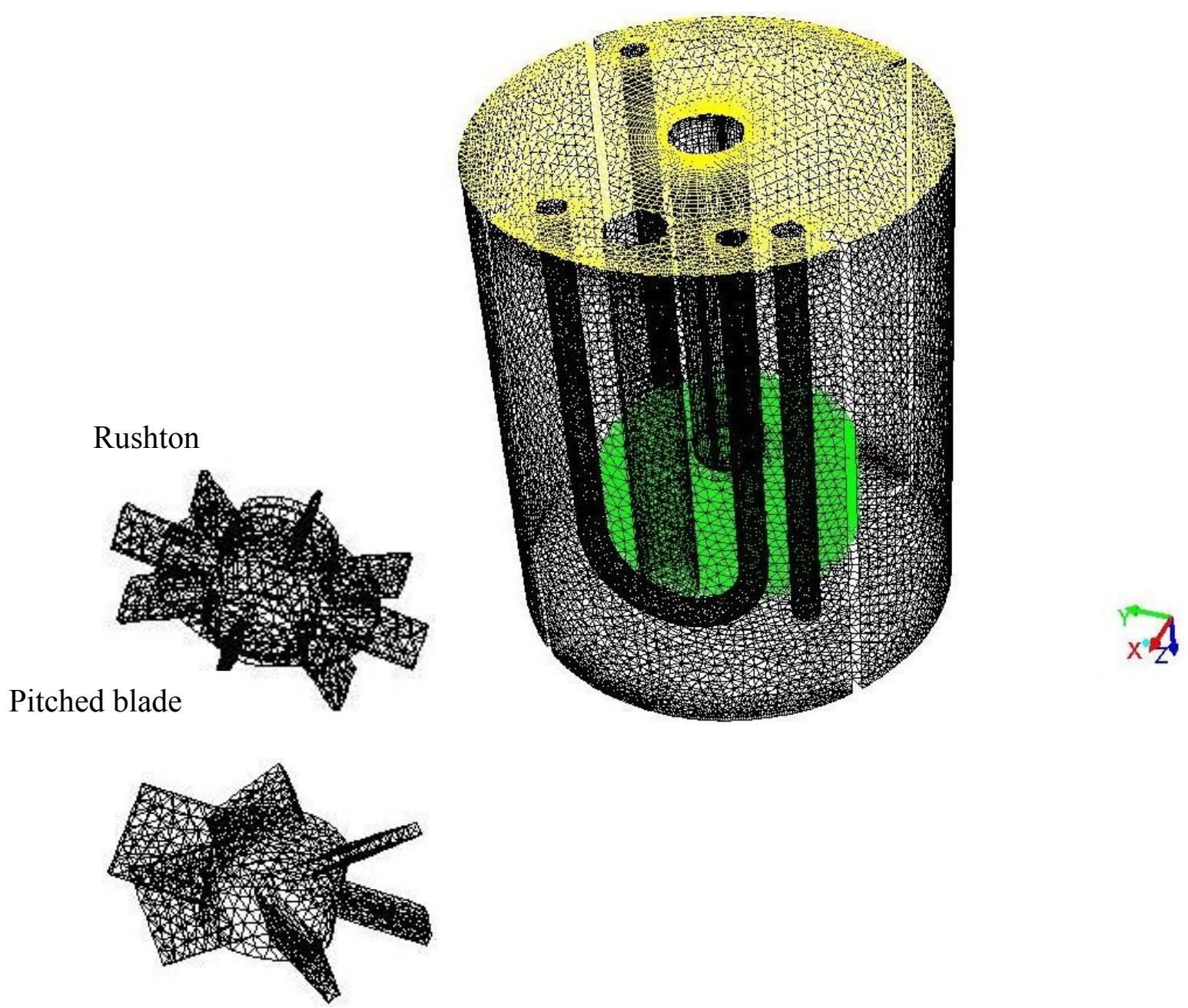

Figure 4.1-1 Geometry grid. 
The fluid height was $0.119 \mathrm{~m}$. The reactor was equipped with a pitched-blade turbine or a Rushton impeller with a diameter of $5.00 \mathrm{~cm}$ and four baffles spaced at $90^{\circ}$ intervals against the wall. The impeller diameter and off-bottom clearance from the tank were $0.050 \mathrm{~m}$ and $0.013 \mathrm{~m}$, respectively. The impeller speed was varied from 20 - $350 \mathrm{rpm}$. For the CFD modeling, the multiphase flow was considered inside the reactor vessel in which the first phase had two species, i.e. methyl methacrylate monomer and water, while the secondary phase consisted of polymer methyl methacrlyate particles. Mixture model approach was used as a multiphase model and a set of continuity and momentum equations was considered for each phase and species. Since the Reynolds number was in the range of 300-5000 when the impeller speed was varied from 20 to $350 \mathrm{rpm}$, the turbulent flow was assumed in the simulations.

Assuming a turbulent and transient condition, the governing transport equations are presented here (Versteeg and Malalasekera, 2007).

The general continuity equation for the mixture is (Ranade, 2002):

$$
\begin{gathered}
\left(\frac{\partial}{\partial t}\left(\rho_{m i x}\right)\right)+\nabla \cdot\left(\rho_{m i x} \vec{v}_{m i x}\right)=\sum_{p=1}^{L}\left(\dot{m}_{p q}-\dot{m}_{q p}\right)+\bar{S}_{q} \\
\vec{v}_{\text {mix }}=\frac{\sum_{q} \alpha_{q} \rho_{q} \vec{v}_{q}}{\rho_{\text {mix }}} \\
\rho_{\text {mix }}=\sum_{q} \alpha_{q} \rho_{q}
\end{gathered}
$$


where $\vec{v}_{\text {mix }}$ is the mixture velocity and $\rho_{\text {mix }}$ is the mixture density, $\alpha_{q}$ is the phase volume fraction, and is the velocity of phase q. $\dot{\boldsymbol{m}}_{p q}$ and $\dot{\boldsymbol{m}}_{q p}$ are mass transfer from phase $\mathrm{p}$ to phase $\mathrm{q}$ ( shows mass transfer from phase $\mathrm{q}$ to $\mathrm{p}$ ) and $\bar{S}_{q}$ denotes the source mass generated in phase $\mathrm{q}$. In our CFD modeling, the continuity equation for the second phase can be written as follows (Ranade, 2002):

$$
\left(\frac{\partial}{\partial t}\left(\rho_{m i x}\right)\right)+\nabla \cdot\left(\rho_{m i x} \vec{v}_{m i x}\right)=\dot{m}
$$

The $\dot{m}$ is defined as the mass transfer from the primary phase to the secondary phase.

The general momentum equation for phase $\mathrm{q}$ is expressed as (Ranade, 2002):

$$
\begin{aligned}
& \frac{\partial}{\partial t}\left(\rho_{m i x} \vec{v}_{m i x}\right)+\nabla \cdot\left(\vec{v}_{m i x} \vec{v}_{m i x}\right)=\nabla P+\nabla \cdot\left[\mu_{m i x}\left(\nabla \vec{v}_{m i x}+\nabla \vec{v}_{m i x}{ }^{T}\right)\right] \\
& +\rho_{m i x} \vec{g}+\nabla \cdot\left(\sum_{q} \alpha_{q} \rho_{q} \vec{v}_{d r, q} \vec{v}_{d r, q}\right)+ \\
& \sum_{p=1}^{n}\left(K_{p q}\left(\vec{v}_{p}-\vec{v}_{q}\right)+\dot{m}_{p q} \vec{v}_{p q}-\dot{m}_{q p} \vec{v}_{q p}\right)+\left(\vec{F}_{q}+\vec{F}_{l i f t, q}+\vec{F}_{v m, q}\right)
\end{aligned}
$$

Here $\vec{g}$ is the acceleration due to gravity, $\mathrm{P}$ is the pressure, $\vec{F}_{q}$ is the external force, $\vec{F}_{l i f t, q}$ is a lift force, $\vec{F}_{v m, q}$ is a virtual mass force. $\vec{v}_{p q}$ is the interphase velocity if the phase $p$ mass is being transferred to phase $q$. Likewise, $\vec{v}_{q p}$ is used if the phase $q$ mass is being transferred to phase $p$. $K_{p q}$ is the interphase momentum exchange coefficient. $\mu_{m i x}$ is the viscosity of the mixture and $\vec{v}_{d r, q}$ is the drift velocity for secondary phase q:

$$
\mu_{\text {mix }}=\sum_{q=1} \alpha_{q} \mu_{q}
$$




$$
\vec{v}_{d r, q}=\vec{v}_{q}-\vec{v}_{\text {mix }}
$$

The lift force is insignificant compared to the drag force in emulsions since the particle diameters are much smaller than the inter-phase spacing and therefore this term can be neglected (Drew and Lahey, 1993). The virtual mass effect is significant when the secondary phase density is much smaller than the primary phase density. In this study, the density of the secondary phase (i.e. polymer) was larger than the density of primary phase (i.e. water and monomer mixture) and therefore, the virtual mass force was not included (Drew and Lahey, 1993). In our CFD modeling the momentum equation for the mixture is expressed as follows (Ranade, 2002):

$$
\begin{aligned}
& \frac{\partial}{\partial t}\left(\rho_{\text {mix }} \vec{v}_{\text {mix }}\right)+\nabla \cdot\left(\vec{v}_{\text {mix }} \vec{v}_{\text {mix }}\right)=\nabla P+\nabla \cdot\left[\mu _ { \text { mix } } \left(\nabla \vec{v}_{\text {mix }}+\nabla_{\left.\left.\vec{v}_{m i x}{ }^{T}\right)\right]}\right.\right. \\
& +\rho_{\text {mix }} \vec{g}+\nabla \cdot\left(\sum_{q} \alpha_{q} \rho_{q} \vec{v}_{d r, q} \vec{v}_{d r, q}\right)+K_{p q}\left(\vec{v}_{p}-\vec{v}_{q}\right)+\dot{m} \vec{v}_{p}
\end{aligned}
$$

The inter-phase momentum exchange coefficient (Ranade, 2002) is defined as:

$$
K_{p q}=\frac{\alpha_{q} \alpha_{p} l}{\tau_{q}}
$$

in which $\tau_{a}$, the particulate relaxation time, is as follows:

$$
\tau_{q}=\frac{\rho_{q} D_{q}{ }^{2}}{18 \mu_{p}}
$$

where $D_{q}$ is the diameter particle in phase $q$. Equation (8) was used to calculate the particulate relaxation time. The drag function $(l)$ was calculated according to Schiller and Naumann model (Ishii and Zuber, 1979; Schiller and Naumann, 1933). The drag expression proposed by Schiller and Naumann (Ishii and Zuber, 1979) can be employed for the solid spherical particles or for the fluid particles that are sufficiently small and may be considered spherical such as our case in this 
study. For non-spherical particles, the user should supply the drag curve from experiment (Ranade, 2002; Schiller and Naumann, 1933). Furthermore, according to Loth (2008), empirical Schiller-Naumann expression is appropriate as long as the particle deformation is negligible. A few researchers have employed this drag expression and achieved good results (Schütz et al., 2009; Silva et al., 2008; Yarin and Hetsroni, 1994). Therefore, in this study, the drag function $(l)$ is calculated according to Schiller and Naumann model (Ishii and Zuber, 1979; Schiller and Naumann, 1933):

$$
l=\frac{C_{D} \operatorname{Re}}{24}
$$

where

$$
\begin{aligned}
& C_{D}=24\left(1+0.15 \mathrm{Re}^{0.687}\right) / \operatorname{Re} \quad \operatorname{Re} \leq 1000 \\
& C_{D}=\mathbf{0 . 4 4} \quad \operatorname{Re}>\mathbf{1 ~ O O O}
\end{aligned}
$$

$R e$ is the relative Reynolds number and is defined for continuous phase $q$ and the dispersed phase $p$ as follows:

$$
\operatorname{Re}=\frac{\rho_{p}\left|\vec{v}_{q}-\vec{v}_{p}\right| d_{q}}{\mu_{p}}
$$

in which $\mu_{\mu_{p}}$ is the viscosity of the continuous phase.

The species continuity equation for the multiphase mixture can be represented as follow (Ranade, 2002):

$$
\begin{aligned}
& \frac{\partial}{\partial t}\left(\alpha_{q} \rho_{q} W_{q i}\right)+\nabla \cdot\left(\alpha_{q} \rho_{q} \vec{v}_{q} W_{q i}\right)=-\nabla \cdot \alpha_{q} J_{q i}+\alpha_{q} \bar{S}_{q i}+ \\
& \sum_{p=1}^{n}\left(\dot{m}_{p^{i} q^{j}}-\dot{m}_{q^{j} p^{i}}\right)+R_{q i}
\end{aligned}
$$


$W_{q i}, R_{q i}, J_{q i}$ are the local mass fraction of each species for phase $q$, the rate of production of species $i$ by chemical reaction for phase $q$ and the mass diffusion in the turbulent flow respectively. $\bar{S}_{q i}$ is the rate of mass production by user-defined sources.

The species continuity equation for the multiphase mixture can be represented as follow (Ranade, 2002):

$$
\begin{aligned}
& \frac{\partial}{\partial t}\left(\alpha_{q} \rho_{q} W_{q i}\right)+\nabla \cdot\left(\alpha_{q} \rho_{q} \vec{v}_{q} W_{q i}\right)=-\nabla \cdot \alpha_{q} \vec{J}_{q i}+\alpha_{q} S_{q i}+ \\
& \sum_{p=1}^{n}\left(\dot{m}_{p^{i} q^{j}}-\dot{m}_{q^{j} p^{i}}\right)+R_{q i}
\end{aligned}
$$

$W_{q i}, R_{q i}, J_{q i}$ are the local mass fraction of each species for phase $q$, the rate of production of species $i$ by chemical reaction for phase $q$ and the diffusive flux in the turbulent flow respectively. $S_{q i}$ is the rate of mass production by user-defined sources.

In our CFD modeling, the species continuity equation for the second phase can be written as follows:

$$
\frac{\partial}{\partial t}\left(\alpha_{q} \rho_{q} W_{q i}\right)+\nabla \cdot\left(\alpha_{q} \rho_{q} \vec{v}_{q} W_{q i}\right)=-\nabla \cdot \alpha_{q} \vec{J}_{q i}+\dot{m}
$$

As previously mentioned, $\dot{m}$ is defined as the mass transfer from the primary phase to the secondary phase by population balance mechanism.

The diffusive flux in the turbulent flow is computed by:

$$
\vec{J}_{q i}=-\left(\rho_{m i x} D I F_{i}+\frac{\mu_{t u r b}}{S c_{t}}\right) \nabla W_{q i}
$$

$D I F_{i}$ is the mass diffusivity for specie $i$ which was $1.9 \times 10^{-9} \mathrm{~m}^{2} / \mathrm{s}$ for methyl methacrylate monomer in water (Kemmere and Meyer, 2005). Sc $c_{t}$ is the turbulent Schmidt number $\frac{\mu_{\text {turb }}}{\rho_{q}(D I F)_{\text {turb }}}$ 
where $\mu_{\text {turb }}$ is the turbulent viscosity and $D I F_{\text {turb }}$ is the turbulent diffusivity. The turbulent viscosity and diffusivity equations will be shortly defined in this section. The default $S c_{t}$ number is 0.7 as an empirical constant and is relatively insensitive to the molecular fluid properties. Hence, there was no need to alter the default value (Tominagaa and Stathopoulosb, 2007).

The $k-\varepsilon$ model is a robust and validated turbulence model that has been used extensively in industrial engineering confined flows where the shear stresses are more important (Ranade, 2002). The drawback is that it does not perform well in unconfined flows such as external aerodynamics applications. Reynolds stress model (RSM) (Raikar et al., 2009) includes several parameters such as stabilization of turbulence and can be employed for non-circular ducts and curved flows, on the other hand, RSM often demands a significant increase in computing time due to the additional equations and reduced convergence, and it has not been validated widely. In this study, the standard $k-\varepsilon$ model was used to model the turbulent flow within the emulsion cell and a good model agreement was achieved with the experimental data. The $k-\varepsilon$ model has already been utilized elsewhere (Raikar et al., 2009; Wang et al., 2005, Zucca et al., 2006). The turbulence kinetic energy, $k_{t u r b}$, and its rate of dissipation, $\varepsilon_{\text {turb }}$, are described as follows (Launder and Spalding, 1974; Hoffmann and Chiang, 2000):

$$
k_{t u r b}=\frac{1}{2}\left[\overline{\vec{v}_{x}^{\prime 2}}+\overline{\vec{v}_{y}^{\prime 2}}+\overline{\vec{v}_{z}^{\prime 2}}\right]
$$

and

$$
\varepsilon_{\text {turb }}=\frac{\mu_{t u r b}}{\rho_{\text {mix }}} \overline{\left(\frac{\partial \vec{v}_{x}^{\prime}}{\partial x_{y}}\right)\left(\frac{\partial \vec{v}_{x}^{\prime}}{\partial x_{y}}\right)}
$$


where $\vec{v}^{\prime}$ is fluctuation term for velocity (Bird et al., 2007) and the turbulent viscosity and diffusivity are calculated as follows:

$$
\begin{gathered}
\mu_{t u r b}=\rho_{m i x} C_{\mu} \frac{k_{\text {turb }}^{2}}{\varepsilon_{\text {turb }}} \\
D_{\text {turb }}=\frac{\partial}{\partial x_{z}}\left(\frac{\mu_{\text {turb }}}{\sigma_{k}} \frac{\partial \overrightarrow{\vec{v}}_{x}^{\prime} \vec{v}_{y}^{\prime}}{\partial x_{z}}\right)
\end{gathered}
$$

where $_{C_{\mu}}=0.09$ and $\sigma_{\sigma_{k}}=1.0$ (Ranade, 2002).

Finally, the values for $k_{t u r b}$ and $\varepsilon_{t u r b}$ in CFD model were calculated from the following transport equations:

$$
\frac{\partial}{\partial t}\left(\rho_{\text {mix }} k_{\text {turb }}\right)+\frac{\partial}{\partial x}\left(\rho_{\text {mix }} k_{t u r b} \vec{v}_{x}\right)=\frac{\partial}{\partial z}\left[\left(\mu+\frac{\mu_{t}}{\sigma_{k}}\right) \frac{\partial k_{\text {turb }}}{\partial z}\right]+G_{k}+G_{b}-\rho_{\text {mix }} \varepsilon_{\text {turb }}
$$

$$
\begin{aligned}
& \frac{\partial}{\partial t}\left(\rho_{\text {mix }} \varepsilon_{\text {turb }}\right)+\frac{\partial}{\partial x}\left(\rho_{\text {mix }} \varepsilon_{\text {turb }} \vec{v}_{x}\right)=\frac{\partial}{\partial z}\left[\left(\mu_{\text {mix }}+\frac{\mu_{\text {turb }}}{\sigma_{\varepsilon}}\right) \frac{\partial \varepsilon_{\text {turb }}}{\partial z}\right]+ \\
& C_{1 \varepsilon} \frac{\varepsilon_{\text {turb }}}{k_{\text {turb }}}\left(G_{k}+C_{3 \varepsilon} G_{b}\right)-C_{2 \varepsilon} \rho_{\text {mix }} \frac{\varepsilon^{2}}{k_{\text {turb }}}
\end{aligned}
$$

In these equations, $G_{b}$ is the generation of turbulence kinetic energy due to buoyancy. $G_{k}$ represents the generation of turbulence kinetic energy due to the mean velocity gradients:

$$
G_{k}=-\rho \overline{v_{i}^{\prime} v_{j}^{\prime}} \frac{\partial \vec{v}_{z}}{\partial x}
$$

and the model constants had the following default values (Ranade, 2002):

$C_{1 e}=1.44, C_{2 e}=1.92$ and $\sigma_{\sigma_{e}}=1.3$ 
which are derived experimentally for turbulent shear flows and decaying isotropic turbulence (Blazek, 2005). $C_{3 \varepsilon}$ is $\tanh \left|\frac{\vec{v}_{z}}{\vec{v}_{x}}\right|$ where $\vec{v}_{z}$ is the component of the flow velocity parallel to the gravitational vector and $\vec{v}_{x}$ is the component of the flow velocity perpendicular to the gravitational vector (Henkes et al., 1991).

The population balance equation was coupled with turbulent and multiphase flow equations to obtain the nucleation and growth rate of particles in the secondary phase. The population balance equation is written in terms of a set of discrete size classes or bins and the particles are discretized into $L$ sizes (Kerdouss et al., 2008; Hagesaether et al., 2002; Chen et al., 2005):

$$
\frac{\partial}{\partial t}\left(\rho_{s} \alpha_{k}\right)+\nabla \cdot\left(\rho_{s} \vec{v}_{k} \alpha_{k}\right)+\frac{\partial}{\partial V}\left(G_{v} \rho_{s} \alpha_{k}\right)=\rho_{s} V_{0} \dot{N}_{T}^{\prime}
$$

The volume fraction of polymer particle size $\mathrm{k}$ is defined as

$$
\alpha_{k}=N_{k}^{\prime} V_{k} \quad k=0,1, \ldots, L-1
$$

Considering $V_{k}$ the particle volume and $n_{n^{\prime}(V, t)}$ the number of particle in bin size $k$ per unit volume of reactor, then $N_{k}^{\prime}$ is the total number of particles per unit volume of reactor as follows:

$$
N_{k}^{\prime}(t)=\int_{V_{k}}^{V_{k+1}} n^{\prime}(V, t) d V
$$

The term $\rho_{s} V_{\mathrm{o}} \dot{N}^{\prime}$ in the population balance equation, only appears in the case of the smallest $(k=0)$ particle size $\mathrm{V}_{0} . \rho_{s}$ is the secondary phase density, i.e. polymer density. 
The total volume fraction of the secondary phase is defined as $\alpha_{s}$ and $\alpha_{k}$ is the volume fraction of polymer particles in the secondary phase with radius of $r_{k}$ :

$$
f_{k}=\frac{\alpha_{k}}{\alpha_{s}}
$$

The growth rate $G_{v}$ based on particle volume is discretized as follows (Hounslow et al., 1988):

$$
\frac{\partial\left(G_{v} \rho_{s} \alpha_{k}\right)}{\partial V}=\rho_{s} V_{k}\left[\left(\frac{G_{v, k-1} n_{k-1}^{\prime}}{V_{k}-V_{k-1}}\right)-\left(\frac{G_{v, k} n_{k}^{\prime}}{V_{k+1}-V_{k}}\right)\right]
$$

The mass transfer $\dot{m}$ from the primary phase to the secondary phase due to nucleation and growth rate is calculated by (Hosseini et al., 2012):

$$
\dot{m}=\rho_{s} V_{0} \dot{N}_{T}^{\prime}+\sum_{k} \rho_{s} G_{v, k} n_{k}^{\prime}
$$

The nucleation and growth rates were written in $\mathrm{C}$ code and linked to the CFD model as the user defined functions (UDF) as shown in Appendix A.1. 


\subsubsection{Reaction kinetics}

When a surfactant (emulsifier) is dissolved in water, several physical properties of the solution such as surface tension change. Beyond a particular level of concentration, there is a sudden change in the slope of solution properties. This concentration is called the critical micelle concentration (CMC) (Kumar and Gupta, 2003). Beyond the CMC, the surfactant molecules stay primarily in micellar form. The surfactant molecules aggregate and form micelles. The initiator decomposes to give a hydrophilic radical and start polymerization inside the monomer swollen micelles. Emulsion polymerization in batch generally comprises three stages. First stage, in which free radicals generated in the aqueous phase enter micelles and form polymer particles, second stage, where particles grow as they absorb monomer from monomer droplets, until their depletion, and third stage, where polymerization continues within the particles until the monomer is consumed. A three stage process also occurs in semibatch emulsion polymerization reactions: a batch period in which a small fraction of the total monomer amount is polymerized; an addition period in which the rest of the monomer is flowed into the reactor ("growth stage"); a post addition period to allow for polymerization of residual monomer. After the first stage, particle nucleation may be complete or, in some conditions, nucleation can continue during the feeding period (Sajjadi and Brooks, 1999).

Basic reactions of emulsion polymerization and the reaction rates (Harada et al., 1972; Rawling a nd Ray, 1988) are presented as follows:

$\begin{array}{lll}\text { Initiator decomposition } & I_{\text {in }} \stackrel{k_{d}}{\longrightarrow} 2 R_{\text {in }}^{\bullet} & r_{i n}=2 f k_{d}\left[I_{\text {in }}\right] \\ \text { Formation of particles } & \boldsymbol{R}_{\text {in }}^{\bullet}+m_{s} \stackrel{k_{1}}{\longrightarrow} N^{\bullet} & k_{1}\left[m_{s}\right]\left[R_{i n}^{\bullet}\right]\end{array}$ 
Initiation of particles

Propagation in particle

Termination

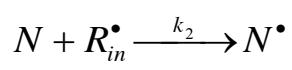

$$
\boldsymbol{R}^{-}{ }_{j}+M \stackrel{k_{p r o p}}{\longrightarrow} R^{-}{ }_{j+1}
$$

$$
N^{\bullet}+R^{\bullet} \stackrel{k_{2}}{\longrightarrow} N
$$

$$
k_{2}[N]\left[R_{i n}^{\bullet}\right]
$$

$k_{\text {prop }}[M]\left[N^{\bullet}\right]$

$k_{2}\left[N^{\bullet}\right]\left[R^{\bullet}\right]$

In the initial decomposition reaction, $\boldsymbol{I}_{i n}, \boldsymbol{R}_{i n}^{-}, \boldsymbol{r}_{i n}, \boldsymbol{k}_{\boldsymbol{d}}$ and $f$ present initiator concentration, initiator radical concentration in the water, generation rate of initiator radical, decomposition rate constant of initiator, and initiator efficiency, respectively.

In the formation of particles and termination reactions, $m_{s}, k_{1}, k_{2}$ and $N$ and $N^{\bullet}$ denote emulsifier micelle concentration, rate constant of formation of polymer particle, rate constant for initiation and termination of particles, number of dead polymer particles and number of active polymer particles, respectively.

In the propagation in particle and transfer to monomer reactions, $\boldsymbol{P}^{\cdot}{ }_{j}, k_{\text {prop }}$ and $M$ represent active polymer containing $j$ units, propagation rate constant and monomer concentration in polymer particle, respectively. The reaction rate constants have been listed in Table 4.1-1. According to Harada et al. (1972) the following equation can be written for the kinetic reactions:

$$
\frac{d R_{\text {in }}^{\bullet}}{d t}=r_{i n}-k_{1}\left[m_{s}\right]\left[R_{\text {in }}^{\bullet}\right]-k_{2} N_{T}\left[I_{\text {in }}^{\bullet}\right]
$$


The $r_{i n}$ is assumed to be constant since the half-life of the decomposition of potassium persulphate is sufficiently long compared to the reaction time. $N_{T}$ is the total number of particles. Applying the steady state method:

Table 4.1-1 Values of kinetic parameters for the emulsion polymerization chemical reaction rate constants.

\begin{tabular}{|ccc|}
\hline Parameter & Value & Reference \\
\hline$k_{d}$ & $1.8 \times 10^{17} \exp \left(-34100 /\left(\mathrm{Rg}_{\mathrm{g}} \mathrm{)}\right) \mathrm{s}^{-1}\right.$ & Rawlings and Ray \\
$f$ & 0.5 & Rawlings and Ray \\
$k_{\text {prop }}$ & $4.92 \times 10^{5} \exp \left(-4353 /\left(\mathrm{R}_{\mathrm{g} T} \mathrm{~T}\right) \mathrm{L} \mathrm{mol}^{-1} \mathrm{~s}^{-1}\right.$ & Rawlings and Ray \\
$k_{2}$ & $9.8 \times 10^{7} \exp \left(-701 /\left(\mathrm{R}_{\mathrm{g}} \mathrm{T}\right)\right) \mathrm{Lmol}^{-1} \mathrm{~s}^{-1}$ & Rawlings and Ray \\
$a_{s}$ & $35 \times 10^{-16} \mathrm{~cm}^{2}$ & Harada et al., 1972 \\
$\varepsilon=k_{2} N_{a g g r} / k_{1}$ & $1.28 \times 10^{5}$ & Harada et al., 1972 \\
& 0.5 & Harada et al., 1972 \\
\hline
\end{tabular}

$$
\begin{gathered}
\frac{d R_{\text {in }}^{\bullet}}{d t}=0 \\
R_{\text {in }}^{\bullet}=r_{i} /\left(k_{1}\left[m_{s}\right]+k_{2} N_{T}\right)
\end{gathered}
$$

The nucleation rate of polymer particle is: 


$$
\dot{N}_{T}=\frac{d N_{T}}{d t}=k_{1}\left[m_{s}\right]\left[R^{\bullet}\right]
$$

Substituting equation (4.1-33) into equation (4.1-34) yields:

$$
\dot{N}_{T}=\frac{d N_{T}}{d t}=\frac{r_{i n}}{1+k_{2} N_{T} / k_{1}\left[m_{s}\right]}=\frac{r_{i n}}{1+\varepsilon N_{T} / S}
$$

where $S$ is concentration of emulsifier and $\varepsilon$ is:

$$
\varepsilon=k_{2} N_{a g g r} / k_{1}
$$

And $N_{\text {aggr }}$ is the aggregation number of a micelle. The emulsifier concentration effective for micelle formation was $S$ and was calculated as follows (Harada et al., 1972):

$$
\begin{aligned}
& S=S_{0}-k_{v}\left(X M_{0}\right)^{2 / 3} N_{T}^{1 / 3} \\
& k_{v}=\left[\frac{36 \pi}{\left(1-\phi_{c}\right)^{2} a_{s}^{3} \rho_{p}^{2}}\right]^{1 / 3}
\end{aligned}
$$

where $X, M_{0}, \varphi_{c}, a_{s}$ and $\rho_{p}$ are monomer conversion, initial monomer concentration, critical monomer mass fraction in polymer particle, surface area occupied by an emulsifier molecule and density of polymer particles, respectively.

$$
S_{0}=S_{\text {in }}-S_{C M C}
$$

where $S_{i n}$ and $S_{C M C}$ are the concentration of emulsifier charged initially and critical micelle concentration of the emulsifier, respectively. The value of the critical micelle concentration was obtained from literature (Penlidis, 1986) as $4.7 \times 10^{18}$ molecules per $\mathrm{cm}^{3}$ water and $S_{\text {in }}$ and $S_{0}$ in 
Equation (4.1-38) were calculated as $2.57 \times 10^{19}$ and $2.1 \times 10^{19}$ molecules per $\mathrm{cm}^{3}$ water, respectively.

Harada et al. (1972) considered two limiting cases which can describe analytically the characteristic features of emulsion polymerization, especially nucleation of polymer particles. One case is where the initiation radicals generated in the aqueous phase enter into the micelles rather than into the polymer particles. In this case:

Case 1: $\quad \quad \& N_{T} / S<<1 \quad N_{T}=N^{\bullet}=r_{i} t$

Case 2 is the opposite of case 1, i.e.

Case 2: $\quad \varepsilon N_{T} / S>>1 \quad N^{\bullet}=N=\frac{N_{T}}{2}$

Our experimental data was in agreement with the case $2\left(N_{T}=2 r_{i} t\right)$.

The mass fraction of monomer in the polymer particles, $\varphi$ is nearly constant in the range below critical value and the variation of $\varphi$ with conversion $X$ is expressed by the following equation (Harada et al., 1972):

$$
\phi=1-X
$$

The number of polymer particles and the total volume of polymer particles remain almost constant above the critical value of $\varphi$. The critical value of $\varphi$ was considered to be $0.57(X=1-\varphi$ $=0.43)($ Harada et al., 1972).

It should be noted that the final nucleation rate linked to the population balance has the unit of particles formed per total volume per second (particles $\left./ m^{3} . s\right)$ :

$$
\dot{N}_{T}^{\prime}=7.29 \times 10^{5} \dot{N}_{T}
$$


In our CFD model, we utilized monomer mass fraction and the mixture density in the primary phase to calculate the monomer concentration as follows:

$$
\begin{aligned}
& C_{m t}=W_{m} \times \rho_{\text {mixture }} \\
& V=\frac{C_{m 0}-C_{m t}}{\rho_{s}} \times V_{\text {reactor }} \\
& r=(V \times 3 / 4 \pi)^{1 / 3}
\end{aligned}
$$

where $\rho_{s}$ and $V$ are the secondary phase (polymer) density and reactor volume, respectively. The conversion is defined by:

$$
X=\frac{C_{m 0}-C_{m t}}{C_{m 0}}
$$

The growth rate of the polymer particle based on particle radius is defined as (Hosseini et al., 2012; Rawlings and Ray, 1988):

$$
G_{r}=\frac{d r}{d t}=\frac{k_{\text {prop }}}{N_{A}} \times \frac{\bar{n}}{4 \pi r^{2}} \times \frac{\phi}{1-\phi}
$$

in which $N_{A}$ is the Avogadro number, $\bar{n}$ is the number of radicals per particle, and $r$ is the radius of particle.

In the user defined codes written for the CFD model, we first calculated the radius of particle and conversion based on equations (4.1-45) and (4.1-46). $\varphi$ was then obtained using equation (4.142) and finally the growth rate was calculated using equation (4.1-47).

The growth rate based on the particle volume, $G_{v}$ is defined as 


$$
\begin{aligned}
& G_{v}=\frac{\partial V}{\partial t}=3 K_{v}(2 r)^{2} G(r, t) \\
& K_{v}=\frac{\pi}{6}
\end{aligned}
$$

\subsubsection{CFD simulation}

ANSYS Fluent 14.5 was employed to solve all transport equations for the unsteady-state condition. All transport equations were integrated using control volume method. Standard wall function was considered as near wall treatment (Launder and Spalding, 1974). The distance between the first cell and the wall was bridged by wall functions (Blazek, 2005). On the liquid level, the symmetry boundary condition was used. First-order upwind discretization scheme was employed to calculate the face fluxes in the momentum and phase transport equations. The velocity-pressure coupling was solved using phase coupled SIMPLE algorithm (Patanakar and Spalding, 1972). Integrating the governing transport equations over small control volumes resulted in an algebraic linear system, which was solved with the Gauss-Seidel iterative method and the Algebraic Multi-Grid method (AMG). The reactor model was comprised of continuity equation, three momentum equations for the velocity components for the mixture of two phases, two $k$ and $\varepsilon$ turbulence equations, one species continuity equation for the monomer specie in the primary phase, seven equations for the bin fractions of the secondary phase and the continuity volume fraction equation for the secondary phase. The simulation started with all velocity components set to zero. The initial volume fraction of secondary phase was set to zero and the initial mass fraction of monomer in the primary phase was set to 0.26 . These conditions simulated the impeller-stirred tank to be initially at rest. The initial conditions for the turbulent energy dissipation and kinetic energy were assumed to be zero. The geometric ratio between 
seven bin sizes was equal to two and was chosen in such a way that the final mean particle size was located in the middle. The bin sizes at different impeller speeds are listed in Table 4.1-2.

Table 4.1-2 Diameter of the particle sizes used in the discrete method.

\begin{tabular}{|cccccccc|}
\hline CFD Bin & $\mathbf{0}$ & $\mathbf{1}$ & $\mathbf{2}$ & $\mathbf{3}$ & $\mathbf{4}$ & $\mathbf{5}$ & $\mathbf{6}$ \\
\hline Diameter Size & 1792 & 896 & 448 & 224 & 112 & 56 & 28 \\
$(\mathbf{n m})$ & & & & & & & \\
\hline
\end{tabular}

Initial volume fraction of all bins was set to zero. The population balance discrete model was enabled and nucleation and growth rate were set to zero at the start of simulation. All equations were simultaneously solved in an unsteady state mode with the step size of $0.1 \mathrm{~s}$, and maximum iteration per time step of 10. Then, the user defined functions as shown in Appendix A.1 for nucleation and growth rate were enabled and the equations were solved. Convergence was checked by monitoring the residuals of all mentioned equations, sum of velocity magnitude at the liquid surface, and monomer mass fraction. Supercomputing facilities of HPCVL (High Performance Computing Virtual Laboratory) was utilized to perform the computations. Each simulation was run in parallel with 36 dual cores SUN Ultra-Spark IV, 1.8GHz Sun MicroSystems CPUs. The grid was partitioned into 36 parts and one CPU was then assigned to each partition. The convergence for each unsteady state simulation was achieved after 3 days for 7 particle bin size fractions (classes) in the discrete model. 


\subsubsection{Convergence criteria}

The solution of the transport equations is an iterative process hence the field variables changes with the iteration till the convergence is reached. In this study, one of the most critical tasks in the CFD modeling was solution of transport equations of the species transport equation in the first phase species mass fractions on each node. Hence, monitoring the un-reacted methyl methacrylate monomer mass fraction in the first phase (water and monomer) against the iteration progress represented the convergence history of the species transport equations. Figure 4.1-2 shows the un-reacted monomer fraction in the first phase.

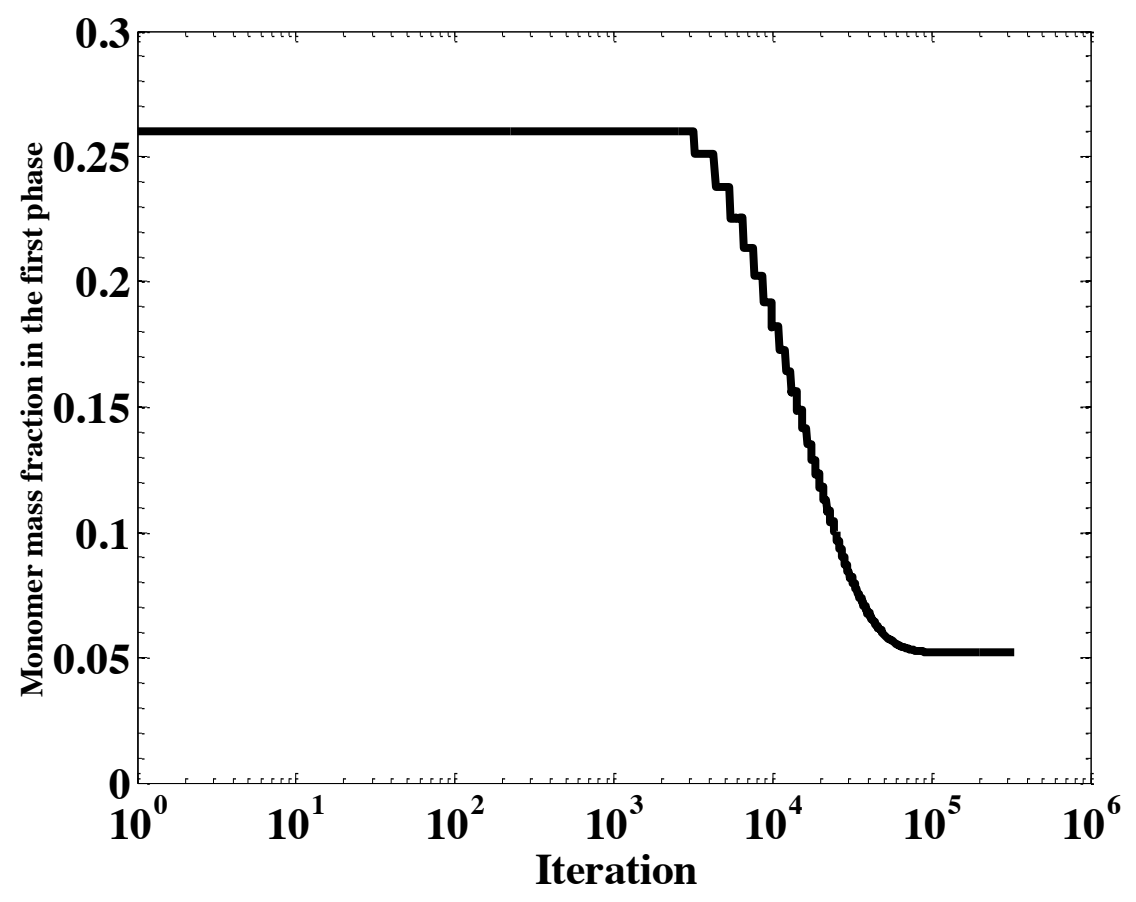

Figure 4.1-2 Monomer mass fraction convergence at the function of iteration.

As mentioned earlier, the UDF codes were introduced in transport equations after 3000 iterations. Hence the monomer mass fraction was constant. After that, the monomer mass 
fraction decreased with the iteration progress and finally reached a plateau again when the solution approached the convergence.

In addition to the above mentioned surface monitor, the transport equation residuals and sum of velocity magnitude were also monitored to keep the track of the transport equations convergence.

\subsubsection{Grid independency check}

The accuracy of the transport equation solutions depends on the grid resolution of the flow domain. The coarse grid cannot properly resolve the gradients of the flow field variable and may give misleading results. The accuracy of the solution increases with enhancement in grid resolution but the solution of the transport equations on high grid resolution requires more computational time. Hence, it becomes necessary to design a grid that is optimum compromise between desired accuracy and computational cost of the solution. In CFD analysis, it is a common practice to check the dependency of the solution on the grid resolution. For the reactor equipped with the pitched blade, 320526, 500385, and 743170 cells and for the Rushton impeller 410639, 583197, and 842850 cells were generated using ANSYS Design Modeler 14.5. The complete transport equations along with the population balance equations were solved on the three grids for both types of impellers. In the case of using the pitched blade impeller, as the axial flow was more representative variable of the flow domain, the results were compared on a line positioned below the impeller. In the case of using the Rushton impeller, as the radial flow was more typical variable, the results were analyzed on a vertical line positioned on the side of impeller. Figure 4.1-3, Figure 4.1-4, and Figure 4.1-5 show the axial velocity, turbulent kinetic energy and polymer volume fraction for the three grids for the pitched blade impeller on a line positioned at the $x=0.0, y=-0.025$ to $0.025 \mathrm{~m}$, and $z=0.11 \mathrm{~m}$ location for all the grids. The negative axial velocity represents downward velocity in the reactor. 
Figure 4.1-6, Figure 4.1-7, and Figure 4.1-8 depict the radial velocity, turbulent kinetic energy and polymer volume fraction for the three grids for the Rushton impeller on a line positioned at the $\mathrm{x}=0.03 \mathrm{~m}, \mathrm{y}=0.0 \mathrm{~m}$, and $\mathrm{z}=0.08 \mathrm{~m}$ to $0.105 \mathrm{~m}$ location for all the grids.

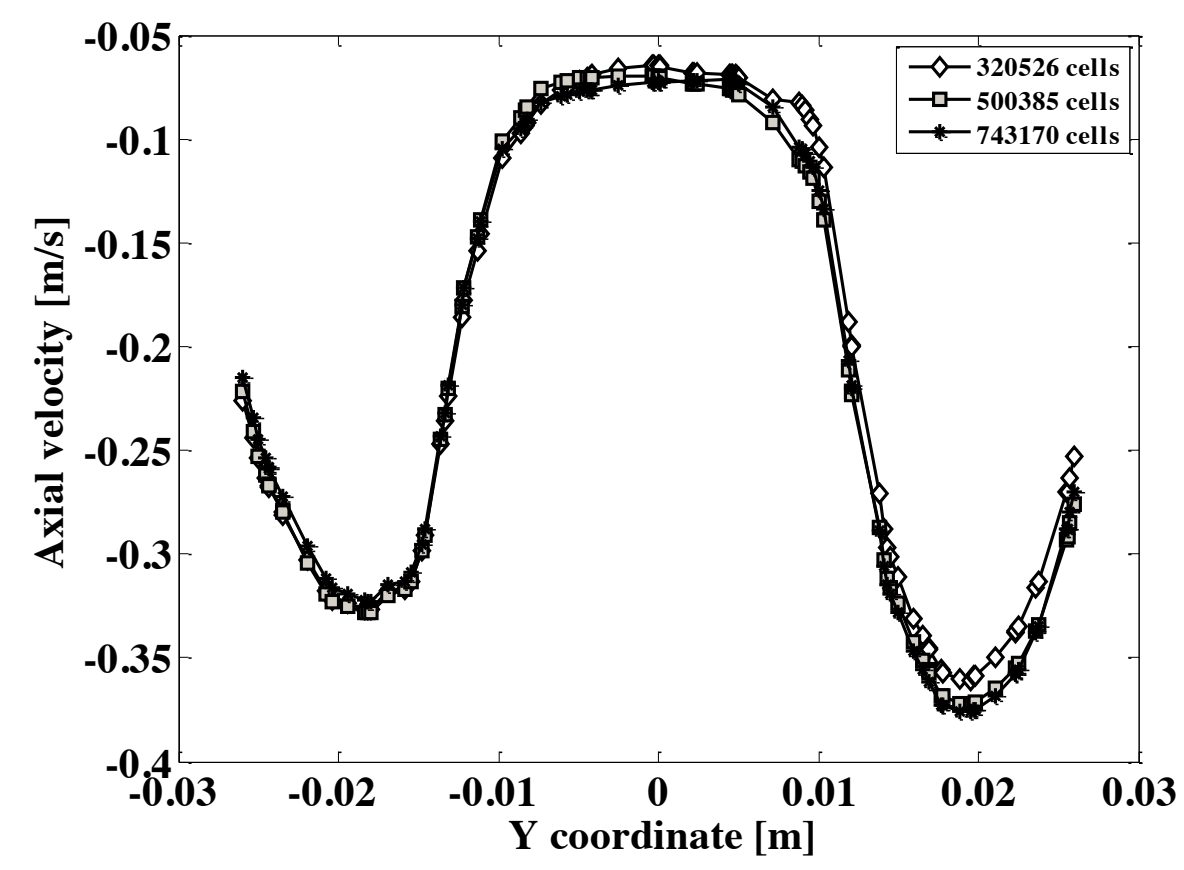

Figure 4.1-3 Grid independency study through axial velocity profile using the pitched blade impeller.

In order to quantify the discrepancy, the root mean square (RMS) deviation (Arratia et al., 2006) was calculated:

$$
R M S=\frac{\left[\left(\frac{1}{n} \sum_{i=1}^{n}\left(x_{1, i}-x_{2, i}\right)^{2}\right]^{\frac{1}{2}}\right.}{\left[\frac{1}{n} \sum_{i=1}^{n} x_{2, i}{ }^{2}\right]^{\frac{1}{2}}}
$$

where $x_{1}$ is variable on node $i$ for grid $1, x_{2}$ is variable on node $i$ for grid 2 and $n$ is number of nodes. 


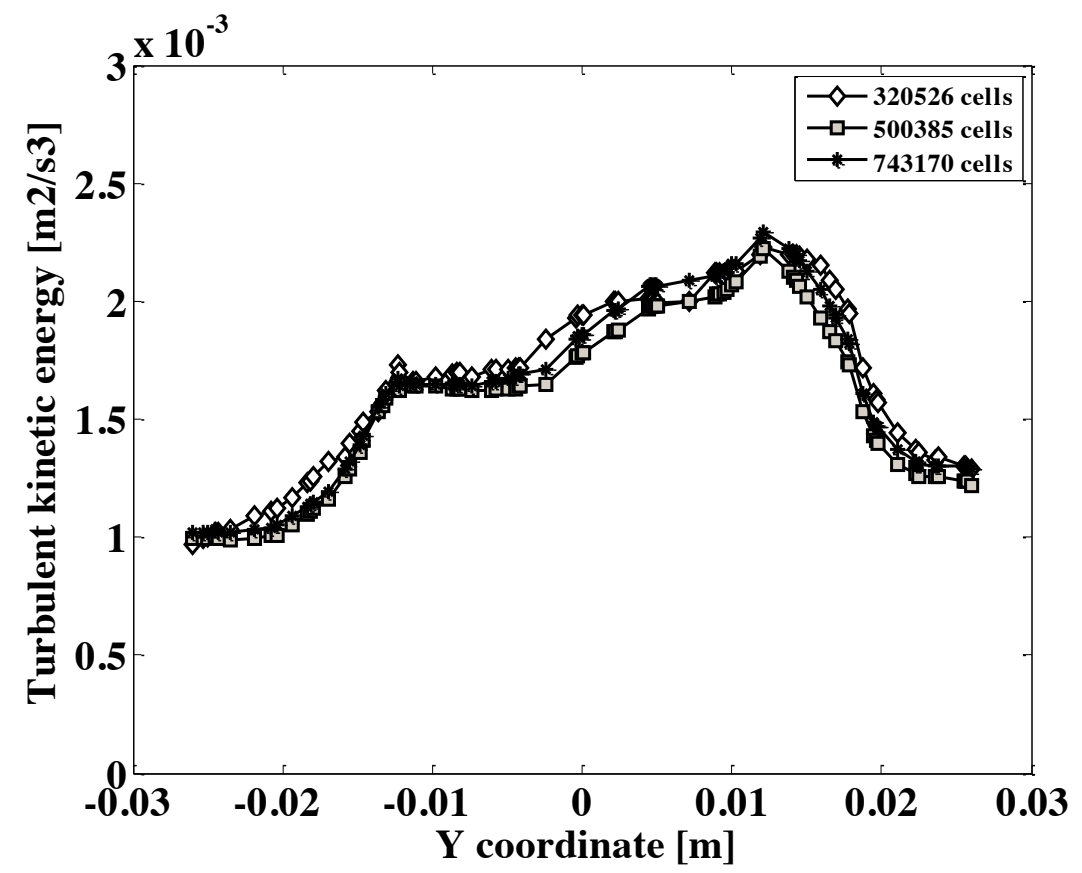

Figure 4.1-4 Grid independency study through turbulent kinetic energy profile using the pitched blade impeller.

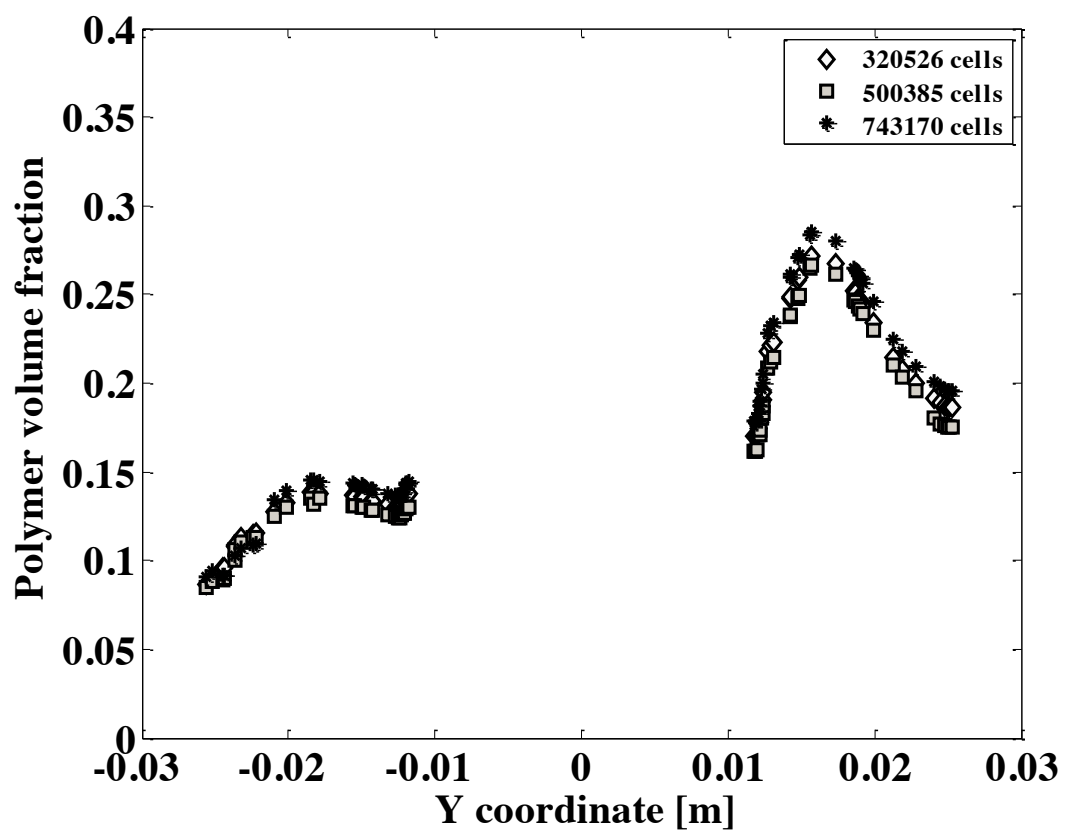

Figure 4.1-5 Grid independency study through polymer phase volume fraction profile using the pitched blade impeller. 


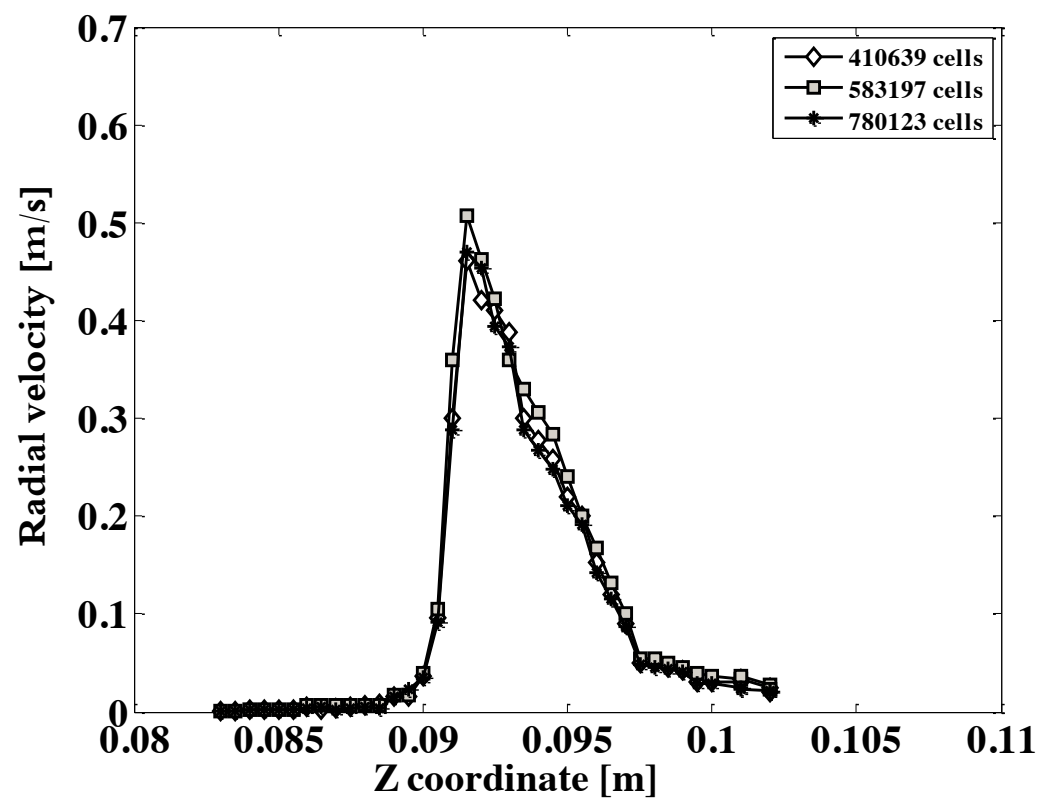

Figure 4.1-6 Grid independency study through radial velocity profile using the Rushton impeller.

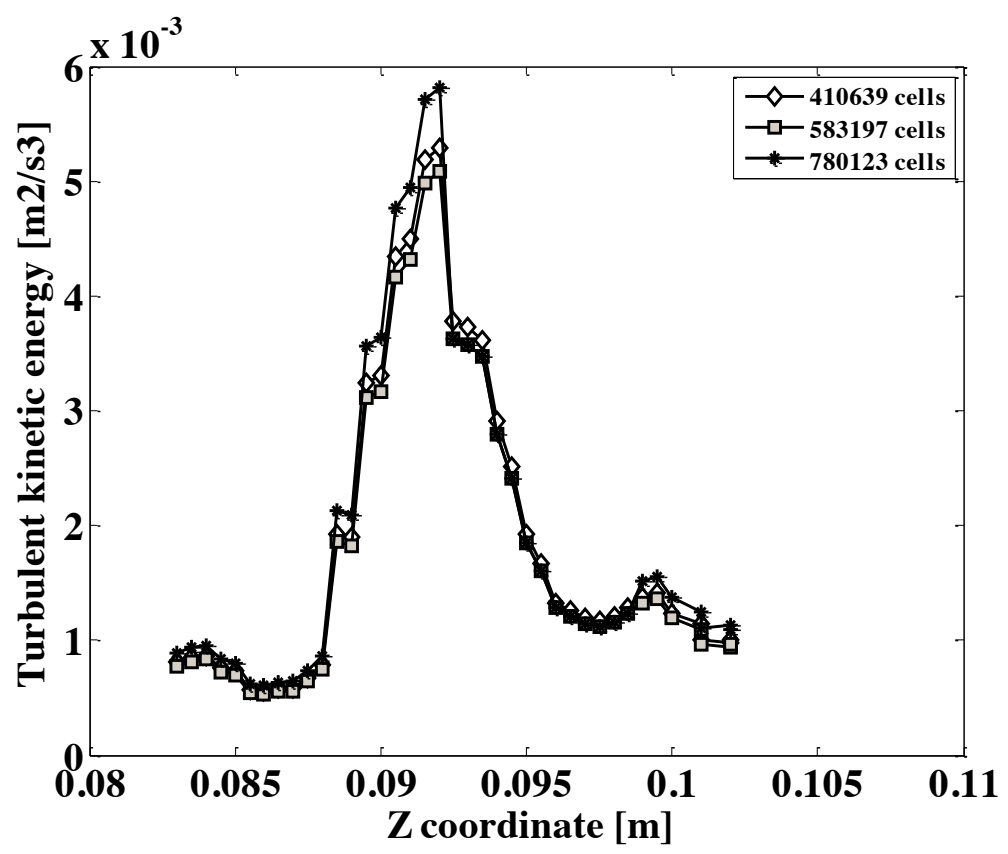

Figure 4.1-7 Grid independency study through turbulent kinetic energy profile using the Rushton impeller. 


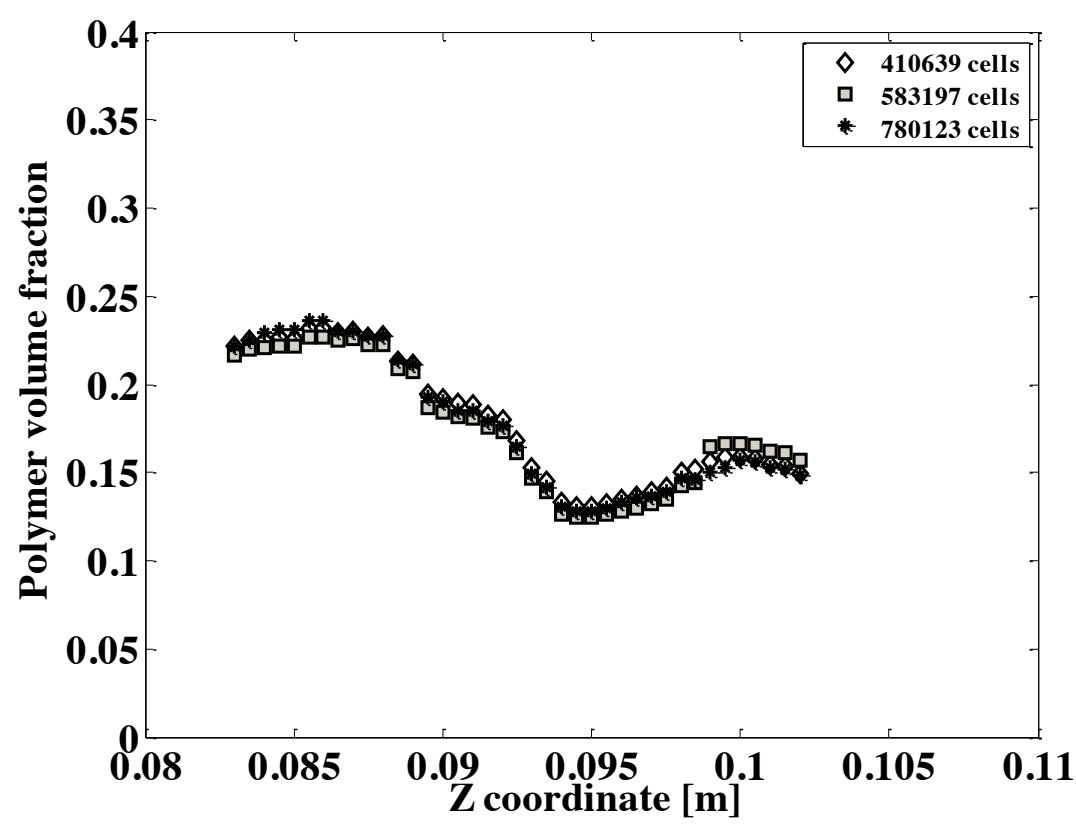

Figure 4.1-8 Grid independency study through polymer phase volume fraction profile using the Rushton impeller.

Table 4.1-3 RMS values for the reactor equipped with the pitched blade impeller at the speed of $350 \mathrm{rpm}$ for different number of cells at the location of $x=0.0, y=-0.025$ to 0.025 , and $z=0.11 \mathrm{~m}$.

\begin{tabular}{|lcc|}
\hline Variable & $\mathbf{3 2 0 5 2 6}$ and 500385 cells & $\mathbf{5 0 0 3 8 5}$ and 743170 cells \\
\hline Axial velocity & $5.1 \%$ & $4.0 \%$ \\
4.1 .5 & & \\
Turbulent kinetic energy & $4.3 \%$ & $3.5 \%$ \\
Polymer volume fraction & $4.9 \%$ & $4.2 \%$ \\
\hline
\end{tabular}


Table 4.1-4 RMS values for the reactor equipped with the Rushton impeller at the speed of 350 rpm for different number of cells at the location $x=0.03, y=0.0$, and $z=0.08$ to $0.105 \mathrm{~m}$.

\begin{tabular}{|lcc|}
\hline Variable & $\mathbf{4 1 0 6 3 9}$ and 583197 cells & $\mathbf{5 8 3 1 9 7}$ and 780123 cells \\
\hline Radial velocity & $4.7 \%$ & $4.2 \%$ \\
Turbulent kinetic energy & $4.8 \%$ & $3.9 \%$ \\
Polymer volume fraction & $4.8 \%$ & $3.8 \%$ \\
\hline
\end{tabular}

The calculated errors are shown in Table 4.1-3 and Table 4.1-4 for the reactor equipped with the pitched and Rushton impellers, respectively. All the RMS values were acceptable with the confidence in the solution accuracy as were less than 5\%. Hence, the solution of the transport equation on the coarse cells for the pitched blade (320526 cells) and Rushton impeller (410639 cells) were considered grid independent and selected for the final 3D mesh of the models. 


\subsection{CFD modeling of water and oil emulsion}

\subsubsection{Emulsion tank geometry}

The experimental data reported by Boxall et al. (2010) were used in this research to validate the CFD model. Figure 4.2-1 demonstrates the schematic diagram of the emulsion tank. The tank was a flat-bottom cylindrical tank with a diameter of $0.102 \mathrm{~m}$. The mixing vessel was equipped with a Rushton turbine impeller and four baffles spaced at $90^{\circ}$ intervals against the wall. The impeller diameter, impeller off-bottom clearance, and liquid height in the tank were $0.050 \mathrm{~m}$, $0.051 \mathrm{~m}$, and $0.098 \mathrm{~m}$, respectively. The impeller speed was varied from $300 \mathrm{rpm}$ to $600 \mathrm{rpm}$. Water and two types of crude oil (Conroe oil and Troika oil) were utilized as the dispersed and continuous phases, respectively. Physical properties of oils and water are tabulated in Table 4.2-1 (Boxall et al., 2010).
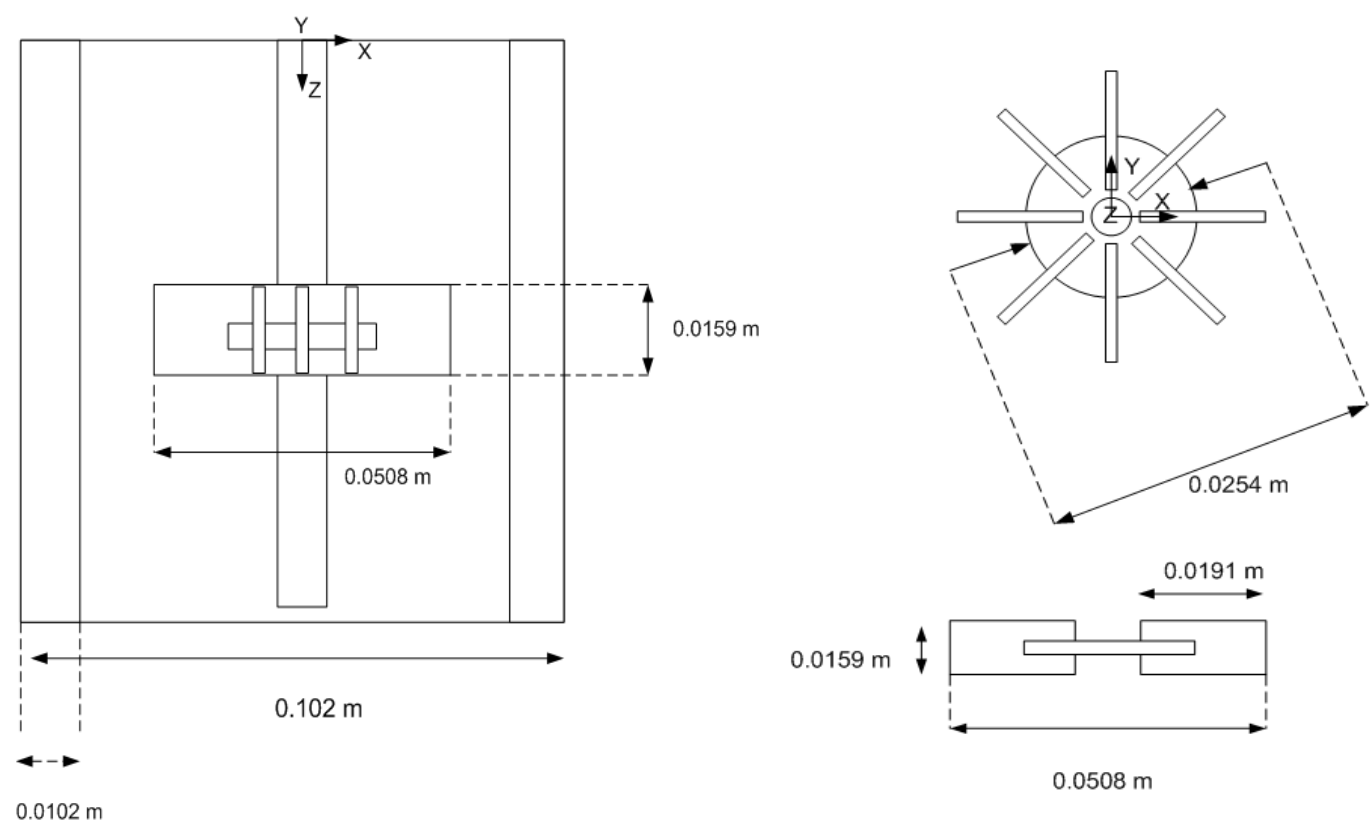

$0.0508 \mathrm{~m}$

Figure 4.2-1 Schematic diagram of water and oil tank. 
Table 4.2-1 Physical properties of fluids at $20^{\circ} \mathrm{C}$ and $1 \mathrm{~atm}$ (from Boxall et al., 2010).

\begin{tabular}{|lccc|}
\hline Fluid & Density $\left(\mathbf{k g} / \mathbf{m}^{3}\right)$ & Viscosity $(\boldsymbol{c P})$ & $\begin{array}{c}\text { Interfacial Tension } \\
\text { with water }(\boldsymbol{m} \mathbf{N} / \boldsymbol{m})\end{array}$ \\
\hline Conroe Oil & 842 & 3.1 & 20 \\
Troika Oil & 869 & 20 & 11 \\
Water & 1000 & 1 & - \\
\hline
\end{tabular}

\subsubsection{Mathematical model}

In this study, the Eulerian approach was employed to model the multiphase flow inside the emulsion tank. This technique solves a set of continuity and momentum equations for each phase. The continuity equation for phase $q$ is (Ranade, 2002):

$$
\frac{1}{\rho_{q}}\left(\frac{\partial}{\partial t}\left(\alpha_{q} \rho_{q}\right)\right)+\nabla .\left(\alpha_{q} \rho_{q} \vec{v}_{q}\right)=0
$$

where $\rho_{q}$ is the density of phase $q, \alpha_{q}$ is the phase volume fraction, and $\vec{v}_{q}$ is the velocity of phase $q$.

The momentum equation for phase $q$ is expressed as (Ranade, 2002):

$$
\begin{gathered}
\frac{\partial}{\partial t}\left(\alpha_{q} \rho_{q} \vec{v}_{q}\right)+\nabla \cdot\left(\alpha_{q} \rho_{q} \vec{v}_{q} \vec{v}_{q}\right)=-\alpha_{q} \nabla p+\nabla \cdot \overrightarrow{\vec{\tau}}_{q}+\alpha_{q} \rho_{q} g+\sum_{p=1}^{n}\left(K_{p q}\left(\vec{v}_{p}-\vec{v}_{q}\right)\right)+\vec{F}_{q} \\
\overrightarrow{\vec{\tau}}=\mu\left[\left(\nabla \vec{v}_{q}+\nabla \vec{v}_{q}^{T}\right)-\frac{2}{3} \nabla \cdot \vec{v}_{q} \overrightarrow{\vec{I}}\right]
\end{gathered}
$$


Here $g$ is the acceleration due to gravity, $\overrightarrow{\vec{\tau}}$ is the stress tensor of phase $q, P$ is the pressure, $\vec{F}$ is the external force, and $\overrightarrow{\vec{I}}$ is a unit tensor. The exchange coefficient $K_{p q}$ is already defined in previous section in equation 4.1-9 to 4.1-13. Since the lift force is insignificant compared to the drag force in emulsions (Drew and Lahey, 1993), it was ignored in this study. The effect of virtual mass forces was neglected in this study since both phases are liquids and the density of the dispersed phase (water) is larger than the density of the continuous phase (oil) (Drew and Lahey, 1993).

The equations of the $k-\varepsilon$ model used for this section are already defined in equations $4.1-18$ to 4.1-24.

The population balance equation was coupled with turbulent and multiphase flow equations to obtain the size distribution of water droplets in the continuous oil phase. The general population balance equation can be written as follows (Hagesaether et al., 2002):

$$
\frac{\partial}{\partial t}\left(\rho n_{k}^{\prime}\right)+\nabla \cdot\left(\rho \vec{v}_{q} n_{k}^{\prime}\right)=\rho\left[B_{B}-D E_{B}+B_{C}-D E_{C}\right]_{i}
$$

where $n_{k}^{\prime}$ shows the number of droplets of size $k, B_{B}$ and $D_{B}$ denote birth and death due to breakage and $B_{C}$ and $D_{C}$ denote birth and death due to coalescence of droplets. According to Hagesather et al. (2002), the birth and death rate due to breakage can be written as below:

$$
\begin{aligned}
& B_{B}(i)=\sum_{k=i+1, i \neq N}^{N} \Omega_{B}\left(v_{k}, v_{i}\right)+\sum_{k=1, i \neq N}^{i} x_{i+1, k} \Omega_{B}\left(v_{i+1}, v_{k}\right)+\sum_{k=1, i \neq 1}^{i-1}\left(1-x_{i, k}\right) \Omega_{B}\left(v_{i}, v_{k}\right) \\
& i=1, \ldots, N
\end{aligned}
$$


$\Omega_{B}\left(v_{i}, v_{k}\right)$ is the break up rate of the droplets of size $v_{i}$ into droplet $v_{k}$ and is described in equation 4.2-13. If the break up volume fraction $v_{j}$ happens, it is distributed as follows:

$$
\begin{aligned}
& v_{j}=x_{i, k} v_{i-1}+\left(1-x_{i, k}\right) v_{i} \\
& x_{i, k}=2^{1+k-i} \quad i, k=1,2,3, \ldots .
\end{aligned}
$$

The discrete method was used in this research to compute the droplet size distribution. The total number of particles per unit volume $(N)$ or number density is calculated using the following equation:

$$
N=\int n d V
$$

In the present study, two phenomena were chosen in the discrete method of population balance model: coalescence and breakage while nucleation and growth were not considered as the mass transfer between the two phases was neglected (Agterof et al., 2003, Coulaloglou and Tavlarides, 1977, Silva et al., 2008, Srilatha et al., 2010). The range of droplet sizes in the droplet size distribution was divided into a finite number of intervals or bins (classes). Generally, the size chosen for a bin is required to be in geometric progression with the adjacent bin size (Hill and $\mathrm{Ng}, 1995)$. In homogenous discrete method all bins are assigned to one dispersed phase and have the same $\vec{v}$ velocity. The general transport equation for the discrete bin (classes) fraction $f_{i}$ is:

$$
\frac{\partial}{\partial t}\left(\rho \alpha f_{i}\right)+\nabla \cdot\left(\vec{v}_{p} \alpha f_{i}\right)=\bar{S}_{i}
$$

The net mass source for the phase, $\bar{S}_{i}$, is zero as follows: 


$$
\sum_{i=1}^{L} \bar{S}_{i}=0
$$

where $L$ is the number of bins in the phase and:

$$
\sum_{i=1}^{L} f_{i}=1
$$

In the present CFD modeling, the mass conservation equation was calculated for each bin as shown by equation 20. However, for all bins, one momentum conservation equation was considered assuming that all bins were advected by the dispersed phase momentum. This was due to limitation of the homogenous discrete method (Hagesaether et al., 2002, Hounslow et al., 1988, Litster et al., 1995). On the other hand, in the inhomogeneous discrete method a separate momentum equation can be applied for each bin (Kerdouss et al., 2008). Although it would have been more accurate to utilize the inhomogeneous discrete method, it could definitely involve a more intensive CPU task, which might affect the convergence of the equations as well. To run the CFD model with the homogeneous discrete method, we employed 24 dual core processors. In case, if the inhomogeneous discrete method were used, even more processors were needed. The CFD results with the homogeneous discrete method were in very good agreement with the experimentally measured data.

In this study, the transport equations were solved for each bin, which represented the volume fraction of water phase in that bin. Thus, the total water phase volume fraction was the sum of volume fractions for all the discrete bins. The birth and death of droplets in each bin demonstrated whether they enter or leave the bin due to the breakup or coalescence. After the transport equations for the scalars were solved, the values of the number density function for 
each size bin was calculated. This was simply the volume fraction of each bin divided by the volume of a single droplet yielding the number of droplets per unit volume or number density. The values of the number density function for all bins gave the droplet size distribution. The transport equations from the population balance model and the momentum equations were coupled and in order to describe breakage and coalescence terms in equation 15, Luo's model (Luo, 1993; Luo and Svendsen, 1996) was employed. In this model, for binary breakage, size of daughter drops was calculated as:

$$
f_{B V}=\frac{D_{I}^{3}}{D^{3}}=\frac{D^{3}{ }_{I}}{D_{I}^{3}+D_{I I}^{3}}
$$

where $D_{I}$ and $D_{I I}$ are diameters of daughter droplets in the binary breakage with parent diameter of $D$. According to Luo (Luo and Svendsen, 1996) only eddies smaller than or equal to the droplet can make particle to oscillate. If we consider the variable $\xi=\lambda / D$ as a dimensionless eddy size, $D$ as droplet diameter, and $\lambda$ as eddy size, then the equation for breakage rate is expressed as:

$$
\Omega_{B}\left(v_{i}, v_{k}\right)=K \int_{\xi_{\min }}^{1} \frac{(1+\xi)^{2}}{\xi^{11 / 3}} \exp \left(-b \xi^{-11 / 3}\right) d \xi
$$

where the parameters are:

$$
\begin{gathered}
K=0.923 \varepsilon^{1 / 3} D^{-2 / 3} \alpha \\
b=12 c_{f} \sigma \rho^{-1} \varepsilon^{-2 / 3} D^{-5 / 3} \\
c_{f}={f_{B V}}^{2 / 3}+\left(1-f_{B V}\right)^{2 / 3}-1
\end{gathered}
$$


The birth and the death of the droplets due to coalescence are:

$$
\begin{gathered}
B_{C}(i)=\sum_{j=1, i \neq N}^{i-1} x_{i, j} \Omega_{C}\left(v_{i}, v_{j}\right)+\sum_{j=1}^{i-1}\left(1-x_{i-1, j}\right) \Omega_{C}\left(v_{i-1}, v_{j}\right), \quad i=2, \ldots, N \\
D E_{C}(i)=\sum_{j=1}^{N-1} \Omega_{C}\left(v_{i}, v_{j}\right)+\Omega_{C}\left(v_{i}, v_{j}\right), \quad i=2, \ldots, N
\end{gathered}
$$

The coalescence term $\Omega_{C}\left(v_{i}, v_{j}\right)$ is the rate of coalescence between droplets $v_{i}$ and $U_{j}$ :

$$
\begin{gathered}
v_{i}+v_{j}=x_{i, j} v_{i}+\left(1-x_{i, j}\right) v_{i+1} \\
x_{i, j}=1-2^{j-i}, i \geq j
\end{gathered}
$$

Details of Luo's coalescence equations for description of $\Omega_{C}\left(\nu_{\left.i, \nu_{j}\right)}\right.$ can be found elsewhere (Luo, 1993).

It should be mentioned that the computational approach in Schütz et al. (2009) paper was found to be similar to our study. However, they applied the CFD technique for a different process, i.e. hydrocylcone separation. In addition, for multiphase modeling, we employed Eulerian approach, which is more accurate compared to mixture model (Ansys Fluent 12.0, 2009) used by Schütz et al. (2009) for multiphase modeling. The mathematical equations including multiphase motion, turbulence, breakage and coalescence equations were coupled with the discrete method in population balance approach and were employed in the CFD simulations. 


\subsubsection{CFD simulation}

Gambit 2.4 was utilized to discretize the 3D computational domain via unstructured tetrahedral cells (Figure 4.1-2). To capture the flow details near the flow domain boundaries, a size function was employed to generate very refined mesh in the vicinity of the rotating impeller and the tank wall. To test for grid independence, the mesh was fine-tuned based on the velocity profiles computed near the impeller, where is the region of large velocity gradients. Grid independence was achieved when the additional cells did not change the calculated velocity near the impeller blades by more than $5 \%$. The final three-dimensional model had 299,616 cells.

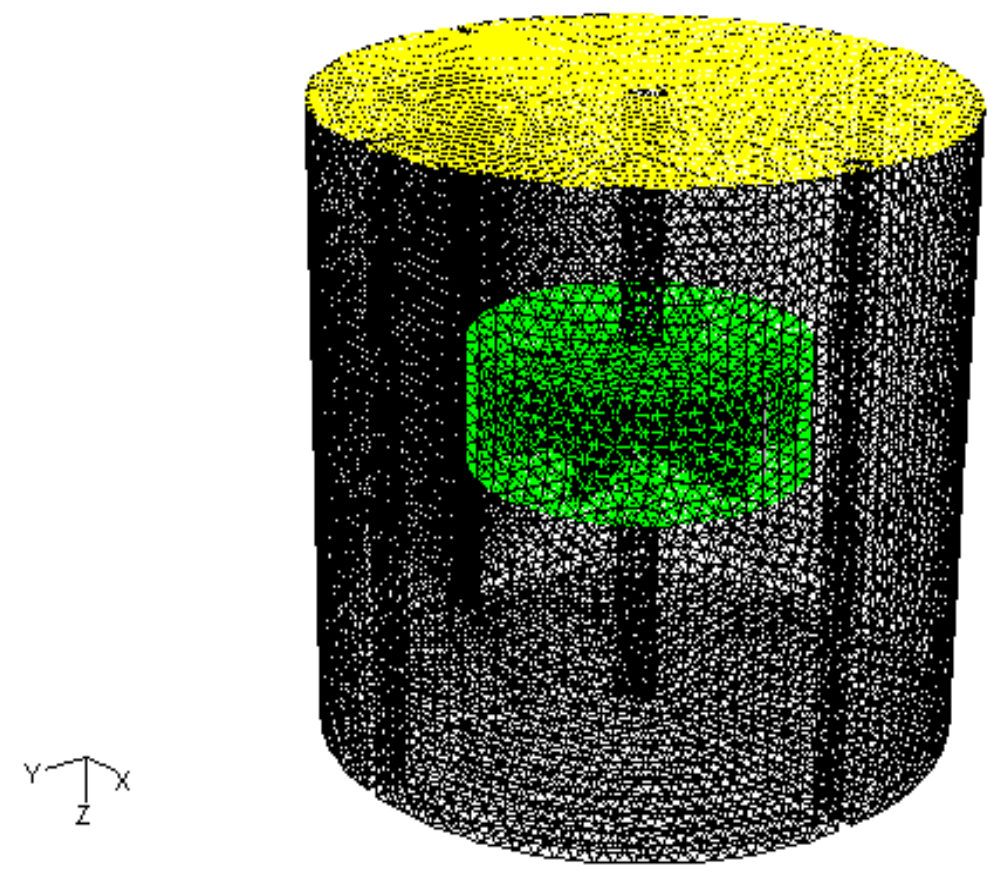

Figure 4.2-2 Geometry grid of water and oil emulsion system. 
According to the literature, the rotation of an impeller in a tank can be modeled by several techniques such as multiple reference frames (MRF) and sliding mesh (SM) methods (Deen et al., 2002; Brucato et al., 1998). The MRF approach predicts the flow field for a fixed position of the impeller relative to the baffles (Luo et al., 1994). In the sliding mesh approach (Luo et al., 1993), the dynamic simulations are carried out using two grid zones; one is attached to the baffles and tank wall while the other is attached to the rotating impeller. As the impeller rotates in time relative to the baffles, so does the grid in the impeller region, while the baffle region does not move. To evaluate the motion of the grid, the solution method must be time-dependent. The SM approach is more accurate but it requires more CPU time than that of the MRF approach (Mostek et al., 2005). Several researchers have used MRF method for the similar mixing geometry and achieved good results (e.g. Kerdouss et al., 2008; Pakzad et al., 2008; Patel et al., 2010a and 2010b). In this study, we employed MRF technique to capture the motion of the rotating impeller in a stationary tank and the CFD results matched very well with the experimental data. Therefore, to reduce the computational time, we did not utilize the sliding mesh technique. The transport equations inside and outside the rotating frame were solved separately and as the solution proceeded a transfer of information was considered at the interface.

Fluent package (version 6.3.26) was employed to solve all transport equations for unsteady-state condition. All transport equations were integrated using control volume method. Standard wall function was considered as near wall treatment (Launder and Spalding, 1974). The distance between the first cell and the wall was bridged by wall functions (Blazek, 2005). On the liquid level, the symmetry boundary condition was used. The flow was considered turbulent with a maximum Reynolds number of approximately 5000. First-order upwind discretization scheme 
was employed to calculate the face fluxes in the momentums and phase transport equations. The velocity-pressure coupling was solved using phase coupled SIMPLE algorithm (Patanakar and Spalding, 1972). Integrating the governing transport equations over small control volumes resulted in an algebraic linear system, which was solved with the Gauss-Seidel iterative method and the Algebraic Multi-Grid method (AMG). The reactor model was comprised of continuity equation, three momentum equations, four volume fraction transport equations for the two phases, two $k$ and $\varepsilon$ turbulence equations, and seven equations for the bin fractions of the second phase. The simulation started with all velocity components set to zero. This condition simulated the impeller-stirred tank to be initially at rest. Therefore, water as the second phase with the volume fraction of 0.15 or 0.25 was at the lower section of tank, below the continuous phase, due to the higher density of water compared to the densities of Conroe oil and Troika oil as tabulated in Table 1. All equations were simultaneously solved in unsteady state mode with the step size of $0.01 \mathrm{~s}$, number of time steps of 5000, and maximum iteration per time step of 30 (Harvey and Rogers, 1996). The initial condition for the turbulent energy dissipation $\varepsilon_{t u r b}$ was $1 \mathrm{~m}^{2} / \mathrm{s}^{3}$ and for the turbulent kinetic energy $k_{\text {turb }}$ was equal to $1 \mathrm{~m}^{2} / \mathrm{s}^{2}$. The geometric ratio between seven bin sizes was equal to one and was chosen in such a way that the Sauter mean diameter was located in Bin 3. The bin sizes at different impeller speeds and for two types of oils are tabulated in Table 4.1-2. Initial volume fraction of all bins to dispersed phase were set to 0.1 except for the Bin 3 whose volume fraction was assumed to be 0.4 (The sum of all bin volumes divided by the dispersed phase volume was equal to one.). 
Table 4.2-2 Diameter of the drop sizes used in discrete method.

\begin{tabular}{|c|c|c|c|c|}
\hline \multirow{2}{*}{ Bin } & size $\mu m(300 \mathrm{rpm})$ & size $\mu m(400 \mathrm{rpm})$ & size $\mu m(600 \mathrm{rpm})$ & size (300 rpm) \\
\hline & & Conroe Oil & & Troika Oil \\
\hline 1 & 280 & 208 & 132 & 160 \\
\hline 2 & 222 & 165 & 104 & 126 \\
\hline 3 & 176 & 131 & 83 & 100 \\
\hline 4 & 140 & 104 & 66 & 80 \\
\hline 5 & 111 & 82 & 52 & 63.4 \\
\hline 6 & 88 & 65.5 & 41 & 50 \\
\hline 7 & 70 & 52 & 33 & 40 \\
\hline
\end{tabular}

Convergence was checked by monitoring the residuals of all mentioned equations, sum of velocity magnitude at the liquid surface, and the water phase bin fractions average. Supercomputing facilities of HPCVL (High Performance Computing Virtual Laboratory) was utilized to perform the computations. Each simulation was run in parallel with 24 dual cores SUN Ultra-Spark IV, 1.8GHz Sun Micro-Systems CPUs. The grid was partitioned into 24 parts and one CPU was then assigned to each partition. The convergence for each unsteady state simulation was achieved after 8 hours for 7 droplet bin size fractions (classes) in discrete model. 


\subsection{Modeling of MMA polymerization in a CSTR}

\subsubsection{Reactor system}

Figure 4.3-1 shows a schematic diagram of the CSTR reactor considered in this CFD study. The reactor is a round bottom cylindrical tank with a diameter of $10.16 \mathrm{~cm}$ and a height of $13.46 \mathrm{~cm}$. The vessel was equipped with a $45^{\circ}$ pitched bladed turbine impeller with a diameter and offbottom clearance of $5.08 \mathrm{~cm}$ and $3.69 \mathrm{~cm}$, respectively. The impeller speed was varied up to 500 rpm. The reaction system consists of methyl methacrylate (MMA), ethyl acetate $\left(\mathrm{EA}_{\mathrm{c}}\right)$ and azobis isobutyronitrile (AIBN), which are, respectively, the monomer, solvent and chemical initiator species. These reactants were fed to reactor. The polymer, un-reacted monomer, and solvent were withdrawn from the bottom of tank. The CFD modeling approach is described next.

\subsubsection{Mathematical model}

The reactor model consisted of a transport model coupled with a polymerization kinetic model. Assuming a laminar flow steady state condition, the governing transport equations of continuity, momentum, and species balance are as follows (Serra et al., 2007):

Continuity:

$$
(\nabla \cdot \rho \vec{v})=0
$$

where $\rho$ is the density and $\vec{v}$ is the velocity vector.

Momentum balance:

$$
\nabla \cdot(\rho \vec{v} \vec{v})=-\nabla P+(\nabla \cdot \overrightarrow{\vec{\tau}})+\rho \vec{g}+\vec{F}
$$


$P$ is the pressure, $\vec{g}$ is the gravitational force, $\overrightarrow{\vec{\tau}}$ is the stress tensor described in equation $4.2-3$, and $\vec{F}$ is the external force.

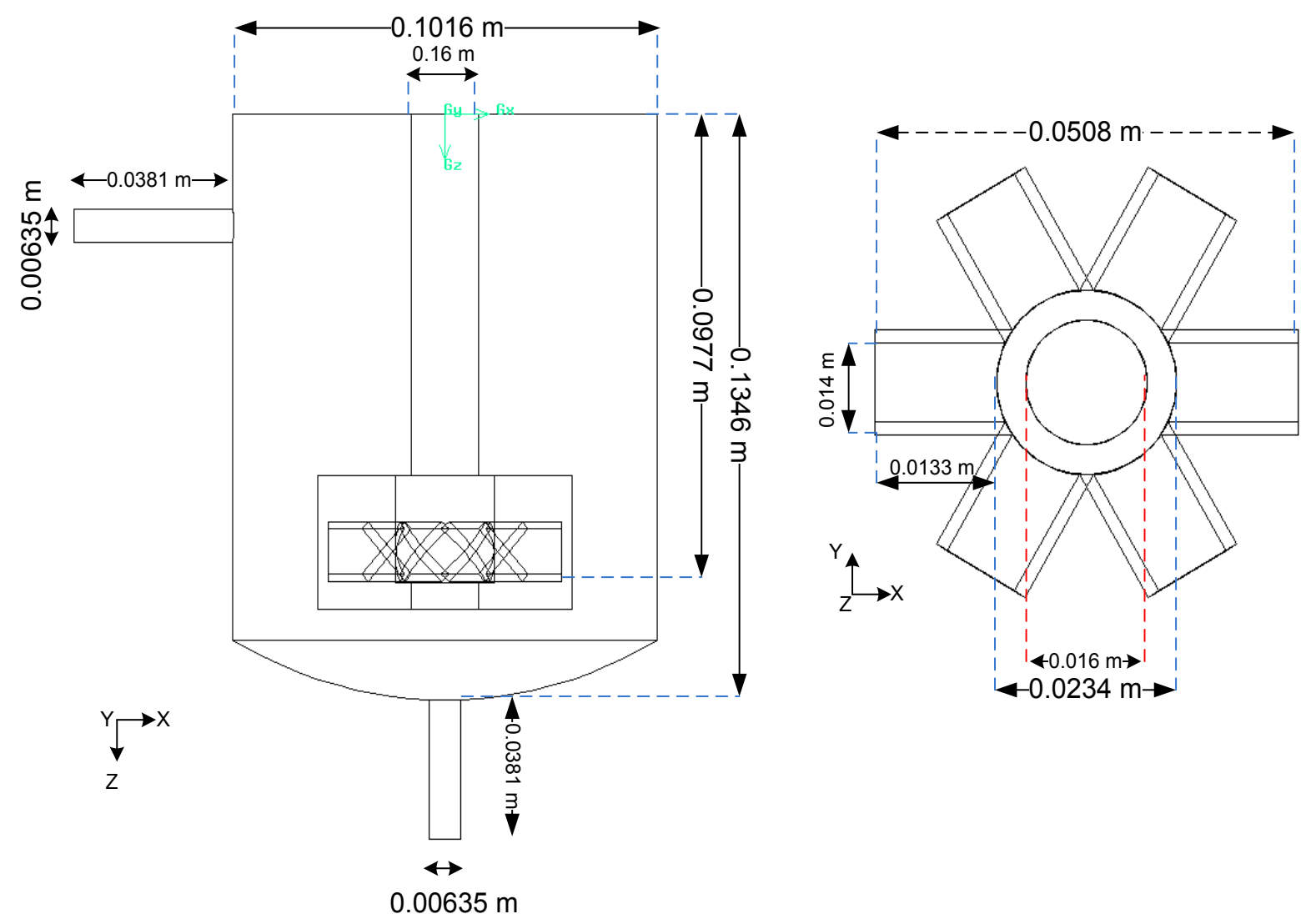

Figure 4.3-1 Schematic diagram of CSTR.

Species balance:

$$
\nabla \cdot\left(\rho \vec{v} W_{i}\right)=-\nabla \cdot \vec{J}_{i}+\bar{S}_{i}
$$

where

$$
\vec{J}_{i}=-\rho(D I F)_{i} \nabla W_{i}
$$


$J_{i}, D I F_{i}, W_{i}$, and $\bar{S}_{i}$ are diffusive flux, mass diffusivity, mass fraction, and mass source, respectively that are related to the $i^{t h}$ species. Physical properties of species are tabulated in Table 4.3-1.

Table 4.3-1 Physical properties of reactor species at $20^{\circ} \mathrm{C}$ and $1 \mathrm{~atm}$.

\begin{tabular}{|lccc|}
\hline Fluid & Density $\left(\mathbf{k g} / \mathbf{m}^{\mathbf{3}}\right)$ & Viscosity $(\mathbf{P a} . \mathbf{s})$ & $\mathbf{M}_{\mathrm{w}}(\mathrm{g} / \mathbf{m o l})$ \\
\hline Methyl methacrylate (MMA) & 895 & $3.7 \mathrm{e}-4$ & 100.12 \\
Azobisisobutyronitrile (AIBN) & 1100 & $2.8 \mathrm{e}-4$ & 164 \\
Ethyl Acetate (EA $)$ & 866 & $5.9 \mathrm{e}-4$ & 92.07 \\
\hline
\end{tabular}

\subsubsection{Reactive source term}

In this study, initiation mechanism by both chemical and thermal initiations was taken into account and the overall kinetic mechanism consisting initiation, propagation, chain transfer agent (monomer, solvent), termination by combination and disproportionation is given below (Rudin and Choi, 2012):

Thermal Initiation:

$$
3 M \stackrel{k_{t h}}{\longrightarrow} 2 R_{1}^{\bullet}
$$

Chemical Initiation:

$$
\begin{array}{r}
I \stackrel{k_{d}}{\longrightarrow} 2 R_{\text {in }}^{\bullet} \\
R_{\text {in }}^{\bullet}+M \stackrel{k_{\text {in }}}{\longrightarrow} R_{1}^{\bullet}
\end{array}
$$

Propagation:

$$
R_{r}^{\bullet}+M \stackrel{k_{\text {prop }}}{\longrightarrow} R_{r+1}^{\bullet} \quad r \geq 1
$$

Transfer to monomer

$$
R_{r}^{\bullet}+M \stackrel{k_{t r f m}}{\longrightarrow} R_{1}^{\bullet}+P_{r}
$$


Transfer to solvent

$$
R_{r}^{\bullet}+S \stackrel{k_{u f s}}{\longrightarrow} S^{\bullet}+P_{r}
$$

Termination

$$
\begin{gathered}
R_{r}^{\bullet}+R_{s}^{\bullet} \stackrel{k_{t c}}{\longrightarrow} P_{r+s} \quad r, s \geq 1 \\
R_{r}^{\bullet}+R_{s}^{\bullet} \stackrel{k_{t d}}{\longrightarrow} P_{r}+P_{s} \quad r, s \geq 1
\end{gathered}
$$

where $M, R^{\circ}$, and $P$ stand for monomer, live polymer radical and dead polymer, respectively. The subscripts $r$ and $s$ are polymer chain lengths and the reaction rate constants $k$ are provided in the nomenclature. Assuming a steady-state hypothesis and equating the rate of chemical initiation to that of termination, the overall growing polymer concentration is given by (Kolhapure and Fox, 1999):

$$
\left[R^{\bullet}\right]=\sqrt{\frac{2 k_{t h}[M]^{3}+2 f k_{d}[I]}{k_{t, 0}}}
$$

where $f$ is the initiator efficiency which stands for the fraction of initiator amount that has been consumed. The values of the reaction rate constants (Schmidt and Ray, 1981; Maschio and Moutier, 1989; Choi, 1986; Dehnavi et al., 2008) are listed in Table 4.3-2. 
Table 4.3-2 Values of kinetic parameters for free radical polymerization of MMA.

$$
\begin{aligned}
& k_{t h}=1480.3 \exp \left(-138 /\left(R_{g} T\right)\right)^{1)} \\
& k_{d}=1.33 \times 10^{15} \exp \left(-30700 /\left(R_{g} T\right)\right)^{2)} \\
& k_{\text {prop }}=4.41 \times 10^{5} \exp \left(-4350 /\left(R_{g} T\right)\right)^{2)} \\
& k_{t r f m}=4.67 \times 10^{-2} \exp \left(-888 /\left(R_{g} T\right)\right)^{2)} \\
& k_{t r f s}=6.55 \times 10^{14} \exp \left(-24000 /\left(R_{g} T\right)\right)^{2)} \\
& k_{t, 0}=6.5 \times 10^{7} \exp \left(-700 /\left(R_{g} T\right)\right)^{2)} \\
& k_{t, 0}=k_{t c}+k_{t d} \\
& f=0.4^{2)}
\end{aligned}
$$

$K_{t, 0}$ is the initial total termination rate constant as described below:

$$
K_{t, 0}=K_{t c}+K_{t d}
$$

To account for the auto-acceleration phenomenon in the kinetics model, the approach of the free volume theory is considered. The free volume $v_{f}$ of the solution polymerization system is expressed as (Schmidt and Ray, 1981):

$$
v_{f}=v_{f, m} \phi_{m}+v_{f, s} \phi_{s}+v_{f, p} \phi_{p}
$$

\footnotetext{
1) Lingnau and Meyerhoff (1984)

2 ) Maschio and Moutier (1989)
} 
where $\phi_{m}, \phi_{s}$ and $\phi_{p}$ are the volume fractions and $v_{f, m}, v_{f, s}$ and $v_{f, p}$ are the specific free volume of the monomer, solvent, and polymer, respectively. They are given by (Schmidt and Ray, 1981):

$$
\begin{gathered}
v_{f, m}=0.025+0.001\left(T-T_{g m}\right) \\
v_{f, s}=0.025+0.001\left(T-T_{g s}\right) \\
v_{f, p}=0.025+0.00048\left(T-T_{g p}\right)
\end{gathered}
$$

where $T$ is the reaction temperature; $T_{g p}$ the glass transition temperature of polymer; and $T_{g m}$ and $T_{g s}$ are analogous temperatures (D'Hooge et al., 2010) for monomer and solvent, respectively (Schmidt and Ray, 1981).

The mobility of a chain is greatly reduced in the case of smaller free volume values. The onset of the gel effect may occur in the range of $20 \%$ and $40 \%$ conversion (Louie et al., 1985). In this study, it was assumed that the gel took effect for conversion higher than $30 \%$ as reported (Maschio and Moutier, 1989). Thus, the termination rate constant is given by:

$$
K_{t}=g_{t} K_{t, 0}
$$

where

$$
g_{t}=0.10575 \exp \left(17.15 v_{f}-0.01715(T-273.16)\right)
$$

The initiation rate $R_{\text {in }}$ and the polymerization rate $R_{\text {prop }}$ per unit volume are, respectively, given by:

$$
\begin{gathered}
R_{\text {in }}=2 f K_{d}[I] \\
R_{\text {prop }}=\left(K_{\text {prop }}+K_{t r f m}+K_{t r f s}\right)[M]\left[R^{\bullet}\right]
\end{gathered}
$$


The kinetic polymerization model expressed by initiation and propagation rates is linked to the hydrodynamic model via the source term which is required in Equation 4.3-3. Thus, the species transport source terms (monomer and initiator consumption rates) are defined as (Kolhapure and Fox, 1999):

$$
\begin{aligned}
& \bar{S}_{m}=R_{p r o p} \times M_{w, m} \\
& \bar{S}_{i n}=R_{i n} \times M_{w, i n}
\end{aligned}
$$

where $M_{w, m}$ and $M_{w, i n}$ are the monomer and initiator molar masses, respectively.

\subsubsection{CFD simulation}

For the CFD simulation, Gambit 2.4 was first utilized to make the reactor geometry and discretize the 3D computational domain via unstructured tetrahedral cells as shown in Figure 4.32. To capture the flow details near the flow domain boundaries, a size function was employed to generate very refined mesh in the vicinity of the rotating impeller and the tank wall. After applying the grid independence test, the final three-dimensional model had 315,087 cells. A rotating frame surrounding the impeller was generated to simulate the impeller rotation (Patel et al., 2010a and 2010b). This rotating frame was used for the region containing the impeller while a stationary frame was employed for the rest of the flow domain. The transport equations inside and outside the rotating frame were solved separately and as the solution proceeded a transfer of information was considered at the interface. ANSYS Fluent package (version 13.00) was employed to solve all transport equations at steady-state condition. A user defined function (UDF) code was written for the source terms in $\mathrm{C}$ language and linked to the species transport model coded in FLUENT. 


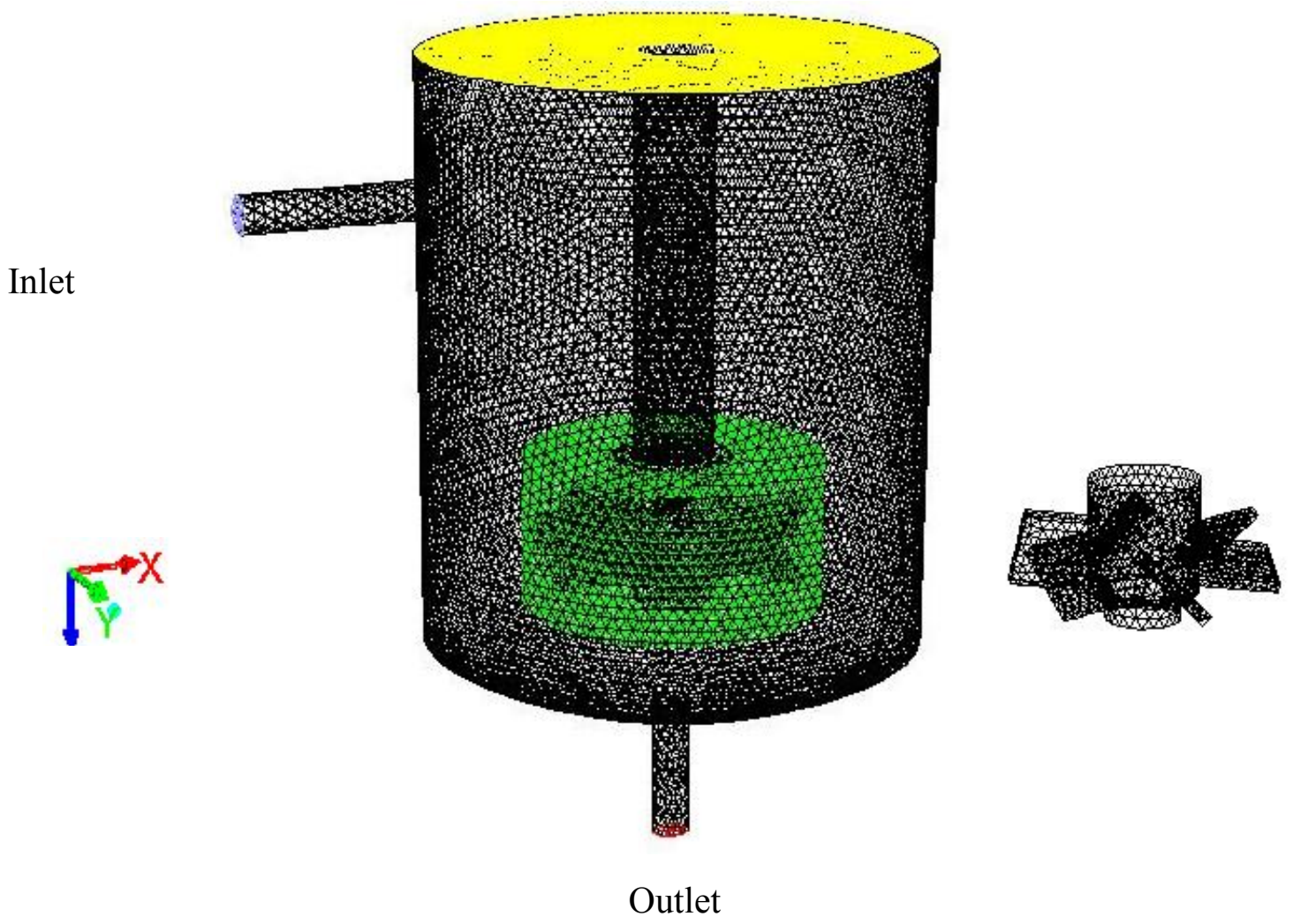

Figure 4.3-2 Geometry Grid.

All transport equations were integrated using the control volume method. In particular, species transport equations were solved for monomer, initiator, and solvent. The polymer mass fraction was calculated by subtracting the monomer and initiator mass fractions from unity in the CFD simulation. On the liquid level, a symmetry boundary condition was considered. At the feed stream of the reactor, the inlet-velocity boundary condition was selected. The inlet velocity and the inlet mass fraction of species were supplied. At the reactor outlet, zero normal gradients were used so that there was no diffusive flow normal to the boundary. On the tank walls, no slip condition was imposed on the transport equations. The flow was considered laminar with a maximum Reynolds number of approximately 1500 . The reactor was considered operating under isothermal conditions, thus a constant temperature was considered on the reactor walls. A 
second-order upwind discretization scheme was employed to determine the face fluxes in the momentum and species transport equations. The PRESTO scheme was employed for the pressure discretization, and velocity-pressure coupling was solved using phase coupled SIMPLE algorithm (Poubel et al., 2010). Integrating the governing transport equations over small control volumes resulted in an algebraic linear system, which was solved with the Gauss-Seidel iterative method and the Algebraic Multi-Grid method (AMG).

As previously mentioned, the reactor model was comprised of transport and kinetic models. First, the continuity and momentum equations were solved for an inert medium consisting of methyl methacrylate, initiator and solvent with no possibility of a reaction. After reaching a partial convergence for flow equations, the species polymerization reaction source terms were attached to Fluent and the complete model was solved. As the number of iterations was increased in simulation, the under-relaxation for the species transport equations was gradually increased to obtain a stable and accurate numerical solution. Convergence was not straightforward and three criteria were monitored to obtain when: i) the residuals of the transport equations were less than $1 \times 10^{-6}$, ii) the un-reacted monomer fraction in the outlet of reactor was invariant with iteration, and iii) the sum of velocity magnitude at the liquid surface did not vary with increased iteration. All equations were solved at the steady-state mode and convergence was achieved after 255,000 iterations. Supercomputing facilities of HPCVL (High Performance Computing Virtual Laboratory) was utilized to perform the computations. Each simulation was run in parallel with 36 dual cores SUN Ultra-Spark IV, 1.8GHz Sun Micro-Systems CPUs. The grid was partitioned into 36 parts and one CPU was then assigned to each partition. The convergence for each simulation was achieved after 5-6 days. 


\section{Chapter 5}

\section{RESULTS AND DISCUSSION}

In this chapter, all results and related discussions are presented in the five separate sections:

\section{Section 5.1: Mixing effect on emulsion polymerization in a batch Reactor}

In this section, the experimental results regarding the effects of impeller speed, mounting baffles, and reaction temperature on the monomer conversion, polymer particle size and number, and molecular weight are provided and discussed. The results presented in this section have been published in a peer-reviewed journal (Fathi Roudsari et al., 2014a).

\section{Section 5.2: Impact of impeller type on MMA emulsion polymerization in a batch reactor}

In section 5.2, the impacts of the impeller type $\left(45^{\circ}\right.$ six pitched-blade turbine and Rushton impeller), number of impellers (single and double impellers), and impeller speed (100-350 rpm) on the monomer conversion, polymer particles size, molecular weight, and glass transition temperature are analyzed through the experimental data. The results presented in this section have been published in a peer-reviewed journal (Fathi Roudsari et al., 2014b).

\section{Section 5.3: CFD Analysis of mixing in emulsion polymerization of MMA}

A novel computational fluid dynamics (CFD) model is presented for the emulsion polymerization reactor in this section. The novelty of this study was the inclusion of the chemical reaction kinetics to the population balance through nucleation and growth rates while taking into account the velocity gradients in the emulsion polymerization reactor. The mean particle size, 
conversion and number density distribution were computed using the model and were validated with the experimentally determined values. The results presented in this section have been submitted to a refereed journal.

\section{Section 5.4: CFD modeling of the mixing of water in oil emulsion}

Section 5.4 deals with the development of a CFD model for the mixing of water in oil emulsion in a lab-scale mixing tank equipped with a Rushton turbine impeller. To achieve this goal, the droplet size distribution within the mixing tank was estimated by means of the population balance approach, which employs the discrete method to describe coalescence and breakage of water droplets. In this study the incorporation of the population balance equations into the CFD model for a non-reactive emulsion system was investigated. The results presented in this section have already been published in a refereed journal (Fathi Roudsari et al., 2012).

\section{Section 5.5 Use of CFD in modeling MMA solution polymerization in a CSTR}

This modeling study was also performed to find an efficient technique to couple the reaction kinetics with the CFD model for a polymerization system inside a stirring reactor. A CSTR tank was selected to enable solution of the transport equations in the steady state mode. A computational fluid dynamics (CFD) model was developed to study methyl methacrylate (MMA) solution polymerization in a lab-scale stationary continuous stirred tank reactor (CSTR) equipped with a six bladed $45^{\circ}$ pitched. The effects of the impeller speed, reaction temperature, residence time and inlet monomer concentration on the conversion and homogeneity of reaction mixture inside the reactor were studied. The results discussed in this section have been published in a peer-reviewed journal (Fathi Roudsari et al., 2013). 


\subsection{Mixing effect on emulsion polymerization in a batch reactor (Fathi Roudsari et al., 2014a)}

\subsubsection{Introduction}

Even though significant advances have been achieved in emulsion polymerization in recent decades, the basic concept of mixing which is the basis for the formation of emulsion mixture has not been fully delineated yet. In this study, the impact of the stirring rate, baffles, and reaction temperature on the monomer conversion, the mean particle size, and the average molecular weight were extensively explored. The experimental set up was a 2L PARR reactor as shown in Chapter 3 ( Figure 3.1-1) and the experimental procedure are described in Section (3.2). In this study, the pitched blade impeller was utilized. The experimental design is shown in Table 3.4-2.

\subsubsection{Results and discussion}

In this research, the final number of particles, $N_{p}$ was calculated using the following equation (Sajjadi and Jahanzad, 2003):

$$
N_{P}=\frac{6 M_{t} x_{f}}{\pi \rho_{P} D_{P}^{3}}
$$

where $M_{t}$ is the total amount of monomer and polymer present in the reactor, $x_{f}$ is the final weight ratio of the polymer in the reactor to the total amount of monomer fed into the reactor by the time $t, \rho_{P}$ is the density of polymer, and $D_{P}$ is the volume average diameter of particles.

In our study, the experiments were performed from low to high agitation rates at a fixed chemical recipe. Sampling was started 5 min after adding initiator at $5^{\circ} \mathrm{C}$ below the set point and 
a total of 13 samples were taken for each run. Figure 5.1-1 (both plots) shows monomer conversion as a function of reaction time at different impeller speeds of 20, 100, 250, and 350 $\mathrm{rpm}$, with and without baffles at the isothermal reactor temperature of (a) $50^{\circ} \mathrm{C}$ and (b) $60^{\circ} \mathrm{C}$. According to literature (Feng et al., 2010), the induction time is the period before the reaction takes place. Therefore, in our experiments, even though the sampling was started after 5 min of adding the initiator, the conversion results, revealed that the experiments performed at lower impeller speeds (20-100 rpm) had longer induction time before the monomer conversion was raised compared to the runs at the higher agitation rates $(250-350 \mathrm{rpm})$.

At the impeller speed of $20 \mathrm{rpm}$, the very low conversion indicates that pooling of monomer occurred on top of the reaction mixture. This was predictable due to the insufficient mixing at this low agitation rate. The conversion increased when the impeller speed was varied from 20 $\mathrm{rpm}$ to $250 \mathrm{rpm}$. However, further increase in impeller speed (i.e. from 250 to $350 \mathrm{rpm}$ ) resulted in lower conversion profile. Thus, the maximum conversion was achieved at $250 \mathrm{rpm}$. Conversion enhancement with an increase in the impeller speed can be anticipated as the mechanical agitation directly influences the emulsification and nucleation stages. Emulsification affects the rate of polymerization at the beginning of the reaction. At the higher impeller speeds, the mixing of the reaction mixture is improved. This enhances the probability of the reaction between reactants and thus the rate of polymerization (Fathi Roudsari et al., 2013). Besides, the initiator performance improves as the result of the improved recirculation achieved at the higher impeller speed within the reactor. For impeller speeds greater than $250 \mathrm{rpm}$, the conversion decreased. Vigorous stirring at $350 \mathrm{rpm}$ induced instability in emulsion as foaming appeared on the surface of the emulsion samples. In fact, the intensive mixing exerted an excessive shear rate on the emulsion mass. Instability in emulsion polymerization systems can occur at higher 
impeller speeds (Kemmere et al., 1998). Figure 5.1-1 (both plots) also show the polymerization profiles at 50 and $60^{\circ} \mathrm{C}$. The effect of temperature on the conversion has already been studied in literature (Scora et al., 2004). Nevertheless, we intended to obtain the results at two different temperatures to study the effect of incorporation of baffles and the impeller speed at these two temperatures to better demonstrate the impact of these factors compared to the temperature effect on the polymer properties. As expected, these data indicate that the temperature has an appreciable effect on the monomer conversion. At higher temperature, the decomposition rate of the initiator increases and thus more radicals are produced, which in turn lead to the higher monomer conversion (Scora et al., 2004).

On the other hand, as it can be observed in Figure 5.1-1, the complete conversion could not be achieved. According to Soh and Sundberg (1982), it has often been noted that polymerizations carried out at temperatures significantly below the glass transition temperature of the pure polymer do not appear to reach full conversion. Besides, the limiting conversions can be attributed to the decreased initiator efficiency and decrease in the decomposition rate of the initiator at the isothermal reaction temperature (Faldi and Tirrell, 1994). Furthermore, in our study, the monomer was not purified as it was intended to resemble the actual industrial case. Therefore, all the mentioned reasons can be attributed to the limited conversion as observed in Figure 5.1-1. Furthermore, the influence of the baffles on the conversion was studied in this research. The installation of baffles decreased the conversion about $7 \%$ at $50^{\circ} \mathrm{C}$ and $250 \mathrm{rpm}$ when the baffles were mounted as shown in Figure 5.1-1a. The reduction in conversion at $50^{\circ} \mathrm{C}$ due to the use of baffles was about $2 \%$ at $20 \mathrm{rpm}$ and $350 \mathrm{rpm}$. 
a)

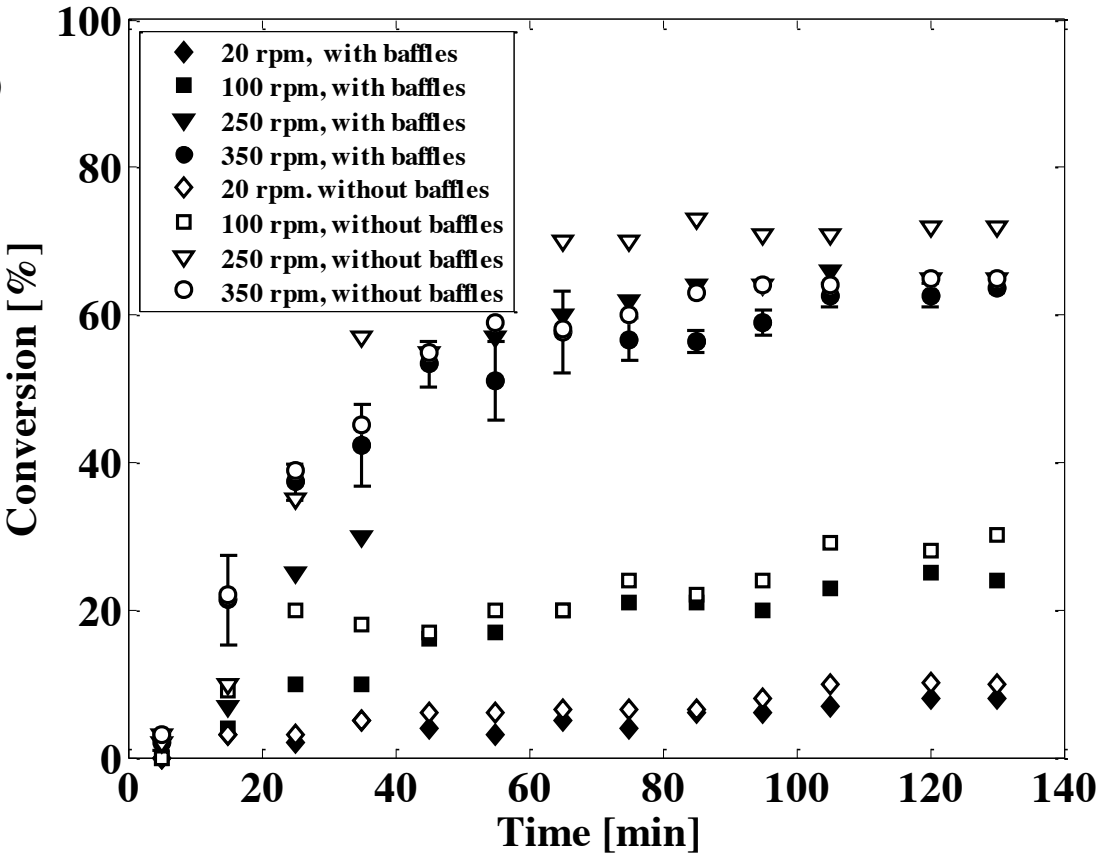

b)

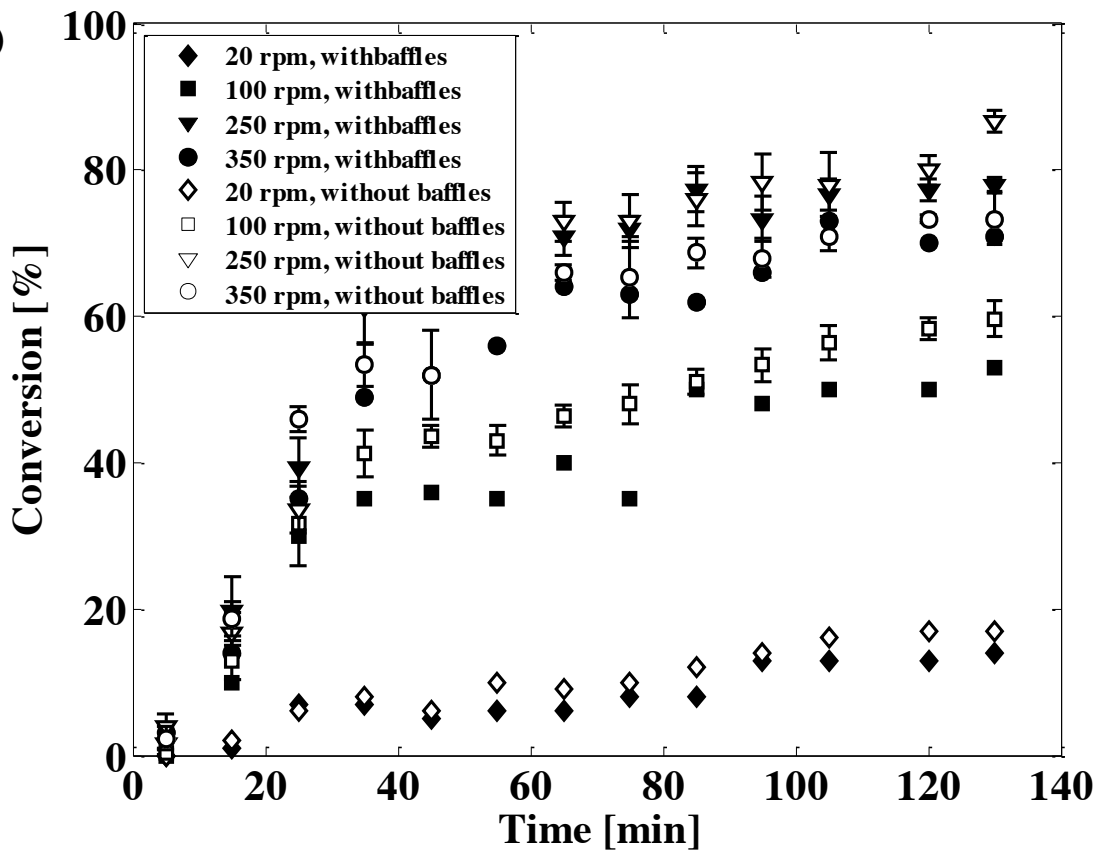

Figure 5.1-1 Conversion as a fucntion of time at the speed of 20, 100, 250 and $350 \mathrm{rpm}$, with and without baffles at the isothremal reaction temperature of a) $50^{\circ} \mathrm{C}$ and b) $60^{\circ} \mathrm{C}$. 
As depicted in Figure 5.1-1b, the use of baffles at $60^{\circ} \mathrm{C}$ decreased the conversion by about $9 \%$ at $250 \mathrm{rpm}$ and by about $2-3 \%$ at $20 \mathrm{rpm}$ and $350 \mathrm{rpm}$. At both set point temperatures, the maximum reduction in conversion due to the use of baffles was observed at $250 \mathrm{rpm}$ and the least effect was observed at $350 \mathrm{rpm}$ and $20 \mathrm{rpm}$. It means that the baffles did not have a significant effect on the conversion at very low and very high impeller speeds. In contrast, the baffles had the most impact on the conversion at the impeller speed of $250 \mathrm{rpm}$.

Generally, baffles increase the axial velocity component that promotes circulation and reduce the tangential or swirl velocity. This lower tangential velocity leads to a higher relative velocity and shear rate near the impeller. Baffling is always required for liquid-liquid dispersion, with the exception of suspension polymerization and certain highly shear-sensitive emulsion polymerizations (Paul et al., 2004). In our study, the use of baffles, increased the shear inside the reactor and as the latex was shear sensitive, the agglomeration occurred which is more notable at the impeller speed of $250 \mathrm{rpm}$ at both temperatures of 50 and $60{ }^{\circ} \mathrm{C}$ which contributed to the slight reduction in conversion. In addition, according to literature (Paul et al., 2004), the baffle placement is important in determination of the surface flow of the dispersion. The location of the top edge of the baffles relative to the liquid surface can creating eddies that are helpful in facilitating drop suspension. When baffle tips are just below the surface, unrestricted eddy motion facilitates engulfment of surface materials into the bulk liquid. If baffles extend through the surface, they create local stagnation, causing slow surface engulfment and sometimes pooling. In our experimental set up, the baffle set was extended through the surface of liquid. Therefore, the slight pooling of monomer at the surface may have reduced the monomer conversion. Besides, a minimum distance of baffles from the wall enables liquid to pass between the baffle and the wall. On the other hand, in our study, due to restriction of available space 
inside the reactor, the baffles were attached to the wall. Therefore, it is possible that the baffle surface wetted by the dispersed phase collected the monomer droplets which resulted in lower conversion.

By comparing the number of particles in Table 5.1-1, it is evident that the number of particles was larger in the reactor without baffles runs compared to the reactor with baffles experiments, which verifies the elevated conversion. Furthermore, baffles led to agglomeration of colloidal particles as will be discussed afterward in this section.

Table 5.1-1 Final particle numbers at different reactor conditions.

\begin{tabular}{|c|cccc|}
\hline $\begin{array}{c}\text { Reactor } \\
\text { Condition }\end{array}$ & $\mathbf{5 0}^{\circ} \mathbf{C}$, Baffled & $\mathbf{5 0}^{\circ} \mathbf{C}$, Unbaffled & $\mathbf{6 0}^{\circ} \mathbf{C}$, Baffled & $\mathbf{6 0}^{\circ} \mathbf{C}$, Unbaffled \\
\hline Impeller speed & \multicolumn{4}{|c}{} \\
$(\mathbf{r p m})$ & Particle Numbers in the Mixture & \\
\hline 20 & $8.78 \times 10^{15}$ & $1.09 \times 10^{16}$ & $1.6 \times 10^{16}$ & $2.3 \times 10^{16}$ \\
100 & $1.47 \times 10^{16}$ & $2.1 \times 10^{16}$ & $3.5 \times 10^{16}$ & $5.3 \times 10^{16}$ \\
250 & $3.15 \times 10^{16}$ & $3.9 \times 10^{16}$ & $4.6 \times 10^{16}$ & $6.2 \times 10^{16}$ \\
350 & $3.3 \times 10^{16}$ & $3.86 \times 10^{16}$ & $4.5 \times 10^{16}$ & $6.2 \times 10^{16}$ \\
\hline
\end{tabular}

Figure 5.1-2 shows the polymer mean nano particle size as a function of conversion at the different impeller speeds $(20,100,250$, and $350 \mathrm{rpm})$ with and without baffles at the isothermal reactor temperature of (a) $50^{\circ} \mathrm{C}$ and (b) $60^{\circ} \mathrm{C}$. 
These data demonstrate that the nano particle size increased when the impeller speed was varied from 20 to $250 \mathrm{rpm}$. In other words, the emulsification and the distribution of the initiated radicals and particles were enhanced when the efficient mixing was generated with the elevation of the impeller speeds. Regardless of which particle nucleation mechanism predominates in the particle formation process, the amount of surfactant available for stabilizing particle nuclei controls the size of population of latex particles (Hansen and Ugelstad, 1978). The emulsifier or surfactant molecules required to stabilize these primary particles come from those dissolved in the continuous aqueous phase and those adsorbed on the emulsified monomer droplet surfaces (Chern, 2006).

Generally, in emulsion polymerization, monomer can be transported to the growing latex particles by molecular diffusion from the continuous aqueous phase or by the shear induced collision between the monomer droplets and particles (Chern, 2006). Therefore, increase in the particle size with improvement in mixing from $20 \mathrm{rpm}$ to $250 \mathrm{rpm}$ can be attributed to both molecular diffusion and shear induced collision mechanisms. In the other hand, a further increase in the impeller speed (e.g. greater than $250 \mathrm{rpm}$ ) resulted in slight reduction of the nano particle diameters. As the surfactant concentration was constant in all runs at different mixing rates, the instability of emulsion at excessive stirring can only be due to collision of radicals and termination reaction inside the particles to stop the polymerization. Figure 5.1-2 (both plots) depict the thermal effect during the polymerization on the particle size profile. Larger nano particle sizes were obtained at $50^{\circ} \mathrm{C}$ while the baffles were installed.

Raising the reaction temperature from $50^{\circ} \mathrm{C}$ to $60^{\circ} \mathrm{C}$ (Figure $5.1-2 \mathrm{~b}$ ) had an adverse effect on the particle sizes despite an elevation in conversion. 
a)

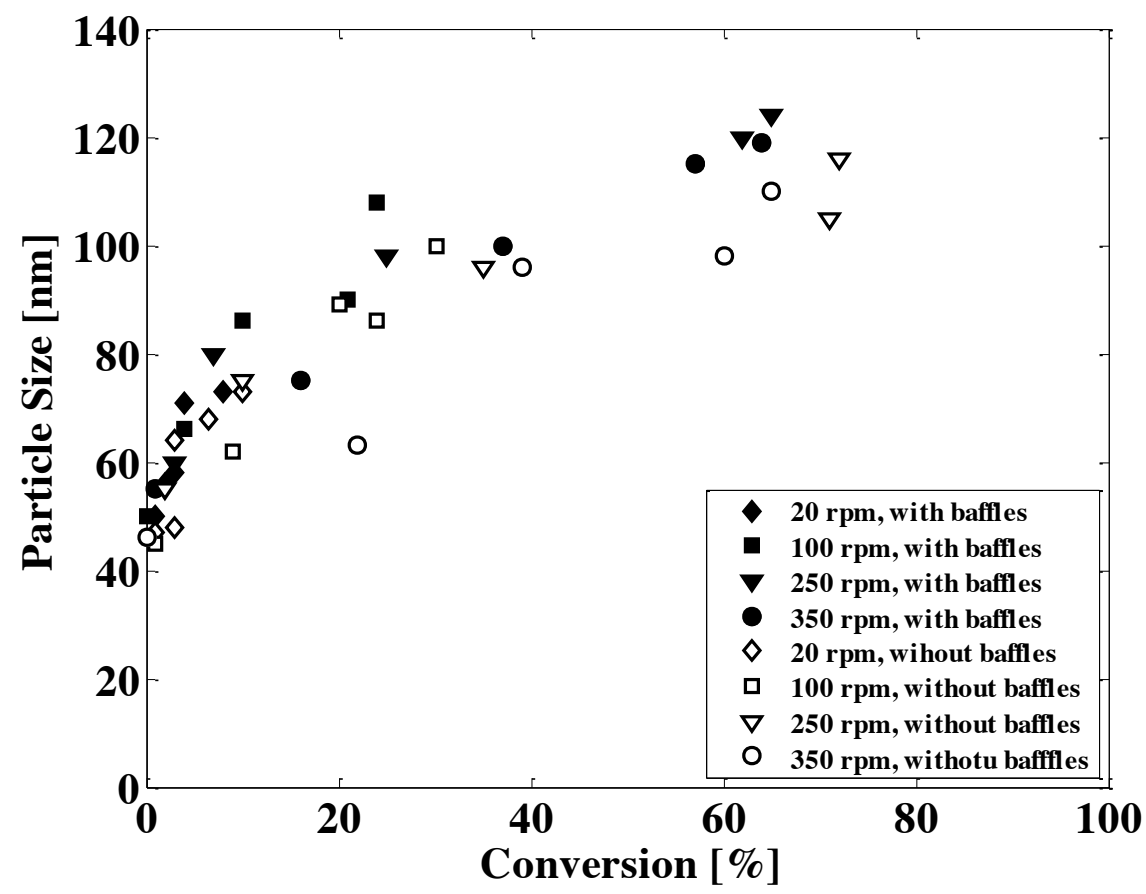

b)

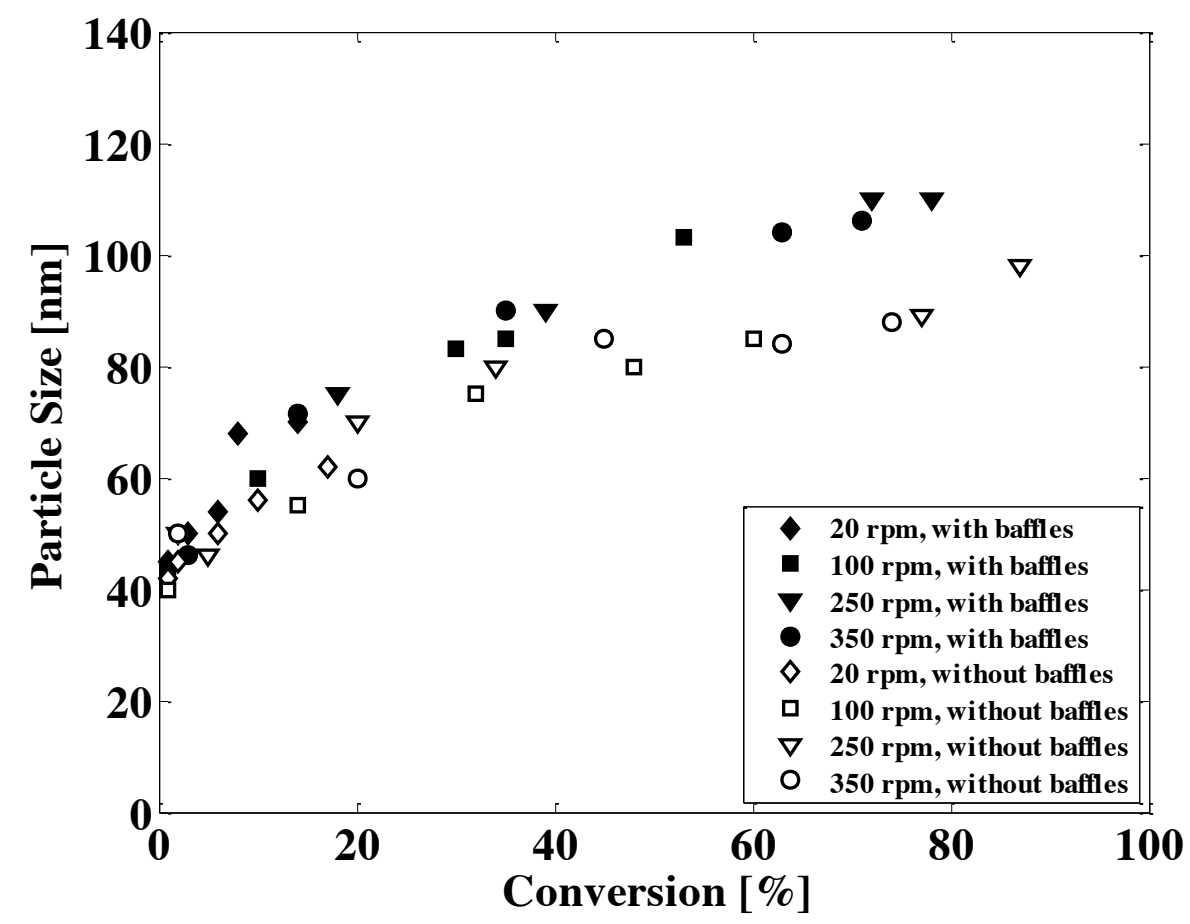

Figure 5.1-2 Mean particle size as a fucntion of time conversion at the speed of 20, 100, 250 and $350 \mathrm{rpm}$, with and without baffles at the isothremal reaction temperature of a) $50^{\circ} \mathrm{C}$ and b) $60^{\circ} \mathrm{C}$. 
The reduction of the nano particle size with an increase in temperature has also been reported in literature (Fathi Roudsari et al., 2013, Faldi and Tirrell, 1994, Hansen and Ugelstad, 1978, Hansen and Ugelstad, 1979). The overall rate of polymerization increases with an increase in temperature. Temperature increases the rate by increasing both propagation rate constant and number of particles. The increase in the number of particles is due to the increased rate of radical generation at higher temperatures (Table 5.1-1). Opposing this trend is the small decrease in the concentration of monomer in the particles at higher temperatures. Hence, as it can be seen from Figure 5.1-2, the particle size was reduced at the elevated temperature. Figure 5.1-2 (both plots) also shows the influence of the baffles on the particle size at the two temperatures, $50^{\circ} \mathrm{C}$ and $60^{\circ} \mathrm{C}$. These data indicate that by removing the baffles, the particle size became smaller. Therefore, the presence of baffles enhanced the shear flow and consequently slightly larger polymer particles were produced due to agglomeration. Another assumption is that removing the baffles resulted in the vortex flow and the growth of the particles slowed down due to the swirling and solid flow. It may be postulated that the monomer had less chance to be absorbed into the micelles or radical oligomers in the aqueous phase since the monomer transportation was limited due to vortex flow. Overall, it can be concluded that the largest particle size profile as a function of conversion was achieved at the lower temperature in a reactor equipped with the baffles.

It has been reported (Ellis and Taylor, 1994) that during the course of isothermal polymerization (without monomer addition), the molecular weight decreases mildly due to the monomer depletion. Besides, according to some researchers (Cunningham et al., 2000), higher initial oxygen levels in the vapor space reduced the molecular weight and led to smaller latex particles. In this research, the influence of the impeller speed, reactor temperature, and the presence of 
baffles on the average molecular weight as a function of conversion is plotted in Figure 5.1-3. Samples were taken at time intervals of 25,75 , and $130 \mathrm{~min}$ after the injection of the initiator into the reactor. As can be discerned in Figure 5.1-3 (both plots), the weight average molecular weight increased with a rise in the impeller rotational speed from 20 to $350 \mathrm{rpm}$ for the first sample taken 25 minutes after the initiator injection.

For the second and third samples taken from the reactor after 75 and 130 min, the increase in the weight average molecular weight was observed with an increase in the impeller speed up to 250 rpm. However, further increase in the rotational speed, eventuated in a decrease in the molecular weight. Again, the interpretation is analogous to the particle size results as discussed for Figure 5.1-2 and shows that better recirculation of the reaction mixture at higher agitation rates can improve the initiated radical distribution throughout the reactor, which led to an enhancement of the particle growth and therefore the molecular weight. In contrast, the instability of emulsion at vigorous stirring slowed down the growth of polymer particles at higher stages of polymerization reaction with lower molecular weights. Furthermore, an increase in the reaction temperature from $50^{\circ} \mathrm{C}$ to $60^{\circ} \mathrm{C}$ resulted in a decrease in the average molecular weight of the sample polymers as presented in Figure 5.1-3. As mentioned before, at higher temperature, the rate of propagation rate and number of particles are enhanced. In the other hand, it is likely that the transfer to monomer and polymer was increased due to the higher movement of chains inside the particles, and the depletion of monomer and growing radicals was also enhanced under the thermal effect. These phenomena might contribute to the reduction of the molecular weight.

Profiles of the average molecular weight in the reactors with and without baffles are also depicted in Figure 5.1-3. 
a)

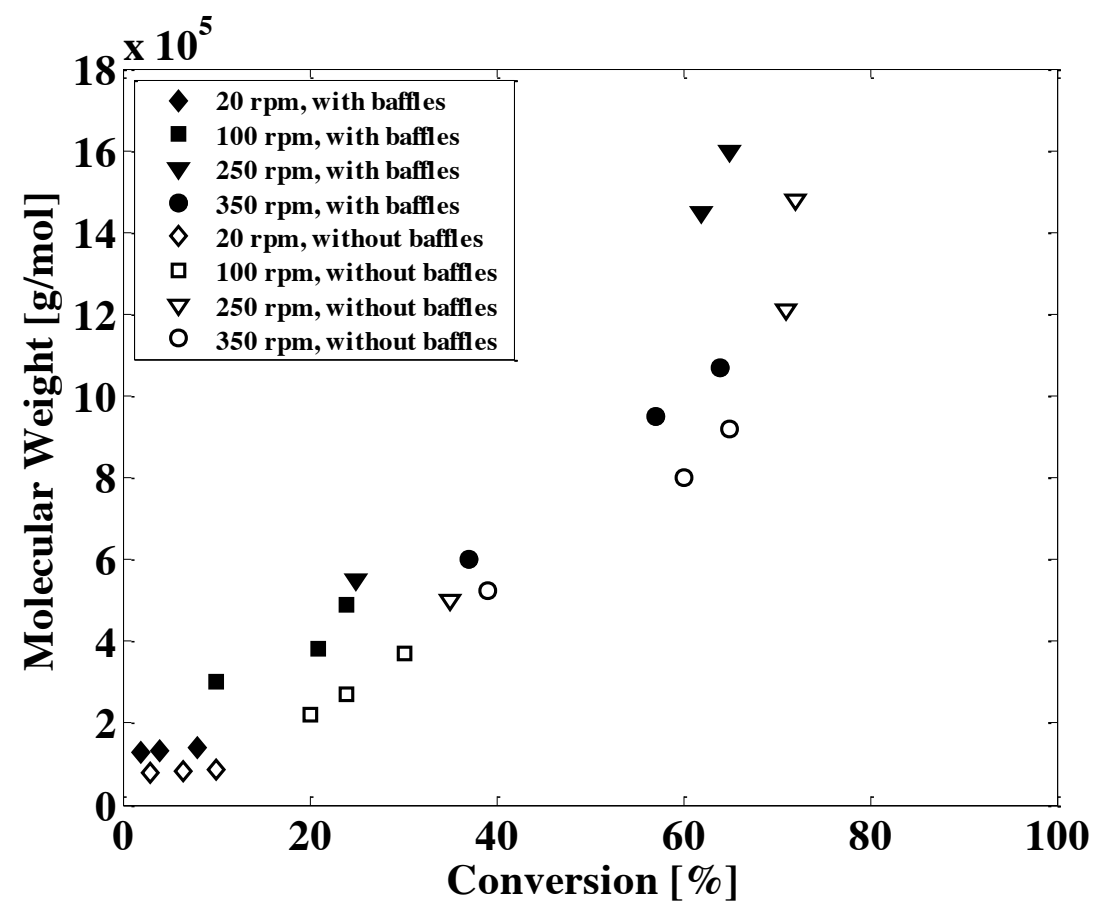

b)

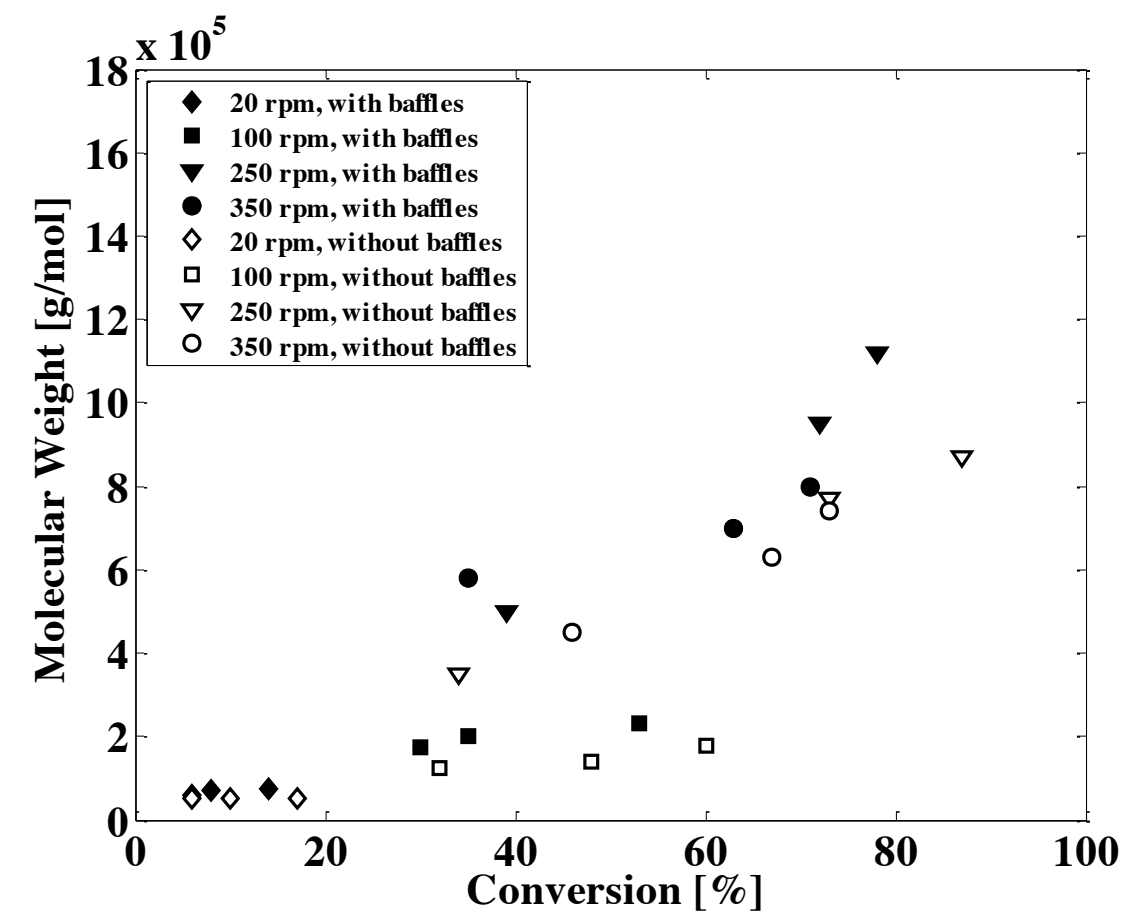

Figure 5.1-3 Average weight molecular weight as a fucntion of time conversion at the speed of $20,100,250$ and $350 \mathrm{rpm}$, with and without baffles at the isothremal reaction temperature of a) $50^{\circ} \mathrm{C}$ and b) $60^{\circ} \mathrm{C}$. 
It can be observed that the installation of the baffles resulted in the higher average molecular weights. As mentioned earlier, the use of the baffles eliminated the formation of the vortex in the reactor and converted it into an axial flow. From this data trend of polymer product with higher molecular weights we can postulate that when the vortex flow is eliminated in the reactor vessel, there is a higher probability of the monomer absorption into the micelles or into insoluble radical chains in the aqueous phase. According to literature (Mendoza Marin et al., 2006), the influence of baffles on the molecular weight distribution in an emulsion polymerization reactor equipped with the internal angular baffles was also investigated and the results showed that the use of baffles had only a marginal effect on the molecular weight distribution (Mendoza Marin et al., 2006). Overall, the highest average molecular weight was achieved in the reactor with baffles at the reaction temperature of $50^{\circ} \mathrm{C}$. Figure 5.1-4 shows plots of the polydispersity of the polymer samples obtained in this study. These data show that the polydispersity increased with conversion. This trend was due to the growth of the polymer particles, which resulted in the production of the larger particles with time. Polydispersity of a polymer increases with the conversion as a consequence of chain branching reactions, transfer to polymer, and terminal double bond polymerization, which becomes more important as the polymer concentration increases (Asua, 2007). This explanation can be applied to our study as well. The only exception was observed in Figure 5.1-4a (100 rpm, without baffles), in which the second sample had a slightly higher PDI than the next sample (from 2.4 to 2.3), which was attributed to the sample preparation issues for the GPC analysis. A comparison between the data shown in Figure 5.1-4 (both plots) reveals that the use of baffles resulted in a narrower molecular weight distribution. It can be implied that the molecular weight was better controlled by the use 
a)

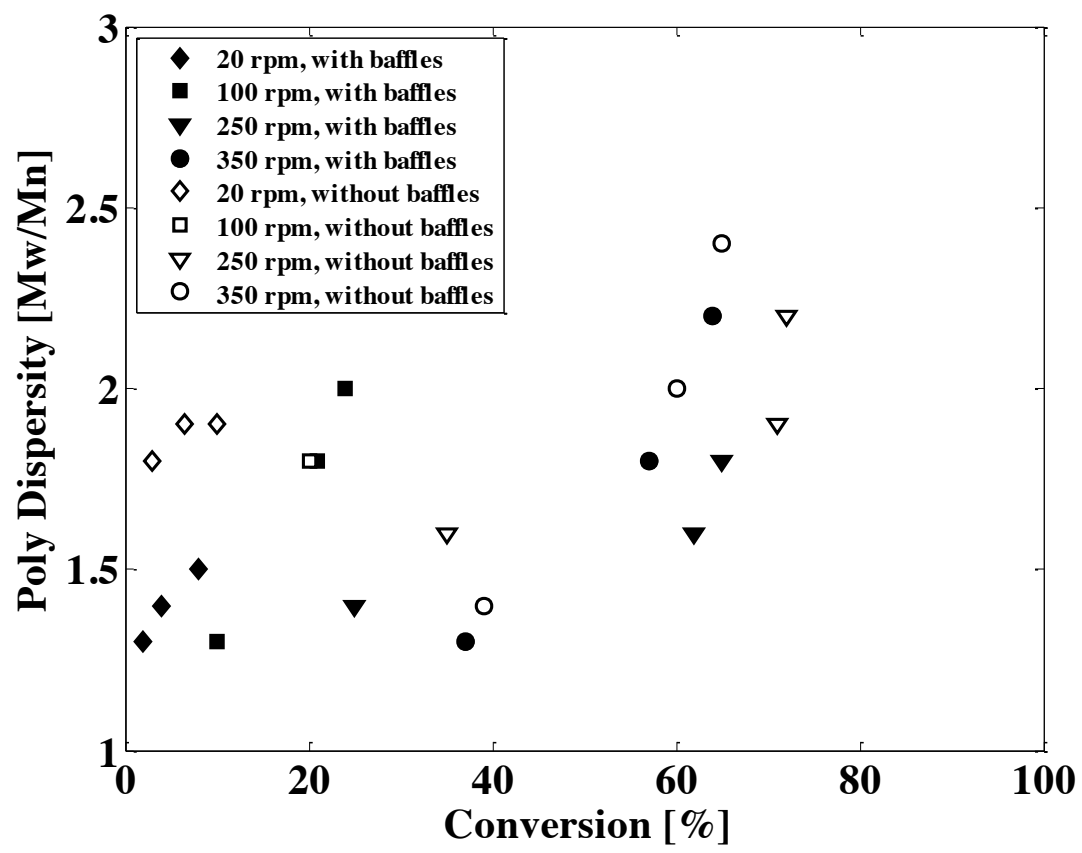

b)

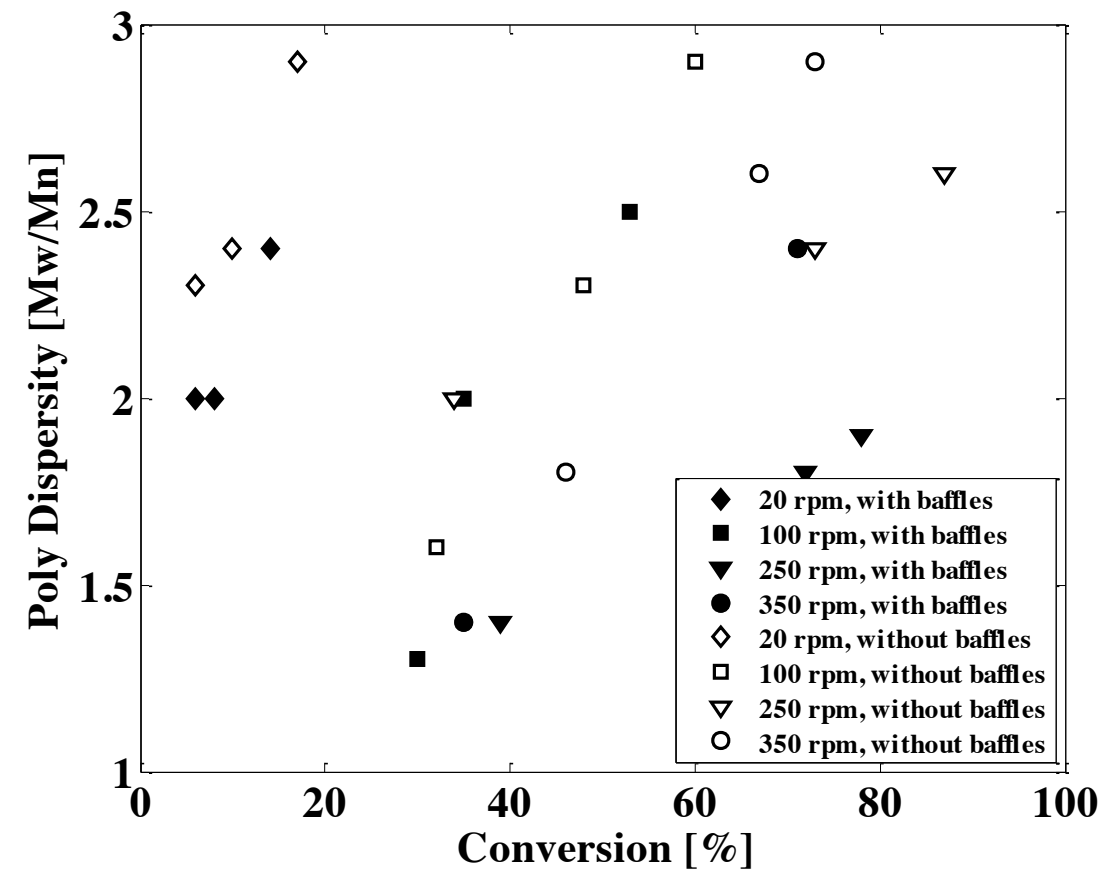

Figure 5.1-4 Polydispersity of polymers as a fucntion of time conversion at the speed of 20, 100,250 and $350 \mathrm{rpm}$, with and without baffles at the isothremal reaction temperature of a) $50^{\circ} \mathrm{C}$ and b) $60^{\circ} \mathrm{C}$. 
of the baffles. An increase in the temperature did not show a significant effect on PDI at the early stage of polymerization, but contributed to the higher polydispersity as the conversion was enhanced.

In Figure 5.1-5, the conversion, mean particle size, and average molecular weight as a function of the impeller speed are separately plotted after $130 \mathrm{~min}$ for the reactors with and without baffles at $60^{\circ} \mathrm{C}$. In Figure 5.1-5 (a, b and $\mathrm{c}$ ), the conversion, mean particle size, and average molecular weight of the final latex samples were enhanced with an increase in the impeller speed due to better recirculation and better chance of reaction and emulsification. However, further raise in the agitation rate (greater than $250 \mathrm{rpm}$ ) resulted in instability of emulsion and lowered all the mentioned polymer qualities. Use of baffles in the reactor resulted in excessive shear flow and stagnation of monomer droplets on the surface of the reaction mixture. Furthermore, the wetted surface of baffles collected the monomer droplets and therefore the monomer conversion slightly decreased. Besides, installation of baffles in our experiment led to agglomeration of particles and increase in the mean particle size (Figure 5.1-5b). The average molecular weights as shown in Figure 5.1-5c had a slight decrease when the baffles were removed. We can assume that the swirling flow in the reactor without baffles limited the monomer transport to the growing particles and thus the molecular weight decreased.

The volumetric size distribution of particles at the impeller speed of $250 \mathrm{rpm}$ and the reaction temperature of $60^{\circ} \mathrm{C}$ in the reactors with and without baffles is plotted in Figure 5.1-6. Three samples were taken at 25, 75, and $130 \mathrm{~min}$ after the initiator was injected into the reactor. The size distribution of the particles produced in both reactors with and without baffles drifted distinctly to the broader profiles as the reaction time progressed from 25 to $130 \mathrm{~min}$. 
a)

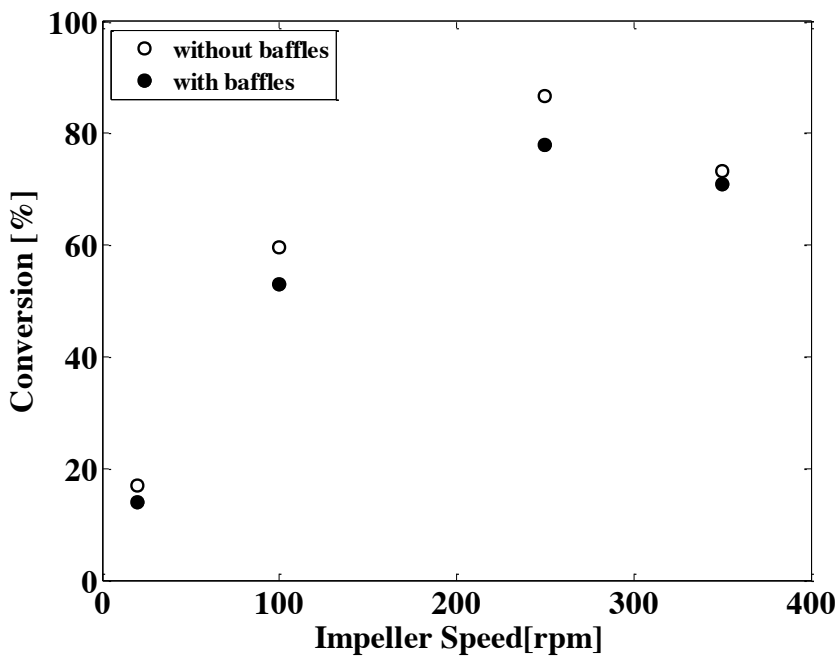

b)

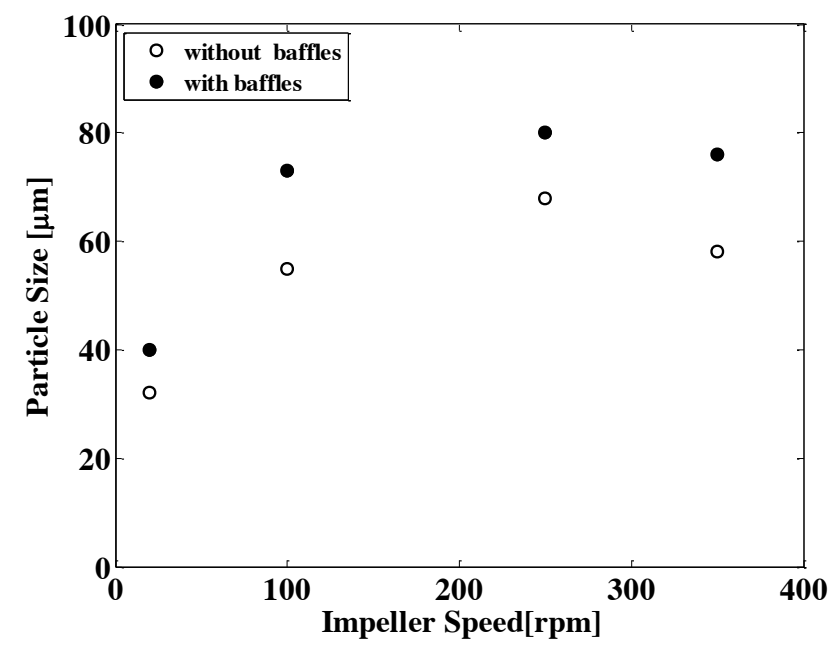

c)

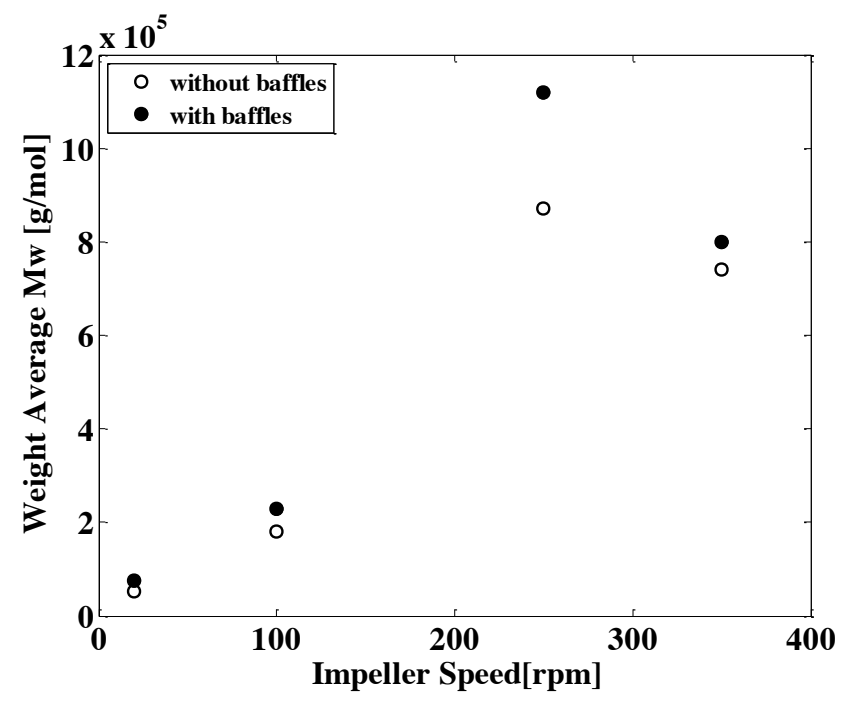

Figure 5.1-5 Effect of impeller speed on a) conversion, b) mean particle size and c) average weight molecular weight after $130 \mathrm{~min}$. 
Besides, the effect of the baffle is clearly observed in this Figure as at each specified reaction time, the distribution curve measured for the reactor without baffles was wider than that for the reactor with baffles.

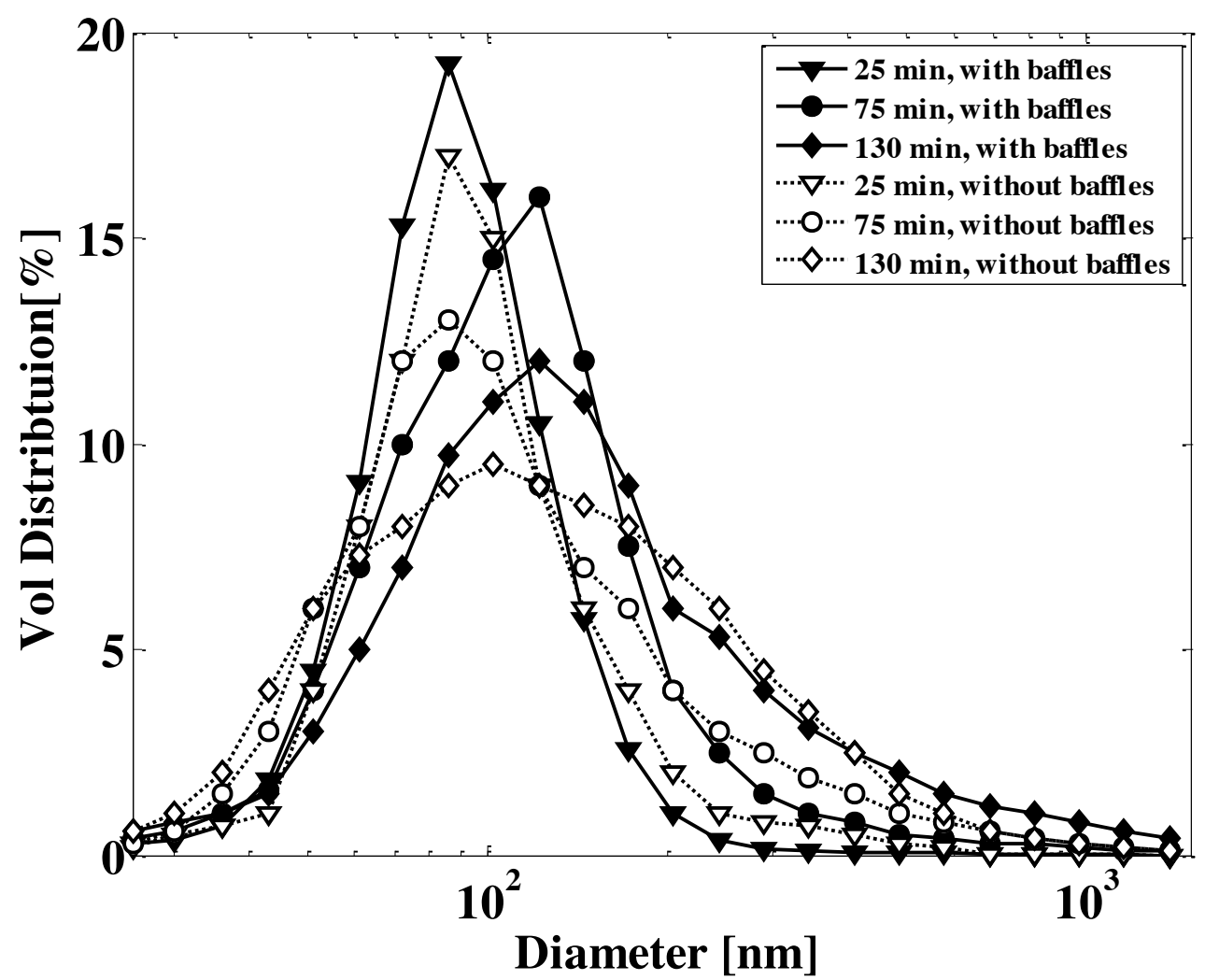

Figure 5.1-6 Particle size distribution at $60^{\circ} \mathrm{C}$ and the impeller speed of $250 \mathrm{rpm}$, reactors with and without baffles.

The particle size distribution is a consequence of the distribution of times at which different polymer particles are nucleated. An explanation for a broader distribution with an increase in the reaction time is proposed as follows. When particles are first formed at the initial stage, the distribution is normal. The free radicals formed in the aqueous phase diffuse into the primary particles and the majority of them escape into water again. There is a higher chance that a particle with a larger volume gets more radicals than a small one. Thus, a large particle will 
propagate at a faster rate to form a larger particle. During stage II of the polymerization reaction, more polymer primary particles are formed by micellar and homogeneous nucleation. This increases the population of the small particles while the large particles receive more radicals and propagate at a rapid rate (Chern, 2006). This is an explanation as why the particle size distribution is broad in a conventional emulsion polymerization. Despite the occurrence of excessive shear flow in our experiments due to use of baffles as already discussed, the vortex formation was minimized in the reactor with baffles, and therefore the possibility of having a more uniform reaction mixture increased. Thus, the size distribution of the particles yielded in the reactor with baffles was narrower than that produced in the reactor without baffles during the polymerization process.

Figure 5.1-7 shows the refractive index (RI) response peaks extracted from GPC data for 250 $\mathrm{rpm}$ in the reactor with baffles operated at $60^{\circ} \mathrm{C}$. We showed the RI signal as a function of elution volume (Mertoğlu, 2004) to confirm the change of molecular weight with increase in reaction time and conversion. The different molecular species are eluted from the GPC column in order of their molecular size (Rudin and Choi, 2012). Therefore, the larger molecules leave the column earlier than the smaller molecules. In Figure 5.1-7, the size exclusion chromatography traces of the polymer samples in THF exhibit the shift towards lower elution volumes with the increase in reaction time from $25 \mathrm{~min}$ to 75 and $130 \mathrm{~min}$, with conversion of $39 \%$, $72 \%$, and $79 \%$ respectively. This approves higher molecular weight due to growth of polymer particles in the reactor. Besides, the well-resolved unimodal peaks in the GPC chromatograms shows a uniform distribution in the molecular weight of polymer particles.

Finally, Figure 5.1-8 demonstrates the scanning electron microscopy (SEM) images (Hitachi S2150) of the dried end product latex at different impeller speeds of 100, 250, and $350 \mathrm{rpm}$. 


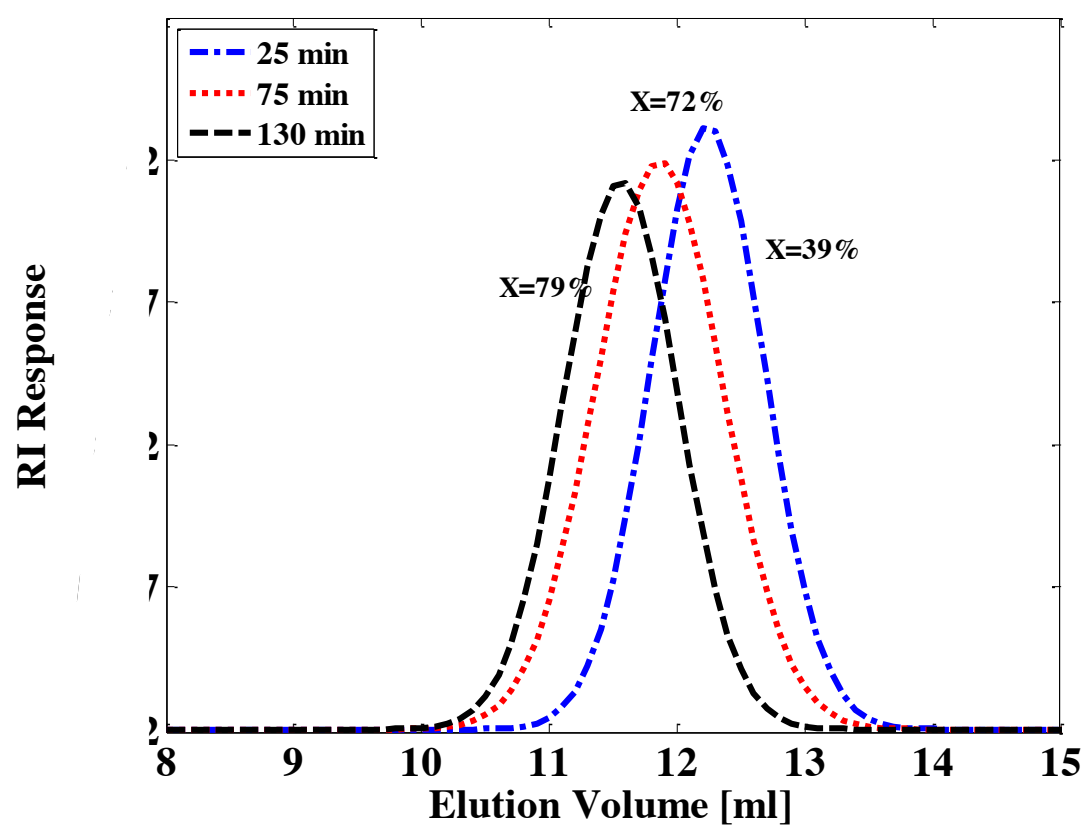

Figure 5.1-7 RI response of GPC analysis at $60^{\circ} \mathrm{C}$, reactor with baffles and the impeller speed of $250 \mathrm{rpm}$.

The reaction temperature was $60^{\circ} \mathrm{C}$ and the reactor was equipped with the baffles. These images show that the dried particle size increased when the agitation speed was varied from $100 \mathrm{rpm}$ to $250 \mathrm{rpm}$. However, the particle sizes decreased slightly at $350 \mathrm{rpm}$. Besides, the polymer particles had more uniform shape and size distributions at the higher stirring rates (especially at $250 \mathrm{rpm})$. The information obtained in this study can improve the existing emulsion polymerization processes and has the potential to contribute to the development of the novel polymerization processes.

\subsubsection{Concluding Remarks}

Emulsion polymerization of MMA was carried out in a 2-liter reactor equipment with a pitched blade turbine. The effects of the impeller speed, baffles, and temperature on the conversion, 
a)

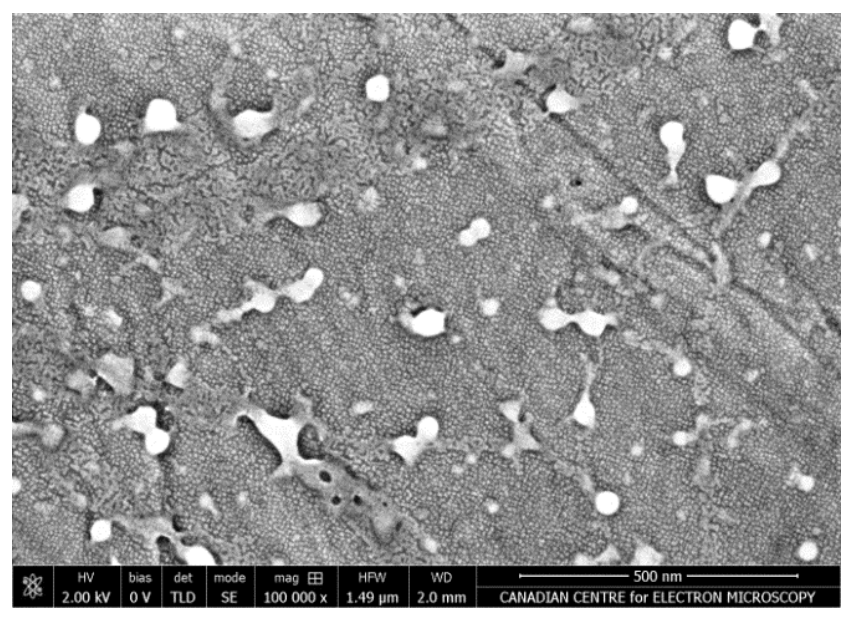

b)

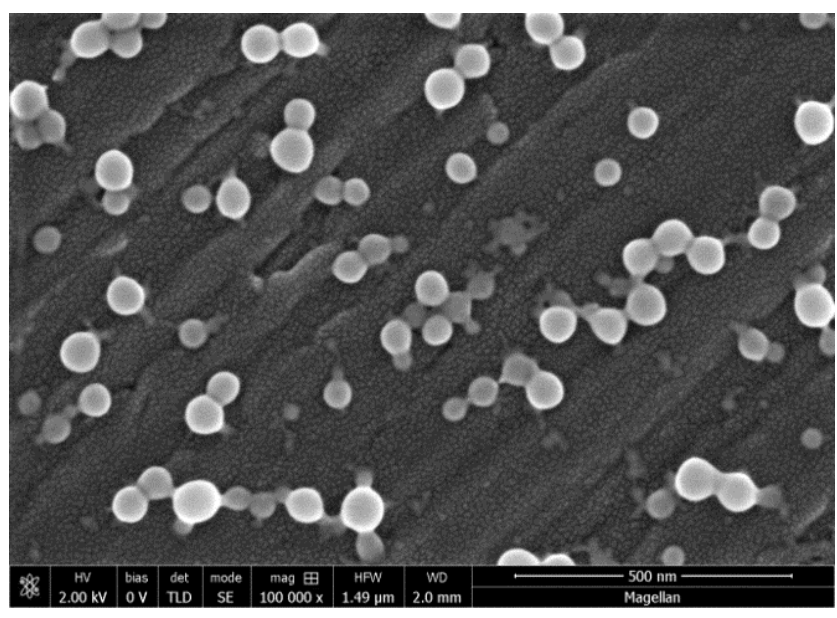

c)

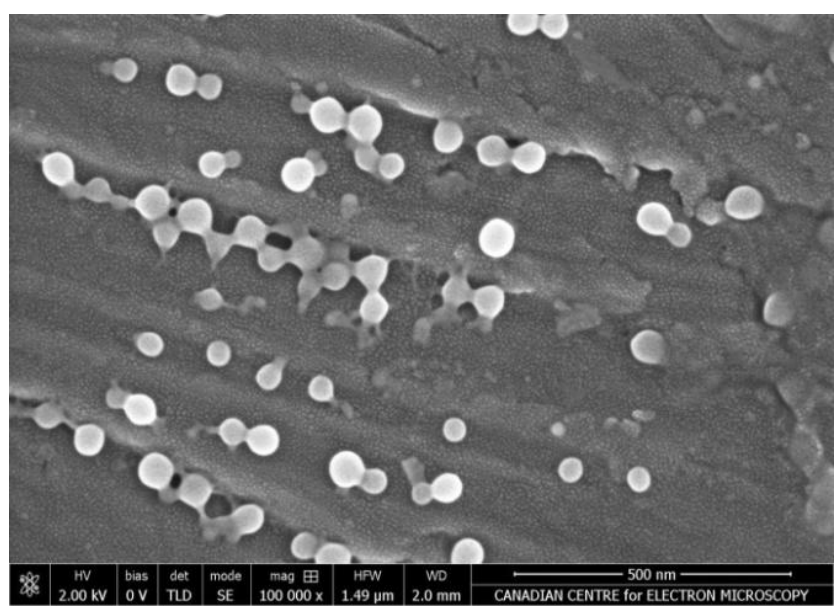

Figure 5.1-8 Scan Electron Microscopy images at $60^{\circ} \mathrm{C}$, reactor with baffles and the impeller speed of a) $100 \mathrm{rpm}$, b) $250 \mathrm{rpm}$ and c) $350 \mathrm{rpm}$. 
molecular weight, and particle size were investigated. It was found that the monomer conversion, particle size, and molecular weight were increased when the impeller speed was raised from 20 to $250 \mathrm{rpm}$. However, further increase in the rotational speed (from 250 to $350 \mathrm{rpm}$ ) resulted in the reduction of the monomer conversion, particle size, and molecular weight of the polymer. Installation of the baffles decreased the monomer conversion particularly at $250 \mathrm{rpm}$, but the particle size and the molecular weight of the polymer produced in the reactor with baffles were slightly enhanced. Besides, the number of particles was higher for the reactor without baffles. However, installation of baffles led to the formation of the polymer with a narrower size distribution. While an increase in the reaction temperature from $50^{\circ} \mathrm{C}$ to $60^{\circ} \mathrm{C}$ had an adverse effect on the polymer particle sizes and molecular weights, the conversion of the monomer was improved at the higher temperature. Also, the number of particles was higher at $60^{\circ} \mathrm{C}$ compared to that at $50^{\circ} \mathrm{C}$. The highest values for the average particle size and molecular weight were observed at $50^{\circ} \mathrm{C}$ and the reactor with baffles.

An increase in the temperature did not result in the variation of PDI at the early stage of polymerization, but contributed to the higher PDI as the conversion enhanced. SEM images demonstrated that the polymer particles had more uniform shape and size distributions at the higher stirring rates (250 and $350 \mathrm{rpm})$ and the largest particle sizes were produced at $250 \mathrm{rpm}$. The finding of this study can be applied to improve the reactor design, to optimize the polymerization conditions, and to adjust the end product quality with the minor likeliness of adding the so-called reactive agents, which would be definitely reduces the environmental impact and the production cost. In the work, the effects of other mixing parameters such as the impeller type and the number of impellers are discussed in detail. 


\subsection{Impact of impeller type on MMA emulsion polymerization in a batch reactor (Fathi Roudsari et al., 2014b)}

\subsubsection{Introduction}

In emulsion polymerization reactors, it is difficult to reproduce uniform mixing similar to that in small reactors. Hence, non-uniform mixing is a common source of variability in particle nucleation and particle size distribution. The size distribution influences the molecular weight distribution and polymer architecture, and consequently latex properties. For the progress in future commercial latex products, it is essential to develop a cost efficient method to control the polymer colloid properties as well as the polymer chemistry research. This study thoroughly discusses the effects of the stirring rate, type of impeller and single/double impellers on the monomer conversion, mean particle size, average molecular weight, and glass transition temperature. The experimental set up is a 2L PARR reactor as shown in Chapter 3 (Figure 3.1-1) and the experimental procedure are described in Section (3.2). The pitched blade and Rushton impellers (single and double) were utilized and the experimental design is shown in Table 3.4-3.

\subsubsection{Results and discussion}

Generally, the emulsification and nucleation govern the course of the process at the beginning of the polymerization. The nucleation mechanism can be both micellar and homogenous nucleation for the partially soluble monomers (Hansen and Ugelstad, 1978; 1979a; 1979b; 1979c). The MMA solubility in water is known to be $1.5 \mathrm{~g} / 100 \mathrm{~mL}$ (Asua, 2007). Hence, under the polymerization conditions (i.e. concentration of the initiator and the emulsifier) employed in this 
study, the micellar mechanism prevailed and the homogeneous nucleation mechanism was negligible.

Besides, the rate of polymerization is mainly due to the intrinsic reaction rates involved and the transport of monomer to the growing particles (Ohmura et al., 2005). The experiments were performed from low to high agitation rates at a fixed chemical recipe at isothermal reactor temperature, i.e. $60^{\circ} \mathrm{C}$ and baffles were installed in the reactor in all experiments. Sampling was started 5 min after adding initiator at $5^{\circ} \mathrm{C}$ below the set point and a total of 13 samples were taken for each run. The monomer conversion profiles versus time at different impeller speeds are shown for the single and double pitched-blade (Figure 5.2-1a) and Rushton (Figure 5.2-1b) impellers.

In Figure 5.2-1a, the conversion was low at the impeller speed of $100 \mathrm{rpm}$ with the use of single pitched impeller. On increasing the stirring rate to $250 \mathrm{rpm}$, the conversion reached its maximum value and further increase in agitation led to a reduction in the monomer conversion. The axial flow impellers produce a constant pumping action toward the bottom of the tank followed by circulation to the top and a relatively rapid return to the impeller zone (Holland and Chapman, 1966; Spicer et al., 1996). The short circulation time produced by the axial flow impeller increases the frequency of exposure to the high intensity shear in the impeller zone, where turbulent energy dissipation rates are much larger than in the bulk zone (Spicer et al., 1996; Kim and Glasgow, 1987). The turbulent energy up to the speed of $250 \mathrm{rpm}$ produced the optimum collision of reactants and homogeneity of bulk flow to reach to the maximum conversion. 
a)

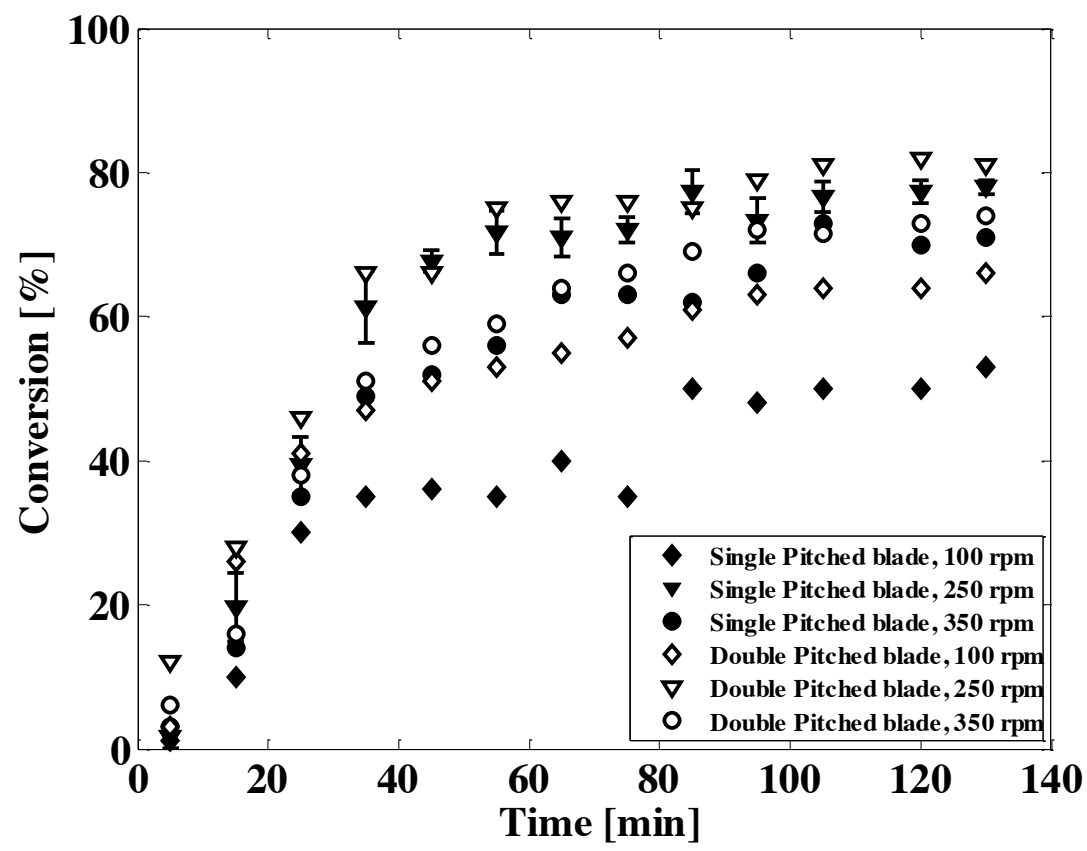

b)

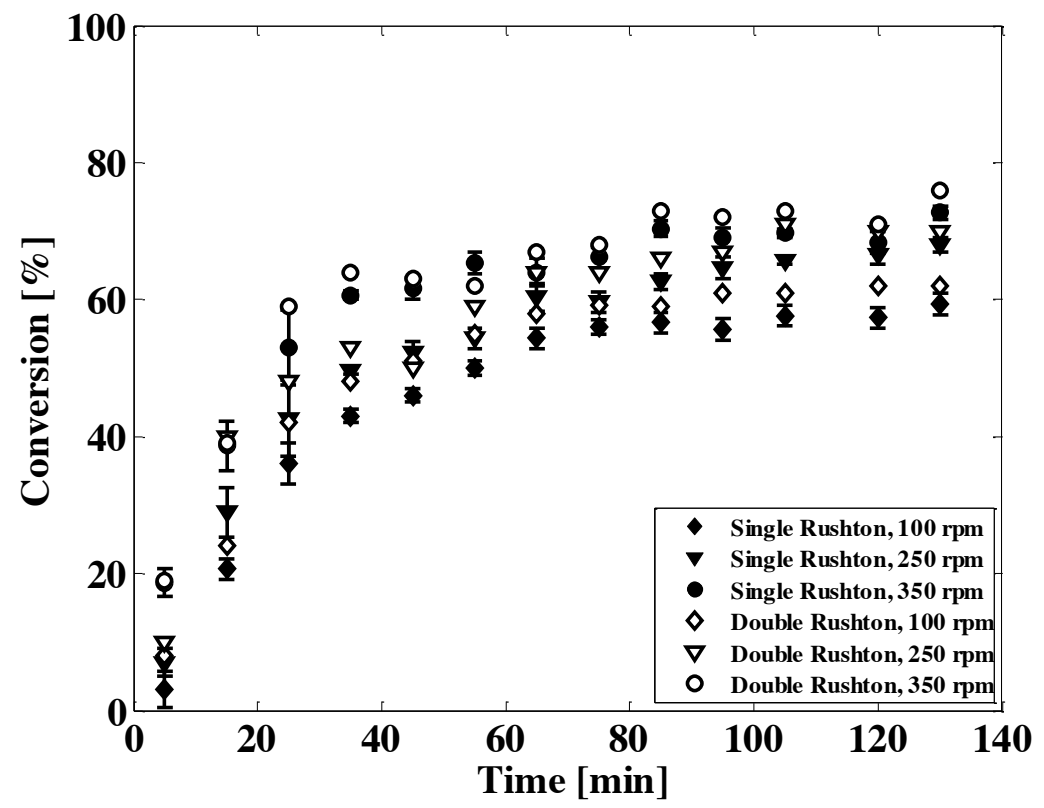

Figure 5.2-1 Monomer conversion versus reaction time for a) pitched blade turbine and b) Rushton impeller at the speeds of 100-350 rpm using both single and double impellers. 
However, further increase in the impeller speed to $350 \mathrm{rpm}$ caused a reduction in monomer conversion which can be due to the formation of vortices, instability and dead zones in the bulk mixture. Furthermore, in Figure 5.2-1a, the monomer conversion clearly increased at low impeller speed of $100 \mathrm{rpm}$ when two pitched-blade impellers were employed. The conversion increase due to the use of double pitched-blade impellers compared to single one was less significant at higher impeller speeds of $250 \mathrm{rpm}$ and $350 \mathrm{rpm}$.

In general, multiple impellers are often employed to improve circulation and narrow the distribution of shear and energy dissipation by forming more circulation loops within the tank mixture (Paul et al., 2004). Therefore, more circulation loops at the impeller speed of $100 \mathrm{rpm}$ helped form better homogeneity of the reaction mixture and improved nucleation mechanism and growth process and thus the conversion was increased.

At higher impeller speeds of 250-350 rpm, the conversion enhancement with installation of two pitched-blade impellers was not very significant. Therefore, it can be concluded that the pumping action produced by single pitched-blade were strong enough to produce bulk homogeneity through the reaction mixture and use of double pitched-blade impellers only resulted in insignificant increment in conversion. Therefore, the limiting conversion even with the use of double pitched impeller should be sought in other factors which will be shortly discussed here.

In Figure 5.2-1b, the monomer conversion at low impeller speed of $100 \mathrm{rpm}$ was higher with the use of Rushton impeller compared to the pitched-blade turbine. Using Rushton impeller was more efficient at low impeller speed of $100 \mathrm{rpm}$. The Rushton impeller creates fluid flow directed radially outward of the impeller and produces two circulating loops one below and one above the impeller. Mixing occurs between the two loops but less intensely within each loop. With increase in the Rushton impeller speed the conversion was enhanced at $250 \mathrm{rpm}$ and 350 
rpm. However, the increase in conversion was not very significant compared to the runs with the pitched-blade impeller. The radial fluid flow produced by the Rushton impeller mostly circulates into the region above the impeller. These recirculated fluid mixture then slowly return to the impeller zone. In contrast, the axial flow impeller produces a strong pumping action and a relatively rapid return to the impeller zone. Therefore, increase in the Rushton impeller speed majorly produced more shear near the impeller region and the conversion elevation can be attributed to this region at low impeller speed of $100 \mathrm{rpm}$. However, less mixture homogeneity was achieved by Rushton impeller due to insufficient pumping action. Besides, the use of double Rushton impellers as shown in Figure 5.2-1b was not so effective. By employing two Rushton impellers, more circulating loops are produced while mixing majorly occurs between the loops (Paul et al., 2004). Therefore, an enhancement of the monomer conversion was not very significant when using two Rushton impellers at different speeds. It must be mentioned that the computational fluid dynamics (CFD) analysis of the current experimental work was being conducted in the research lab to elucidate the homogeneity, turbulence and flow pattern throughout the reactor vessel.

Figure 5.2-2 depicts the monomer conversion of the samples versus impeller speed for single and double pitched-blade and Rushton impellers, taken at the end of reaction time of 130 minutes. The variation of conversion with speed was more observed for the runs using the pitched-blade turbine rather than the Rushton impeller. Moreover, installation of two pitched-blade improved the conversion at low impeller speed of $100 \mathrm{rpm}$. The complete conversion could not be achieved in the experiments. It has often been noted (Soh and Sundberg, 1982) that polymerizations carried out at temperatures significantly below the glass transition temperature of the pure polymer do not appear to reach full conversion. Besides, the limiting conversions can be 
attributed to the decreased initiator efficiency and decrease in the decomposition rate of the initiator at the isothermal reaction temperature (Faldi and Tirrell, 1994). Furthermore, in our study, the monomer was not purified as it was intended to resemble the actual industrial case. Therefore, all the mentioned reasons can be attributed to the limited conversion as observed in Figure 5.2-2.

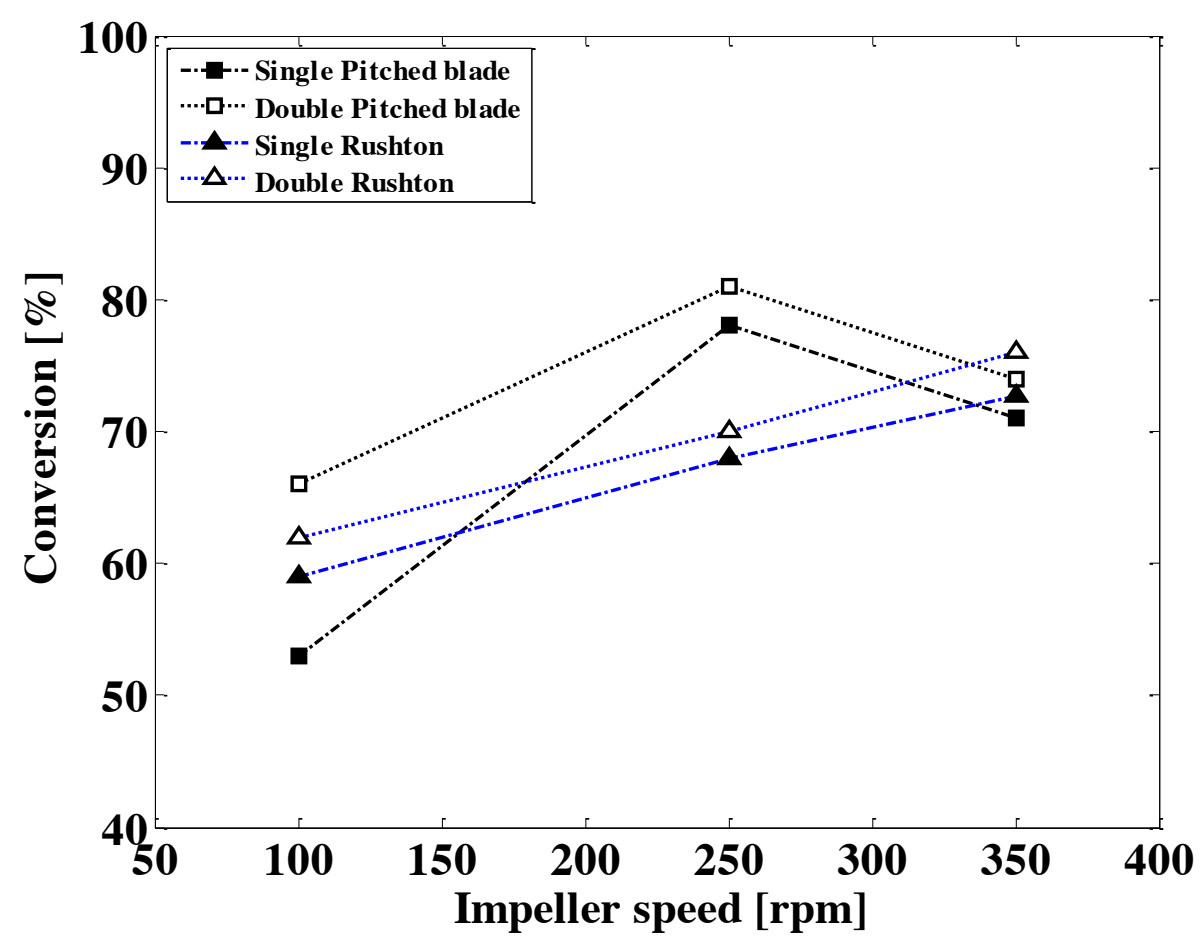

Figure 5.2-2 Final monomer conversion versus impeller speed at reaction time of $130 \mathrm{~min}$ for single/double pitched blade and Rushton impellers.

The weight average molecular weight versus conversion at different impeller speeds are plotted for the single and double pitched-blade (Figure 5.2-3a) and Rushton (Figure 5.2-3b) impellers. The samples were taken at reaction times of 25, 75 and $130 \mathrm{~min}$. 
a)

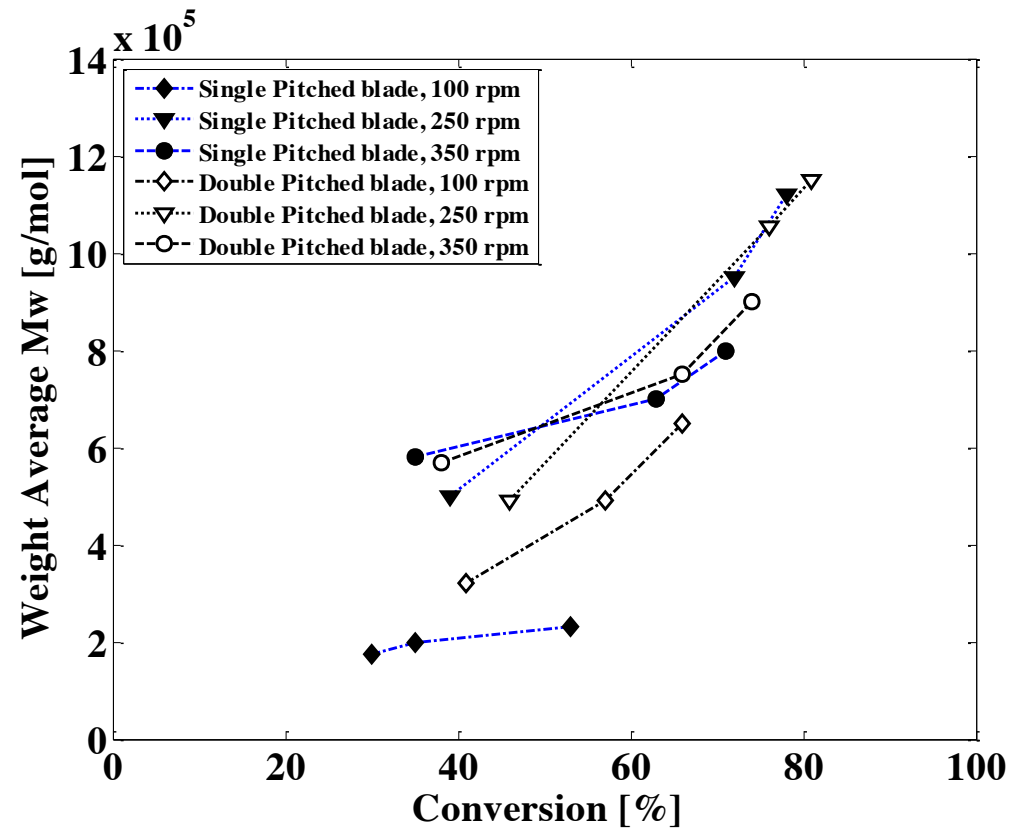

b)

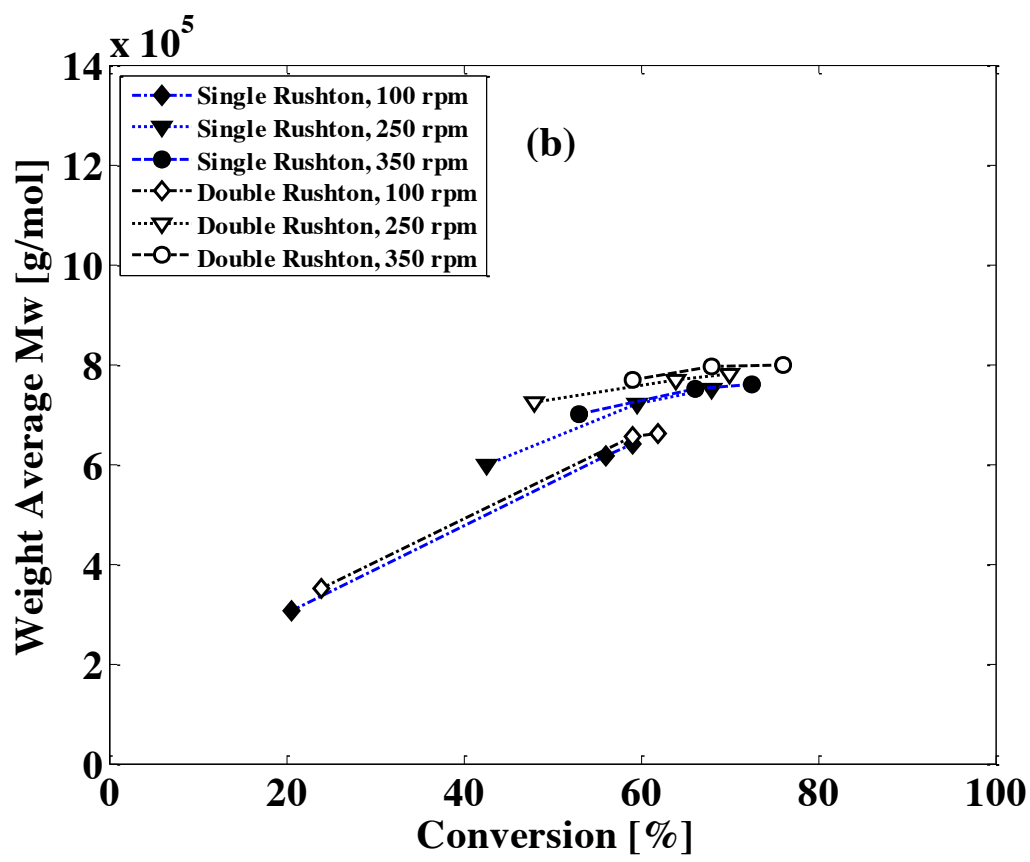

Figure 5.2-3 Weight average molecular weight versus monomer conversion for a) pitched blade turbine and b) Rushton impeller at the speeds of 100-350 rpm using both single and double impellers. 
In Figure 5.2-3a, at low impeller speed of $100 \mathrm{rpm}$, the weight average molecular weight was low. It means that due to low pumping action of the pitched-blade impeller at low speed, the emulsification was not sufficient and therefore the polymerization reaction was not significant. When the stirring rate was raised, the maximum molecular weight $\left(\mathrm{M}_{\mathrm{w}}\right)$ was achieved at the rotational speed of $250 \mathrm{rpm}$ and further increase in the speed up to $350 \mathrm{rpm}$ decreased $\mathrm{M}_{\mathrm{w}}$ compared to the values at $250 \mathrm{rpm}$. Again, similar to the conversion-time profiles, the most efficient pumping action of the pitched-blade occurred at the speed of $250 \mathrm{rpm}$ with the rapid return of fluid to the impeller zone (Holland and Chapman, 1966; Spicer et al., 1996). It can be concluded that at $250 \mathrm{rpm}$ the homogeneity of the mixture was at its optimum when the pitchedblade turbine was employed. Drop in the molecular weight at the pitched-blade speed of $350 \mathrm{rpm}$ compared to $250 \mathrm{rpm}$ are due to the instability and formation of vortices and dead zones in the bulk mixture at vigorous stirring rate. As mentioned earlier in this discussion, for MMA monomer, the nucleation mechanism can be considered micellar which means the growth of polymer chain can occur inside the micelles. The emulsification and homogeneity of dispersion is very important in the final molecular weight of polymers as it raises the probability of absorption of monomer into the micelles. In Figure 5.2-3a, with employment of two pitchedblade impeller, the weight average molecular weight at low stirring rate of $100 \mathrm{rpm}$ was significantly elevated. Use of the two pitched-blade impellers increased $\mathrm{M}_{\mathrm{w}}$ at higher agitation rates of 250 and $350 \mathrm{rpm}$, but not as much as the increment seen at the low agitation rate of 100 rpm. Again, similar to the conversion profile trends, it can be resulted that bulk homogeneity did not have much change with the use of double pitched-blade instead of single one at higher impeller speeds. 
In Figure 5.2-3b, the weight average molecular weight was higher at the Rushton impeller speed of $100 \mathrm{rpm}$ compared to the similar profile using the pitched-blade turbine. The radial flow near the impeller region created a relatively good shear at low speed of $100 \mathrm{rpm}$. With increment in the Rushton impeller speed, the weight average molecular weight was enhanced at $250 \mathrm{rpm}$ and $350 \mathrm{rpm}$ due to formation of higher shear near the impeller region and the improved mixing between the two loops. However, the increase in $M_{w}$ with variation of speed was not eloquent compared to the runs with the pitched-blade impeller. Powerful pumping of the axial flow impeller, i.e. the pitched-blade was more efficient at high impeller speeds to increase the growth rate of polymeric chain. Besides, the use of double Rushton impellers as shown in Figure 5.2-3b contributed to the increase in molecular weight, but it was trivial. Therefore, producing more circulating loops did not result in a drastic increase in molecular weight. According to the data depicted in Figure 5.2-3b, when the Rushton impeller was engaged, the slope of $\mathrm{M}_{\mathrm{w}}$ versus conversion was decreased for conversions beyond $40 \%$. However, the raise in molecular weight was still perceived after the $40 \%$ conversion with a very small slope. A polymer chain grows until a second radical enters into the polymer particle to terminate with the growing one. Therefore, the chain length is inversely proportional to the radical entry frequency. For a given initiator concentration, the frequency of radical entry decreases with the number of particles, therefore the molecular weight increases (Asua, 2007). Figure 5.2-3 shows that when the pitched impeller was used, the molecular weight gradient was greater at higher impeller speeds compared to those achieved with the Rushton impeller. It can be inferred that the entry of radicals into particle was less pronounced when the pitched impeller was used compared to that measured for the Rushton impeller. 
Figure 5.2-4 shows the weight average molecular weight of the last polymer samples at the reaction time of 130 min versus impeller speed for both single and double of pitched-blade and Rushton impellers. The variation of $\mathrm{M}_{\mathrm{w}}$ with the impeller speed is more pronounced when the pitched-blade turbine is employed. The use of double impeller was more observed at low impeller speed of $100 \mathrm{rpm}$ and for the pitched-blade impeller.

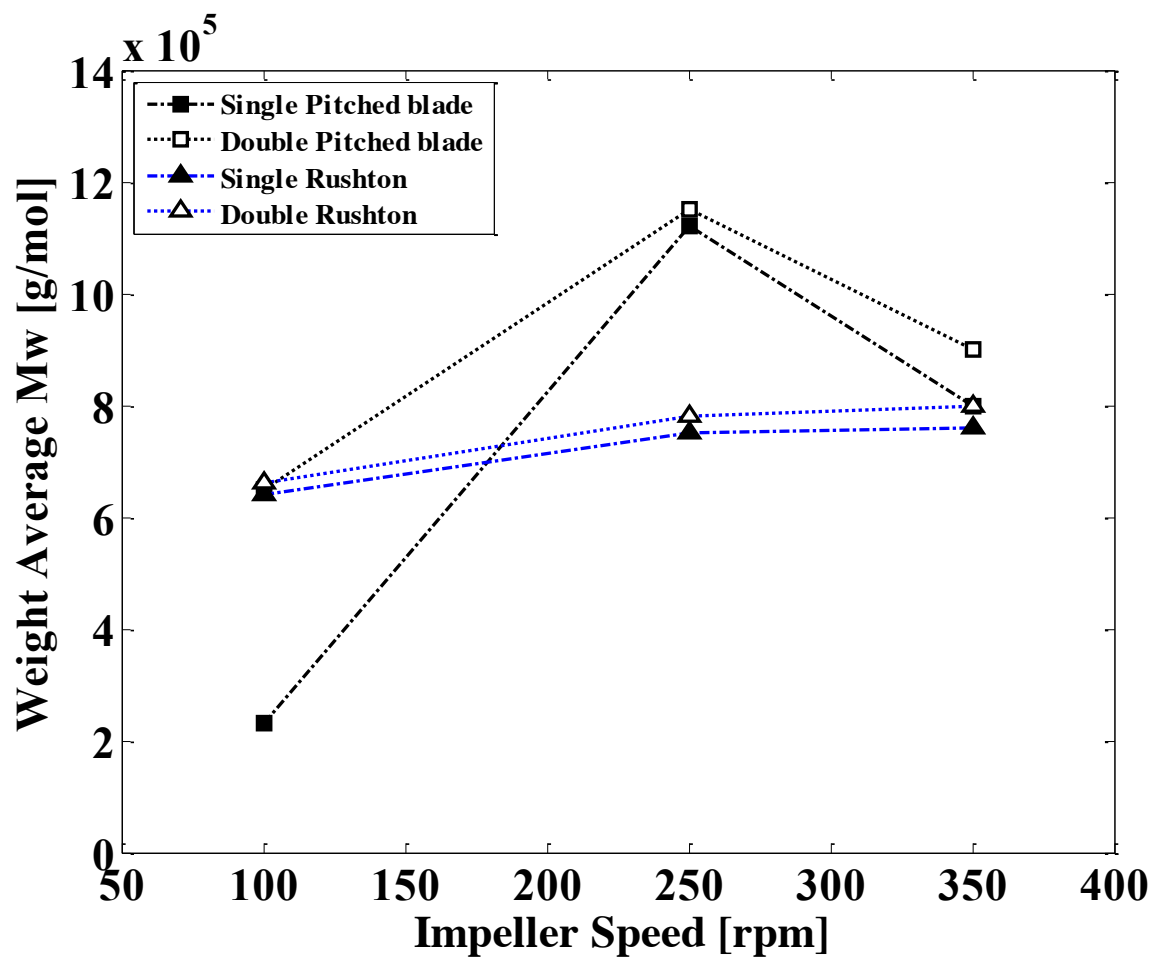

Figure 5.2-4 Final weight average molecular weight versus impeller speed at reaction time of 130 min for single/double pitched blade and Rushton impellers.

Figure 5.2-5 shows plots of polydispersity (PDI) of the last polymer samples obtained by GPC analysis at the end of polymerization (i.e. the reaction time of $130 \mathrm{~min}$ ) for both pitched-blade and Rushton impellers.

The polymers produced in the reactor equipped with the Rushton impeller had a lower PDI compared to the samples obtained using the pitched-blade turbine. This demonstrates that the narrower molecular weight distribution can be attained using the radial-flow Rushton impeller 


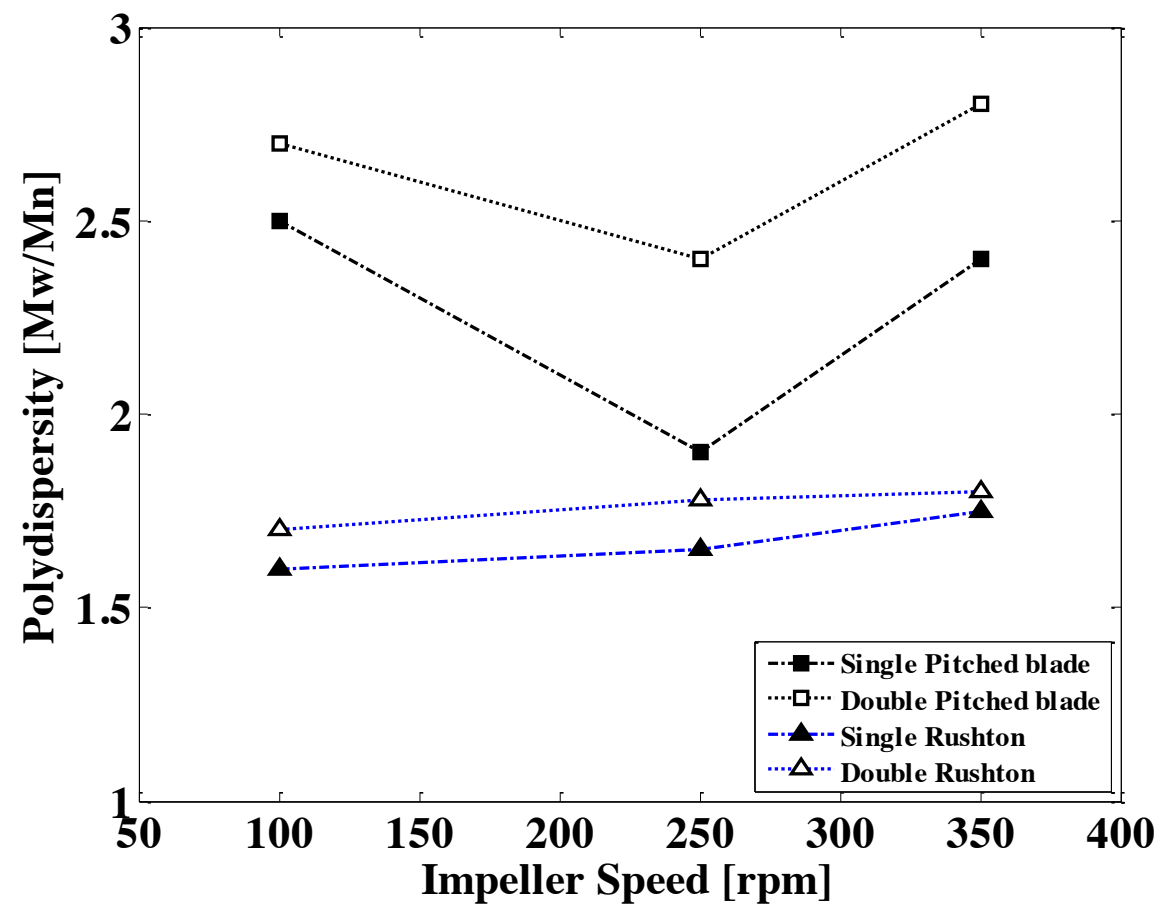

Figure 5.2-5 Final polymer poly dispersity versus impeller speed at reaction time of 130 min for single/double pitched blade and Rushton impellers.

even though the molecular weight is lower. In other words the molecular weight was better controlled by the use of the radial-flow Rushton turbine as the impeller. Besides, a uniform distribution of the molecular weight was achieved at the optimum speed of $250 \mathrm{rpm}$ for the pitched impeller. It can be implied that the existence of radicals in the particles was wellproportioned throughout the particles at this agitation rate. Therefore, it can be postulated that the growth of polymer chains and termination of polymerization were uniform in the population of particles. In other words, only the better mixing and the more uniform distribution of reaction mixture in the reactor can describe this peculiarity in the system. Yet, the GPC sampling purification method might influence this result as well. 
Figure 5.2-6 depicts the data trend of the particles size profiles versus conversion at different impeller speeds for the single and double pitched-blade (Figure 5.2-6a) and Rushton (Figure 5.26b) impellers. The samples were taken at reaction times of 5, 25, 75 and $130 \mathrm{~min}$.

In Figure 5.2-6, the trend of dependence of particle size on variation of speed was similar to the profiles of molecular weight. In Figure 5.2-6a, at the agitation rate of $100 \mathrm{rpm}$, the mean particle size was low. Again it was attributed to weak pumping of fluid and poor emulsification at this stirring rate when the pitched-blade is used. The emulsifier or surfactant molecules required to stabilize the primary particles come from those dissolved in the continuous aqueous phase and those adsorbed on the emulsified monomer droplet surfaces (Chern, 2006). In Figure 5.2-6a, when the single pitched impeller was engaged, the larger particle size was observed at the agitation rate of $250 \mathrm{rpm}$ compared to the single pitched impeller at the higher speed rate, i.e. $350 \mathrm{rpm}$.

The maximum particle size was observed at the agitation rate of $250 \mathrm{rpm}$ rather than $350 \mathrm{rpm}$. At $250 \mathrm{rpm}$ the strong pumping capacity increased bulk flow and incorporated more monomer for the top layer of the reaction mixture in the reactor vessel. In micro scale, agitation at $250 \mathrm{rpm}$ increased the growth of polymer chains by diffusion of monomer in to the micelles. At the speed of $350 \mathrm{rpm}$, as discussed before, the excessive speed produced vortices that dropped the particle sizes. Also, employment of two pitched-blade impellers increased particle sizes at higher agitation rates of 250 and $350 \mathrm{rpm}$, but the increment seen at the low agitation rate of $100 \mathrm{rpm}$ was more significant. In Figure 5.2-6b, the particle size was higher at the Rushton impeller speed of $100 \mathrm{rpm}$ compared to the same speed using the pitched-blade turbine (Figure 5.2-6a). Again, 
a)

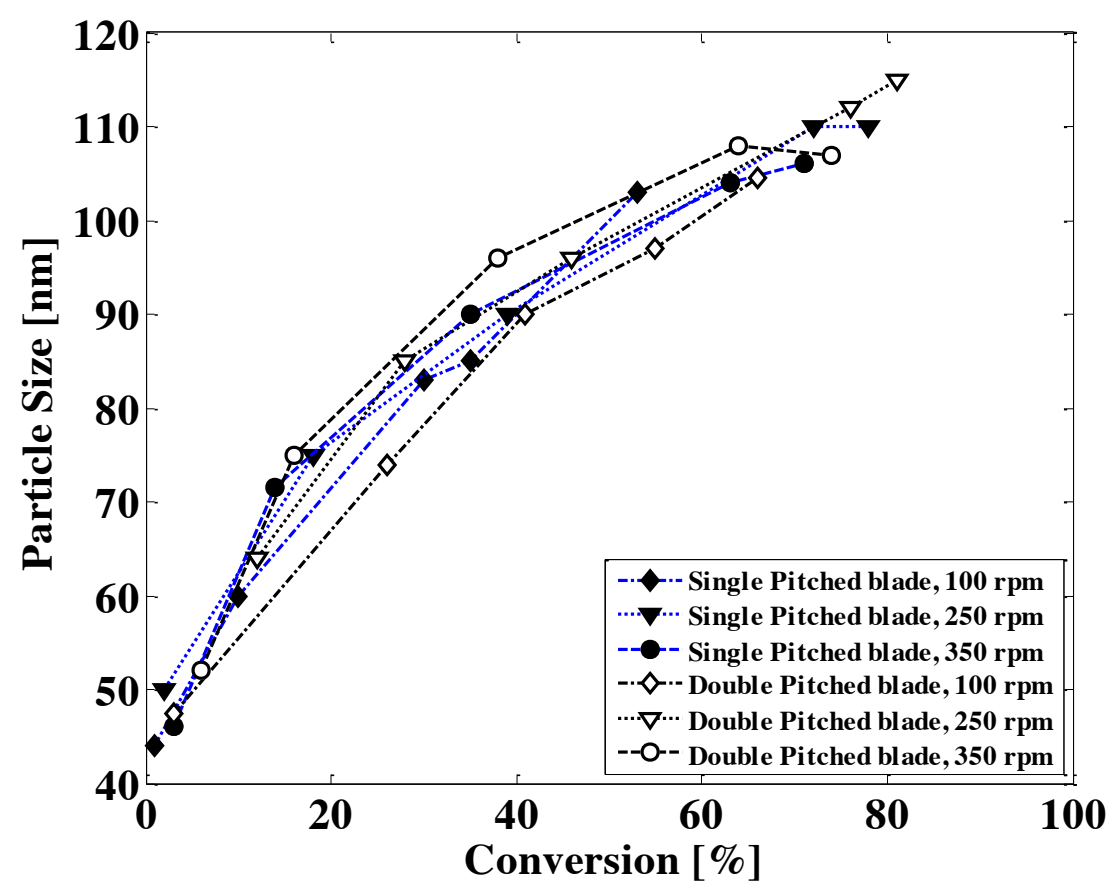

b)

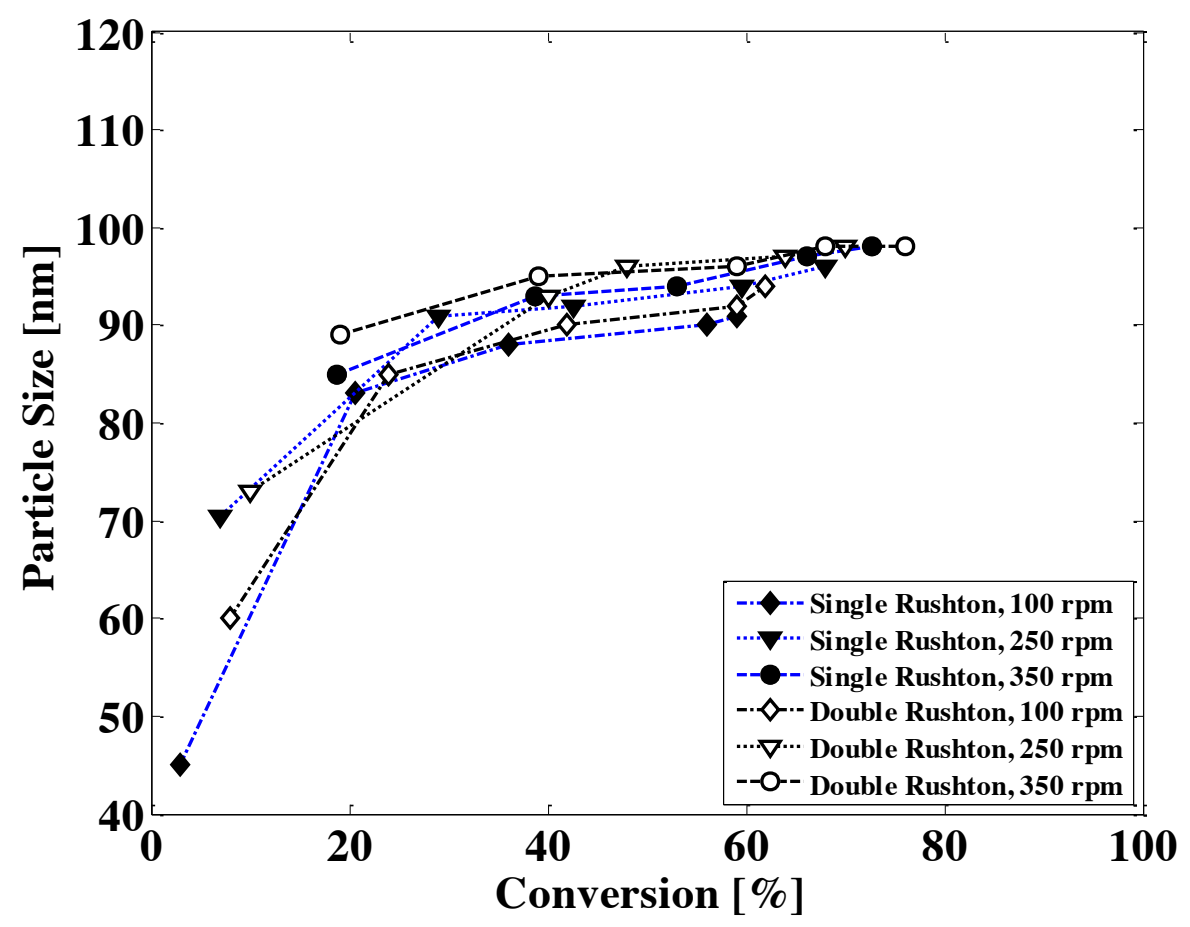

Figure 5.2-6 Mean particle size versus monomer conversion for a) pitched blade turbine and b) Rushton impeller at the speeds of 100-350 rpm using both single and double impellers. 
the relatively good mixing in the impeller region resulted in better growth of particles. However, increase in the particle size in higher speed of 250 and $350 \mathrm{rpm}$ compared with the sizes in 100 rpm was trivial. The particle size changes from the beginning to the end of the experiment were more significant for the experiments performed with the pitched impeller. When Rushton impeller was employed the slope of the particle size versus conversion drastically decreased after $20 \%$ conversion and the particles size reached a plateau after reaction times of $130 \mathrm{~min}$. In contrast, with the use of the pitched impeller, the trend of the particle size profile showed a continuous increase with a raise in conversion. Therefore, the impact of impeller configuration on the trend of particle size is more eloquent when the pitched impeller is employed.

Figure 5.2-7 shows the last mean polymer particle size versus impeller speed for both pitchedblade and Rushton impellers (single and double) at reaction time of 130 minutes. The highest particle size was seen at the pitched-blade impeller speed of $250 \mathrm{rpm}$ and moreover, using two impellers at this agitation rate resulted in a considerable increase in the mean particle sizes. Nevertheless, by comparing the final weight average molecular weight of polymers in Figure 5.2-4 with the final mean particle size of samples in Figure 5.2-7, we notice while the elevation in particle size by double pitched-blade at $250 \mathrm{rpm}$ was considerable (Figure 5.2-7), the gain in molecular weight with double pitched-blade at $250 \mathrm{rpm}$ was not very significant (Figure 5.2-4). Therefore, it is possible that agglomeration occurred at $250 \mathrm{rpm}$ when double pitched impeller was employed. However, at higher speed, i.e. $350 \mathrm{rpm}$, there is no significant difference between single and double pitched impellers.

Figure 5.2-8 shows the particle size distribution at the reaction time of 25, 75 and 130 min for the pitched-blade and Rushton turbine at the impeller speed of $250 \mathrm{rpm}$. With increase in reaction time, the particle size distribution became broader as a wide variety of polymer particles were 
produced by both types of impellers. Using the Rushton impeller resulted in lower particle size compared to the pitched-blade turbine as discussed earlier. Nevertheless, with the Rushton impeller, the particle size distribution was narrower compared to that achieved using the pitchedblade turbine.

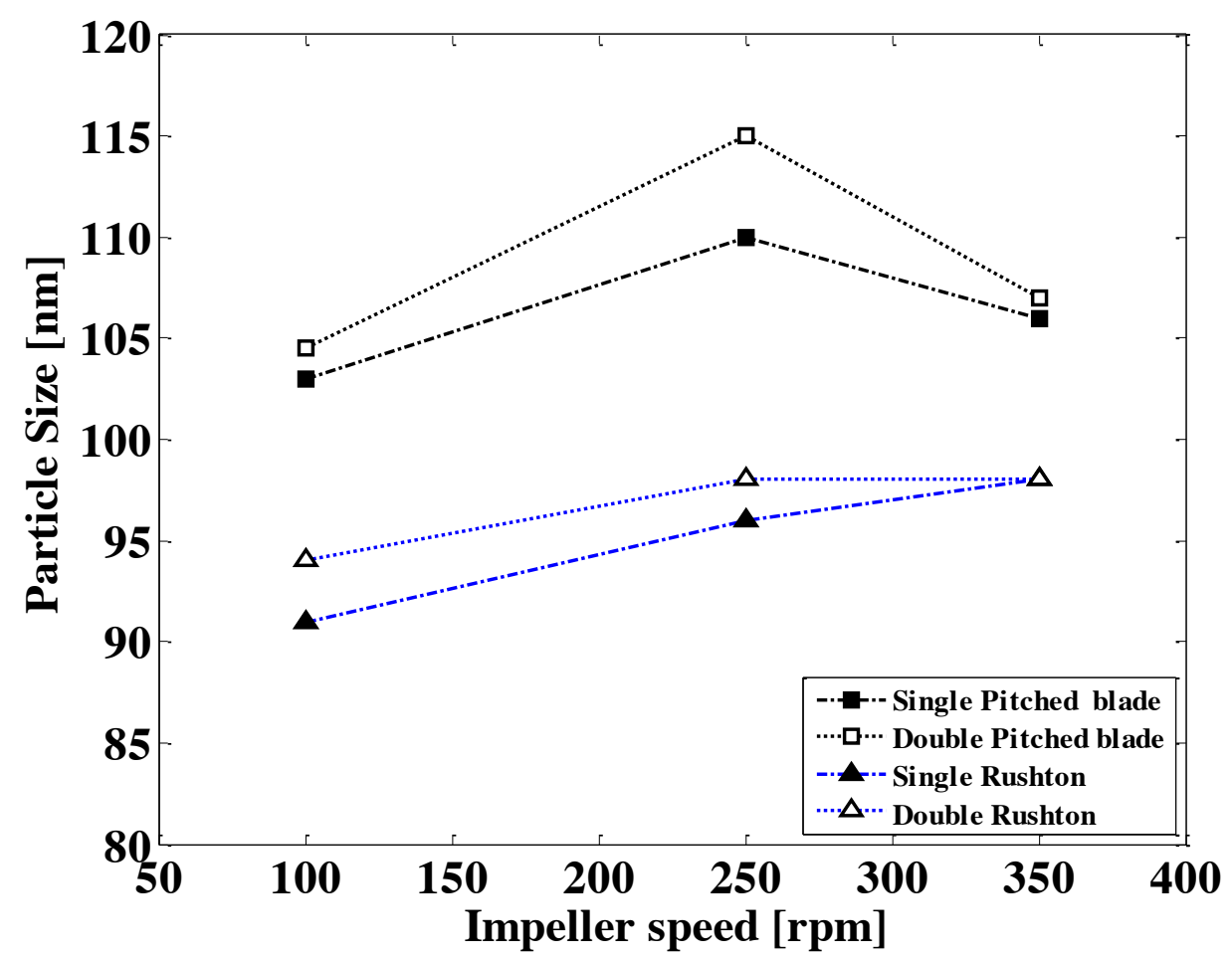

Figure 5.2-7 Final mean particle size versus impeller speed at reaction time of 130 min for single/double pitched blade and Rushton impellers.

This can be due to more intense energy dissipation in the Rushton swept volume, resulting in more stability in particle size distribution and less coalescence (Pacek et al., 1999). Moreover, although the Rushton impeller results in a lower frequency of exposure to the impeller zone in the stirred tank than the pitched-blade turbine, the higher shearing action of radial than the axial impeller in the impeller zone can result in more uniform particle size distribution (Spicer et al., 1996). According to the Microtrac Particle Analyzer manual, the standard deviation can be used 
to evaluate the width of the particle size distribution. This value for the measurement with the pitched impeller was $1.5,0.7$ and 1.0 for the impeller speed of 100, 250 and $350 \mathrm{rpm}$, which is interpreted as poorly sorted, moderately well sorted, and moderately sorted particle size

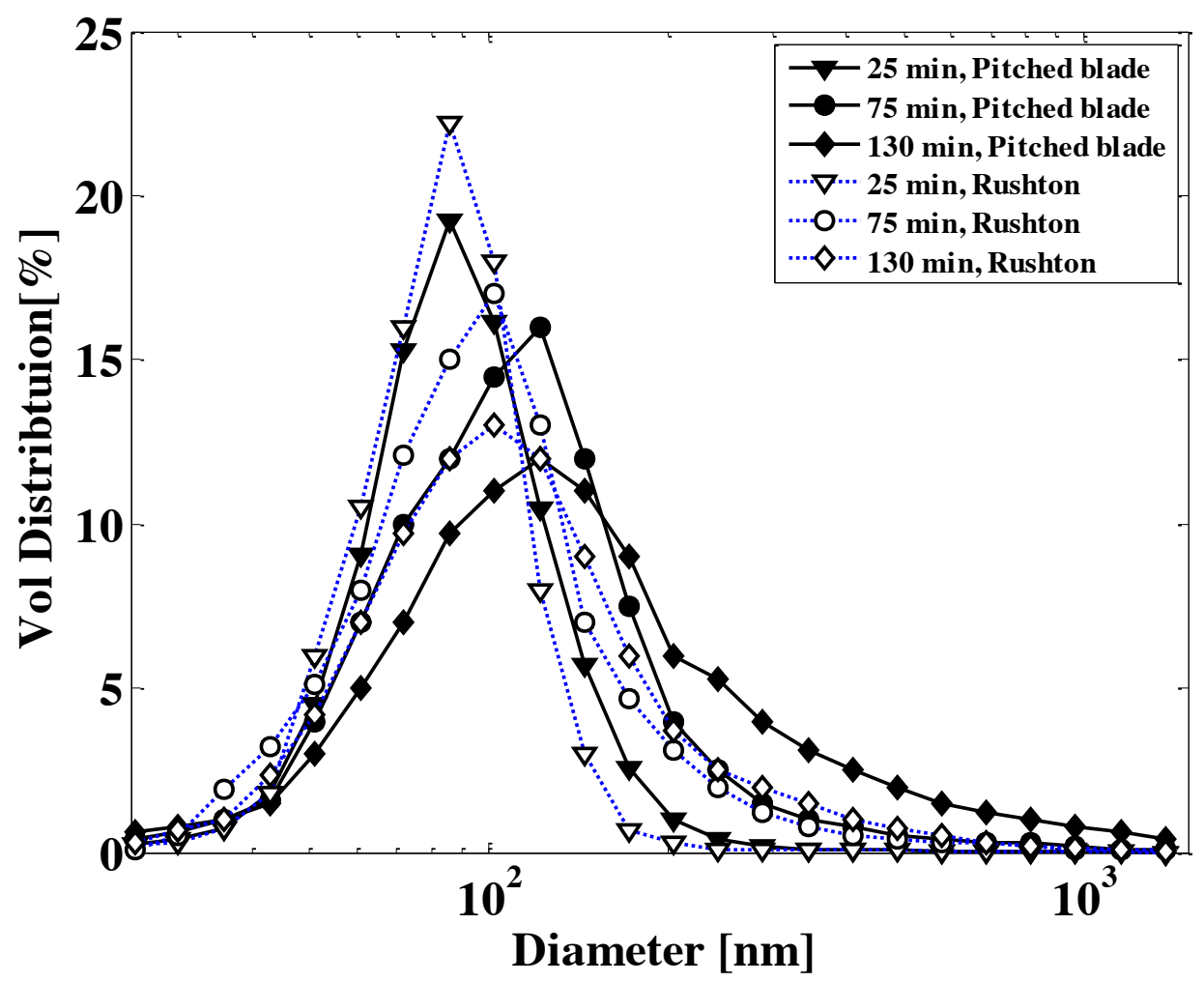

Figure 5.2-8 Particle size distribution at reaction times of 25, 75 and $130 \mathrm{~min}$ using pitched blade impeller and Rushton turbine at the impeller speed of $250 \mathrm{rpm}$.

distribution. For the measurements with the Rushton impeller, the standard deviation values were all in the range of $0.5-0.71$, which is interpreted as moderately well sorted size distribution.

The glass transition temperature $\left(T_{g}\right)$, which is an important characteristics of polymers, defines the transition region in which the polymer changes from soft to brittle, and then hard states. The $\mathrm{T}_{\mathrm{g}}$ values for the dried polymer samples were determined from the second scan of the DSC curve. Figure 5.2-9 shows the heat flow versus temperature for polymer samples obtained using 
the pitched-blade and Rushton impellers at speeds of 100-350 rpm. The variation in glass temperature $\left(T_{g}\right)$ values were recorded for the runs conducted using the pitched-blade turbine at $82.65^{\circ} \mathrm{C}, 115.44^{\circ} \mathrm{C}$ and $103.35^{\circ} \mathrm{C}$ at the impeller speeds of 100,250 and $350 \mathrm{rpm}$ respectively. The trend of gain in $T_{g}$ up to $250 \mathrm{rpm}$ and then decrease at the $350 \mathrm{rpm}$ with the use of the pitched-blade turbine is similar to the trend of molecular weight as discussed earlier. However, the glass temperatures of PMMA samples produced by employing the Rushton turbine were less affected by the impeller speed variation. The glass temperature was recorded at $89.5^{\circ} \mathrm{C}, 96.06^{\circ} \mathrm{C}$ and $101.53^{\circ} \mathrm{C}$ at the impeller speeds of 100,250 and $350 \mathrm{rpm}$ respectively. The dependence of glass temperature with the average molecular has been reported in literature as well (Blanchard et al., 1974).

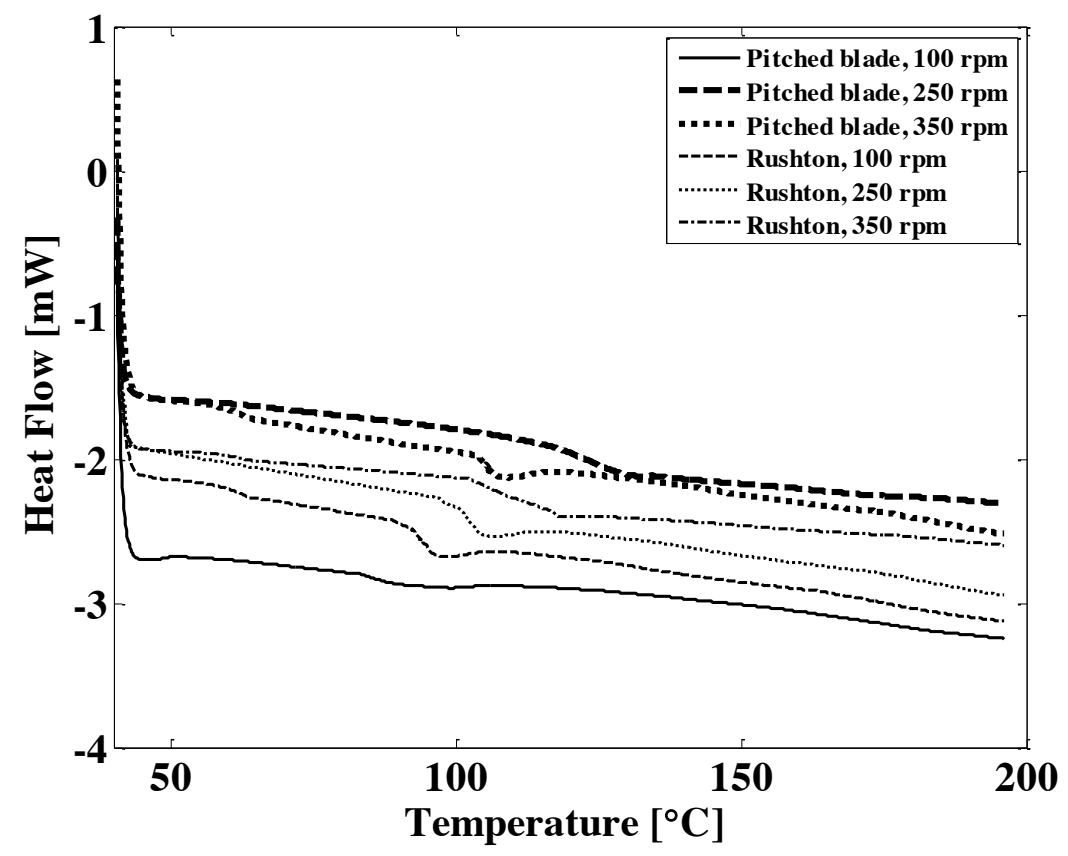

Figure 5.2-9 Heat flow versus temperature for final polymer at the impeller speeds of 100-350 rpm using pitched blade turbine and Rushton impeller. 
A general linear model was performed using Minitab-16 to generate the analysis of variance (ANOVA) Tables for conversion, molecular weight and particle size, respectively. The p-value was used to judge whether F-statistics was large enough to indicate statistical significance. A pvalue lower than 0.05 indicates that the model is considered to be statistically significant (Tshukudu et al., 2013). Table 5.2-1 demonstrates that the impeller speed was a statistically significant factor for the conversion response while the impeller type, number of impellers, and interaction factors were insignificant.

For the molecular weight response, according to Table 5.2-2, all factors and their interactions were insignificant. However, the re-analysis of the design revealed the significance of the impeller speed on the molecular weight response. Finally, according to Table 5.2-3, the impeller type and speed were statistically significant on the particle size response while their interaction factor was insignificant. 
Table 5.2-1 ANOVA results for the final conversion response.

\begin{tabular}{|c|c|c|c|c|c|c|}
\hline Source & $\begin{array}{c}\text { Degrees } \\
\text { of } \\
\text { freedom }\end{array}$ & $\begin{array}{l}\text { Sequential } \\
\text { sum of } \\
\text { squares }\end{array}$ & $\begin{array}{l}\text { Adjusted } \\
\text { sum of } \\
\text { squares }\end{array}$ & $\begin{array}{l}\text { Adjusted } \\
\text { mean } \\
\text { square }\end{array}$ & F -statistic & P-value \\
\hline Impeller speed & 2 & 511.629 & 511.629 & 255.815 & 32.36 & 0.030 \\
\hline Impeller type & 1 & 19.610 & 19.610 & 19.610 & 2.48 & 0.256 \\
\hline Number of impellers & 1 & 62.290 & 62.290 & 62.290 & 7.88 & 0.107 \\
\hline Speed * Impeller type & 2 & 94.989 & 94.989 & 47.495 & 6.01 & 0.143 \\
\hline Speed $*$ Number of impellers & 2 & 18.009 & 18.009 & 9.005 & 1.14 & 0.467 \\
\hline Impeller type * Number of impellers & 1 & 9.470 & 9.470 & 9.470 & 1.20 & 0.388 \\
\hline Error & 2 & 15.809 & 15.809 & 7.905 & & \\
\hline Total & 11 & 731.806 & & & & \\
\hline \multicolumn{7}{|c|}{ Standard deviation $=2.81152 \quad$ R-Square $=97.84 \% \quad$ R-Square $($ adjusted $)=88.12 \%$} \\
\hline \multicolumn{7}{|c|}{$\begin{array}{l}\text { Regression model: } \\
\text { Conversion }=69.2217-9.222 * \text { (Impeller speed)- } 1.2783 *(\text { Impeller type })-2.2783 * \text { (Number of impellers) }-3.972 * \\
\text { (Impeller type) } * \text { (Impeller speed) }-1.722 * \text { (Impeller speed }) *(\text { Number of impellers })+0.8883 * \text { (Impeller type }) *(\text { Number } \\
\text { of impellers) }\end{array}$} \\
\hline \multicolumn{7}{|c|}{ After Re-analysis } \\
\hline Source & $\begin{array}{c}\text { Degrees } \\
\text { of } \\
\text { freedom }\end{array}$ & $\begin{array}{l}\text { Sequential } \\
\text { sum of } \\
\text { squares }\end{array}$ & $\begin{array}{c}\text { Adjusted } \\
\text { sum of } \\
\text { squares }\end{array}$ & $\begin{array}{l}\text { Adjusted } \\
\text { mean } \\
\text { square }\end{array}$ & F -statistic & P-value \\
\hline Impeller speed & 2 & 511.63 & 511.63 & 255.81 & 10.46 & 0.004 \\
\hline Error & 9 & 220.18 & 220.18 & 24.46 & & \\
\hline Total & 11 & 731.81 & & & & \\
\hline
\end{tabular}


Table 5.2-2 ANOVA results for the final molecular weight response.

\begin{tabular}{|c|c|c|c|c|c|c|}
\hline Source & $\begin{array}{c}\begin{array}{c}\text { Degrees } \\
\text { of } \\
\text { freedom }\end{array} \\
\end{array}$ & $\begin{array}{c}\text { Sequential } \\
\text { sum of } \\
\text { squares } \\
\end{array}$ & $\begin{array}{c}\text { Adjusted } \\
\text { sum of } \\
\text { squares } \\
\end{array}$ & $\begin{array}{c}\text { Adjusted } \\
\text { mean } \\
\text { square } \\
\end{array}$ & F -statistic & P-value \\
\hline Impeller speed & 2 & $3.4065 \mathrm{e}+11$ & $3.4065 \mathrm{e}+11$ & $1.7033 \mathrm{e}+11$ & 14.72 & 0.064 \\
\hline Impeller type & 1 & $1.771 \mathrm{e}+10$ & $1.771 \mathrm{e}+10$ & $1.771 \mathrm{e}+10$ & 1.53 & 0.342 \\
\hline Number of impellers & 1 & $3.424 \mathrm{e}+11$ & $3.424 \mathrm{e}+11$ & $3.424 \mathrm{e}+11$ & 2.96 & 0.228 \\
\hline Speed * Impeller type & 2 & $1.6798 \mathrm{e}+11$ & $1.6798 \mathrm{e}+11$ & $8.399 \mathrm{e}+10$ & 7.26 & 0.121 \\
\hline Speed $*$ Number of impellers & 2 & $2.018 \mathrm{e}+10$ & $2.018 \mathrm{e}+10$ & $1.009 \mathrm{e}+10$ & 0.87 & 0.534 \\
\hline Impeller type * Number of impellers & 1 & $1.756 \mathrm{e}+10$ & $1.756 \mathrm{e}+10$ & $1.756 \mathrm{e}+10$ & 1.52 & 0.343 \\
\hline Error & 2 & $2.314 \mathrm{e}+10$ & $2.314 \mathrm{e}+10$ & 115.7 & & \\
\hline Total & 11 & $6.2146 \mathrm{e}+11$ & & & & \\
\hline \multicolumn{7}{|c|}{ Standard deviation $=107572$ R-Square $=96.28 \% \quad$ R-Square $($ adjusted $)=79.52 \%$} \\
\hline \multicolumn{7}{|c|}{$\begin{array}{l}\text { Regression model: } \\
\text { Molecular weight }=769917-225167 *(\text { Impeller speed })+38417 *(\text { Impeller type })+53417 *(\text { Number of impellers })+146583 * \\
(\text { Impeller type } *(\text { Impeller speed })+56833 * \text { (Impeller speed }) *(\text { Number of impellers })+38250 *(\text { Impeller type }) *(\text { Number of } \\
\text { impellers })\end{array}$} \\
\hline \multicolumn{7}{|c|}{ After Re-analysis } \\
\hline Source & $\begin{array}{c}\text { Degrees } \\
\text { of } \\
\text { freedom }\end{array}$ & $\begin{array}{c}\text { Sequential } \\
\text { sum of } \\
\text { squares } \\
\end{array}$ & $\begin{array}{c}\text { Adjusted } \\
\text { sum of } \\
\text { squares } \\
\end{array}$ & $\begin{array}{c}\text { Adjusted } \\
\text { mean } \\
\text { square } \\
\end{array}$ & F -statistic & P-value \\
\hline Impeller speed & 2 & $3.40650 \mathrm{e}+11$ & $3.40650 \mathrm{e}+11$ & $1.70325 \mathrm{e}+11$ & 5.46 & 0.028 \\
\hline Error & 9 & $2.80811 \mathrm{e}+11$ & $2.80811 \mathrm{e}+11$ & $3.1201 \mathrm{e}+11$ & & \\
\hline Total & 11 & $6.21461 \mathrm{e}+11$ & & & & \\
\hline \multicolumn{2}{|c|}{ Standard deviation $=176639 \quad$ R-Square $=54.81 \%$} & \multicolumn{5}{|c|}{ R-Square $($ adjusted $)=44.77 \%$} \\
\hline
\end{tabular}


Table 5.2-3 ANOVA results for the final particle size response.

\begin{tabular}{|c|c|c|c|c|c|c|}
\hline Source & $\begin{array}{l}\text { Degrees } \\
\text { of } \\
\text { freedom }\end{array}$ & $\begin{array}{l}\text { Sequential } \\
\text { sum of } \\
\text { squares }\end{array}$ & $\begin{array}{l}\text { Adjusted } \\
\text { sum of } \\
\text { squares }\end{array}$ & $\begin{array}{l}\text { Adjusted } \\
\text { mean } \\
\text { square }\end{array}$ & F -statistic & P-value \\
\hline Impeller speed & 2 & 89.542 & 89.542 & 44.771 & 35.23 & 0.028 \\
\hline Impeller type & 1 & 414.188 & 414.188 & 414.188 & 325.92 & 0.003 \\
\hline Number of impellers & 1 & 13.021 & 13.021 & 13.021 & 10.25 & 0.085 \\
\hline Speed * Impeller type & 2 & 24.875 & 24.875 & 12.438 & 9.79 & 0.093 \\
\hline Speed $*$ Number of impellers & 2 & 4.542 & 4.542 & 2.271 & 1.79 & 0.359 \\
\hline Impeller Type * Number of impellers & 1 & 0.521 & 0.521 & 0.521 & 0.41 & 0.588 \\
\hline Error & 2 & 2.542 & 2.542 & 1.271 & & \\
\hline Total & 11 & 549.229 & & & & \\
\hline Standard deviation $=1.12731 \quad$ R-Squ & $\mathrm{re}=99.54 \%$ & R-Square $(\mathrm{ad}$ & sted) $=97.45$ & & & \\
\hline \multicolumn{7}{|c|}{$\begin{array}{l}\text { Regression model: } \\
\text { Particle size }=101.708-3.5833 *(\text { Impeller speed })+5.8750 *(\text { Impeller type })+1.0417 *(\text { Number of impellers })-0.2500 * \\
(\text { Impeller type }) *(\text { Impeller speed })-1.722 *(\text { Impeller speed }) *(\text { Number of impellers }) \\
+0.8883 *(\text { Impeller type }) *(\text { Number of impellers })\end{array}$} \\
\hline \multicolumn{7}{|c|}{ After Re-analysis } \\
\hline Source & $\begin{array}{l}\text { Degrees } \\
\text { of } \\
\text { freedom }\end{array}$ & $\begin{array}{l}\text { Sequential } \\
\text { sum of } \\
\text { squares }\end{array}$ & $\begin{array}{l}\text { Adjusted } \\
\text { sum of } \\
\text { squares }\end{array}$ & $\begin{array}{l}\text { Adjusted } \\
\text { mean } \\
\text { square }\end{array}$ & F -statistic & P-value \\
\hline Impeller type & 1 & 414.19 & 414.19 & 414.19 & 120.49 & 0.000 \\
\hline Impeller speed & 2 & 89.54 & 89.54 & 44.77 & 13.02 & 0.007 \\
\hline Impeller Type*Impeller speed & 2 & 24.88 & 24.88 & 12.44 & 3.62 & 0.093 \\
\hline Error & 6 & 20.63 & 20.63 & 3.44 & & \\
\hline Total & 11 & 549.23 & & & & \\
\hline \multicolumn{7}{|c|}{ Standard deviation $=1.85405$ R-Square $=96.24 \% \quad$ R-Square $($ adjusted $)=93.12 \%$} \\
\hline \multicolumn{7}{|c|}{$\begin{array}{l}\text { Regression model: } \\
\text { Particle size }=101.708+5.8750 *(\text { Impeller type })-3.5833 *(\text { Impeller speed })+1.8750 *(\text { Impeller type }) *(\text { Impeller speed })\end{array}$} \\
\hline
\end{tabular}


Finally, Figure 5.2-10 shows the scanning electron microscopy (SEM) image (Model: Hitachi SU8000) of the dried end product latex which was obtained at the Rushton turbine speed of 250 rpm.

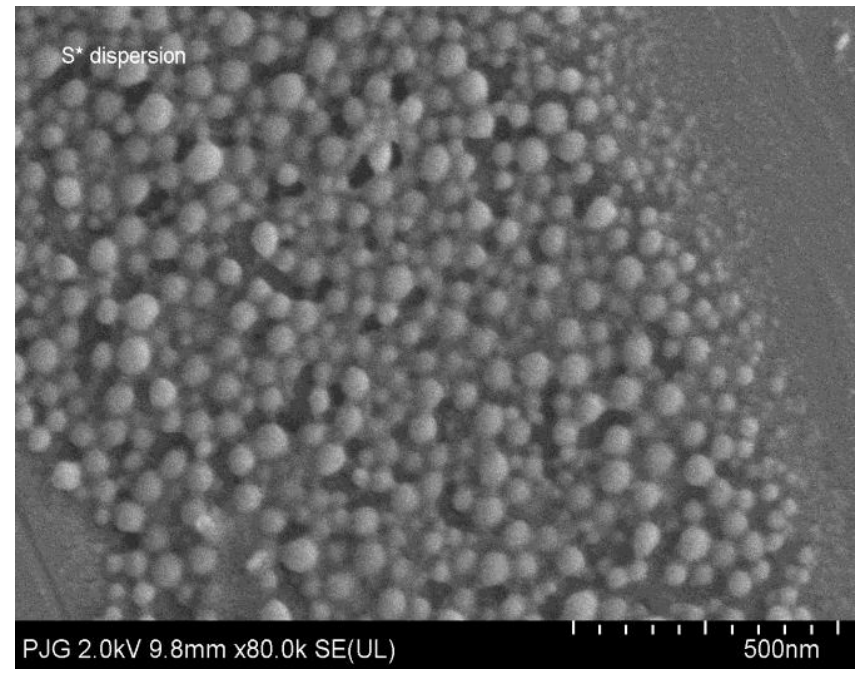

Figure 5.2-10 SEM image of final polymer product using Rushton turbine at impeller speed 250 rpm.

This image shows the size and shape distributions of the dried polymer particles. The information obtained in this study has combined the mixing phenomena with polymer chemistry to progress the emulsion polymerization process to a point where industrial applications such as scale up and reactor design are much more feasible. The computational fluid dynamics (CFD) modeling in the research lab were utilized to study the details of mixing such as shear forces, turbulence, and flow pattern within the reaction mixture. 


\subsubsection{Concluding remarks}

Emulsion polymerization of MMA was carried out in a 2L lab reactor equipment with two different impeller types, a six pitched-blade and Rushton impeller. The effects of number of impellers, speed, and type of impellers on MMA conversion, molecular weight, particle size and glass temperature were investigated. Stirring rate was varied from 100 to $350 \mathrm{rpm}$ and single or double impeller were mounted on the shaft. Using the pitched-blade turbine reduced the monomer conversion, $\mathrm{M}_{\mathrm{w}}$, mean particle size and glass temperature at low agitation rate of 100 rpm. The maximum monomer conversion, $\mathrm{M}_{\mathrm{w}}$, mean particle size and glass temperature values were obtained at $250 \mathrm{rpm}$ and further increase in speed, declined the mentioned properties.

Using the Rushton impeller showed relatively higher values for conversion, $\mathrm{M}_{\mathrm{w}}$, particle size and glass temperature compared to the values obtained using the pitched-blade turbine at 100 rpm; however, the increase in the mentioned properties with the elevation of impeller speed was less significant than the gain obtained using the pitched-blade turbine.

With employment of two pitched-blade impeller, the monomer conversion, $\mathrm{M}_{\mathrm{w}}$ and particle size at low stirring rate of $100 \mathrm{rpm}$ were elevated compared to values obtained using single pitchedblade impeller. However, at higher agitation rates, the increments were insignificant. Employment of double Rushton impellers was not so effective in increasing the monomer conversion, $\mathrm{M}_{\mathrm{w}}$ and particle size. Using Rushton impeller resulted in the narrower molecular weight and particle size distribution compared to the pitched-blade turbine.

The finding of the current study has the potential to contribute to the development of the novel polymerization processes where the adjustment of polymer characteristics can be obtained by suitable configuration design. 


\subsection{Analysis of mixing in emulsion polymerization of MMA}

\subsubsection{Introduction}

The thorough literature review revealed that little effort has been devoted to the estimation of the particle size distribution by incorporating the nucleation and growth phenomena for emulsion polymerization conducted in a stirred tank reactor through the coupling scheme between CFD and the population balance. According to comprehensive literature review, no attempt has been made to include the chemical reaction kinetics to the population balance through the nucleation and growth rates while taking into account the velocity gradients generated by the impeller rotation inside the emulsion polymerization reactor. Thus, the core objective of this study is to couple the turbulent flow generated by the impeller in a lab-scale MMA emulsion polymerization with the transport species equations, population balance with the nucleation and growth rates, and reaction kinetics. One of the important computational issues is the solution method of the transport equations coupled with the turbulence, reaction kinetics, and population balance equations, which can be sequential or simultaneous solution. In sequential technique, first the velocity flow field and the turbulence model parameters are calculated and then population balance equations are solved. On the other hand, in simultaneous solution approach, all the equations are solved at the same time. The accuracy of the obtained results is definitely influenced by the solution method. In this study, the simultaneous solution approach is employed to enhance the accuracy of the simulation results. Another significant issue is using the parallel computation. In order to calculate the particle size distribution, rather than obtaining only the mean diameter, the parallel computation is utilized in this study to reduce the extensive computational time and cost. The experimental data as described in Chapter 5, Sections 5.1 and 5.2 are also utilized for the model validation. The validated model is then employed to explore 
the effects of baffles, impeller speed and type on the particle size distribution (number density), conversion, and polymer particle size.

\subsubsection{Results and discussion}

The CFD model developed in this study was employed to explore the effects of the stirring rate, baffles, and impeller type on the monomer conversion, polymer phase volume fraction, the mean particle size, and the number density of particles. Since the population balance equations for all bins (classes) were solved simultaneously with transport equations for two phases along with the turbulence equations for $k_{t u r b}$ and $\varepsilon_{t u r b}$ and volume fraction continuity of the second phase, the number of bins (classes) was limited to seven and satisfactory results were obtained. According to the literature, sufficient number of classes should be used to minimize the discretization errors Fathi Roudsari et al., 2012; Laakkonen et al., 2007). To validate the CFD model, the computed monomer conversion, the mean particle size, and the number density for different impeller speeds and impeller types in the reactors with and without baffles were compared to the experimental data (Fathi Roudsari et al., 2014a and 2014b).The first part presents the effect of the impeller speed and baffles on conversion, number density, and particle size when the reactor was equipped with a pitched blade impeller. In the second part of the discussion, the impact of the impeller type on the mentioned latex properties is debated. It should be mentioned that the complete conversion was not achieved in the experiments. It has often been noted (Soh and Sundberg, 1982) that polymerizations carried out at temperatures significantly below the glass transition temperature of the pure polymer do not appear to reach full conversion. Besides, the limiting conversions can be attributed to the reduction in the initiator efficiency and a decrease in the decomposition rate of the initiator at the isothermal condition (Faldi and Tirrell, 1994). 
Furthermore, in this study, the monomer was not purified as it was intended to resemble the actual industrial case.

Effect of the impeller speed and baffles:

Fig. 5.3-1 shows profiles of monomer conversion as a function of the impeller speed at reaction time of $130 \mathrm{~min}$ for the pitched blade impeller at $60^{\circ} \mathrm{C}$ and for the reactors with baffles and without baffles. Experimental data are presented by white symbols and the CFD predictions by black symbols. The trends of the CFD simulations are shown by dotted lines. The un-reacted mass fraction of monomer specie was monitored during each simulation until it reached to a constant value. The monomer conversion was then calculated by dividing the mass of reacted monomer to the mass of initial monomer in the reactor.

Generally, at higher impeller speeds, the mixing of the reaction mixture is improved and the probability of the reaction between reactants and thus the rate of polymerization is enhanced (Fathi Roudsari et al., 2013, 2014a and 2014b). Besides, the initiator performance improves as the result of the improved recirculation achieved at the higher impeller speed within the reactor. As can be seen in Figure 5.3-1, the conversion was elevated by increasing the rotational speed of the pitched blade impeller from 20 to $250 \mathrm{rpm}$, however, in both reactors with and without baffles, the conversion was decreased at the impeller speed greater than $250 \mathrm{rpm}$. This trend was the same for both CFD and experimental work. The vigorous stirring at $350 \mathrm{rpm}$ induced instability in emulsion on the emulsion mass, which led to a decrease in conversion when the rotational speed was increased from 250 to $350 \mathrm{rpm}$.

Besides, as depicted in Figure 5.3-1, the use of baffles decreased the conversion by about $7 \%$ at $100 \mathrm{rpm}, 9 \%$ at $250 \mathrm{rpm}$, and by about $2-3 \%$ at $20 \mathrm{rpm}$ and $350 \mathrm{rpm}$. According to the CFD results, the use of baffles decreased the conversion by about $7 \%$ at $250 \mathrm{rpm}, 10 \%$ at $100 \mathrm{rpm}, 5 \%$ 


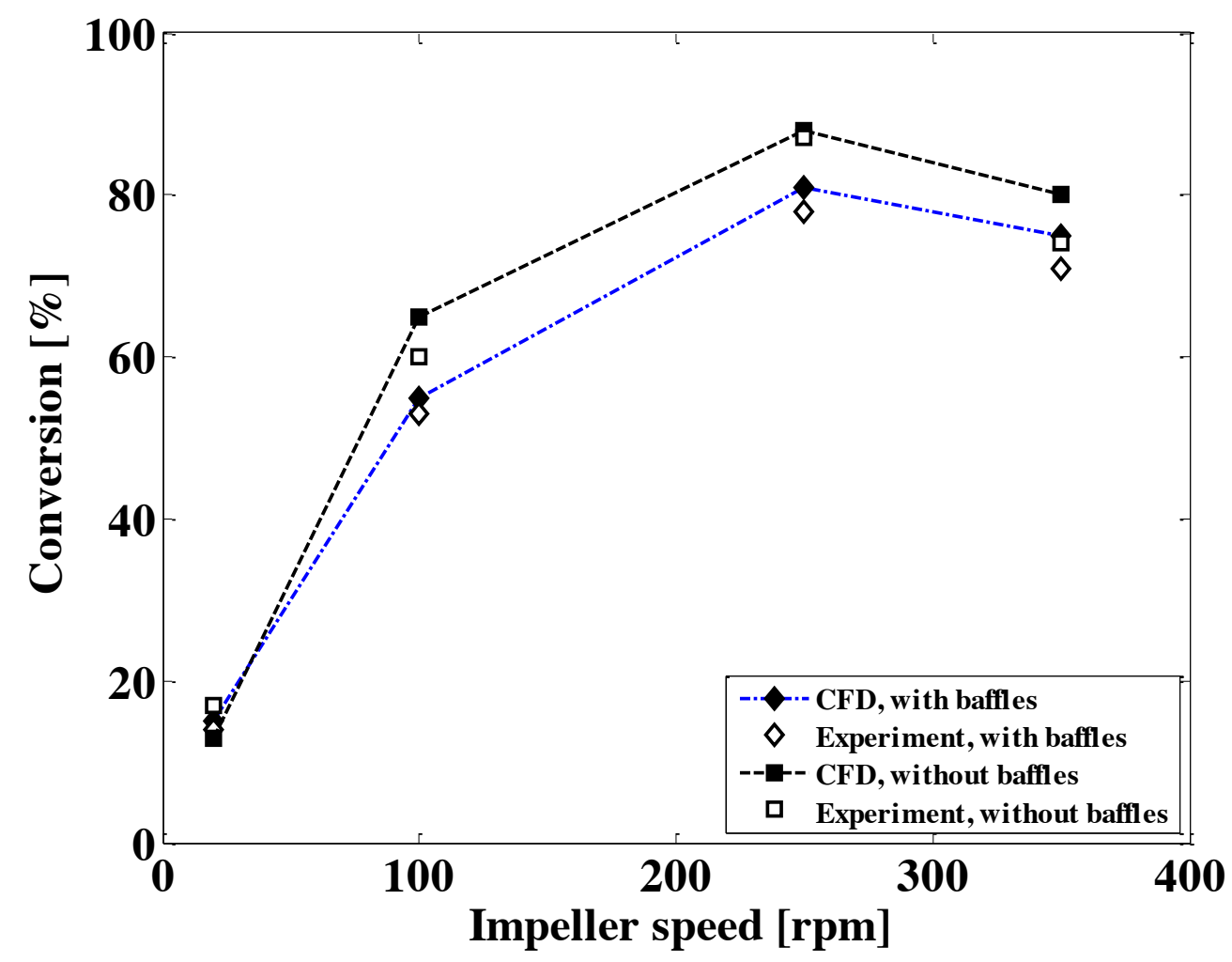

Figure 5.3-1 Monomer conversion versus impeller speed at the reaction time of 130 min for the pitched blade impeller and the reactors with baffles and without baffles.

at $350 \mathrm{rpm}$, and $2 \%$ at $20 \mathrm{rpm}$. The maximum reduction in conversion was $9 \%$ in the experiment and $10 \%$ in the CFD modeling. The decrease in conversion when the baffles were installed in the reactor can be due to the accumulation of monomer droplets behind the baffle surface since the baffles were attached to the reactor tank wall due to the restriction of available space inside the reactor. Figure 5.3-2 depicts the primary phase mixture volume fraction which is comprised of the mixture of monomer and water on the top surface in the reactor with and without baffles. It can be seen that the volume fraction of the primary phase behind the three of baffles (Figure 5.32a) was relatively higher than other regions. Besides, behind the fourth baffle, the volume fraction of monomer was very low which indicates a higher volume fraction of polymer behind 
this baffle. In contrast, in the reactor without baffles (Figure 5.3-2b), there is no accumulation of any of the two phases near the tank wall. This un-reacted monomer can be a reason for a lower conversion when the baffles were installed.

a)

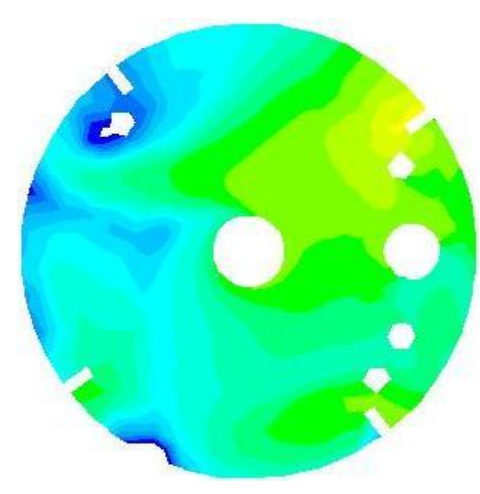

b)

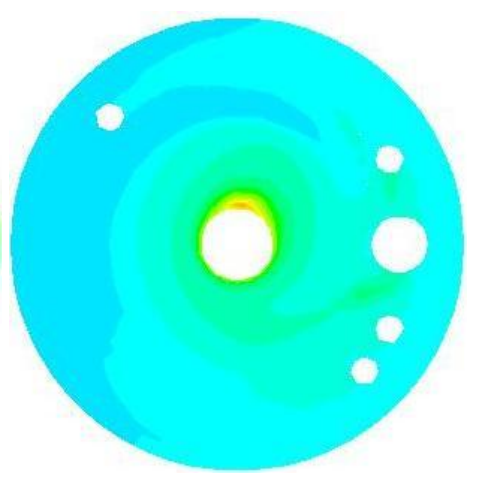

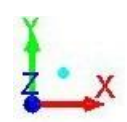
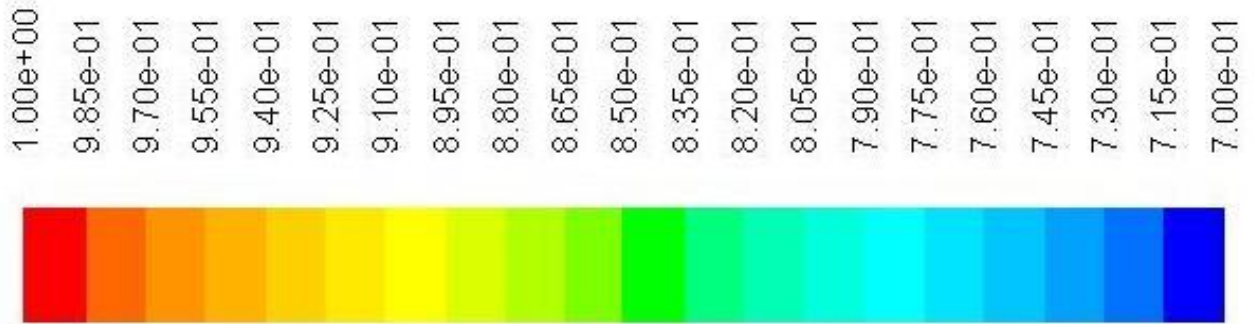

Figure 5.3-2 Contours of the first phase volume fraction on the top surface in the reactor equipped with the pitched blade impeller rotating at $250 \mathrm{rpm}$ : a) with baffles and b) without baffles.

In Figure 5.3-3 and Figure 5.3-4, the number density of particles computed through the CFD model at seven bin sizes are depicted for the reactors equipped with a pitched blade impeller with and without baffles, respectively. At the low impeller speed of $20 \mathrm{rpm}$, the number densities in all bin sizes were low. With an increase in the impeller speed to 100, 250, and $350 \mathrm{rpm}$, the number densities were enhanced. The highest number density was obtained at the bin size of 112 $\mathrm{nm}$ (bin 4) at the impeller speed of $350 \mathrm{rpm}$. Furthermore, the number densities calculated using 
the CFD model for the reactor without the baffles (Figure 5.3-4) were higher than those obtained for the reactor with baffles (Figure 5.3-3) as a result of a higher monomer conversion. As previously discussed, the conversion was lower in the reactor with baffles due to the collection of monomer behind the baffles. Figure 5.3-5 shows the mean particle size as a function of the impeller speed at reaction time of $130 \mathrm{~min}$ for the pitched blade impeller at $60^{\circ} \mathrm{C}$ and for the reactors with baffles and without baffles. Experimental data are presented by white symbols and the CFD predictions by black symbols. The trends of the CFD simulations are shown using the dotted lines. The moments obtained by population balance modeling can be calculated after the convergence was achieved in simulations.

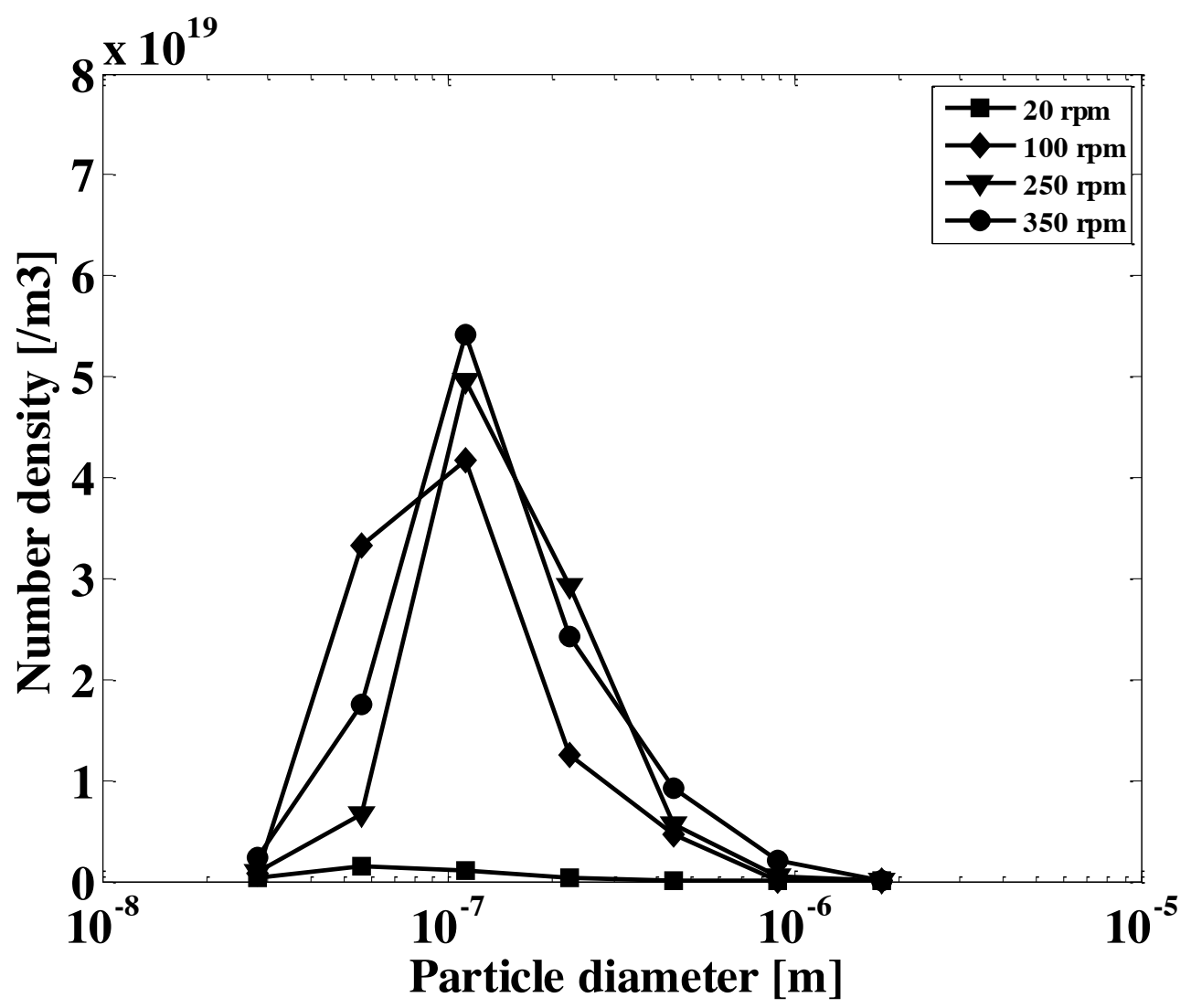

Figure 5.3-3 Number density distribution of particles computed through the CFD model for the pitched blade impeller in the reactor with the baffles at the rotational speeds of 20, 100, 250, and $350 \mathrm{rpm}$. 


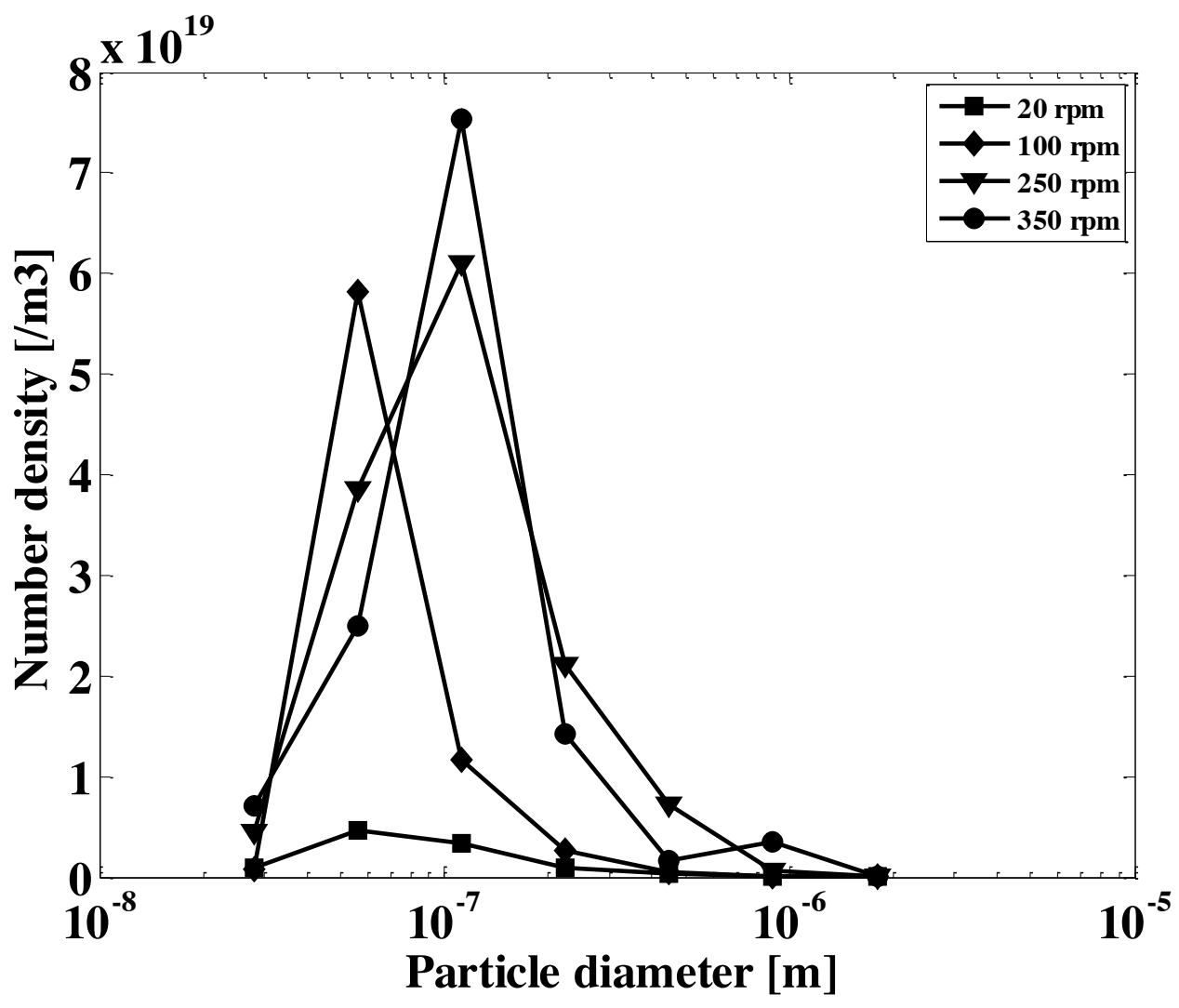

Figure 5.3-4 Number density distribution of particles computed through the CFD model for the pitched blade impeller in the reactor without baffles at the rotational speeds of 20, 100, 250, and $350 \mathrm{rpm}$.

In the CFD modeling, the mean Sauter particle size was calculated by dividing the third moment to the second moment calculated through the CFD model for each simulation. The equation used for the calculation of the mean Sauter particle size $D_{32}$ is as follows (Ansys Inc, 2010):

$$
D_{32}=\frac{\sum n_{k}^{\prime} D_{k}^{3}}{\sum n_{k}^{\prime} D_{k}^{2}}
$$

$D_{k}$ is the polymer particle diameter size in bin (class) $k$ and $n_{k}^{\prime}$ is the number of particles (number density) in bin (class) $k$. 
In emulsion polymerization, monomer can be transported to the growing latex particles by molecular diffusion from the continuous aqueous phase or by the shear induced collision between the monomer droplets and particles (Chern, 2006). Therefore, the particle size enlargement observed as a result of an improvement in mixing quality achieved with an increase in the impeller speed from $20 \mathrm{rpm}$ to $250 \mathrm{rpm}$ can be attributed to both molecular diffusion and shear induced collision mechanisms. On the other hand, a further increase in the impeller speed (e.g. greater than $250 \mathrm{rpm}$ ) resulted in a slight reduction in the particle diameters. As the surfactant concentration was constant in all runs at different mixing rates, the instability of emulsion at excessive stirring can only be due to the collision of radicals and termination reaction inside the particles, which resulted in ending the polymerization.

Generally, using the baffles increases the axial velocity component that promotes the fluid circulation and reduces the tangential or swirl velocity (Paul et al., 2004). Figure 5.3-6 depicts the contours of axial velocity at the impeller speed of $350 \mathrm{rpm}$ in the reactors with baffles and without baffles. By comparing the contours of axial velocities in Figure 5.3-6a and Figure 5.3$6 \mathrm{~b}$, the difference in the flow patterns inside the reactor with and without baffles is clearly observed which in turn influences the shear rates. Table 5.3-1 shows the volume average shear rates for different reactor configurations in the CFD modeling. The shear rates in the reactor with baffles were higher especially in the bulk region. Therefore, although the conversion was lower in the reactor with baffles, the higher shear in the bulk region resulted in slightly higher particle size that was due to the agglomeration and coalescence of nucleated polymer particles. In other words, the emulsion polymerization mixture was sensitive to the shear produced especially in the bulk region. 


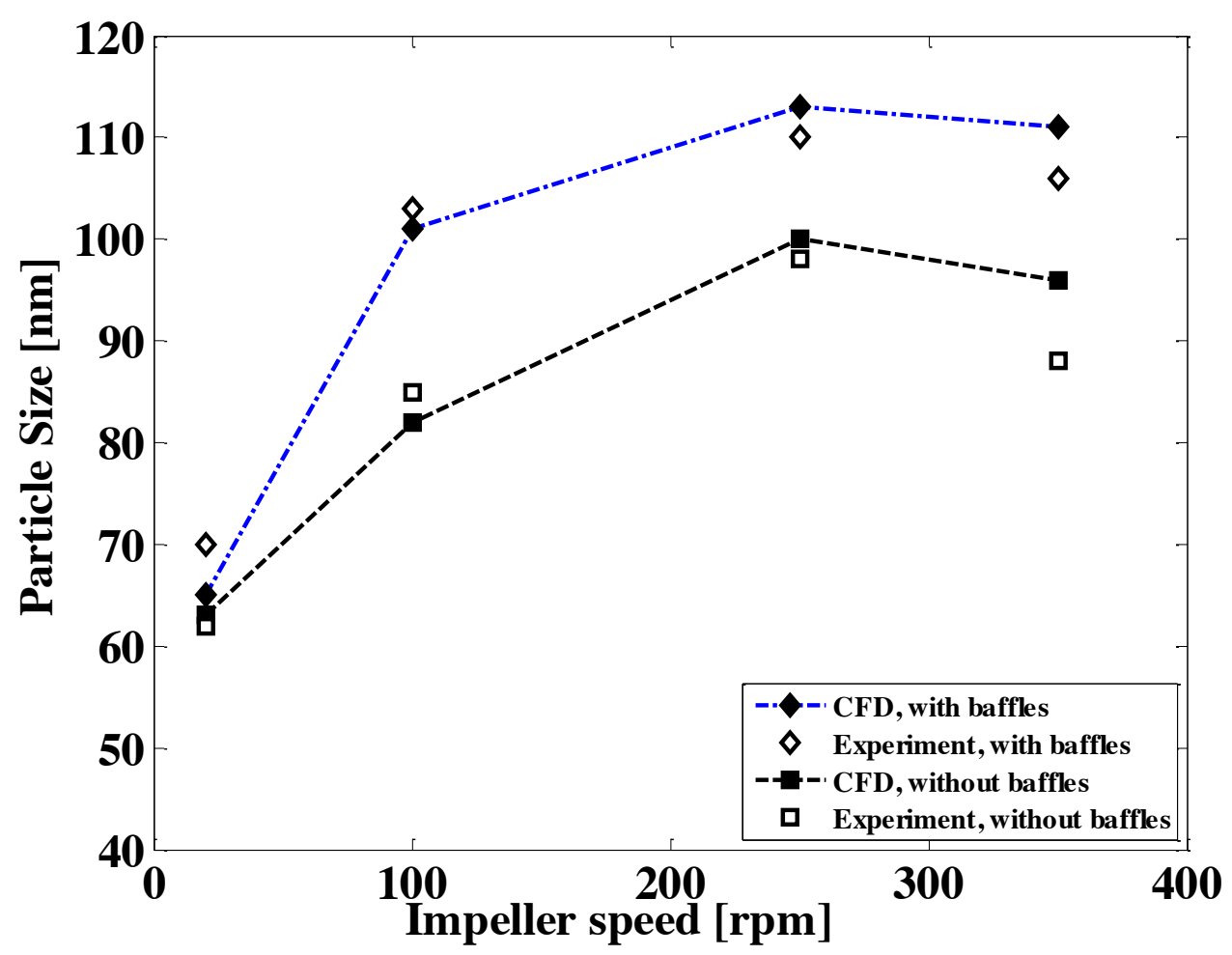

Figure 5.3-5 Sauter mean particle size versus impeller speed at the reaction time of 130 min for the pitched blade impeller and the reactors with baffles and without baffles.

Table 5.3-1 Volume average shear rates computed using the CFD model for the different reactor configurations.

Volume average shear rate $(1 / \mathrm{s})$

\begin{tabular}{c|c|c|c|c|c|c|c|c}
\hline \multirow{2}{*}{$\begin{array}{c}\text { Impeller } \\
\text { speed }\end{array}$} & \multicolumn{2}{|c|}{$20 \mathrm{rpm}$} & \multicolumn{2}{c|}{$100 \mathrm{rpm}$} & \multicolumn{2}{c}{$250 \mathrm{rpm}$} & \multicolumn{2}{c}{$350 \mathrm{rpm}$} \\
\cline { 2 - 8 } & $\begin{array}{c}\text { Impeller } \\
\text { region }\end{array}$ & Bulk region & $\begin{array}{c}\text { Impeller } \\
\text { region } \\
\text { configuration }\end{array}$ & Bulk region & $\begin{array}{c}\text { Impeller } \\
\text { region }\end{array}$ & $\begin{array}{c}\text { Bulk } \\
\text { region }\end{array}$ & $\begin{array}{c}\text { Impeller } \\
\text { region }\end{array}$ & Bulk region \\
\hline $\begin{array}{c}\text { Pitched blade } \\
\text { impeller with baffles }\end{array}$ & $5.3 \times 10^{-4}$ & $6.0 \times 10^{-5}$ & $2.6 \times 10^{-2}$ & $1.8 \times 10^{-3}$ & $1.9 \times 10^{-1}$ & $1.2 \times 10^{-1}$ & $2.6 \times 10^{-1}$ & $1.8 \times 10^{-1}$ \\
\hline $\begin{array}{c}\text { Pitched blade } \\
\text { impeller without } \\
\text { baffles }\end{array}$ & $2.2 \times 10^{-4}$ & $1.0 \times 10^{-5}$ & $8.5 \times 10^{-3}$ & $9.8 \times 10^{-4}$ & $1.6 \times 10^{-1}$ & $9.1 \times 10^{-2}$ & $2.4 \times 10^{-1}$ & $1.5 \times 10^{-1}$ \\
\hline $\begin{array}{c}\text { Rushton impeller } \\
\text { with baffles }\end{array}$ & {$[-]$} & {$[-]$} & $1.5 \times 10^{-1}$ & $2.6 \times 10^{-4}$ & $3.4 \times 10^{-1}$ & $7.8 \times 10^{-3}$ & $5.6 \times 10^{-1}$ & $1.23 \times 10^{-2}$ \\
\hline
\end{tabular}


a)

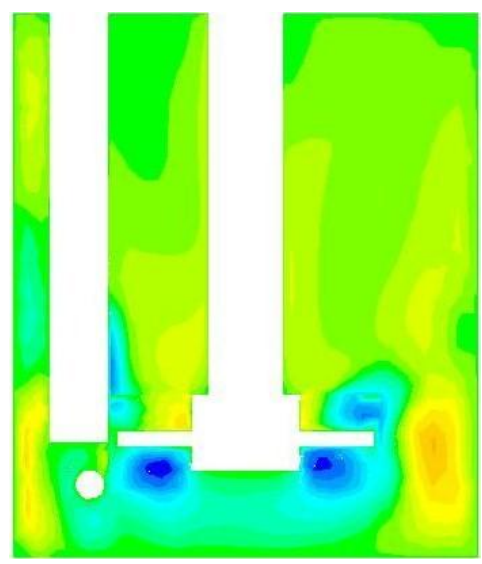

\section{Impeller Region}

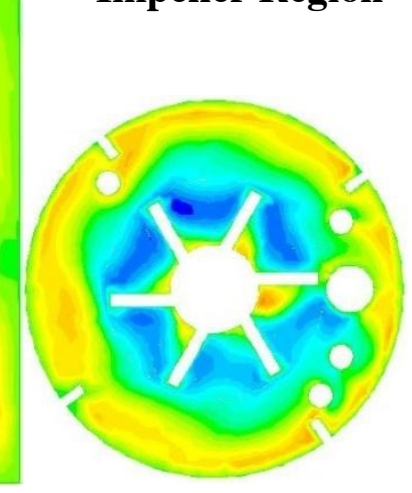

b)
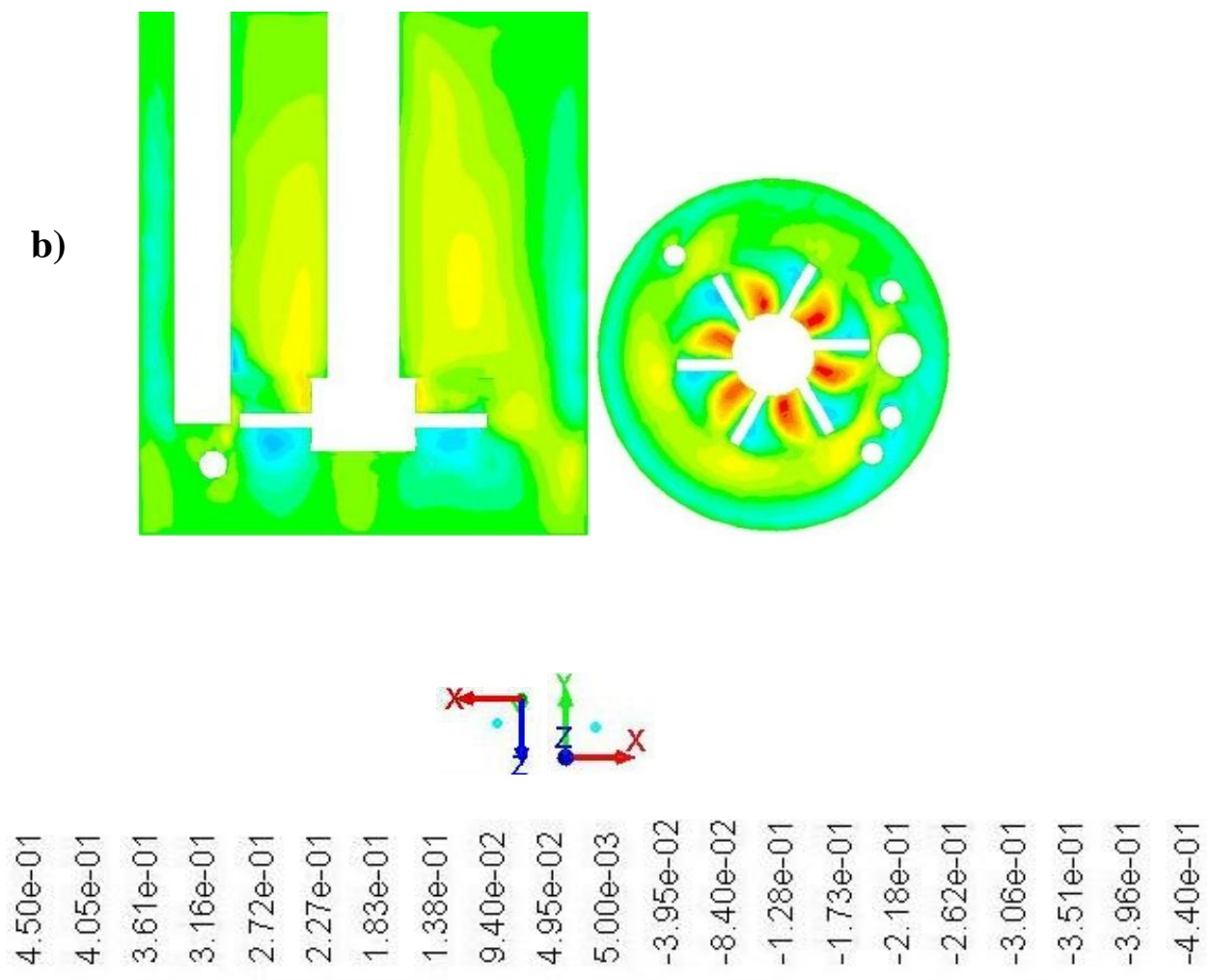

$m / s$

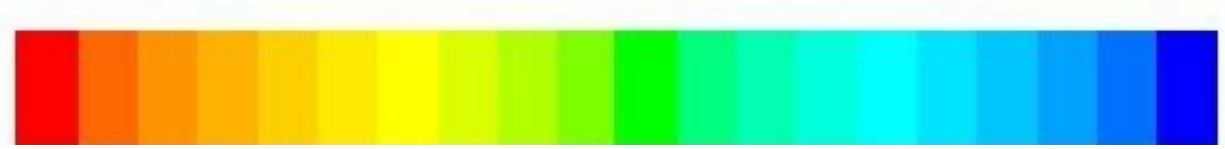

Figure 5.3-6 Contours of axial velocity of mixture $(\mathrm{m} / \mathrm{s})$ generated by the pitched blade impeller at the impeller speed of $350 \mathrm{rpm}$ in the reactor a) with baffles and b) without baffles. 
Effect of the impeller type:

In the second part of the discussion, the effect of impeller type is studied on the conversion, and number density distribution and mean particle size. For better elucidation of the results, the volume average shear rates in the impeller and bulk region and also the flow numbers are compared for the two types of impellers used in this study.

Generally, the radial fluid flow produced by the Rushton impeller mostly circulates into the region above and below the impeller (Paul et al., 2004). The re-circulated fluid mixture then slowly returns to the impeller zone. In contrast, the axial fluid flow generated by the pitched blade produces a strong pumping action and a relatively rapid return to the impeller zone. This affects the flow number which measures impeller ability to generate convective flow. The higher the flow number, the higher the connective flow generated by the impeller (Pakzad et al., 2008; Saeed et al., 2008).

Flow number is obtained using this equation:

$$
F l=\frac{Q}{N D^{3}}
$$

where $Q$ is the impeller pumping flow rate, $N$ is the impeller speed, and $D$ is the impeller diameter. The flow numbers were obtained through the CFD model for the Rushton impeller using the radial velocity profiles calculated at an area surrounding the impeller with a height equal to that of the impeller blade and a diameter equal to the impeller diameter at the blade tip (Pakzad et al., 2008) and for the pitched blade impeller using the axial velocity profiles calculated at a plane exactly below the impeller blades (Saeed et al., 2008). The flow numbers calculated using the CFD model for the pitched blade and Rushton impeller are listed in Table 5.3-2. The calculated flow numbers were smaller for the Rushton impeller compared to the 
pitched blade at different impeller speeds. The convective flow generated as a result of a higher flow number improves the reactant to react and thus the rate of polymerization is enhanced.

Table 5.3-2 Flow numbers computed using the CFD model for the pitched blade and the Rushton impellers at different impeller speeds in the reactors equipped with the baffles.

\begin{tabular}{|l|cc|}
\hline Impeller type & Impeller speed (rpm) & Flow number \\
\hline \multirow{3}{*}{ Pitched blade impeller } & 100 & 0.654 \\
& 250 & 0.663 \\
& 350 & 0.688 \\
Rusthon impeller & 100 & 0.615 \\
& 250 & 0.621 \\
& 350 & 0.627 \\
\hline
\end{tabular}

Figure 5.3-7 compares the profiles of monomer conversion as a function of the impeller speed at reaction time of $130 \mathrm{~min}$ for the pitched blade and Rushton impellers at $60^{\circ} \mathrm{C}$ for the reactors equipped with baffles. Experimental data are presented by white symbols and the CFD predictions by black symbols. The trends of the CFD simulations are shown using the dotted lines. As can be seen in Fig. 9, with the elevation of the Rushton impeller speed from $100 \mathrm{rpm}$ to $250 \mathrm{rpm}$ and then to $350 \mathrm{rpm}$, the conversion was increased and no reduction in conversion at high agitation rates was observed. In other words, replacement of the pitched blade impeller with the Rushton impeller increased the conversion obtained by experiments by about $6 \%$ at $100 \mathrm{rpm}$ and $2 \%$ at $350 \mathrm{rpm}$, but resulted in a lower conversion of about $10 \%$ at $250 \mathrm{rpm}$. According to the CFD results, the installation of the Rushton impeller increased the conversion by about $8 \%$ at $100 \mathrm{rpm}$ and $2 \%$ at $350 \mathrm{rpm}$, and reduced the conversion by about $16 \%$ at $250 \mathrm{rpm}$.

According to the literature (Paul et al., 2004), the Rushton impeller creates fluid flow directed radially outward of the impeller and produces two circulating loops one below and one above the 
impeller. Mixing occurs between the loops but less intensely within each loop. The volume average shear rates listed in Table 3 reveals that the shear rates obtained in the reactor equipped with the Rushton turbine were higher in the impeller region but smaller in the bulk region compared to the pitched blade impeller at different agitation rates.

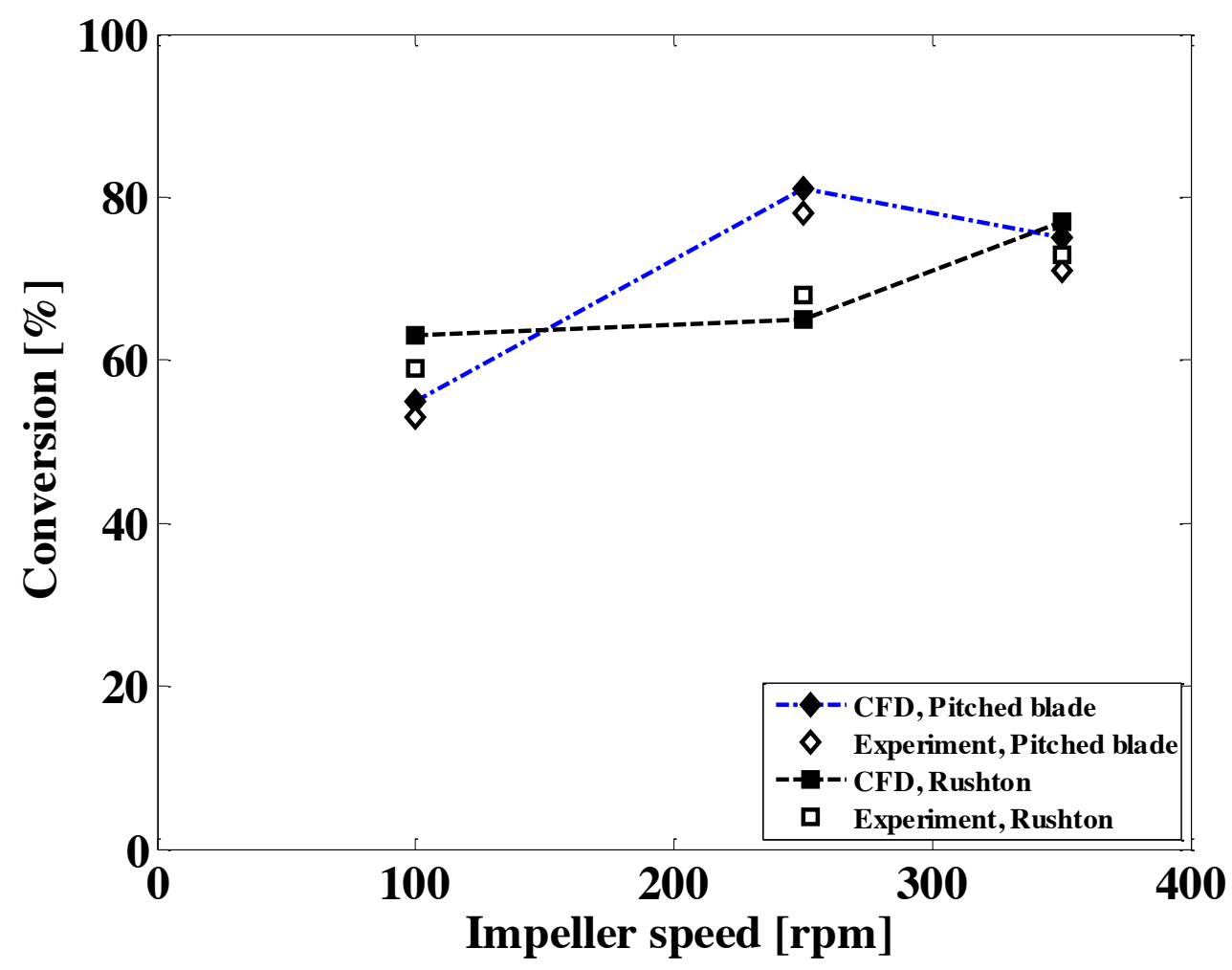

Figure 5.3-7 Monomer conversion versus impeller speed at the reaction time of 130 min for the pitched blade and the Rushton impellers in the reactor with baffles.

At the lower impeller speeds, i.e. $100 \mathrm{rpm}$, the shear rate in the Rushton impeller region resulted in better mixing and collision of reactants and diffusion of monomer to micelles, which resulted in a higher conversion compared to that obtained using the pitched blade impeller. However, by increasing the impeller speed to $250 \mathrm{rpm}$, although the shear was higher in the impeller region when the Rushton was used, yet the enhanced pumping action of the pitched blade resulted in a 
higher conversion compared to the Rushton impeller. As mentioned earlier with the use of the pitched blade impeller, further increase in the agitation speed up to $350 \mathrm{rpm}$, resulted in the instability of emulsion due to a higher shear rate in the bulk region, which led to the reduction in conversion. However, the improved mixing in the Rushton impeller region and a lower shear rate in the bulk region produced a slightly higher conversion value at $350 \mathrm{rpm}$.

The number densities obtained using the Rushton impeller is shown in Figure 5.3-8. The values at the impeller speeds of 100 to $350 \mathrm{rpm}$ were higher compared to the number densities calculated in the reactor equipped with the pitched blade impeller (Figure 5.3-3). Monomer conversion was higher at the speed of 100 and $350 \mathrm{rpm}$ when the Rushton impeller was used compared to the pitched blade impeller. Therefore, the nucleation was higher at these two agitation rates and resulted in the higher number densities. Besides, at $250 \mathrm{rpm}$, although the conversion was higher with the use of the pitched blade impeller rather than the Rushton impeller, enhanced values of number density was obtained. The reason can be due to the smaller particle sizes formed when the Rushton impeller was utilized at $250 \mathrm{rpm}$. In other words, as a result of the lower shear rates in the bulk region at the different impeller speeds (Table 5.3-1), the agglomeration of particles were limited and thus the higher number densities were obtained with the use of Rushton impeller which is being discussed afterwards.

Figure 5.3-9 compares the profiles of the particle size as a function of the impeller speed at the reaction time of $130 \mathrm{~min}$ for the pitched blade and Rushton impellers at $60^{\circ} \mathrm{C}$ in the reactors equipped with baffles. Experimental data are presented by white symbols and the CFD predictions by black symbols. The trends of the CFD simulations are shown by dotted lines. 


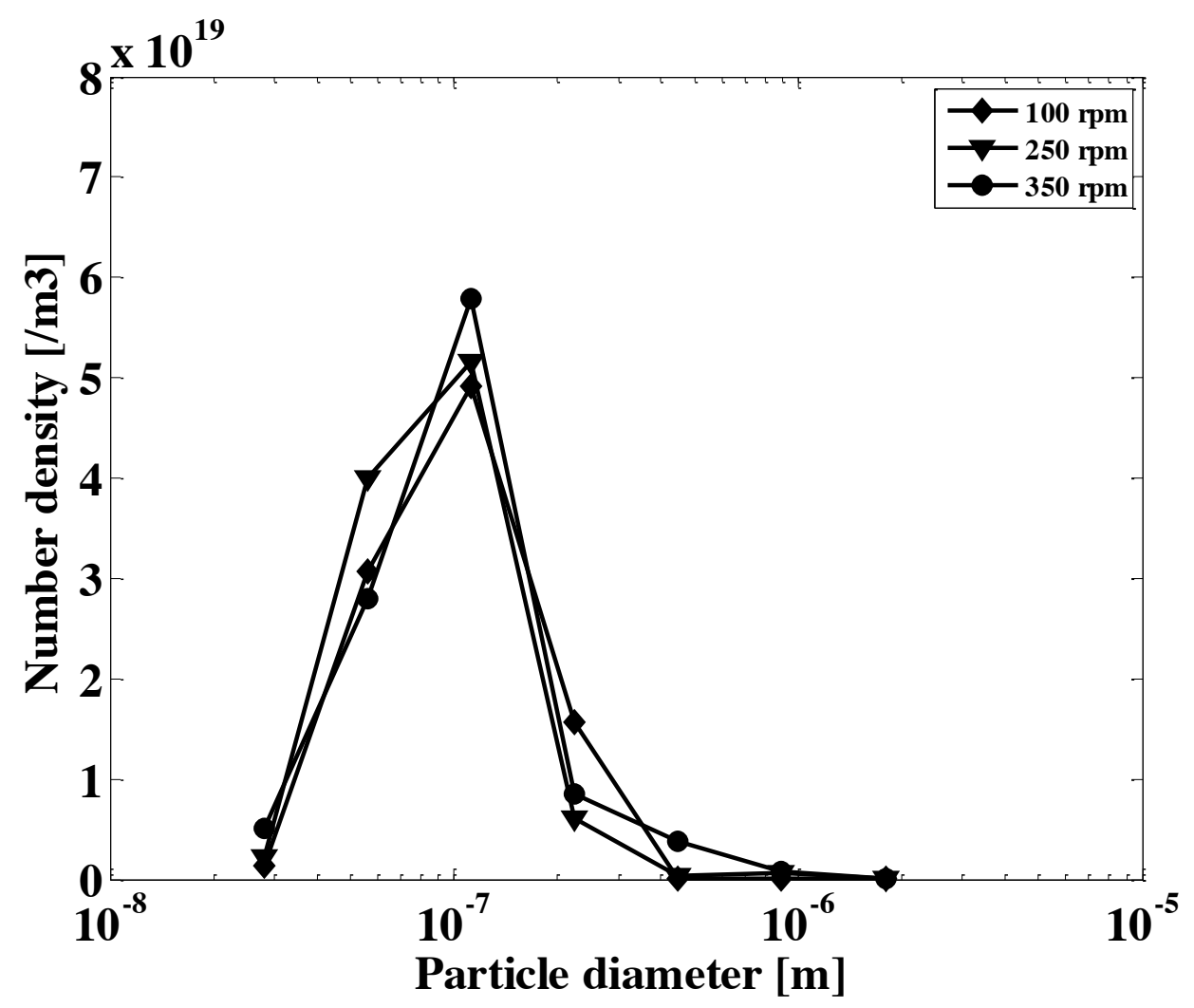

Figure 5.3-8 Number density distriubtion of particles computed through the CFD model for the Rushton impeller at the speed of 100, 250, and $350 \mathrm{rpm}$ in the reactor with baffles.

The particle size values obtained through both experiment and CFD modeling for the Rushton impeller were smaller compared to those achieved for the pitched blade impeller since the number densities were higher with the Rushton impeller at all impeller speeds. At the impeller speeds of $100 \mathrm{rpm}$ and $350 \mathrm{rpm}$, the conversion was higher with the use of the Rushton compared to the pitched blade impeller. Therefore, the available un-reacted monomer was limited for the growth of particles and consequently the particle sizes were reduced with the use of the Rushton impeller as shown in Figure 5.3-9. 


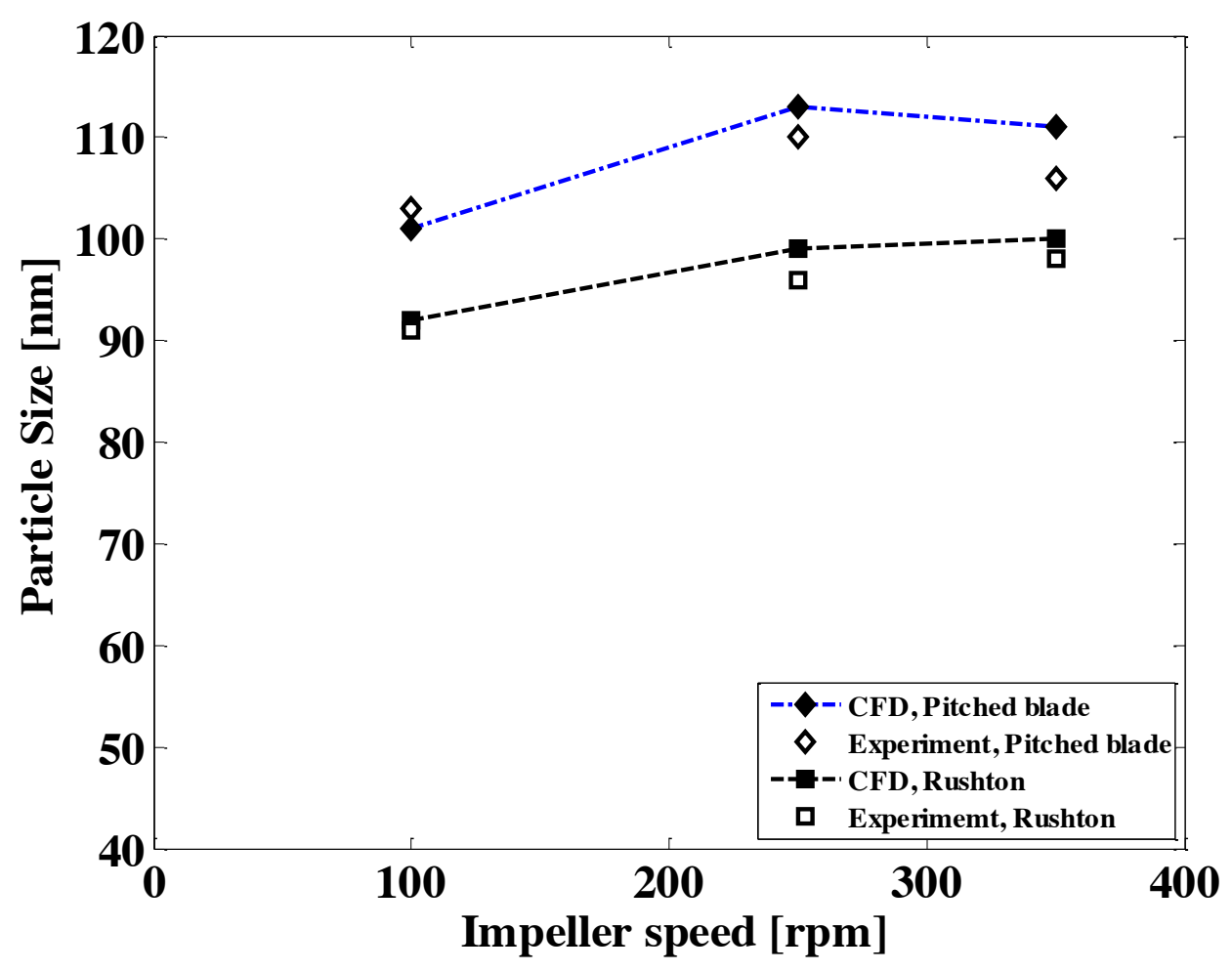

Figure 5.3-9 Sauter mean particle size versus impeller speed at the reaction time of 130 min for the pitched blade and the Rushton impellers in the reactor with baffles.

Besides, at all the impeller speeds of $100-350 \mathrm{rpm}$, the smaller particle size obtained in the reactor equipped with the Rushton impeller was as a result of the slighter agglomeration of polymer particles due to a smaller average shear rate in the bulk region compared to the values achieved by using the pitched blade impeller according to Table 5.3-1.

Finally, Figure 5.3-10 depicts the contours of polymer volume fraction in the reactor equipped with the pitched blade and Rushton impellers at the speed of $250 \mathrm{rpm}$. The polymer volume fraction was better distributed within the tank domain when the pitched blade was used due to improved flow capacity and recirculation of reagents in the reaction mixture. The higher flow number for the pitched blade impeller compared to that for the Rushton impeller (see Table 5.32) confirms this presumption. 
The novelty of this research in the aspect of CFD modeling was incorporation of chemical reaction kinetics to the population balance through nucleation and growth rates while including the velocity gradients in the mixing emulsion polymerization reactor tank domain.

In this study detail of mixing such as shear rate in the impeller/bulk region, flow number, particle size, conversion and number density within the reaction mixture was studied.

The information of this research can be used for the progress the emulsion polymerization process in industrial application such as scale up and reactor design. Furthermore, the effect of design parameters and operating conditions on the performance of emulsion polymerization systems and particle nucleation and growth can be used to determine the heat and mass transfer rates.

\subsubsection{Concluding Remarks}

A novel CFD model was developed in this study to explore the effect of mixing on emulsion polymerization of MMA. In this model, the chemical reaction kinetics, population balance through nucleation and growth rates, and the velocity gradients generated by the impeller rotation were coupled together. The reactor model was comprised of continuity equation, three momentum equations for the velocity components for the mixture of two phases, two $k$ and $\varepsilon$ turbulence equations, one species continuity equation for the monomer specie in the primary phase, seven equations for the bin fractions of the secondary phase and the continuity volume fraction equation for the secondary phase. The simultaneous solution approach was employed instead of the sequential approach to enhance the accuracy of the simulation results. In order to calculate the particle size distribution, rather than obtaining only the mean diameter, the parallel computation with 36 dual cores was utilized to reduce the extensive computational time and cost. 
a)

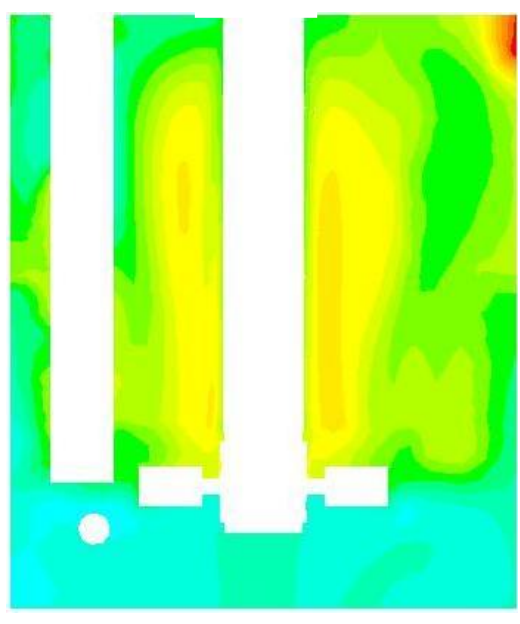

b)

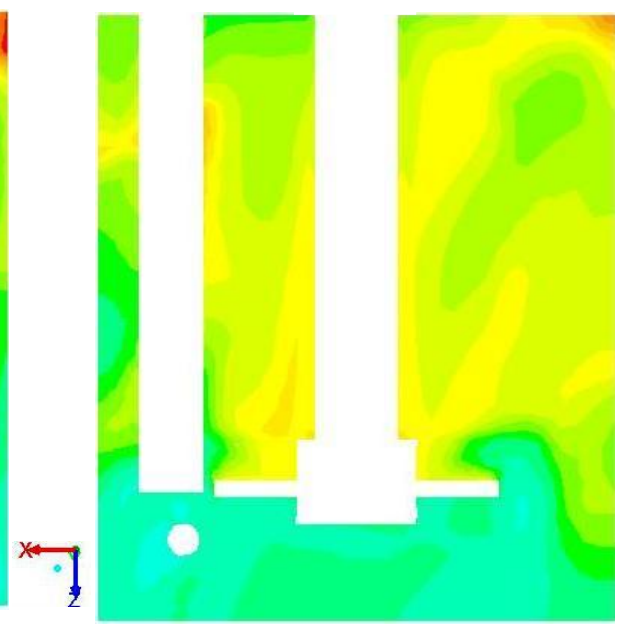

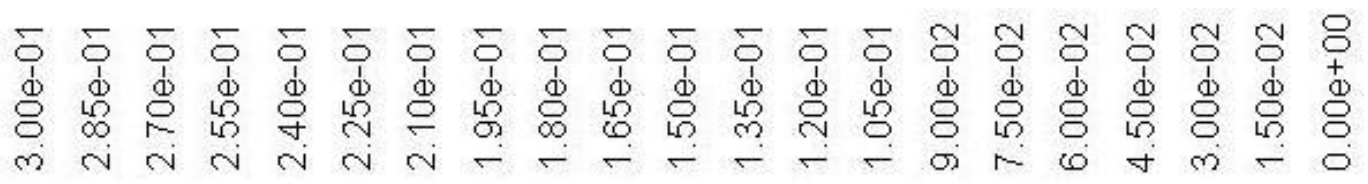

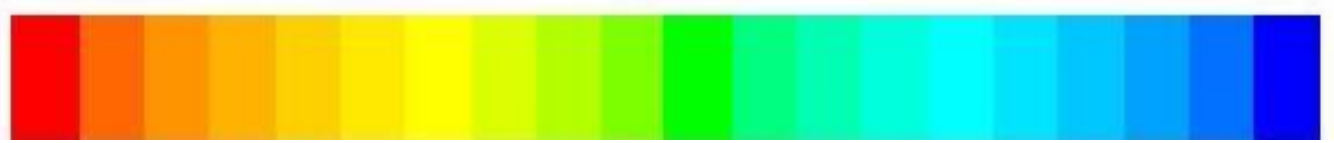

Figure 5.3-10 Contours of the second phase volume fraction in the reactor equipped with the baffles at the speed of $250 \mathrm{rpm}$ for the: a) Rushton impeller and b) pitched blade impeller.

To validate the CFD model, the computed monomer conversion, the mean particle size, and the number density for different impeller speeds and impeller types in the reactors with and without baffles were compared successfully to the experimental data.

The validated CFD model was then employed to investigate the effects of the impeller speed, impeller type, and baffles on MMA conversion, particle size, and number density distribution of polymer particles. With the use of the pitched blade impeller, the vigorous stirring at $350 \mathrm{rpm}$ induced instability in emulsion by excessive shear rate on the emulsion mass. Besides, the conversion was decreased when the baffles were installed in the reactor due to the accumulation 
of monomer droplets behind the baffles. The particle sizes generated in the reactor equipped with the baffles were larger due to the higher shear rates in the bulk region compared to those obtained in the reactor without the baffles.

At the lower impeller speeds, i.e. $100 \mathrm{rpm}$, the shear rate in the Rushton impeller region resulted in better mixing and collision of reactants and diffusion of monomer to micelles, which resulted in a higher conversion compared to that obtained using the pitched blade impeller. However, by increasing the impeller speed to $250 \mathrm{rpm}$, although the shear was higher in the impeller region when the Rushton was used, yet the enhanced pumping action of the the pitched blade resulted in a higher conversion compared to the Rushton impeller. However, the improved mixing in the Rushton impeller region and a lower shear rate in the bulk region produced a slightly higher conversion value at $350 \mathrm{rpm}$.

The number densities of particles were higher when the baffles were removed. Furthermore, he number densities achieved using the Rushton impeller were higher compared to those for the pitched blade impeller in the reactor with the baffles. Finally, the distribution of polymer volume fraction within the reactor equipped with the pitched blade impeller was more uniform than that for the Rushton impeller due to a better circulation and higher flow number.

The novel CFD model developed in this study enables us to explore the effects of the design parameters and operating conditions on emulsion polymerization. This information can be used to optimize the design of the reactors utilized for emulsion polymerization. The target of future work is to incorporate the break up and coalescence phenomena in the CFD modeling to elucidate the particle size changes. 


\subsection{CFD modeling of the mixing of water in oil Emulsions (Fathi Roudsari et al., 2012)}

\subsubsection{Introduction}

Quite a number of studies have focused on the modeling of the droplet size distribution in an emulsion of water and oil. Nevertheless, the prediction of the particle sizes in an inhomogeneous flow, where the flow velocity is changing spatially in magnitude and direction and with time, is not yet well recognized. In this study, the development of a CFD model, based on the population balance for a water-in-oil emulsion system is discussed. All the equations are described in Chapter 4 (Equation 4.2-1 to Equation 4.2-17). The model was validated with the experimental data reported in the literature (Boxall et al., 2010). The validated model was then employed to explore the effect of the impeller speed, oil viscosity, and volume fraction of water on the cumulative probability size distribution, number density, and distribution of water volume fraction. The schematic diagram of the emulsion cell and the geometry grid are shown in Figures 4.2-1 and 4.2-2.

Since the population balance equations for all bins (classes) were solved simultaneously with transport equations for two phases along with the turbulence equations for $k$ and $\varepsilon$, the number of bins (classes) was limited to 7 and satisfactory results were obtained. According to literature, sufficient number of classes should be used to minimize the discretization errors (Laakkonen et al., 2007). Table 4.2-2 in chapter 4 shows the diameter of the drop sizes used in discrete model. To validate the CFD model, the computed droplet size distributions at different impeller speeds were compared to the experimental data reported by Boxall et al. (2010). 


\subsubsection{Results and discussion}

Generally, according to literature (Stamatoudis and Tavlarides, 2007, Vankova et al., 2007), for the emulsification process in turbulent flow, two different regimes of emulsification should be specified, which are termed "turbulent inertial" and "turbulent viscous" regimes, respectively. In the turbulent inertial regime, the drops are larger in diameter than the smallest turbulent eddies in the continuous phase, whereas in the turbulent viscous regime the drop diameter is smaller than the size of the smallest eddies. Stamatoudis and Tavlarides (2007) reported that for a system of low continuous phase viscosity or high rotational speed, turbulent eddies are predominant in the vessel and provide the energy required for breakage. However, as the viscosity of the continuous phase is increased and the rotational speed is decreased, the turbulent eddies diminish, and shear forces become more important.

Figure 5.4-1 depicts the cumulative probability drop size distribution of $15 \mathrm{v} / \mathrm{v} \%$ water in Conroe oil emulsion as a function of impeller speed. It can be seen that as the impeller speed was increased, the water droplet size was reduced from the range of $85-280 \mu \mathrm{m}$ at $300 \mathrm{rpm}$ to the range of 50-210 $\mu \mathrm{m}$ at $400 \mathrm{rpm}$ and $25-130 \mu \mathrm{m}$ at $600 \mathrm{rpm}$, respectively.

In fact, the drop breakage is the prevailing process at the higher impeller speed. Under these conditions, an increase in the external inertial stress arising from the turbulent pressure fluctuations leads to a destabilization of the drops, consequently, the maximum drop size reduces and the entire drop size distribution shifts towards the smaller drop sizes. The similar decrease in particle size was observed by Ruiz et al. (2002) when increasing the impeller speed. Since Conroe oil (continuous phase) had a relative low viscosity $(3.1 \mathrm{cP})$, the turbulent eddies were able to generate sufficient energy for breaking up the water droplets. When the impeller speed 


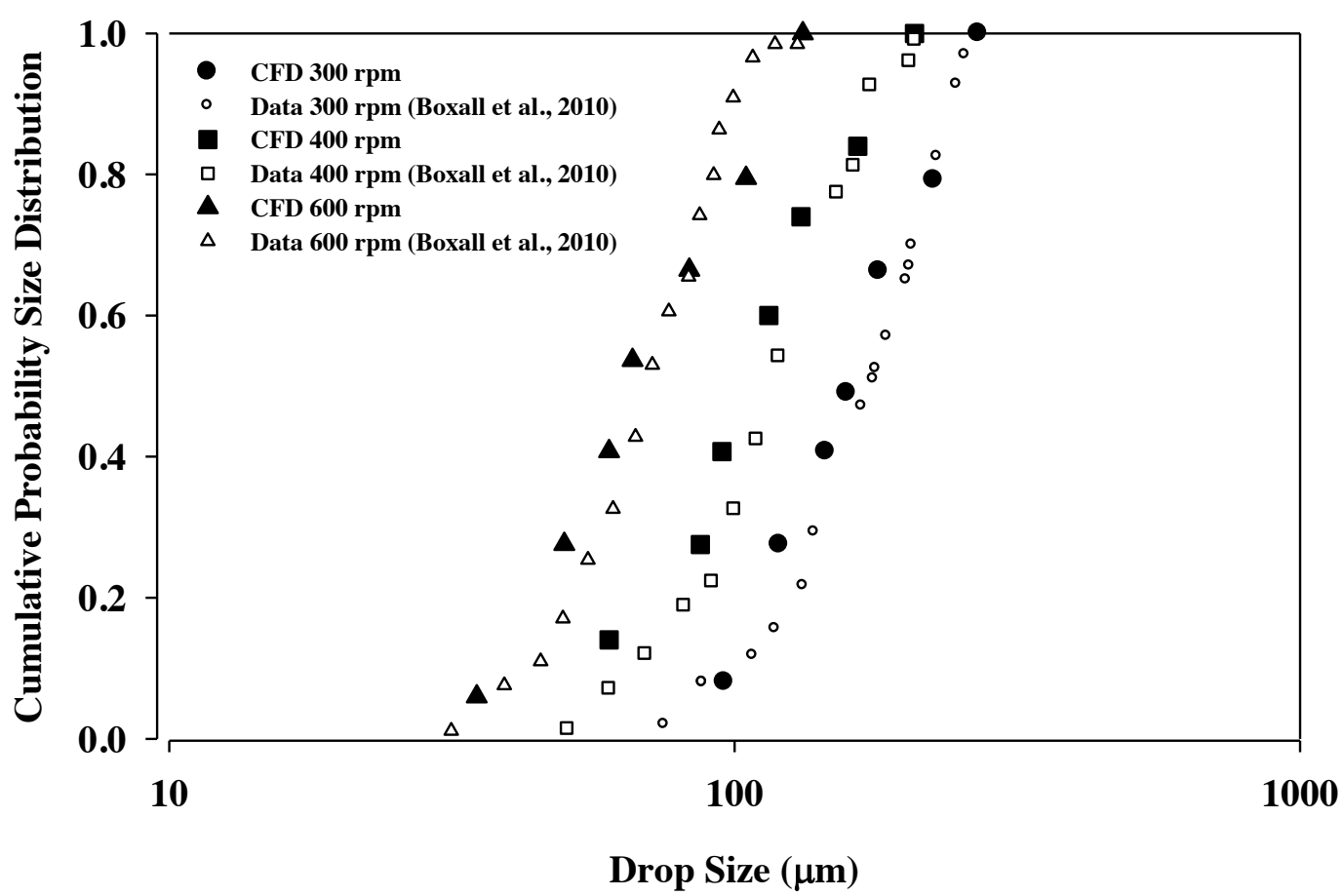

Figure 5.4-1 Cumulative probability drop size distribution versus droplet diameter at impeller speeds of: • 300, 400 and $\boldsymbol{\Delta} 600 \mathrm{rpm}$ and continuous phase of Conroe oil with $15 \mathrm{vol} \%$ water at $20^{\circ} \mathrm{C}$.

was increased from $300 \mathrm{rpm}$ to $400 \mathrm{rpm}$ and then to $600 \mathrm{rpm}$, more eddies were produced and the size of droplets diminished due to breakage. According to literature (Luo and Svendsen, 1996; Narsimhan et al., 1979), viscous forces can be neglected when the size of eddies generated by turbulent flow are smaller than the size of droplets. These small eddies create high-frequency oscillations in droplets resulting in droplet breakage. The logarithm of number density of droplets at different impeller speeds is demonstrated in Figure 5.4-2. The logarithm of the number density was shifted towards smaller droplet size as the impeller speed was increased from $300 \mathrm{rpm}$ to $400 \mathrm{rpm}$ and then to $600 \mathrm{rpm}$. Since the discrete method was employed to solve the population balance equations, the size range in $\mu m$ for each simulation was predefined considering the experimental data (Boxall et al., 2010). 
The number density presents the number of droplets in each bin in water phase per unit volume of the tank and in this study the logarithm of the number density was plotted versus the drop size for each simulation. Regarding the high values of number density, it must be mentioned that these values were not unexpected as by a simple approximation of a pre-assumed bin fraction, and considering the known second phase volume fraction and tank volume, initial number density, can be obtained and compared to the CFD calculated values. In order to validate the CFD predictions, we also calculated the cumulative size distribution (Milton and Arnold, 1990) of number densities and compared them to those reported in the literature by Boxall et al. (2010). The importance of breakage and coalescence depends on the size of droplets, turbulent intensity, and the physical properties of the system. Maggioris et al. (1998 and 2000) showed that the breakage predominates in the regions with higher turbulent intensity (e.g. around the impeller). This can be explained in terms of the increased turbulent kinetic energy imparted to the drops, thus resulting in a higher breakage frequency (Maggiroris et al., 1998; Ok et al., 2003; Ruiz et al., 2002; Takahashi and Takeuchi, 1992). Besides, the effect of coalescence should be taken into account in reduction of size distribution at higher stirring speeds.

Figures 5.4-2a, b, and c depict a decrease in the number density for the first six drop sizes and then an increase in the number density for the largest drop size. This shows the tendency of the smaller drops to coalescence within the specified droplet sizes. Generally, the coalescence rate is a function of the collision rate between the drops and the coalescence efficiency between colliding drops (Luo, 1993; Maggiroris et al., 2000; Shinnar, 1961; Shinnar and Church, 1960; Stamatoudis and Tavlarides, 2007). 
a)

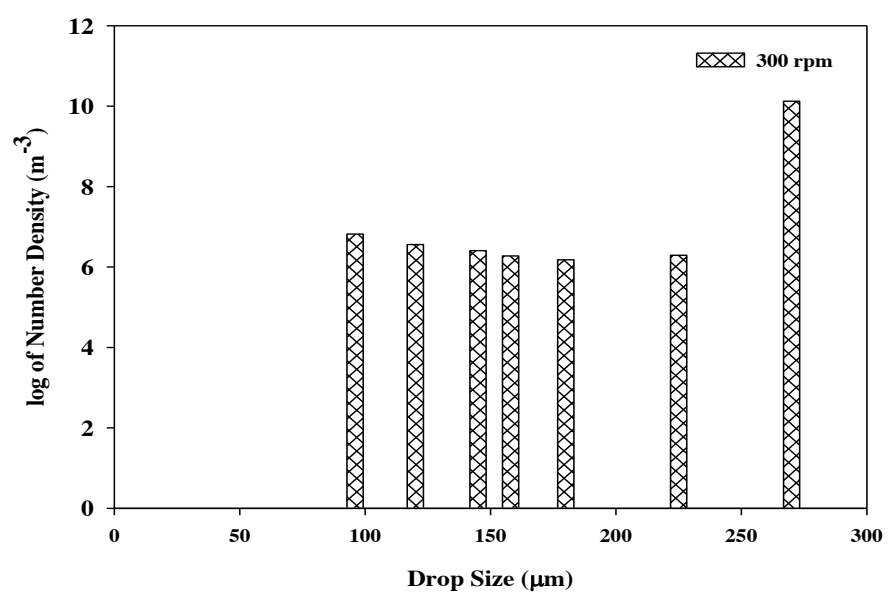

b)

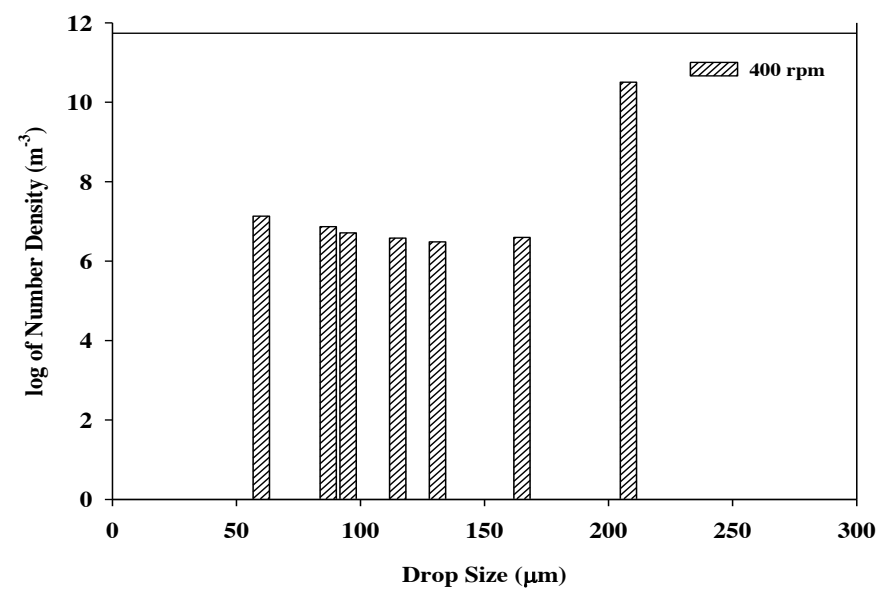

c)

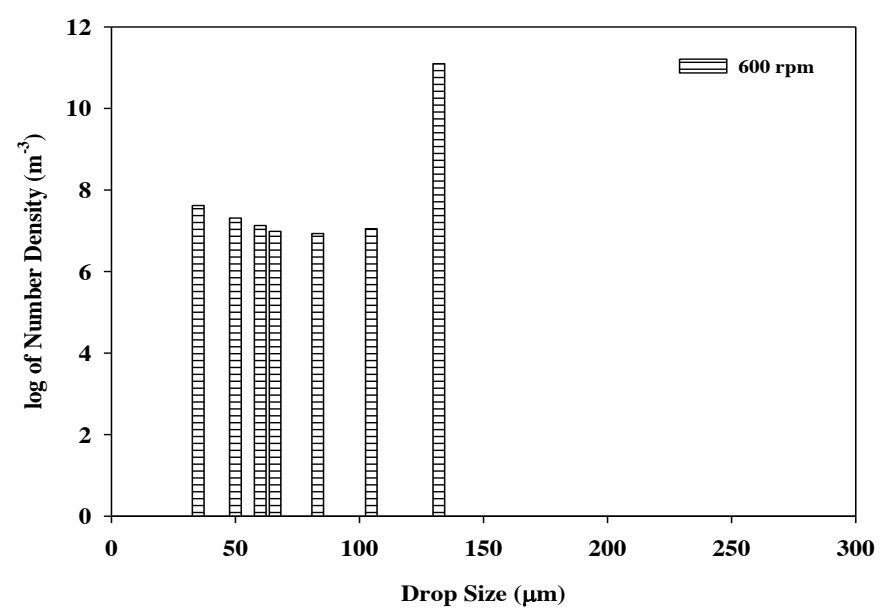

Figure 5.4-2 Logarithm of number density of droplets at impeller speed of a) $300 \mathrm{rpm}$, b) 400 rpm and c) $600 \mathrm{rpm}$ in Conroe oil and $15 \mathrm{vol} \%$ water at $20^{\circ} \mathrm{C}$. 
The collision rate depends on the drop size and on the hydrodynamics of the dispersion. The coalescence effic a) sends on the time tl b) colliding drops rems c) intact and the time required for draining of the intervening liquid film between them to achieve the film rupture and coalescence. Therefore, in Figures 5.4-2, the reduction in the droplet size at higher impeller speeds i.e. $400 \mathrm{rpm}$ and $600 \mathrm{rpm}$ compared to that at $300 \mathrm{rpm}$ can be explained in terms of the increase in the production of smaller eddies at higher impeller speeds, which enhances the breakage rate, while the adhesion energy, collision rate, and coalescence efficiency between the middle sized bins were predominant, which induced coalescence and raised the population of the largest size droplets. Figure 5.4-3 demonstrates the turbulence kinetic energy of the mixture at impeller speeds of 300, 400 and $600 \mathrm{rpm}$ for Conroe oil as the continuous phase. The turbulence kinetic energy was increased with an increase in impeller speed which led to more droplet breakage and narrower size distribution.
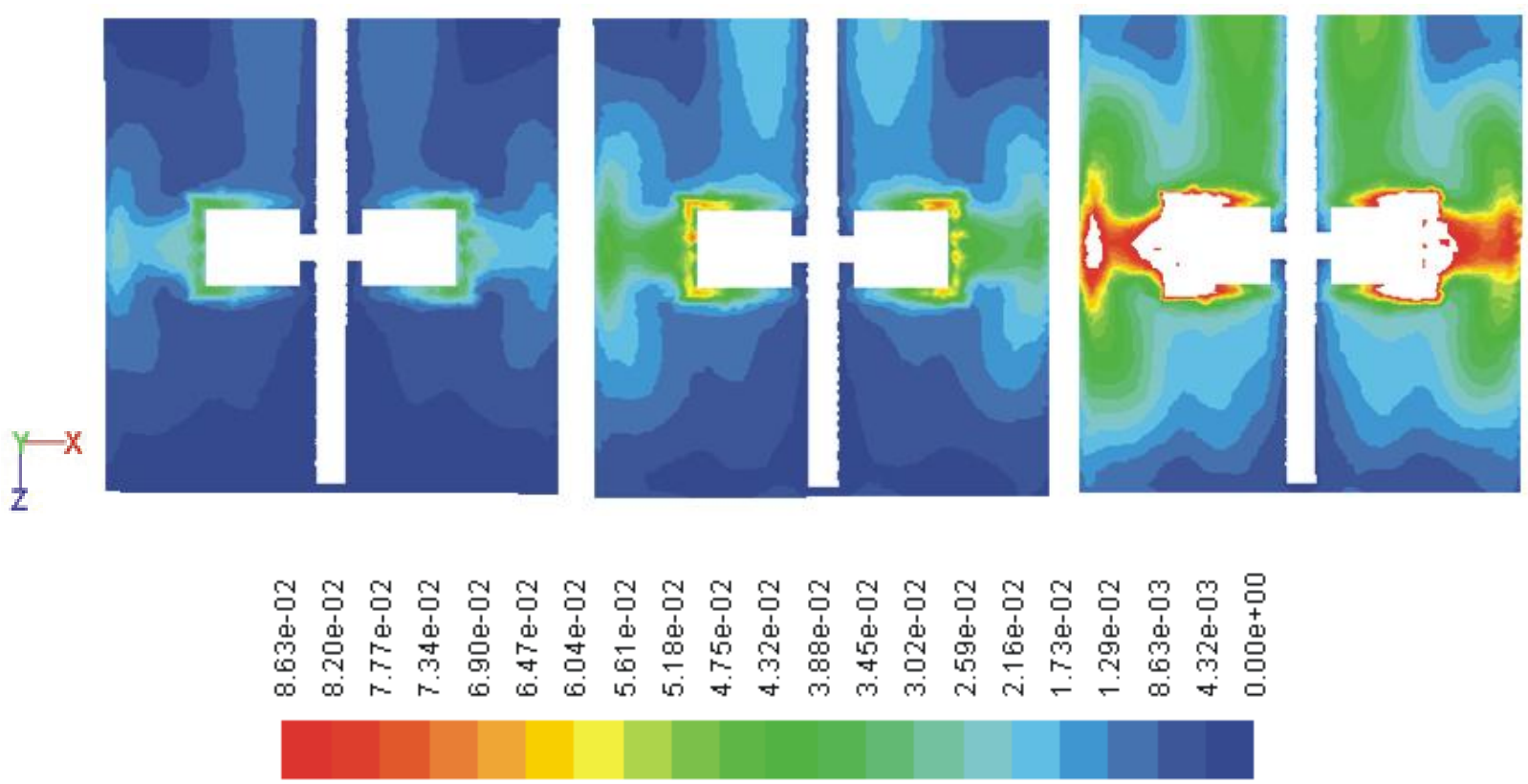

Figure 5.4-3 Contour of turbulent kinetic energy $\left(\mathrm{m}^{2} / \mathrm{s}^{2}\right)$ at different impelle speeds: a) $300 \mathrm{rpm}$, b) $400 \mathrm{rpm}$, and c) $600 \mathrm{rpm}$ in Conroe oil and $15 \mathrm{vol} \%$ water at $20^{\circ} \mathrm{C}$. 
As can be seen in Figure 5.4-4a, the volume fraction of the dispersed phase (water) was high beneath the turbine and low above the impeller at $300 \mathrm{rpm}$. This was due to the higher density of water compared to the density of the continuous phase (oil) and insufficient turbulent energy generated by the impeller to circulate water within the tank. With the increase of the stirring rate to $400 \mathrm{rpm}$ (Figure 5.4-4b) and then to $600 \mathrm{rpm}$ (Figure 5.4-4c), the distribution of second phase became more homogenous in both lower and upper regions of the turbine. This was due to an increase in the velocity magnitude and turbulent kinetic energy throughout the tank which resulted in improved recirculation rate (Maggioris et al., 1998).

a)

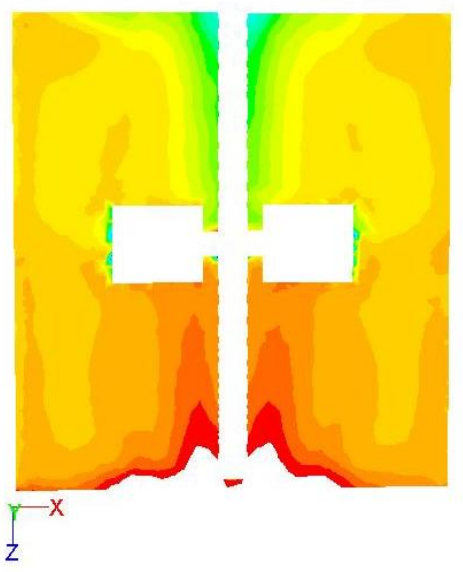

b)

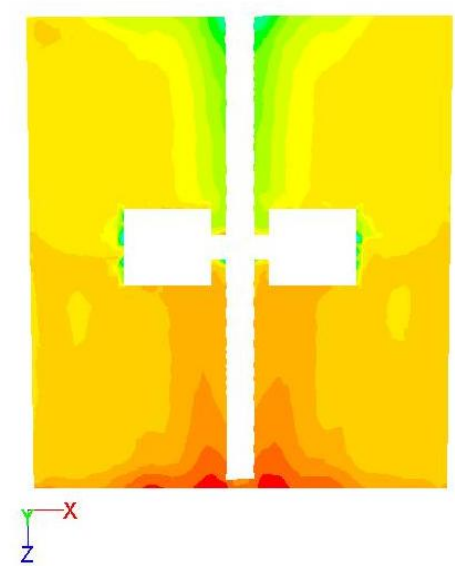

c)

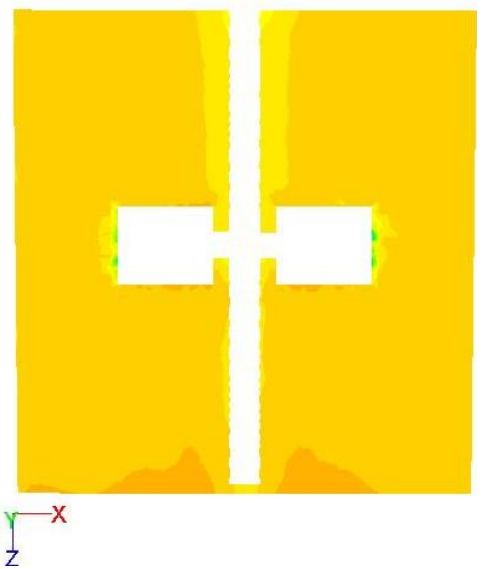

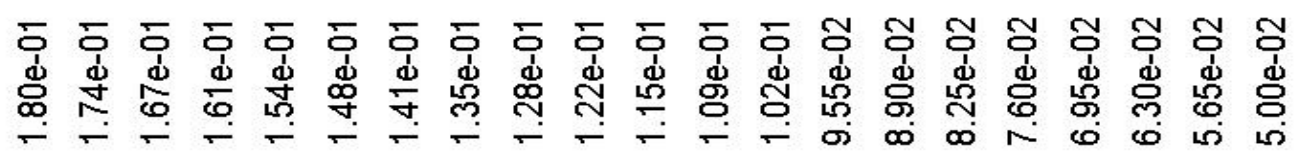

Figure 5.4-4 Contour of water volume fraction at different impeller speeds: a) $300 \mathrm{rpm}$, b) 400 $\mathrm{rpm}$, and c) $600 \mathrm{rpm}$ in Conroe oil and $15 \mathrm{vol} \%$ water fraction at $20^{\circ} \mathrm{C}$. 
As mentioned before, there are two different regimes of emulsification,, which are called turbulent inertial and turbulent viscous regimes (Stamatoudis and Tavlarides, 2007; Vankova et al., 2007). In the turbulent inertial regime, the small turbulent eddies in the disperse phase are predominant, whereas in the turbulent viscous regime the shear forces are responsible for the breakage of droplets. Several studies have been conducted on turbulent inertial regime while investigations on the dispersion in turbulent viscous regime have been rarely reported (Baldyga and Podgórska, 1998; Vankova et al., 2007; Walstra, 1983, 2005). Two types of oil were considered in this study: Conroe oil with a viscosity of $3.1 \mathrm{cP}$ and interfacial tension of 20 $\mathrm{mN} / \mathrm{m}$, and Troika oil with a viscosity of $20 \mathrm{cP}$ and interfacial tension of $11 \mathrm{mN} / \mathrm{m}$ (see Table 4.2-1 in chapter 4). The CFD model was employed to calculate the cumulative probability size distribution and the logarithm of number density of the dispersed phase for both types of oil as continuous phase at the stirring speed of $300 \mathrm{rpm}$ (Figures 5.4-5 and 5.4-6).

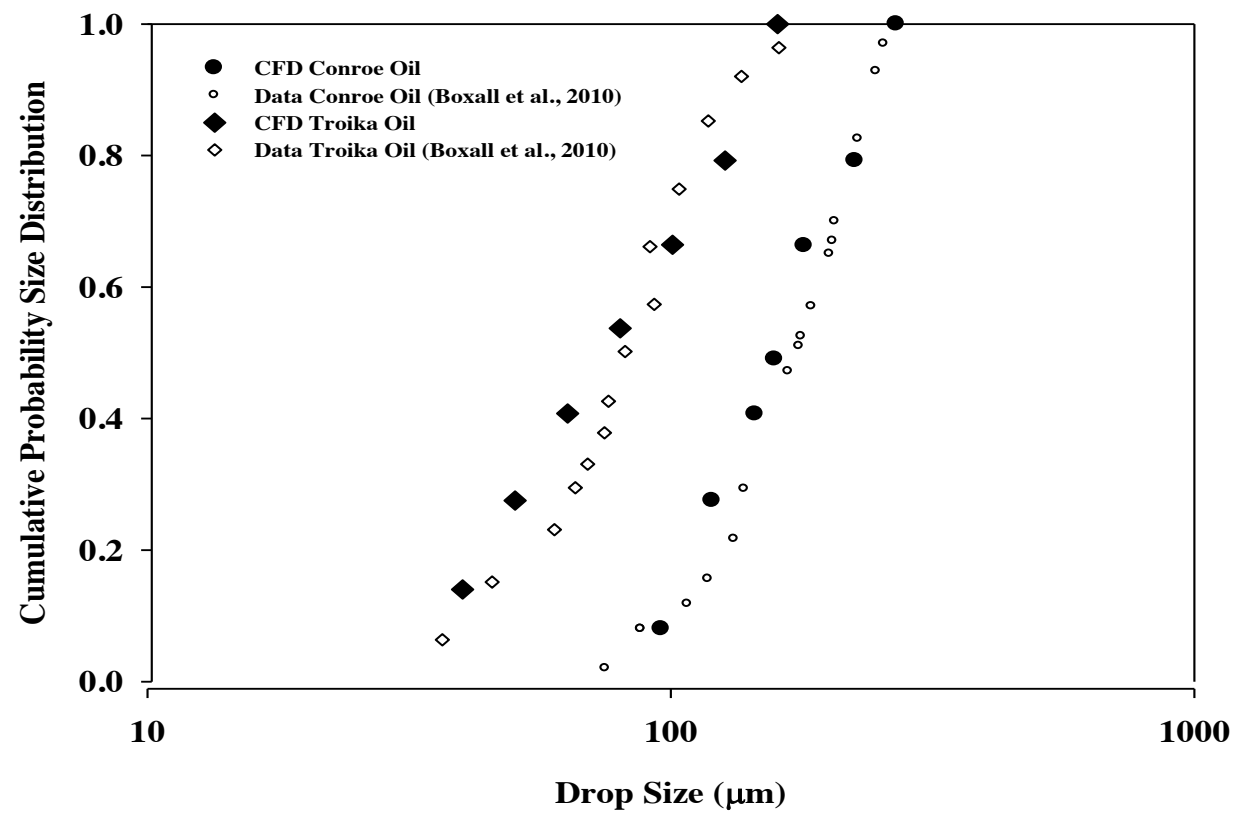

Figure 5.4-5 Effect of the continuous phase type ( Troika oil and $\bullet$ Conroe oil) on the cumulative probability drop size distribution at $300 \mathrm{rpm}$ and $15 \mathrm{vol} \%$ water at $20^{\circ} \mathrm{C}$. 


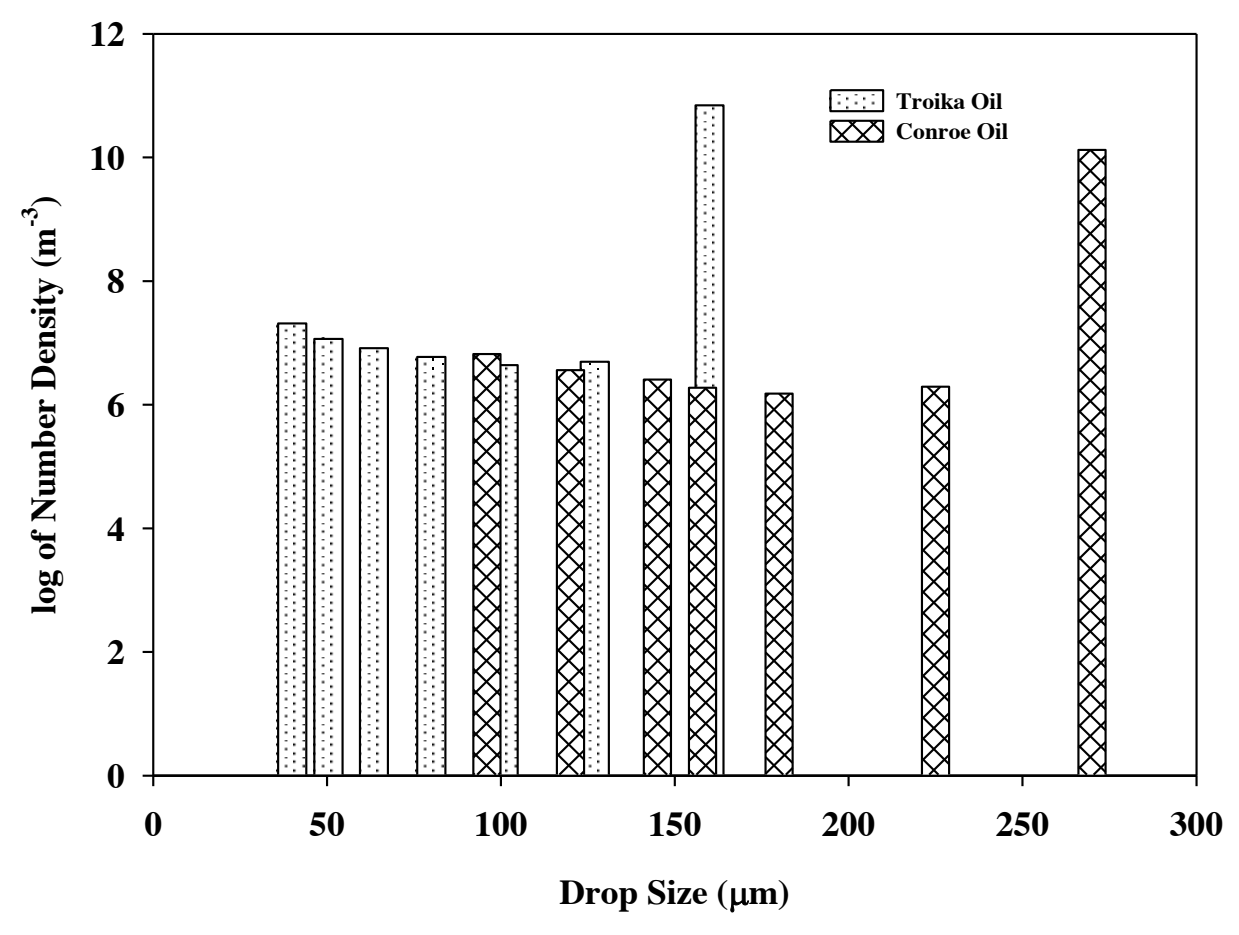

Figure 5.4-6 Effect of the continuous phase type on logarithm of number density of droplets at $300 \mathrm{rpm}$ and $15 \mathrm{vol} \%$ water at $20^{\circ} \mathrm{C}$.

The CFD results were in good agreement with the experimental data reported by Boxall et al. (2010). According to Kolmogorov theory (Maggioris et al., 2000; Vankova et al., 2007), the viscosity increase in continuous phase, leads to increase of the size of the smallest turbulent eddies. As a result, the emulsified drops could become smaller than the size of smallest turbulent eddies and eventually the emulsification would take place in the viscous regime. Thus, the shear forces become more effective in droplet breakage (Stamatoudis and Tavlarides, 2007). Moreover, the gain in the continuous phase viscosity can lead to lower coalescence rate which depends on the collision rate and the coalescence efficiency between drops. As mentioned before, the coalescence efficiency is dependent on the time needed for drainage of the intervening liquid film between droplets so that film rupture and coalescence can occur (Luo, 
1993; Maggiroris et al., 2000; Shinnar, 1961; Shinnar and Church, 1960; Stamatoudis and Tavlarides, 2007). From Figures 5.4-5 and 5.4-6, it can be seen that an increase in continuous phase viscosity caused the droplet size distribution to shift towards the smaller sizes. The water droplet size was reduced from the range of $85-280 \mu \mathrm{m}$ in Conroe oil to the range of $25-165 \mu \mathrm{m}$ in Troika oil. This is due to a lower film drainage rate and thus lower coalescence efficiency (Alvarez et al., 1994; Maggioris et al., 2000; Stamatoudis and Tavlarides, 2007). In addition, for a given wall shear stress, a lower interfacial tension between two liquid phases leads to the formation of smaller droplets because the force retaining the drop has a lower intensity (Hayworth and Treybal, 1950; Lepercq-Bost et al., 2008). It can be seen from Table 4.2-1 that the interfacial tension between two phases (oil and water) was less for Troika oil (11 mN/m) compared to the that for Conroe oil $(20 \mathrm{mN} / \mathrm{m})$, which resulted in smaller drop sizes when using Troika oil. Overall, the shifts in cumulative size distribution and the logarithm of number density of droplets towards the smaller droplet sizes with the change in oil type from Conroe oil to Troika oil in Figures 5.4-5 and 5.4-6, can be explained as follows: firstly, in turbulent viscous regime, shear forces were able to increase breakage rate; secondly, the more viscous continuous phase lowered film drainage rate and thus resulted in reduction in coalescence rate; and thirdly, lower surface tension of Troika oil decreased retaining force of droplets. All these factors could lead to a narrower droplet size distribution and also a shift in the size distribution towards the smaller droplet sizes.

In this study, the grid was partitioned into 24 parts and each simulation was run with 24 CPUs in parallel. In addition, one of the important point in this work is the impact of the mixing tank geometry on the population of dispersed phase which is described in terms of calculation of the average turbulent kinetic energy and also the contours of the volume fraction of the second phase 
in the impeller region and the circulating region for different speeds and continuous phase viscosities. Figures 5.4-7 and 5.4-3a demonstrate the contours of turbulent kinetic energy computed using the validated CFD model for two different types of oil (Troika oil and Conroe oil) at the same impeller speed.

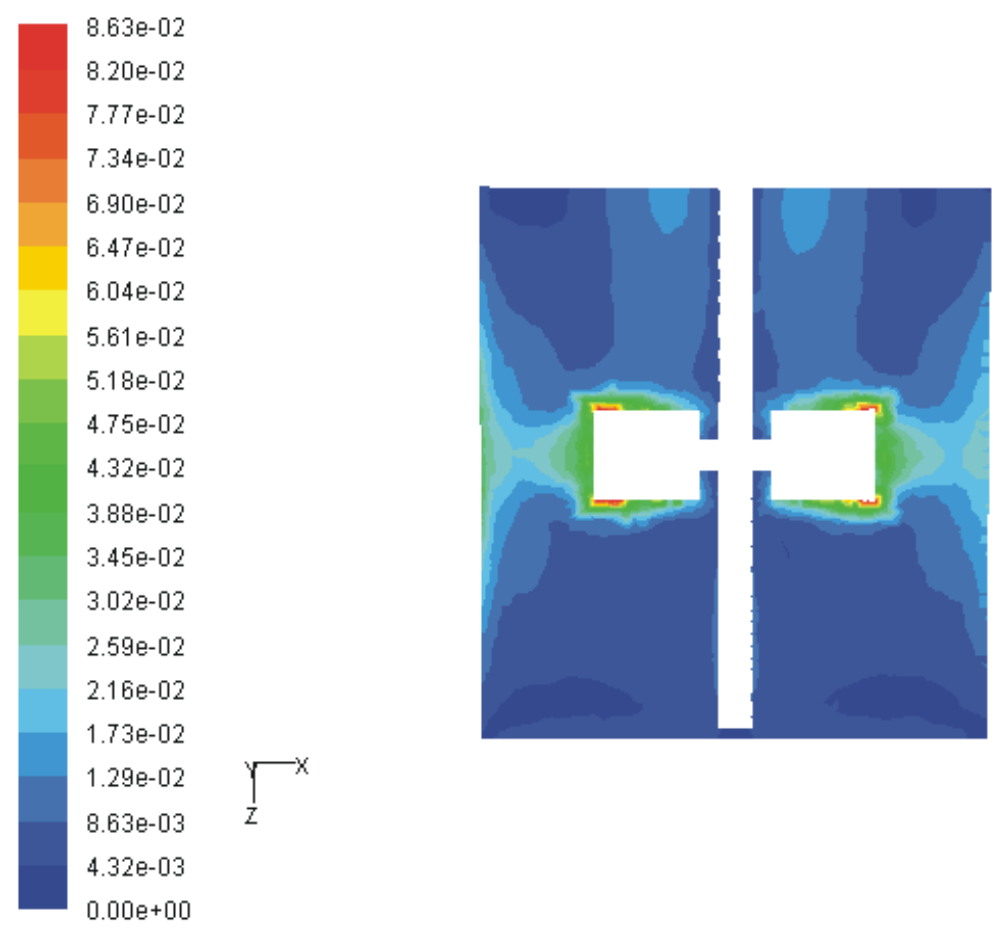

Figure 5.4-7 Contour of turbulent kinetic energy $\left(\mathrm{m}^{2} / \mathrm{s}^{2}\right)$ at $300 \mathrm{rpm}$ and the continuous phase of Troika oil with 15 vol\% water.

These results show that the difference between the average turbulent kinetic energy in the impeller region and the circulating region for the more viscous continuous phase (i.e. Troika oil) was $0.01733 \mathrm{~m}^{2} \mathrm{~s}^{-2}$, which was higher than that calculated for Conroe oil $\left(0.012 \mathrm{~m}^{2} \mathrm{~s}^{-2}\right)$. This supports the results found by Maggioris et al. (2000) regarding the effect of the continuous phase viscosity on the average turbulent kinetic energy in the impeller region and the circulating region. Figures 5.4-8 and 5.4-4a show the contour of dispersed-phase volume fraction calculated 
using the CFD model at $300 \mathrm{rpm}$ for two different types of the continuous phase (Troika oil and Conroe oil, respectively). These results revealed that the dispersed phase was more evenly distributed within the vessel when the continuous phase was Troika oil. This was due to the smaller droplet sizes and narrower size distribution achieved for the emulsion with a more viscous continuous phase.
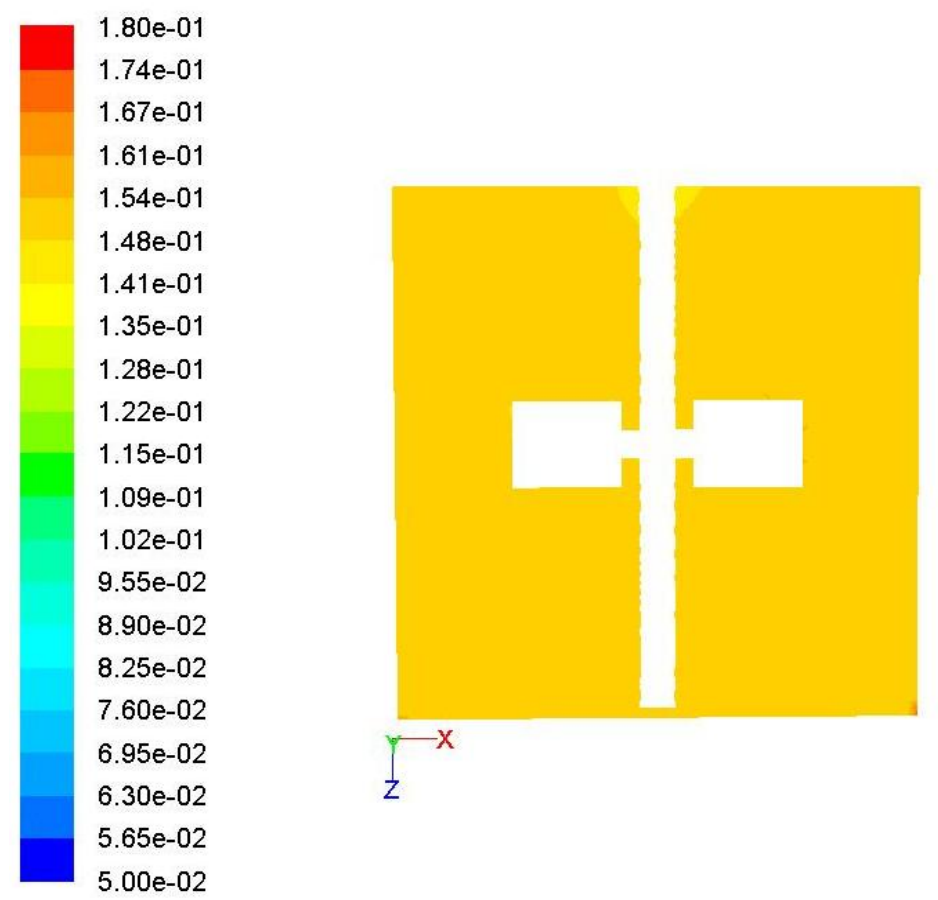

Figure 5.4-8 Contour of water volume fraction at $300 \mathrm{rpm}$ and the continuous phase of Troika oil with $15 \mathrm{vol} \%$ water.

The effect of the volume fraction of the dispersed phase (water) on the droplet size distribution was also explored using the validated CFD model developed in this study. Figures 5.4-9 and 5.4-10 depict the cumulative probability size distribution and the logarithm of number density as a function of the dispersed phase drop size computed for Conroe oil at $300 \mathrm{rpm}$ at $15 \mathrm{vol} \%$ and 25 vol\% of water, respectively. No notable difference in the CFD results was observed at 15 vol $\%$ and 25 vol $\%$ water. These CFD results are in good agreement with the experimental data 
reported in the literature (Boxall et al., 2010; Ruiz et al., 2002; Vankova et al., 2007) in which the effect of volume fraction of dispersed phase was observed to have insignificant effect on the droplet size distribution of the dispersed phase.

Liquid-liquid and gas-liquid dispersion systems play a significant role in chemical, petroleum, mining, food, and pharmaceutical industries. Prediction of drops size distribution is very crucial in designing and scaling up of these systems. The heat and mass transfer rates through interfacial areas can be determined if the size distribution is known (Luo and Svendsen, 1996).

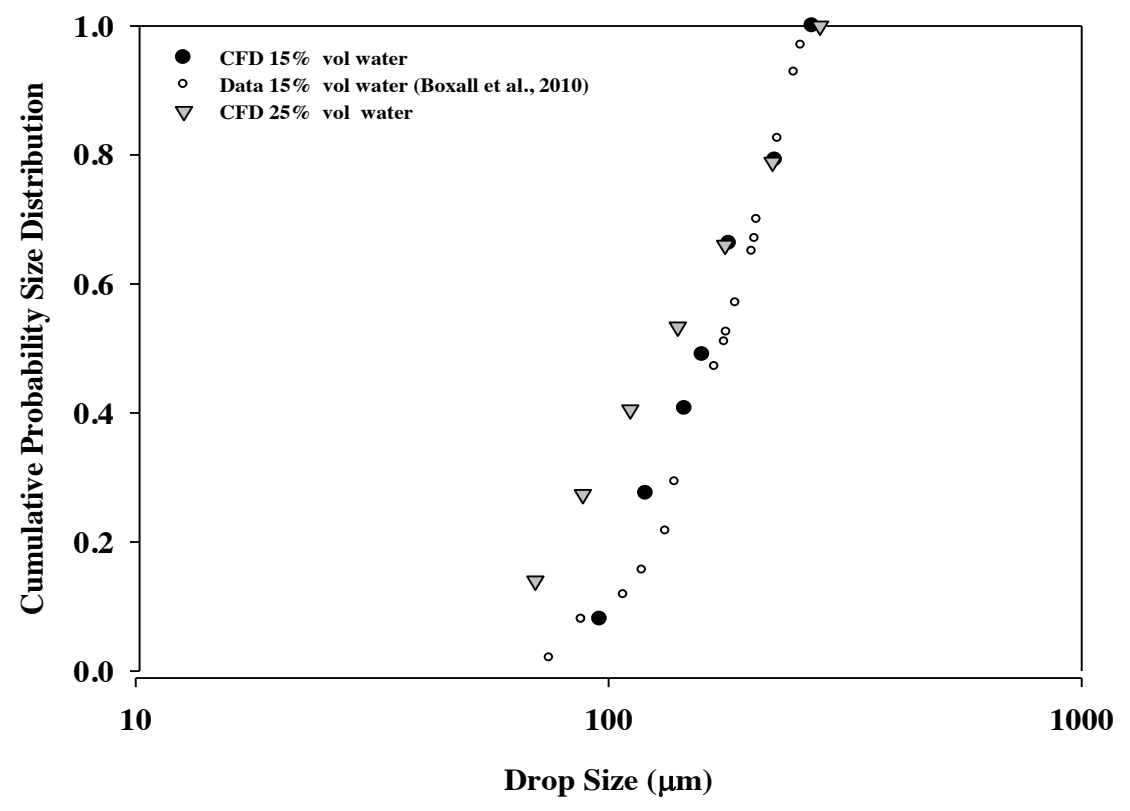

Figure 5.4-9 Cumulative probability drop size distribution versus droplet diameter for: $\bullet 15$ vol $\%$ and $\nabla 25$ vol $\%$ water at $300 \mathrm{rpm}$ with the continuous phase of Conroe oil at $20^{\circ} \mathrm{C}$. 


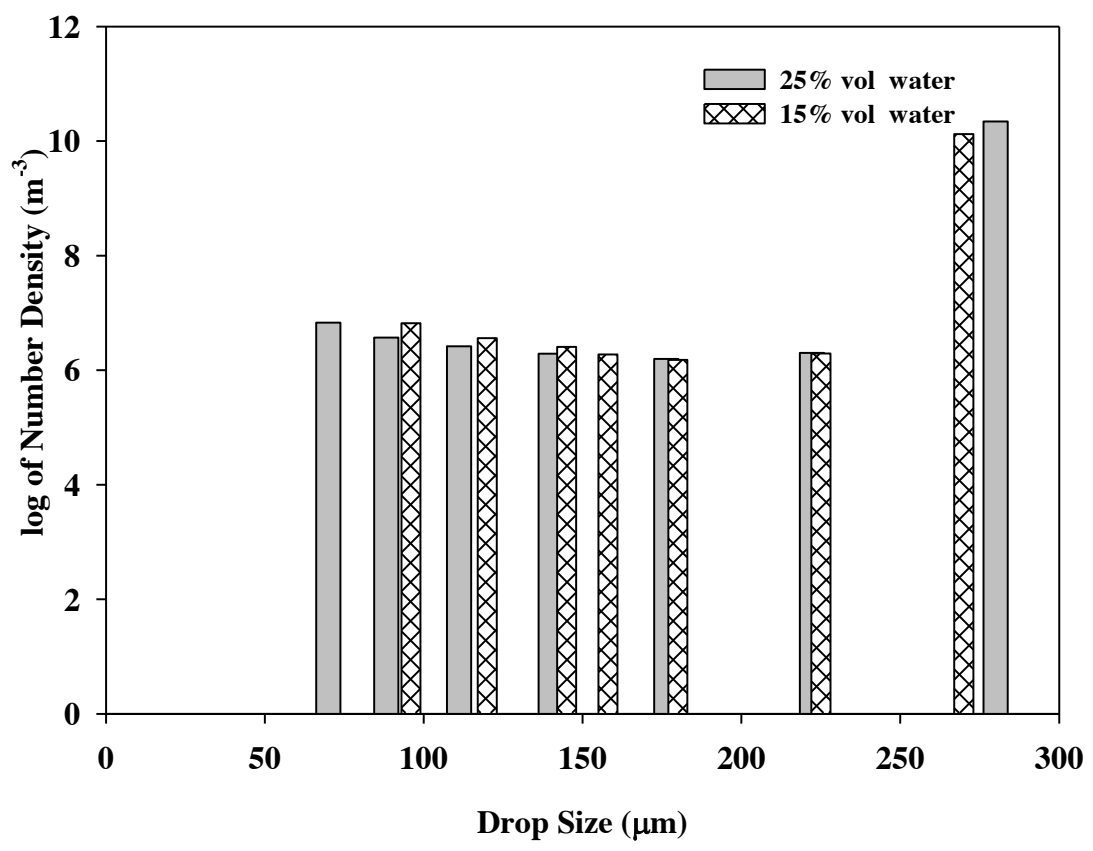

Figure 5.4-10 Logarithm of number density at 15 vol\% and 25 vol\% water at 300 rpm with the continuous phase of Conroe oil at $20^{\circ} \mathrm{C}$.

The design criteria for these systems are mostly empirical and scale up involves costly experimental programs. A major drawback of the empirical analysis is the inability to describe dispersion properties such as the droplet size distributions, interfacial surface area, and the droplet mixing rates. The CFD model developed in this study enables us to assess the effect of the design parameters and operating conditions on the performance of mixing systems utilized for the preparation of emulsions, where the prediction of dispersed phase homogeneity in tank as well as the droplet size distribution are very important for determining the interfacial areas and the heat and mass transfer rates. 


\subsubsection{Concluding remarks}

In this research, modeling of the droplet size distribution for water-in-oil emulsions was studied. Discrete model was implemented in population balance equation to investigate the droplet size distribution of the dispersed phase, i.e. water in two types of oil with different viscosities as the continuous phase. 7 bin fractions (classes) were considered for CFD simulation. The impeller rotation was incorporated into a batch small tank model under turbulent regime. Computational fluid dynamics (CFD) technique was implemented to study the effect of mixing in water-in-oil emulsion in a lab-scale tank. Breakage and coalescence phenomena were considered for size distribution using Luo model in population balance model. Logarithm of number density and cumulative probability size distribution were plotted versus droplet size of the dispersed phase at different impeller speeds, continuous phase type, and also volume fractions of water as the dispersed phase. In this work, simultaneous technique was employed to obtain an accurate solution which was in good agreement with the experimental results.

In the case of Conroe oil as the continuous phase, increase in the stirring speed shifted the drop size distribution toward the smaller drop sizes. The contour of turbulence kinetic energy in the CFD result approved enhancement in turbulence for higher impeller speeds. The homogeneity of the dispersed phase volume fraction distribution in the whole vessel was improved as the stirring speed was increased. With the change in the continuous phase from Conroe oil to Troika oil, water drop sizes were reduced. The dispersed phase was more homogeneously distributed in the vessel due to better recirculation as the result of lower droplet size in Troika oil. Besides, the average turbulent kinetic energy difference between impeller region and circulating region was higher when using Troika oil than the same difference between the two regions for Conroe oil. 
Finally, the variation in volume fraction of dispersed phase in the CFD results did not reveal any significant effect on the droplet size distribution.

In summary, the important computational issues addressed in this work are simultaneous solution of transport equations combined with turbulence and population balance, use of Eulerian approach for multiphase modeling, employment of discrete method for population balance equation, and parallelization in computation. The modeling approach developed in this study appeared to be powerful and has the potential to be extended and adapted to more complicated systems such as reactive flows (e.g. emulsion polymerization). In this case, nucleation and growth phenomena would also be imparted to the population balance equations, which was the target for the next work. 


\subsection{Use of CFD in modeling MMA solution polymerization in a CSTR (Fathi Roudsari et al., 2013)}

\subsubsection{Introduction}

A thorough literature review revealed that little attention has been devoted to the investigation of polymerization reaction conducted in continuous stirring tanks using CFD technique. The purpose of this research was to explore the effects of different operating conditions on the monomer conversion through a CFD modeling approach based on a reaction source that takes into account the reaction mechanism of MMA solution polymerization in which the gel effect is also considered. This study investigates the conversion dependence on mixing, inlet solvent concentration, reaction temperature and residence time. The polymer mass fraction was obtained directly using monomer, initiator, solvent and radical concentrations calculated by the CFD modeling.

The reactor model consists of a steady-state isothermal transport model coupled with a polymerization kinetic model. The schematic diagram of the reactor is shown in Figure 4.3-1 in Chapter 4. The mathematical model and the polymerization kinetic model are described in Chapter 4 Sections 4.3-2 and 4.3-3. The values of the reaction rate constants are listed in Table 4.3-2. The geometry grid is presented in Figure 4.3-2.

CFD simulation was used to investigate the impact of the CSTR reactor operating conditions on MMA conversion in a solution polymerization. The kinetic model took into account the gel and chain transfer agents (monomer and solvent) effects, which can affect the rate of polymerization. The experimental data were collected from open literature to validate the predictions of the 
hydrodynamic kinetic model developed for the polymerization reactor (Maschio and Moutier, 1989).

\subsubsection{Results and discussion}

Figure 5.5-1 shows profiles of MMA conversion in a solution polymerization versus initial solvent and monomer volume fractions for an impeller speed of $100 \mathrm{rpm}$, at constant temperature of $65^{\circ} \mathrm{C}$, and residence times of 45,60 , and $90 \mathrm{~min}$. Experimental data are presented by the white symbols and the CFD predictions by the black symbols for residence time of 45, 60 and 90 min, respectively. The trends of the CFD simulations are shown as smooth lines. CFD predictions agreed pretty well with conversion data all along variations of initial conditions.

Influence of solvent fraction on the reactor performance is very important since the mobility of polymer chains is greatly enhanced in the presence of solvent (Beuermann et al., 1995). As the solvent dilutes the monomer, it inherently affects the kinetics of the polymerization, especially the termination reaction (Beuermann et al., 1995). Consequently, termination of the growing polymer chains does not occur as frequently as propagation, and results in a net increase in the observed rate of polymerization. In other words, variation of $K_{t}$ with conversion is significantly affected by the starting amount of solvent, in other words, decreasing the solvent concentrations makes the gel effect more significant (Adebekun et al., 1989; Schmidt and Ray, 1981; Maschio and Moutier, 1989; Beuermann et al., 1995). In the CFD simulations, the gel effect expressed in Equation 4.3-10 caused the termination rate to decrease very rapidly. Besides, according to Equation 4.3-5, a high initial monomer concentration contributed to the generation of more live polymer radicals, which subsequently improved the polymerization rate. 


\section{Solvent volume fraction at inlet of CSTR}

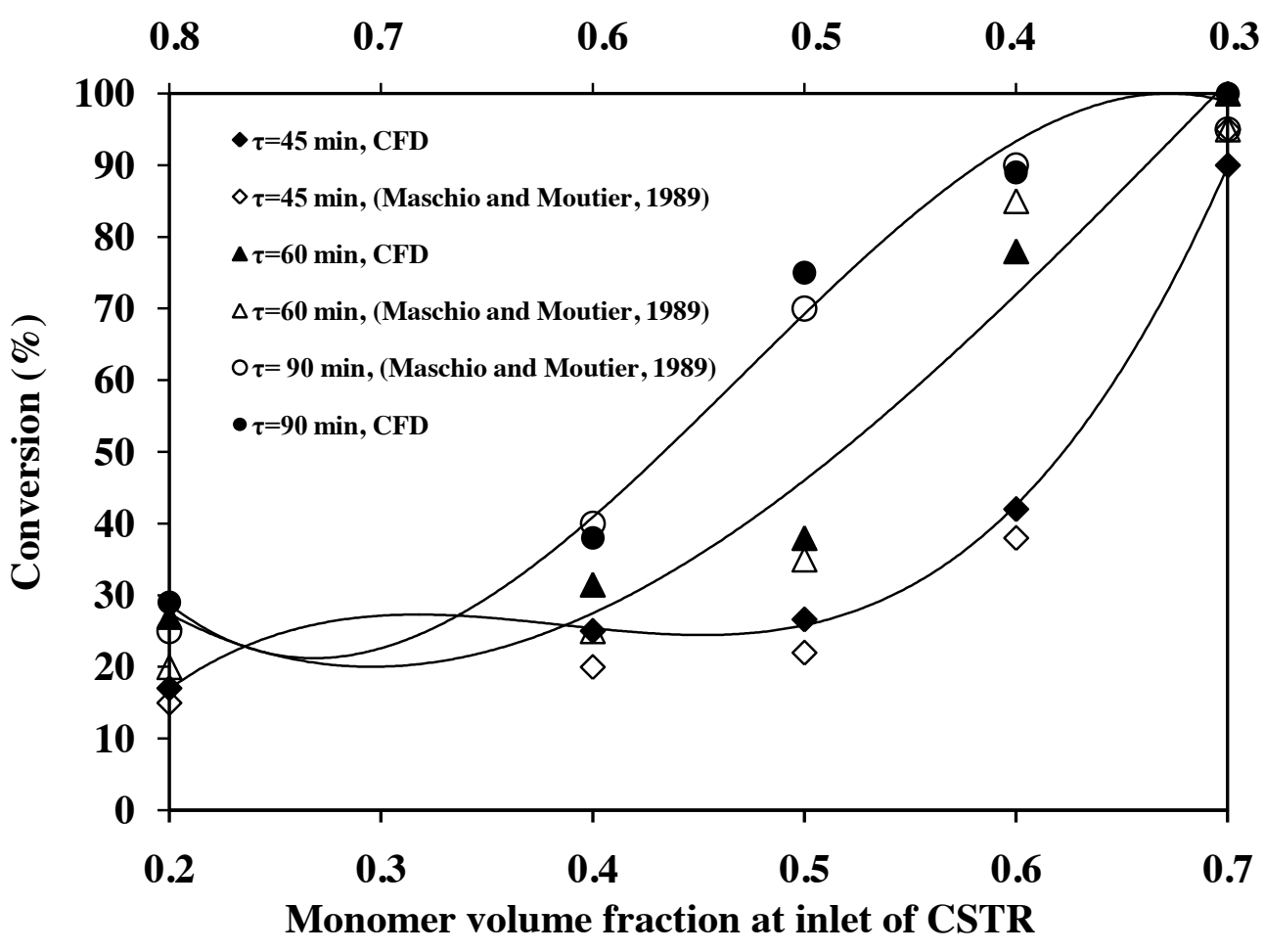

Figure 5.5-1 Effect of inlet solvent concentration on the conversion at the outlet at residence times of $\tau=45 \mathrm{~min}, \tau=60 \mathrm{~min}$, and $\tau=90 \mathrm{~min}$; impeller speed of $100 \mathrm{rpm}$ and $\mathrm{T}=65^{\circ} \mathrm{C}$.

Polymerization rate in continuous processes depends on the time the reactants spend in the reactor (Rudin and Choi, 2012). In a recent study on low solvent MMA polymerization system in a copper tube, the higher residence time allowed the flow profile to be adequately developed before the polymer product exited the reactor (Versteeg and Malalasekera, 2007). Alternatively, in high monomer concentration polymerizations, a slight increment in conversion causes a very sharp reduction in polymer chain mobility, and, inevitably, affects the termination rate (Adebekun et al., 1989; Maschio and Moutier, 1989). Also, in CFD modeling of polymerization system in a plug flow reactor (Waggoner et al., 1993), a rotating disc reactor (Lingnau and 
Meyerhoff, 1984), and a CSTR reactor (Kolhapure and Fox, 1999; Maggioris et al., 2000) higher conversion were reported at increased residence time due to proper development of polymer product at exit of reactor and also less monomer dilution in the reactor due to higher viscosity of system. These observations agreed with the results obtained by this CFD study (see Figure 5.51).

Figure 5.5-2 demonstrates the effect of temperature on conversion for the inlet monomer volume fraction of 0.7 , constant speed of $100 \mathrm{rpm}$, and residence time of $30 \mathrm{~min}$ and $45 \mathrm{~min}$. The plots exhibit the CFD approach capability to predict MMA monomer conversion at different operating conditions. Increasing the residence time from $30 \mathrm{~min}$ to $45 \mathrm{~min}$ has boosted the conversion from $15 \%$ to about $80 \%$ at $60^{\circ} \mathrm{C}$. The CFD simulation takes into account the temperature impact on the free volume fractions, kinetic constants, gel effect, medium viscosity, and density. The predictions are in accordance with data reported in the literature (Maschio and Moutier, 1989). The sharp conversion increases at $60^{\circ} \mathrm{C}$ for residence time of $40 \mathrm{~min}$ is attributed to the gel effect phenomenon, that is directly related to the reactor temperature and high inlet solvent content (Adebekun et al., 1989; Fleury et al., 1992). In CFD simulations, with reducing the operating temperature, smoother curves obtained in conversion versus time plots. Conversely, at temperatures higher than $60^{\circ} \mathrm{C}$ the curves became evidently non-linear. In this study, viscosity was function of temperature, monomer conversion, and average molecular weight and was first calculated using Equations A.2-1 and A.2-2 of appendix A.2. Also, density was considered to be a function of the temperature as shown in Equation A.2-3 of appendix A.2. Figure 5.5-3 (a to e) shows CFD contours of un-reacted MMA monomer mass fraction at constant reaction temperature of $65^{\circ} \mathrm{C}$ and different inlet volumetric monomer content of a) 0.2, b) 0.4 , c) 0.5 , d) 0.6 , and e) 0.7 , respectively. 


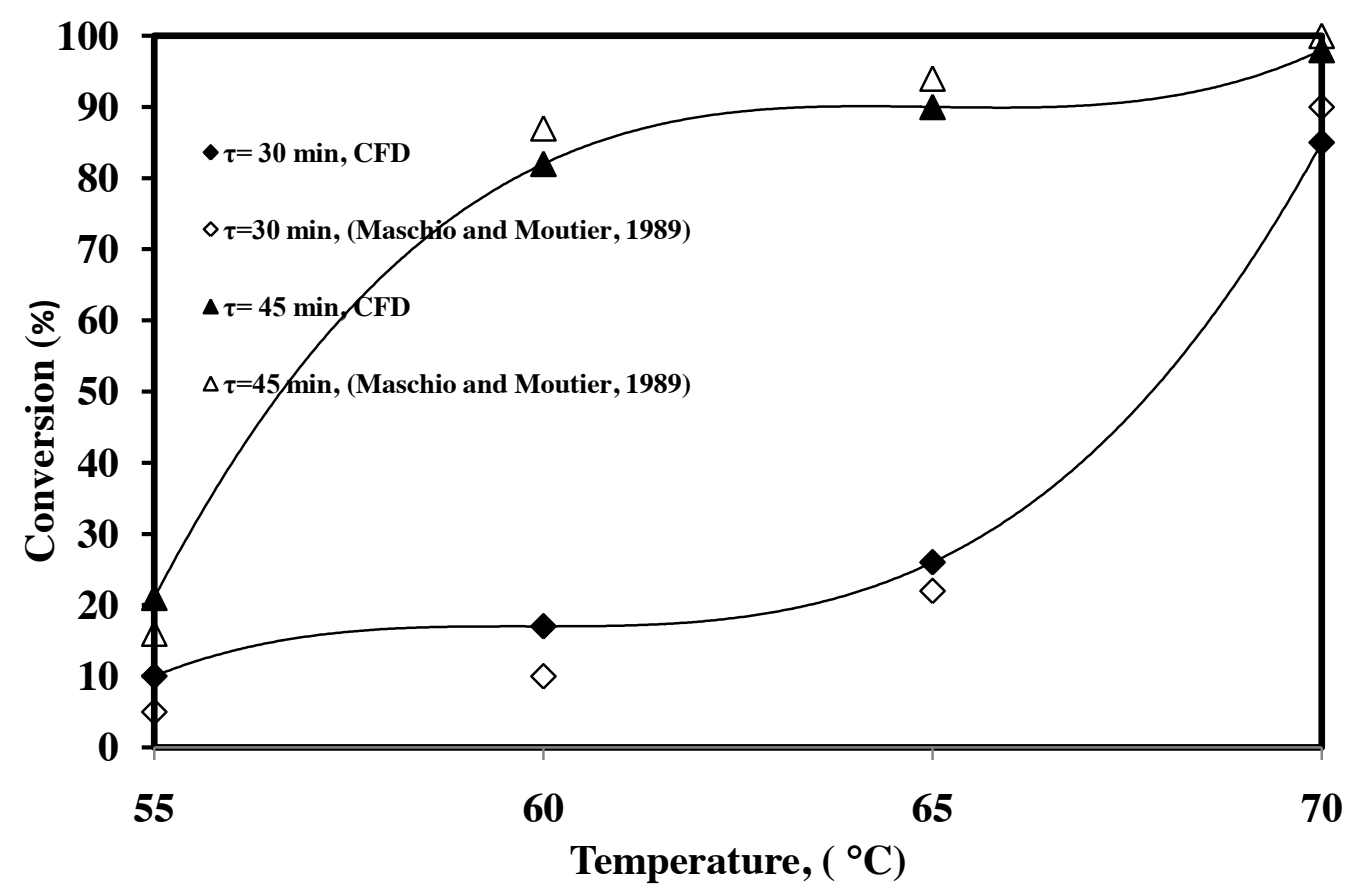

Figure 5.5-2 Effect of temperature on the conversion at $\tau=30 \mathrm{~min}$ and $\tau=45 \mathrm{~min}$ at inlet $\phi_{m}=$ 0.7 and impeller speed of $100 \mathrm{rpm}$.

As it is clearly observed, as the inlet monomer concentration was raised as shown in the colormap of each contour in Figure 5.5-3 (a to e), the un-reacted monomer fraction decreased and therefore the conversion, which is the ratio of consumed monomer to initial monomer concentration, increased. As mentioned before, this increment in conversion was due to better development of flow profile of reaction mixture and therefore higher probability of reaction between reactants.

Having validated the CFD predictions for reactor temperature 55, 60, 65, and $70^{\circ} \mathrm{C}$ (Figure 5.52), the CFD contours of un-reacted MMA monomer mass fraction are shown Figure 5.5-4 for the same range of temperatures, one residence time of $45 \mathrm{~min}$ and an initial monomer volume of $\phi_{m}$ $=0.7$. As clearly shown by the CFD results, the monomer was consumed very rapidly for the first $5^{\circ} \mathrm{C}$ when switching the reactor temperature from 55 to $60^{\circ} \mathrm{C}$, which corresponds to un- 
reacted monomer mass factions of 0.56 and 0.175 , respectively. This is a very sharp change about $38.5 \%$ monomer content loss. The change from $60^{\circ} \mathrm{C}$ to $65^{\circ} \mathrm{C}$ was $10.5 \%$ and then to $70^{\circ} \mathrm{C}$ was $7 \%$. At this point mass fraction of un-reacted monomer contour showed zero value at the lower regions of the reactor, which means a complete monomer conversion to polymer. Thus, the CFD simulation confirms the significant influence of the reactor temperature on conversion. Several simulation runs were conducted to examine the influence of agitation on the reactor performance. Figure 5.5-5 shows plots of MMA conversion at the reactor outlet versus impeller speed for different monomer inlet volume fractions and residence times. The temperature was kept constant at $65^{\circ} \mathrm{C}$. At the residence time of $45 \mathrm{~min}$, as the impeller speed was increased from $50 \mathrm{rpm}$ to $100,200,300$, and $500 \mathrm{rpm}$, respectively, and for each inlet monomer volume fraction, the conversion gradually decreased. The maximum conversion drop between lowest stirring speed (i.e. $50 \mathrm{rpm}$ ) and the highest impeller speed (i.e. $500 \mathrm{rpm}$ ) was $2 \%$ for $\varphi_{m}=0.2$ and 0.4 ; $2.5 \%$ for $\varphi_{m}=0.6$ and $5 \%$ reduction for $\varphi_{m}=0.7$. In case of residence time of 60 minutes, the conversion between impeller speeds of 50 and $500 \mathrm{rpm}$ descended about $2 \%$ for $\varphi_{m}=0.2$ and $\varphi_{m}$ $=0.4 ; 5 \%$ for $\varphi_{m}=0.6$ and zero for $\varphi_{m}=0.7$. The results indicate that for high residence time $(\tau=$ $60 \mathrm{~min})$ and high initial monomer volume fraction $\left(\varphi_{m}=0.7\right)$, the conversion is almost independent of stirring speed. The conversion decline at higher impeller speed may be due to short-circuiting of un-reacted monomer and also inlet/outlet location. A similar decrease in conversion at higher stirring speeds was reported for styrene bulk polymerization in a CSTR (Kolhapure and Fox,, 1999; Maggioris et al., 2000). However, Erdogan et al. (2002) experimentally showed an increase in conversion with stirring rate to a certain point, but further increase in impeller speed reversed the conversion profile. The improvement in conversion was attributed to a better initiator efficiency as the mixing was enhanced. 


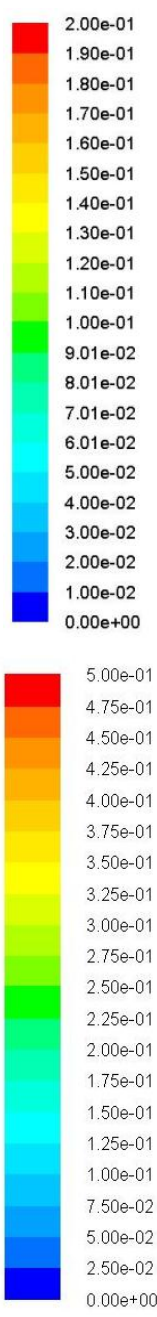

a)

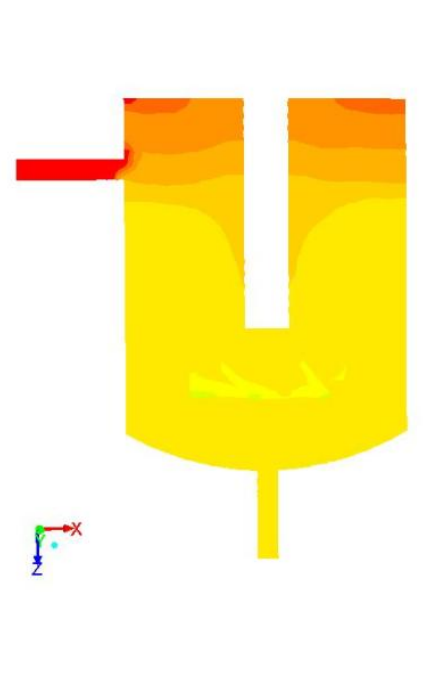

c)

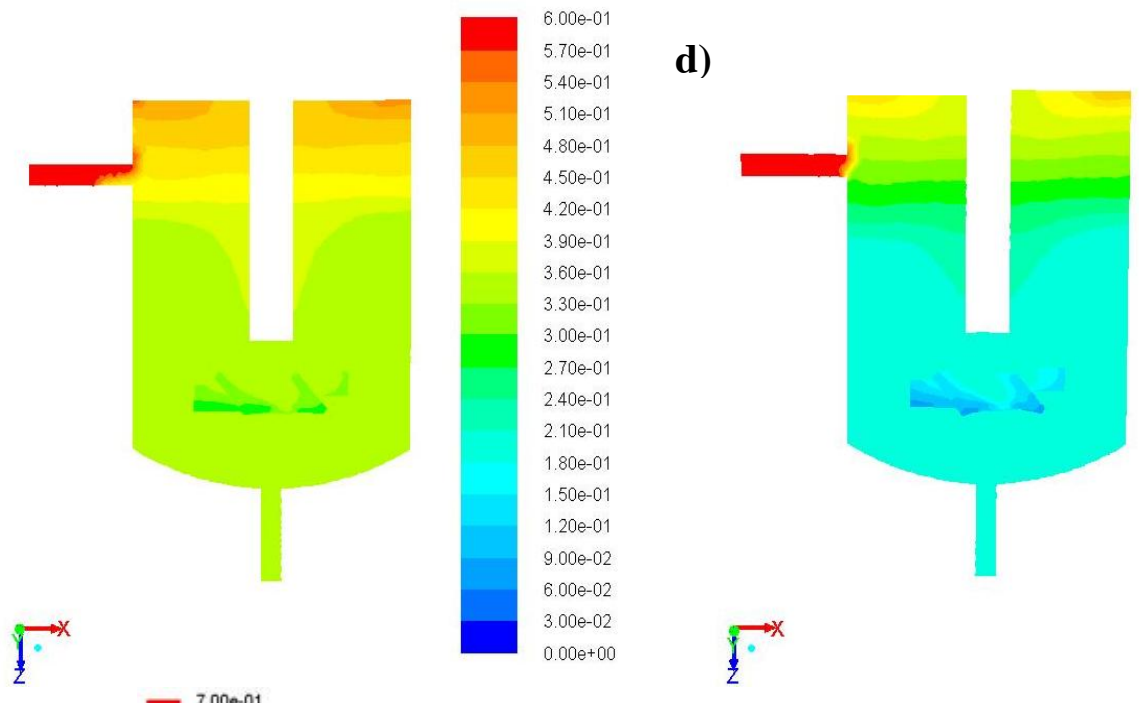

b)

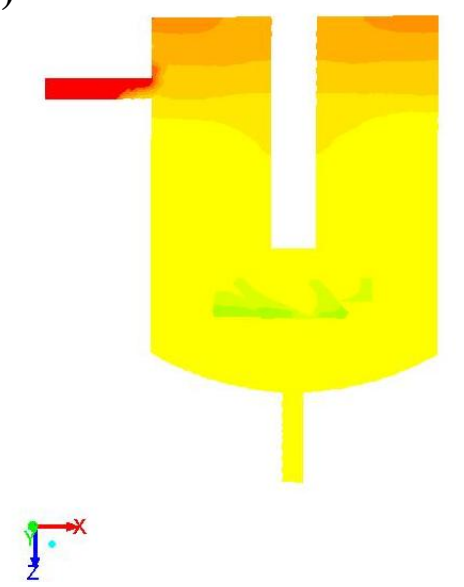

d)

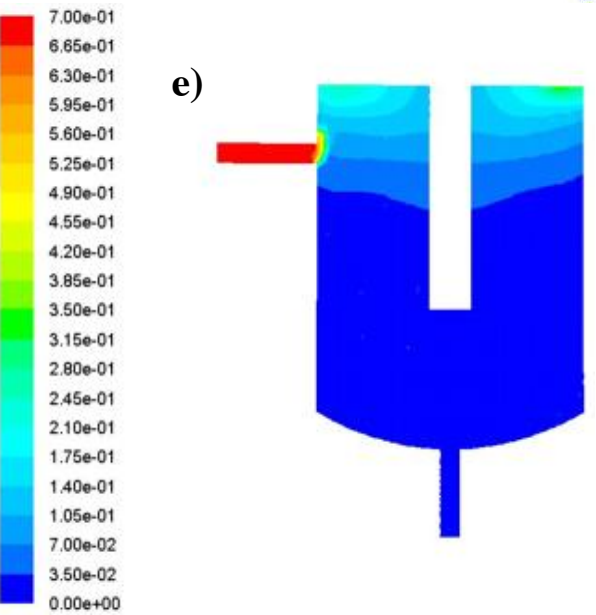

Figure 5.5-3 Contours of MMA monomer mass fraction at inlet monomer concentraton of: a) $\phi_{m}=0.2$, b) $\phi_{m}=0.4$, c) $\phi_{m}=0.5$, d) $\phi_{m}=0.6$, and e) $\phi_{m}=0.7$ at residence time of $\tau=60 \mathrm{~min}$, impeller speed of $100 \mathrm{rpm}$, and $\mathrm{T}=65^{\circ} \mathrm{C}$. 
a)

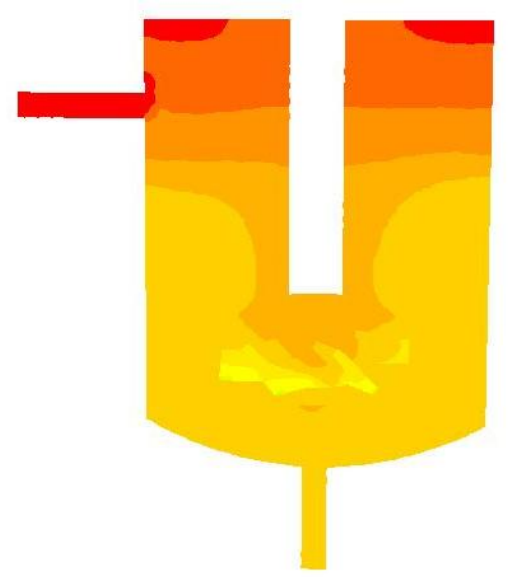

c)

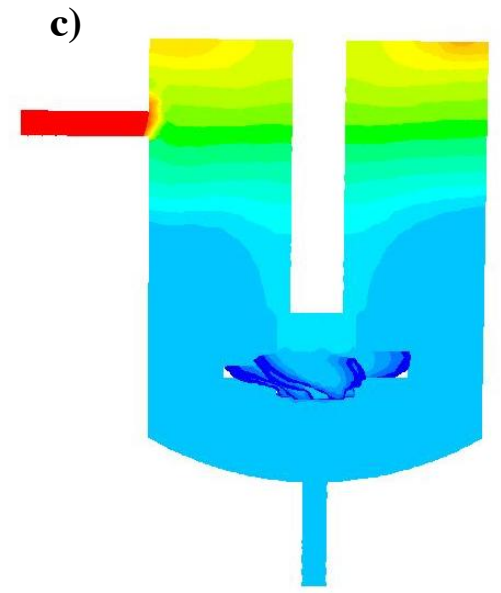

b)

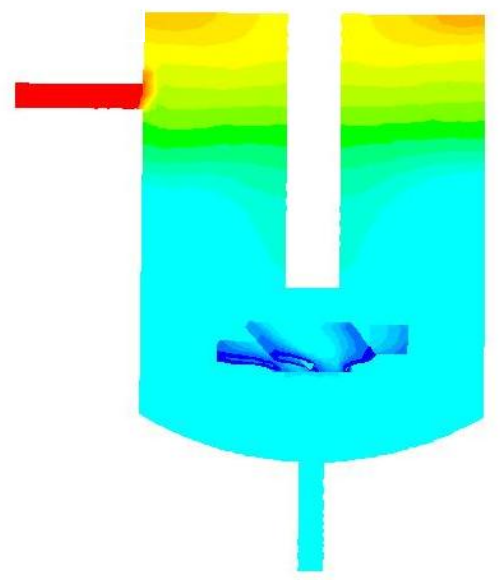

(d

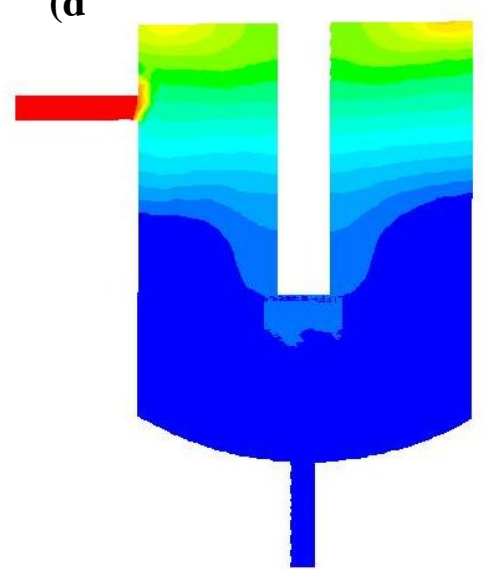

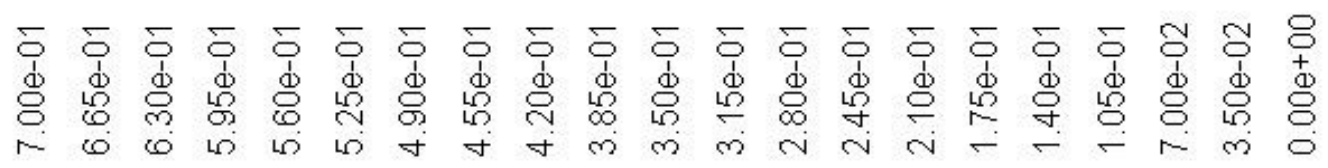

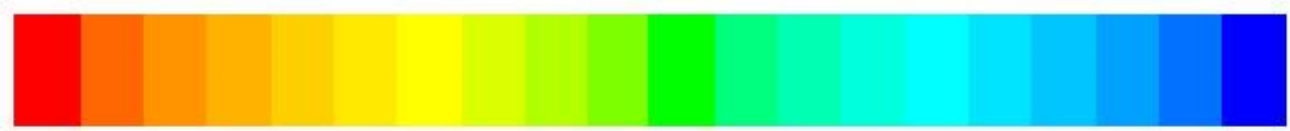

Figure 5.5-4 Contours of MMA monomer mass fraction at residence time of $\tau=45 \mathrm{~min}$, inlet monomer concentration of $\phi_{m}=0.7$, impeller speed of $100 \mathrm{rpm}$ and reaction temperatures of: a) $55^{\circ} \mathrm{C}$, b) $60^{\circ} \mathrm{C}$, c) $65^{\circ} \mathrm{C}$, and d) $70^{\circ} \mathrm{C}$. 
b)

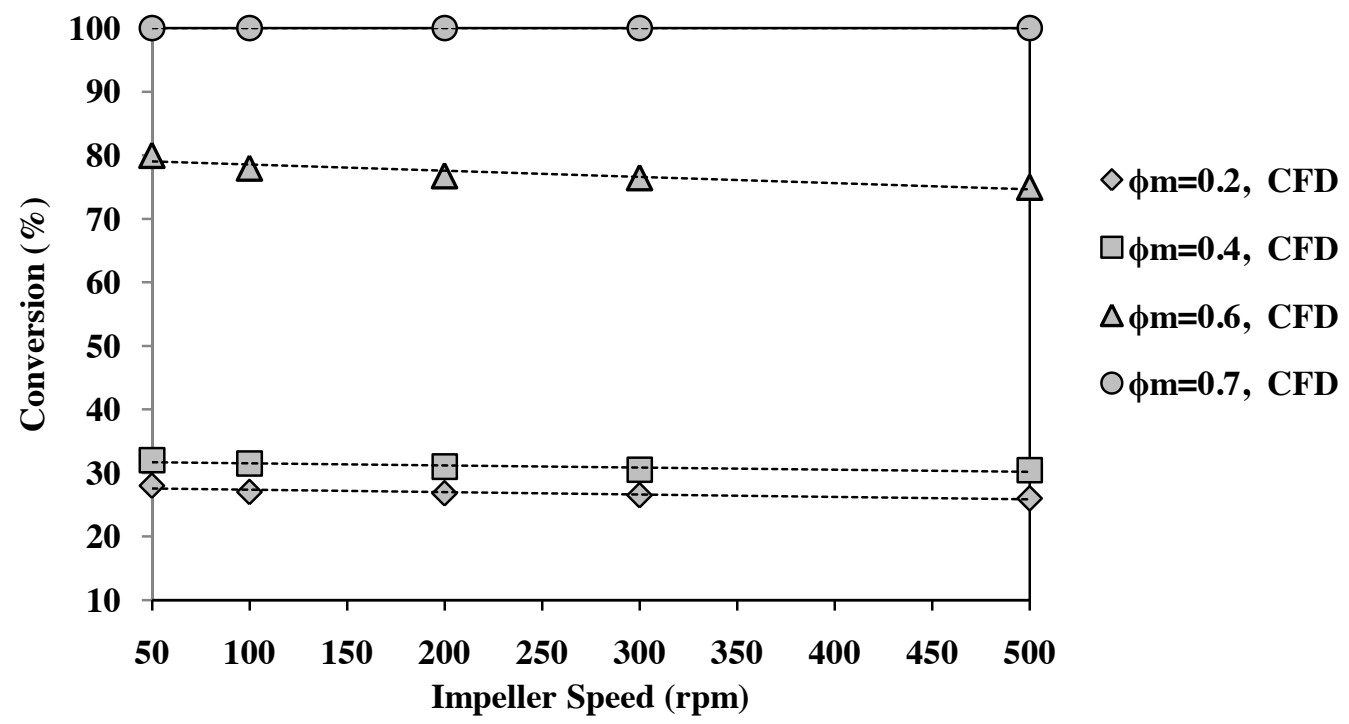

a)

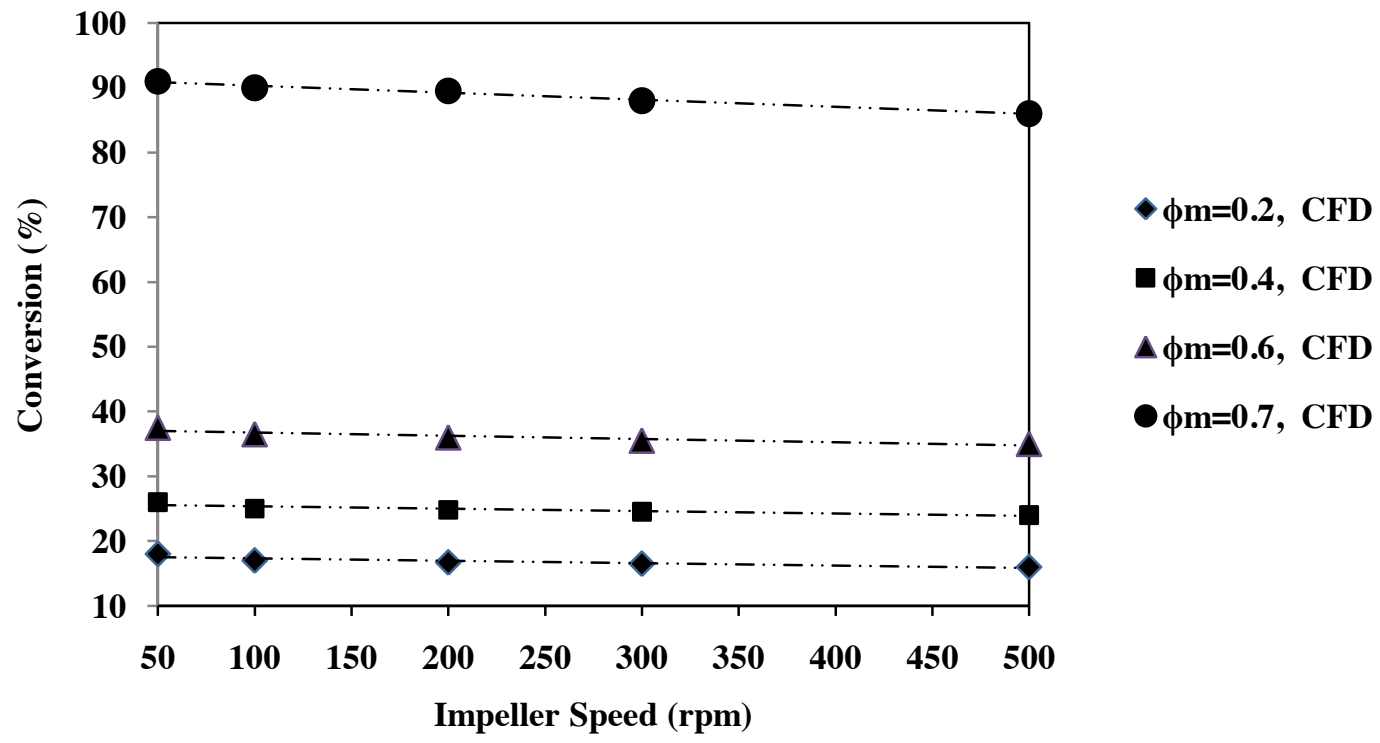

Figure 5.5-5 Effect of impeller speed on the conversion as a function of the inlet MMA monomer concentration at: a) $\tau=45 \mathrm{~min}$, and b) $\tau=60 \mathrm{~min}$ and $\mathrm{T}=65^{\circ} \mathrm{C}$. 
Figure 5.5-6 shows the contours of un-reacted MMA mass fractions at different impeller speeds for $\varphi_{m}=0.6$ and $\tau=60 \mathrm{~min}$. CFD simulations revealed that mixing was not quite good at the lower impeller speeds of 50 and $100 \mathrm{rpm}$. Thus, the upper section of the reactor remained rich in un-reacted MMA, whereas MMA mass fraction dropped to 0.15 approximately in the region around the impeller and near the base of the tank. It can be easily observed that the monomer mass fraction changeed drastically from layer to layer of the reaction mixture especially at the upper section of the reactor. As the impeller speed was brought up to 200, 300, and $500 \mathrm{rpm}$, recirculation of the mixture was improved, thus rendering more uniform distribution of monomer content.

To compare the monomer fraction contours at impeller speeds of 50 and $500 \mathrm{rpm}$, seven surfaces were generated, by means of CFD, at different locations perpendicular to the impeller shaft direction (Figure 5.5-7).

Obviously, at $50 \mathrm{rpm}$, the first four planes indicate a limited polymer production. But at an impeller speed of $500 \mathrm{rpm}$, a circulation enhancement occurred resulting in a sharp drop of monomer content, particularly in the upper reactor region. Therefore, the CFD simulation was capable of exhibiting the existence of different mixing layers as well as dead zones in the reactor when the agitation was slow. Conversely, the un-reacted monomer content did not significantly change in the reactor planes (v-vii) whereas the impeller speed was altered from 50 to $500 \mathrm{rpm}$. In other words, the polymer fraction and conversion did not show a profound change, which was $5 \%$ only. That can be attributed to the location of the outlet stream that was placed at the lower region, i.e. bottom of tank. In this case, although with increase in impeller speed, the recirculation was getting better in the upper regions of the tank, the conversion did not significantly change due to fairly good agitation in lower region of tank in both low and high 

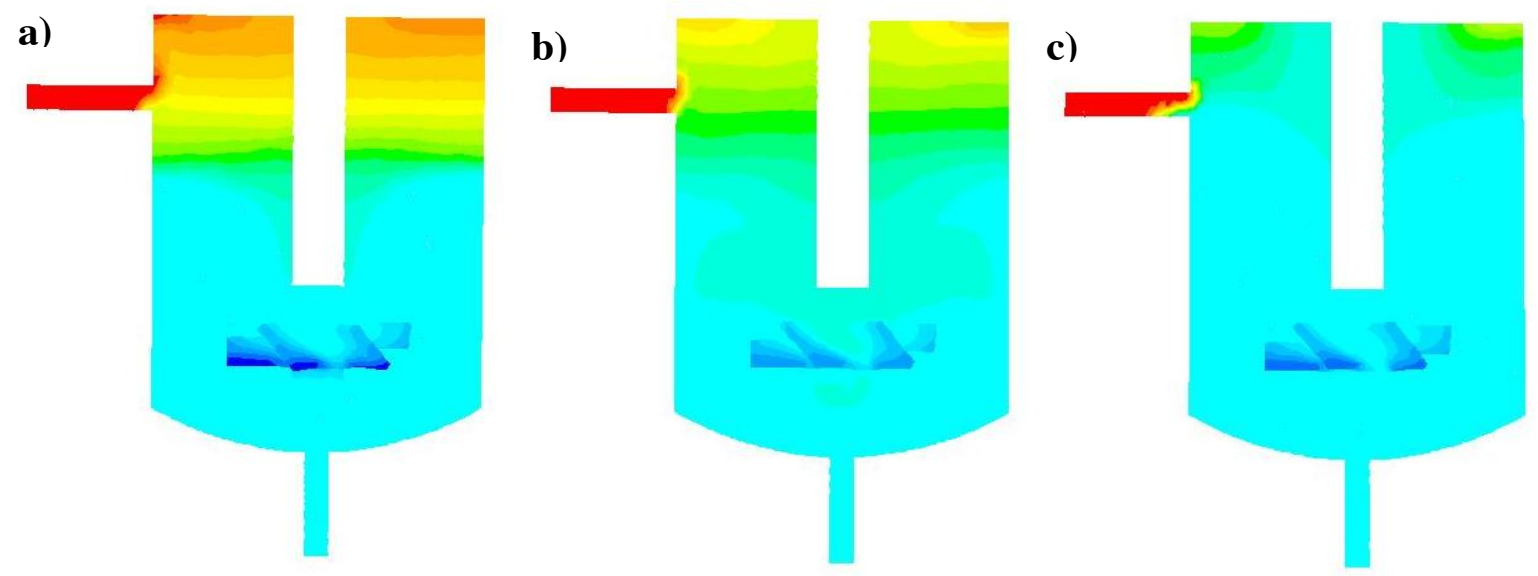

d)

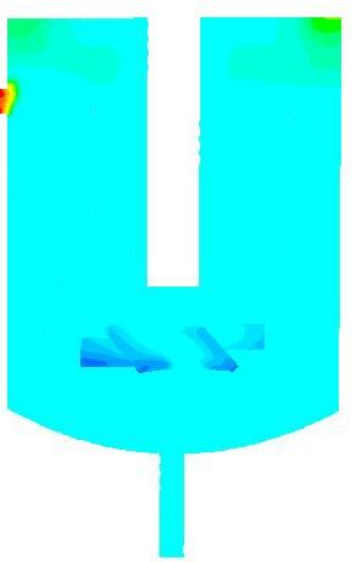

e)

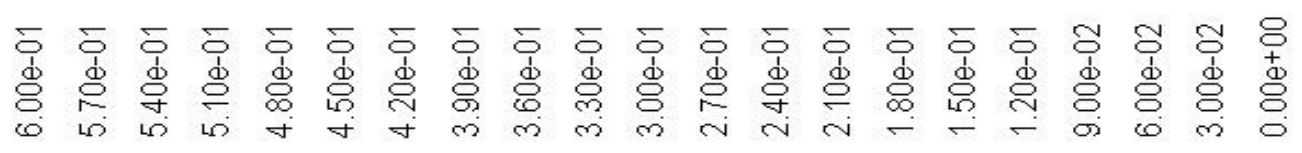

Figure 5.5-6 Contours of MMA monomer mass fraction at $\tau=60 \mathrm{~min}, \phi_{m}=0.6$ and impeller speed of: a) $50 \mathrm{rpm}$, b) $100 \mathrm{rpm}$, c) $200 \mathrm{rpm}$, d) $300 \mathrm{rpm}$, and e) $500 \mathrm{rpm}, \mathrm{T}=65^{\circ} \mathrm{C}$.

speed. It must be mentioned that the conversion in this CFD study was obtained at the outlet location of the reactor.

Influence of the residence time on homogeneity and conversion at a relatively low solvent content $\left(\varphi_{s}=0.3\right)$ is depicted by CFD distribution layers in Figures 5.5-8 and 5.5-9, keeping the 
residence time at $45 \mathrm{~min}$ and varying the impeller speed from 50 to $500 \mathrm{rpm}$. The un-reacted monomer was subjected to faster recirculation, thus resulted in a more uniform MMA content dispersion inside the reactor space. This improved homogeneity resulted in lower conversion at the outlet (about 5\% reduction in conversion). However, at $\tau=60 \mathrm{~min}$ (Figure 5.5-9) and variation of the agitation speed from $50 \mathrm{rpm}$ to $500 \mathrm{rpm}$, the monomer spent more time in the reactor and its mass fraction dropped to almost zero.

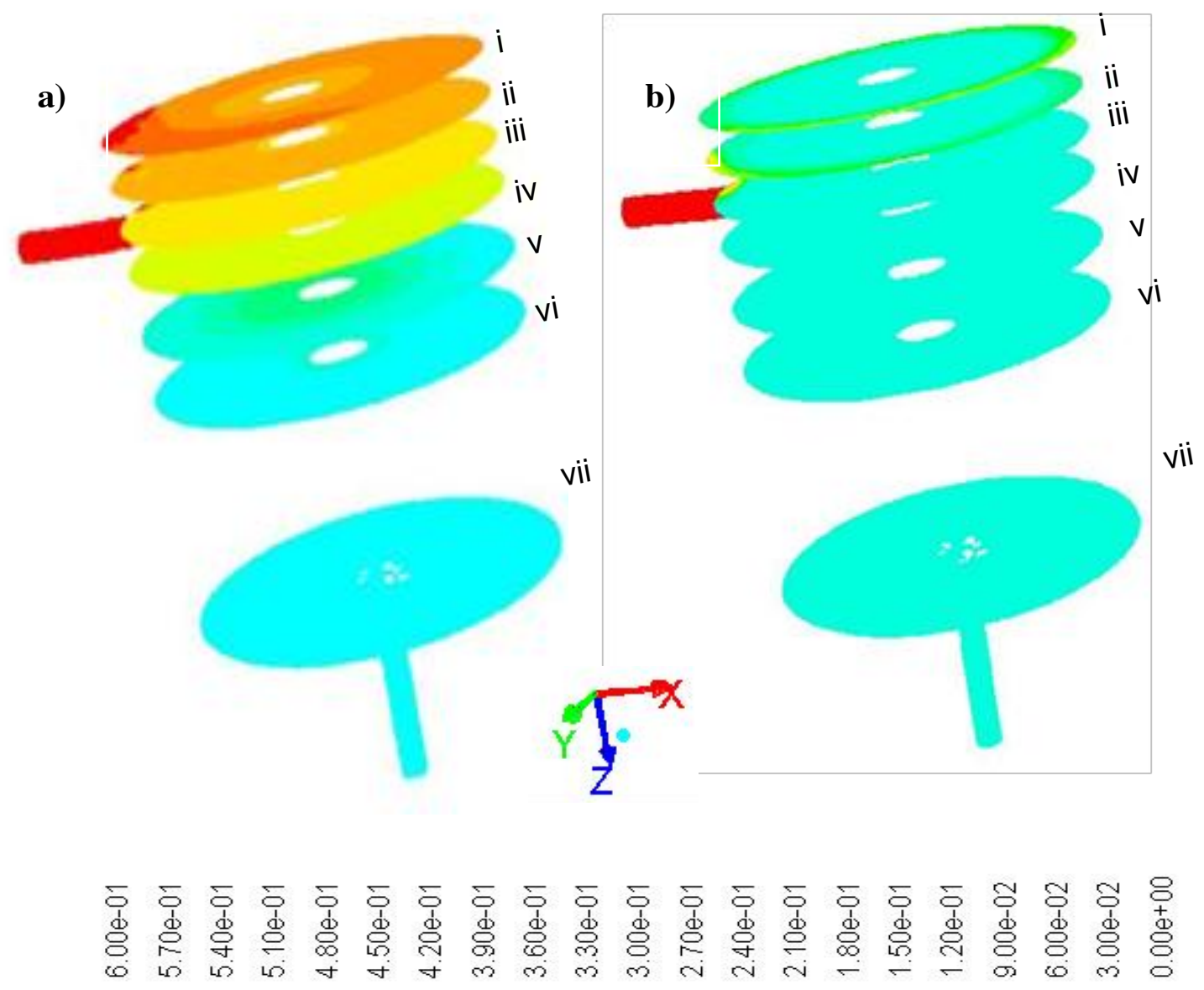

Figure 5.5-7 Contour of MMA monomer mass fraction in planes perpendicular to shaft direction at locations of i) 0 , ii) $0.01 \mathrm{~m}$, iii) $0.02 \mathrm{~m}$, iv) $0.03 \mathrm{~m}$, v) $0.045 \mathrm{~m}$, vi) $0.06 \mathrm{~m}$, vii) $0.115 \mathrm{~m}$ for $\tau$ $=60 \mathrm{~min}, \phi_{m}=0.6$ and at the impeller speeds of: a) $50 \mathrm{rpm}$, and b) $500 \mathrm{rpm}$. 
Under these operating conditions, the CFD simulation predicted the conversion of almost $100 \%$ even at a low impeller speed. It can be concluded that a full conversion is possible when the flow is fully developed providing an enhancement of macro mixing that can in turn, affect the propagation rate.
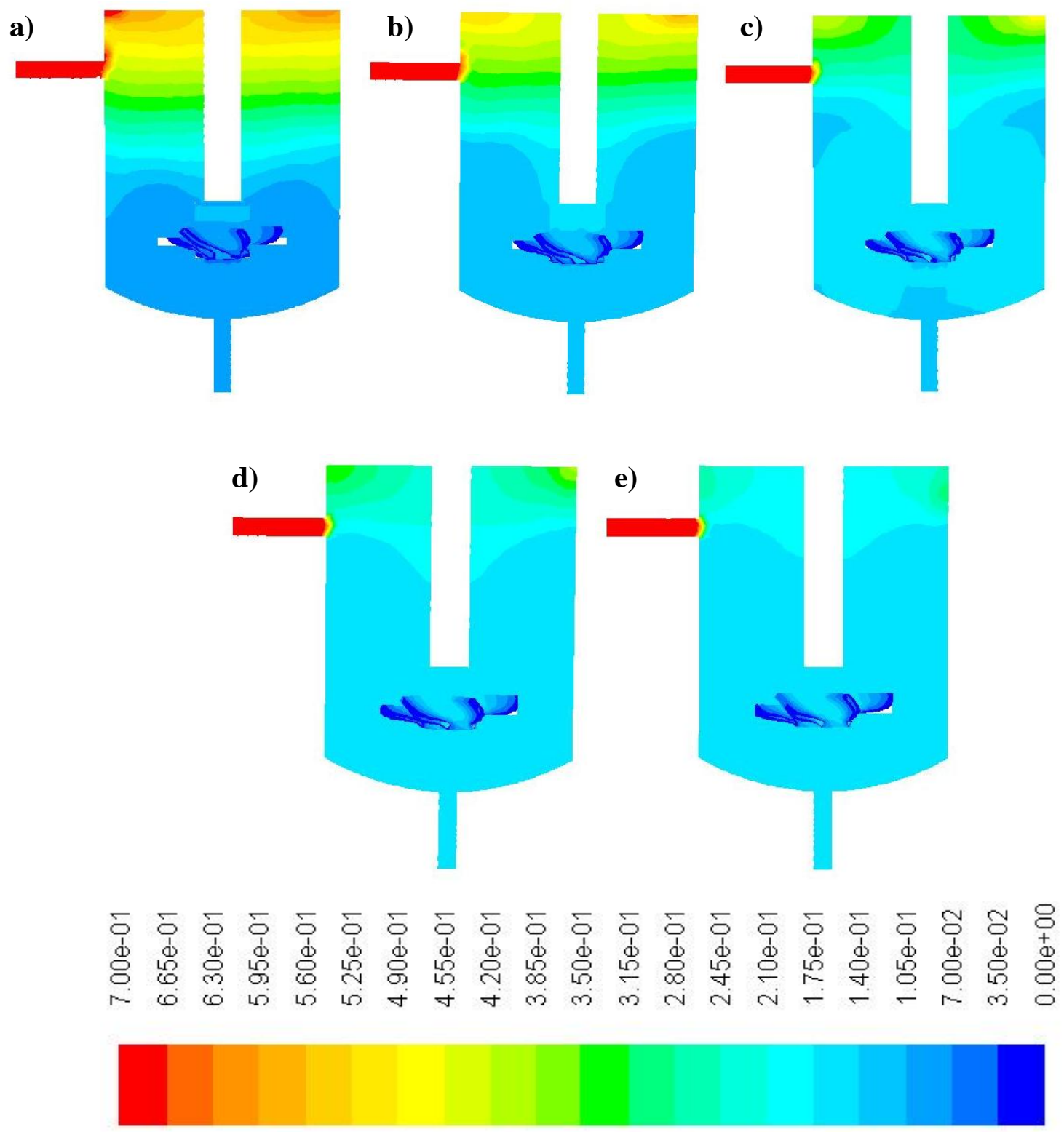

Figure 5.5-8 Contours of MMA monomer mass fraction at $\tau=45 \mathrm{~min}, \phi_{m}=0.7$, and impeller speeds of: a) $50 \mathrm{rpm}$, b) $100 \mathrm{rpm}$, c) $200 \mathrm{rpm}$, d) 300 , and e) $500 \mathrm{rpm}, \mathrm{T}=65^{\circ} \mathrm{C}$. 

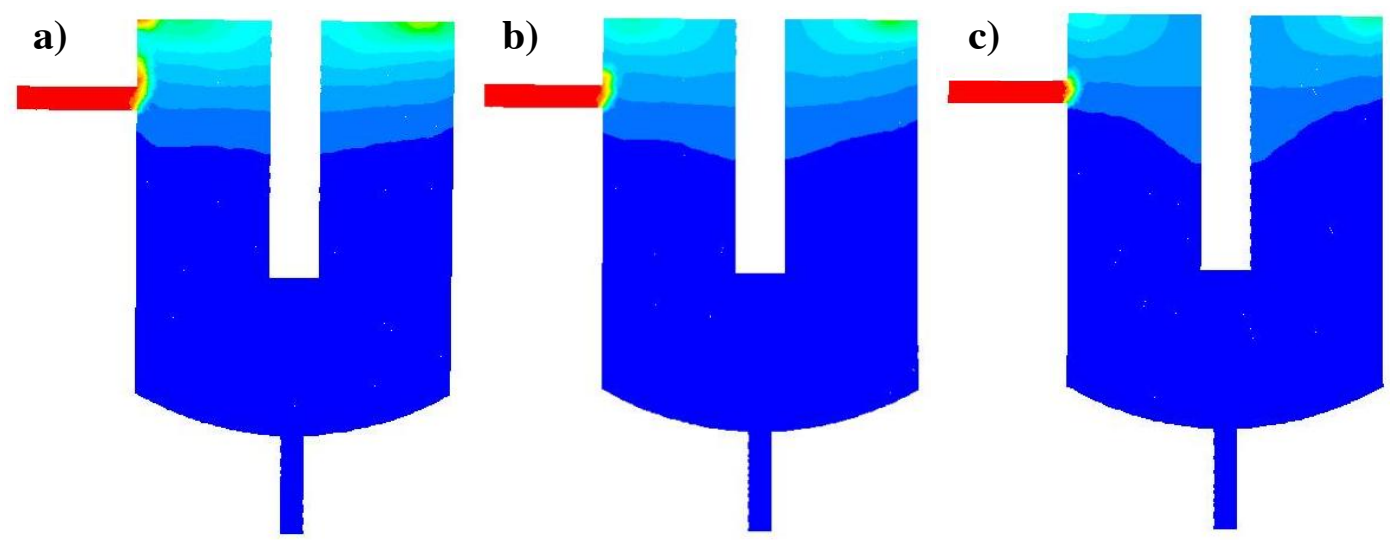

\begin{abstract}
d)
\end{abstract}

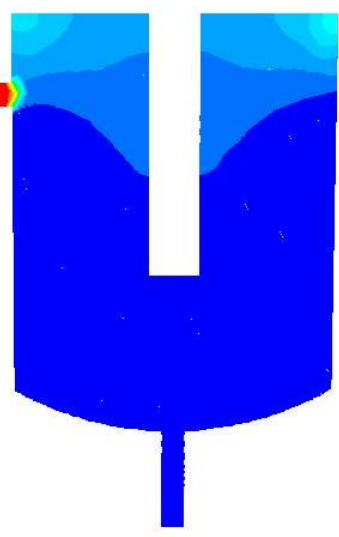

e)
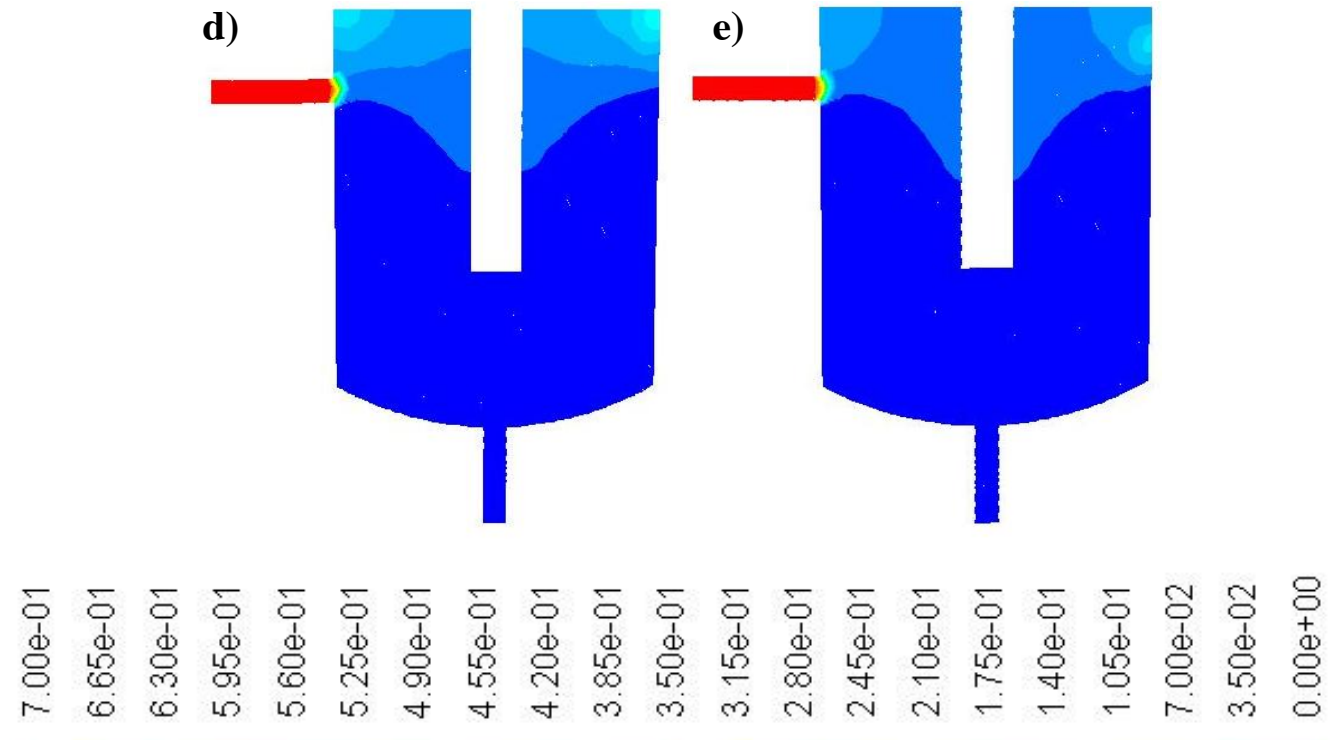

Figure 5.5-9 Contours of MMA monomer mass fraction at $\tau=60 \mathrm{~min}, \phi_{m}=0.7$, and impeller speeds of: a) $50 \mathrm{rpm}$, b) $100 \mathrm{rpm}$, c) $200 \mathrm{rpm}$, d) 300 , and e) $500 \mathrm{rpm}, \mathrm{T}=65^{\circ} \mathrm{C}$. 


\subsubsection{Concluding remarks}

Computational fluid dynamics (CFD) technique was employed to investigate the influence of operating conditions (e.g. impeller speed, initial monomer concentration, residence time, and reaction temperature) on the conversion and homogeneity state in a lab-scale CSTR utilized for MMA polymerization. The CFD model developed in this study was validated successfully with the experimental data. Each run was performed using $36 \mathrm{CPUs}$ supercomputing facilities. The CFD modeling incorporated the integration of continuity, momentum, energy and species continuity equations along with a polymerization source term. Impeller rotation was modeled in a 3D flow domain under isothermal and laminar regime. The source term was a function of thermal and chemical initiations, chain transfer to monomer and solvent as well as the gel effect. An increase of temperature or initial monomer concentration enhanced monomer conversion, which exhibited a sharper increment with higher residence time. Besides, contours of monomer mass fraction conceptually allowed us to visualize the reaction progress in the reactor. At low impeller speed and lower inlet monomer concentration, imperfect mixing was revealed at the upper region of the reactor, but a raise in the impeller speed improved the degree of homogeneity. The key computational issues investigated in this study incorporated a relatively complicated kinetics including CTA and gel effect into the source term, then solution of transport equations combined with the mentioned source term, and parallelization in computation. The CFD modeling approach adopted in this study can easily reveal the impact of the internal flow pattern and the external flow condition on the performance of the polymerization reactor. This CFD modeling can be used for the reactor design, process optimization, and the forecast of the end product quality. 


\section{Chapter 6}

\section{OVERALL CONCLUSION AND RECOMMENDATIONS FOR FUTURE WORK}

\subsection{Overall conclusion}

In this study, the experimental and computational fluid dynamics (CFD) techniques were utilized to analyze the effect of mixing parameters on the methyl methacrylate emulsion polymerization. The following main conclusions were achieved in five sections of this study.

Section 1: Mixing effect on emulsion polymerization in a batch reactor equipped with the pitched blade impeller

An increase in the impeller speed enhanced the polymer characteristics such as the monomer conversion, particle size, and molecular weight up to an optimum speed (250 rpm) and further increase in the rotational speed (from 250 to $350 \mathrm{rpm}$ ) resulted in the reduction of the mentioned polymer properties. Installation of the baffles decreased the monomer conversion and number of particles, where as the particle size and the molecular weight of the polymer produced in the reactor with baffles were slightly increased. Also, use of baffles led to a polymer product with a narrower size distribution.

\section{Section 2: Impact of impeller type on MMA emulsion polymerization in a batch reactor}

Using the Rushton impeller showed relatively higher values for conversion, average molecular weight, particle size and glass temperature compared to those obtained using the pitched-blade 
turbine at $100 \mathrm{rpm}$. However, the increase in the mentioned properties with the elevation of impeller speed was less significant than the gain obtained using the pitched-blade turbine. With the use of two pitched-blade impeller, the monomer conversion, average molecular weight and particle size at low stirring rate of $100 \mathrm{rpm}$ were higher than those achieved using a single pitched-blade impeller.

\section{Section 3: CFD Analysis of mixing in emulsion polymerization of MMA}

The CFD model was validated with experimental data. It was employed to investigate the effects of the impeller speed, impeller type, and baffles on MMA conversion, particle size, and number density distribution of polymer particles. The CFD modeling revealed the decrease in conversion with the use of baffles due to the accumulation of monomer droplets behind the baffles. The distribution of polymer volume fraction within the reactor equipped with the pitched blade impeller was more uniform than that for the Rushton impeller due to a better circulation and higher flow number.

\section{Section 4: CFD modeling of the mixing of water in oil emulsion}

In the case of Conroe oil as the continuous phase, increase in the stirring speed shifted the drop size distribution toward the smaller drop sizes. The homogeneity of the dispersed phase volume fraction distribution in the whole vessel was improved as the stirring speed was increased. With the change in the continuous phase from Conroe oil to Troika oil, smaller water drop sizes were obtained. The dispersed phase was more homogeneously distributed within the vessel due to better recirculation as the result of lower droplet size in Troika oil. 


\section{Section 5: Use of CFD in modeling MMA solution polymerization in a CSTR.}

As expected, an increase of temperature or initial monomer concentration enhanced monomer conversion, which exhibited a sharper increment with residence time. Besides, contours of monomer mass fraction conceptually allowed visualization of the reaction progress in the reactor. At low impeller speed and small inlet monomer concentration, imperfect mixing appeared at the upper region of the reactor, but a raise in the impeller speed improved the degree of homogeneity. 


\subsection{Recommendations for future work}

Based on the experimental and CFD results achieved in this study, the following topics are suggested for future work:

- The effect of mixing parameters on emulsion polymerization in a semi-batch reactor should be evaluated.

- The breakage and agglomeration should be incorporated into the emulsion polymerization CFD model along with nucleation and growth phenomena.

- The modeling of the molecular weight distribution for an emulsion polymerization should be included in the CFD model. 


\section{NOMENCLATURE}

$b \quad$ Luo model breakage parameter in Equation 4.2-14

$B \quad$ Birth rate in Equation 4.2-15, $\mathrm{m}^{-3} \mathrm{~s}^{-1}$

$C_{1 \varepsilon} \quad k-\varepsilon$ turbulent model constant in Equation 4.1-23

$C_{2 \varepsilon} \quad k-\varepsilon$ turbulent model constant in Equation 4.1-23

$C_{3 \varepsilon} \quad k-\varepsilon$ turbulent model constant in Equation 4.1-23

$C_{D} \quad$ Drag coefficient in Equation 4.1-11

$C_{\mu} \quad$ Turbulent model constant Equation 4.1-20

$c_{f} \quad$ Luo model breakage parameter in Equation 4.2-14

$C_{m 0} \quad$ Initial monomer concentration in Equation 4.1-45, $\mathrm{kg} \mathrm{m}^{-3}$

$C_{m t} \quad$ Monomer concentration at time $t$ in Equation 4.1-44, $\mathrm{kg} \mathrm{m}^{-3}$

D Diameter in Equations 4.1-10, 4.2-12 and 4.2-14, $\mathrm{m}$;

$D_{32} \quad$ Sauter mean particle size in Equation 5.3-1, $m$

$D_{\text {turb }} \quad$ Turbulent diffusivity in $S c_{t}$ number in section $4.1, \mathrm{~m}^{2} \mathrm{~s}^{-1}$

DE Death rate in Equations 4.2-4 and 4.2-16, $m^{-3} \mathrm{~s}^{-1}$

DIF Mass diffusivity in Equations 4.1-17 and 4.3-4, $\mathrm{m}^{2} \mathrm{~s}^{-1}$;

$f \quad$ Bin fraction in Equations 4.1-28 \& 4.2-11; Initiator efficiency in Tables 4.1-1 and 4.3-2

$\vec{F} \quad$ External forces in Equations 5.1-5, 4.2-3 and 4.3-2, $N$

$f_{B V} \quad$ Dimensionless size of daughter drops in Equation 4.2-12

$g_{t} \quad$ Gel effect coefficient in Equation 4.3-10

$\vec{g} \quad$ Gravitational acceleration, $m s^{-2}$ 
$G_{b} \quad$ Generation rate of turbulent kinetic energy due to buoyancy in Equations 4.1-22 and 4.123, $\mathrm{Kg} \mathrm{m}^{-1} \mathrm{~s}^{-3}$

$G_{k} \quad$ Generation rate of turbulent kinetic energy in Equations 4.1-22 and 4.1-23, $\mathrm{Kg} \mathrm{m}^{-1} \mathrm{~s}^{-3}$

$G_{r} \quad$ Growth rate based on particle radius in Equation $4.1-47, \mathrm{~m} / \mathrm{s}$

$G_{v} \quad$ Growth rate based on particle volume in Equation 4.1-48, $\mathrm{m}^{3} / \mathrm{s}$

$I_{\text {in }} \quad$ Initiator concentration in water in Equation 4.1-31, molecules. $\mathrm{cm}^{-3}$ water

$\overrightarrow{\vec{I}} \quad$ Unit tensor

$J \quad$ Diffusive flux in Equations 4.1-15, 4.1-16, 4.1-17 and 4.3-4, $\mathrm{kg} / \mathrm{m}^{2} \mathrm{~s}$

$K \quad$ Luo model breakage parameter in Equations 4.2-13 and 4.2-14, $\mathrm{m}^{-3} \mathrm{~s}^{-1}$;

$k_{1} \quad$ Rate constant of formation of polymer particle in section 4.1-3

$k_{2} \quad$ Rate constant for initiation and termination of particles in section 4.1-3

$k_{d} \quad$ Initiator decomposition rate coefficient in section 4.1-3, l. $s^{-1}$

$k_{\text {prop }} \quad$ Propagation rate coefficient in Tables 4.1-1 and 4.3-2, l.mol $^{-1} \cdot s^{-1}$

$K_{p q} \quad$ Exchange coefficient in Equations 4.1-5, 4.1-9 and 4.4-2, $s^{-1}$

$k_{t, 0} \quad$ Initial total termination rate coefficient in Equations 4.3-5 and 4.3-6, l.mol ${ }^{-1} . s^{-1}$

$k_{t c} \quad$ Termination by combination rate coefficient in Table 4.3-2, l.mol $^{-1} . s^{-1}$

$k_{t d} \quad$ Termination by disproportionation rate coefficient in Table 4.3-2, l.mol $\mathrm{m}^{-1} . \mathrm{s}^{-1}$

$k_{t h} \quad$ Thermal decomposition rate coefficient in Table 4.3-2, $l^{2} . \mathrm{mol}^{-2} . \mathrm{s}^{-1}$

$k_{t r f m} \quad$ Chain transfer rate to monomer constant in Table 4.3-2, $l . \mathrm{mol}^{-1} . \mathrm{s}^{-1}$

$k_{t r f s} \quad$ Chain transfer rate coefficient for solvent in Table 4.3-2, l.mol $^{-1} . s^{-1}$

$k_{\text {turb }} \quad$ Turbulence kinetic energy in Equations 4.1-18 and 4.1-22, $m^{2} s^{-2}$ 
$k_{v} \quad$ Parameter in Equation 4.1-37 and 4.1-38, $g^{-2 / 3}$

$L \quad$ Number of discrete size bins in Equation 4.1-26.

$l \quad$ Drag function in Equations 4.1-9 and 4.1-11

$M_{0} \quad$ Initial monomer concentration in Equation 4.1-37, $\mathrm{g} \mathrm{cm}^{-3}$ water

$M_{w} \quad$ Molar mass in Equation 4.3-13, gmol $^{-1}$

$m_{s} \quad$ Emulsifier micelle concentration in Equation 4.1-33 and 4.1-34, molecule.cm ${ }^{-3}$ water

$\dot{m} \quad$ Mass transfer rate in Equation 4.1-1 and 4.1-2, $\mathrm{kg} \mathrm{s}^{-1} \mathrm{~m}^{-3}$

$N \quad$ Total number density in Equation $4.2-8, m^{-3}$

n Number density function of particles with volume $V$ of droplets in Equation 4.2-8, $\mathrm{m}^{-6}$

Number of dead polymer particles in reaction kinetics in section 4.1.3; Impeller speed $N \quad$ in Equation 5.3-2, rad.s $\mathrm{s}^{-1}$

$N_{A} \quad$ Avogadro's Number in Equation 4.1-47, $\mathrm{mol}^{-1}$

$N_{T} \quad$ The total number of particles in the reactor in Equations 4.1-33 an 4.1-35

$N_{\text {aggr }} \quad$ Aggregation number of micelle in Equation 4.1-36

$\bar{n} \quad$ Number of radicals per particle in Equation 4.1-47

$N_{k}^{\prime} \quad$ Total number of particles per unit volume of reactor in Equation 4.1-26 and 4.1-27, $m^{-3}$

$\dot{N}_{T}^{\prime} \quad$ Nucleation rate in Equation 4.1-30 and 4.1-43, particles $m^{-3} s^{-1}$

$n_{k}^{\prime} \quad$ Number of particle in bin size $k$ in Equations 4.1-29 and 4.1-30, $m^{-3}$

$P \quad$ Pressure, $\mathrm{Nm}^{-2}$

$P^{\bullet} \quad$ Active polymer containing $j$ units in section 4.1.3

$Q \quad$ Volumetric flow rate in Equation 5.3-2, $\mathrm{m}^{3} \mathrm{~s}^{-1}$ 
$r \quad$ Radius of particle in Equation 4.1-47, $m$

$r_{\text {in }}$ Generation rate of initiator radical in section 4.1-3, molecules $\mathrm{cm}^{-3}$ water. $\mathrm{s}^{-1}$

$R_{\text {in }}^{\bullet} \quad$ Initiator radical concentration in water in Equation 4.1-33, molecules $\mathrm{cm}^{-3}$ water

$R^{\cdot} \quad$ Live polymer radical in sections 4.1 .3 and 4.3 .3

$R_{g} \quad$ Universal gas constant in Tables 4.1-1 and 4.3-2, 8.3 $\mathrm{J} \mathrm{mol}^{-1} \mathrm{~K}^{-1}$

S

Effective emulsifier concentration for micelle formation in Equation 4.1-37, molecules. $\mathrm{cm}^{-3}$

$\bar{S} \quad$ Source term in Equation $4.3-3, \mathrm{~kg} \cdot \mathrm{m}^{-3} \cdot \mathrm{s}^{-1}$

$S_{0} \quad S_{0}=S_{i n}-S_{C M C}$ in Equation 4.1-39, molecules. $\mathrm{cm}^{-3}$

$t \quad$ Time, $s$

$T \quad$ Inner tank diameter in section 3.1.1, $m$; temperature in Equation 4.3-8, $K$

$T_{g} \quad$ Polymer glass transition temperature in Equation 4.3-8, $K$

V Volume, $m^{3}$

$\vec{v} \quad$ Velocity in Equations $4.1-5,4.2-2$ and $4.3-2, \mathrm{~ms}^{-1}$

$\vec{v}^{\prime} \quad$ Turbulent velocity in Equations 4.1-18 and 4.1-19, $\mathrm{m} \mathrm{s}^{-1}$

$v_{f} \quad$ Specific free volume in Equations 4.3-7 and 4.3-8

$W_{i} \quad$ Mass fraction of $i^{\text {th }}$ species in Equations 4.1-15 and 4.3-3

X Monomer conversion, in Equation 4.1-42

$x_{f} \quad$ Final weight ratio of the polymer in the reactor to the total amount of monomer fed into the reactor in Equation 5.1-1

Greek Symbols

$\alpha$

Volume fraction in Equation 4.1-5, 4.1-26, 4.1-28 and 4.2-9 
$\boldsymbol{\nabla} \quad$ The Vector differential operator

$\mu \quad$ Viscosity in Equation 4.2-9 and A.2-1, $\mathrm{N} \mathrm{m}^{-2} \mathrm{~s}$

$\mu_{\text {turb }} \quad$ Turbulent viscosity in Equation 4.1-26, $\mathrm{N} \mathrm{m}^{-2} \mathrm{~s}$

$\sigma \quad$ Surface tension in Equation 4.2-14, $\mathrm{N} \mathrm{m}^{-1}$

$\sigma_{k} \quad$ Turbulent model constant in Equation 4.1-22

$\sigma_{\varepsilon} \quad$ Turbulent model constant in Equation 4.1-23

$\xi \quad$ Dimensionless eddy size in Equation 4.2-13

Breakage rate of particle size diameter $d_{i}$ in to daughter particle size diameter $D_{k}$ in

$\Omega_{B}$

Equation $4.2-13, m^{-3} s^{-1}$

$\Omega_{C}$

Coalescence rate of particles with volumes of $v_{i}$ and $v_{j}$ in Equation 4.2-15, $\mathrm{m}^{-3} \mathrm{~s}^{-1}$

$\tau \quad$ Residence time, $\min$

$\overrightarrow{\vec{\tau}} \quad$ Stress tensor in Equations $4.2-3$ and $4.3-2, \mathrm{Nm}^{-2}$

$\tau_{p} \quad$ Relaxation time in Equation 4.1-10, $s$

$\varepsilon \quad$ Parameter in Equation 4.1-35

$\varepsilon_{\text {turb }} \quad$ Turbulence dissipation rate in Equation 4.1-19, $\mathrm{m}^{2} \mathrm{~s}^{-3}$

$\rho \quad$ Density in Equation $4.1-16, \mathrm{Kg} \mathrm{m}^{-3}$

$\varphi_{c} \quad$ Critical mass fraction of monomer in the polymer particles in Equation 4.1-38

Subscripts

$B \quad$ Breakage

BV Binary Breakage 


\begin{tabular}{|c|c|}
\hline$C$ & Coalescence \\
\hline$d$ & Initiation decomposition \\
\hline$d r$ & Drift velocity \\
\hline$i$ & Index number \\
\hline$I$ & Daughter size \\
\hline II & Daughter size \\
\hline in & Initiator \\
\hline$j$ & Index number \\
\hline lift & Lift force \\
\hline$k$ & Index number \\
\hline$m$ & Monomer \\
\hline $\operatorname{mix}$ & Mixture of phases \\
\hline$p$ & Phase number \\
\hline prop & Propagation \\
\hline$P$ & Polymer \\
\hline$p q$ & Inter-phase \\
\hline$q$ & Phase number \\
\hline$r$ & Number of monomer units \\
\hline$s$ & Secondary phase \\
\hline$T$ & Total \\
\hline$t$ & Termination \\
\hline$t, 0$ & Termination without gel effect \\
\hline$t c$ & Termination by combination \\
\hline$t d$ & Termination by dissociation \\
\hline th & Thermal initiation \\
\hline
\end{tabular}




$\begin{array}{ll}\text { trfm } & \text { Chain transfer to monomer } \\ \text { trfs } & \text { Chain transfer to solvent } \\ \text { turb } & \text { Turbulent } \\ v m & \text { Virtual mass } \\ x & \text { x coordinate perpendicular to the gravitational vector } \\ y & \text { y coordinate perpendicular to the gravitational vector } \\ z & \text { z coordinate parallel to the gravitational vector }\end{array}$

Superscripts

$T \quad$ Transpose

Abbreviations

$3 D \quad$ Three-dimensions

CFD Computational fluid dynamics

CMC Critical micelle concentration

CSTR Continuous stirred tank reactor

CTA Chain Transfer agent

DSC Differential scanning calorimeter

$\mathrm{Fl} \quad$ Flow number in Equation 5.3-2

GPC Gel permeation chromatography

KPS Potassium persulphate

MMA Methyl methacrylate

$M R F \quad$ Multiple reference frame

Re Reynolds number

RMS Root mean square

rpm Revolution per minute 
$S c_{t} \quad$ Schmidt number

SDS Sodium dedecyl sulphate

SEM Scanning electron microscopy

THF Tetrahydrofuran

UDF $\quad$ User defined function 


\section{APPENDICES}

\section{A.1 User defined function for MMA emulsion Polymerization}

\#include "udf.h"

\#include "sg_pb.h"

\#include "sg_mphase.h"

DEFINE_PB_NUCLEATION_RATE(nuc_rate, cell, thread)

\{

real dn,nt,s;

real monomer_conc,polymer_conc;

real $\mathrm{I} 0=1.728 \mathrm{e} 18 ; / *$ initiator concentration molecules/cc water */

real $\mathrm{kdf}=6.65 \mathrm{e}-7 ; / *$ per second $* /$

real epsilon $=1.28 \mathrm{e} 5$;

real szero $=2.47 \mathrm{e} 19 ; / *$ molecules $/ \mathrm{cc} * /$

real ro $2=1.39 ; / *$ polymer density $1.18 * 2 \mathrm{~g} 2 / \mathrm{cm} 6$ power $2 * /$

real ini_mon $=260.41 ; / *$ initial monomer g per 1 concentration in primary phase (water and monomer) 250/0.96 $=260.41 \mathrm{~g} / \mathrm{Litre}$ or $\mathrm{kg} / \mathrm{m} 3 * /$

real $\mathrm{kv}=4.61 \mathrm{e} 15$

real rini $=22.98 \mathrm{e} 11 ; / *$ molecules $/$ sec.cc water $* /$

Thread $*$ tc $=$ THREAD_SUPER_THREAD $($ thread $) ; / *$ obtain mixture thread */

Thread $* * p t=$ THREAD_SUB_THREADS(tc); $/ *$ pointer to sub_threads */

Thread *tp = pt[P_PHASE]; /*primary phase thread */

$\mathrm{nt}=$ rini $* 130 * 60$ 
monomer_conc $=$ C_YI $($ cell,tp, 0$) * C_{-}$R $($cell, tp $) ; / *$ monomer g per litre concentration in primary phase (water and monomer) */

polymer_conc $=$ ini_mon - monomer_conc;

$/ * 0.00137 * \mathrm{~A} \mathrm{~g}$ monomer /litre water and monomer $=\mathrm{B} \mathrm{g}$ monomer/cc water $* /$

$\mathrm{s}=$ szero $-(\mathrm{kv} *$ pow $(($ polymer_conc*1.37e-6),0.66)*pow $(\mathrm{nt}, 0.33))$;

$/ * 1 \mathrm{cc}$ water $=1.37 \mathrm{e}-6 \mathrm{~m} 3$ total volume $* /$

$\mathrm{dn}=7.29 \mathrm{e} 5 *$ rini $/(1+($ epsilon* $(\mathrm{nt} / \mathrm{s})))$

$/ * \mathrm{dn}$ is in particle/(m3 total volume. second)*/

return dn;

\}

\#include "udf.h"

\#include "sg_pb.h"

\#include "sg_mphase.h"

DEFINE_PB_GROWTH_RATE(growth_rate,cell,thread,d_1)

\{

real G;

real $\mathrm{kp}=1.8 \mathrm{e} 6 ; / *$ propagation rate coefficient $\mathrm{m} 3 / \mathrm{mol} \mathrm{s} * /$

real rom $=940 . ; / *$ density of monomer g per litre */

real rop $=1180 . ; / *$ density of polymer g per litre */

real na $=6.02 \mathrm{e} 23 ; / *$ avogadro no $* /$

real nbar $=0.5 ; / *$ radical per particle $* /$

real ini_mon $=260.41 ; / *$ initial monomer kg per $\mathrm{m} 3 * /$

real $\mathrm{v}=960 . ; / *$ primary phase volume $\mathrm{m} 3 * /$ 
real monomer_conc, polymer_conc,conv,

particle_vol, particle_radius;

Thread $*$ tc $=$ THREAD_SUPER_THREAD $($ thread $) ; / *$ obtain mixture thread */

Thread $* * \mathrm{pt}=$ THREAD_SUB_THREADS(tc); $/ *$ pointer to sub_threads */

Thread *tp $=$ pt[P_PHASE $] ; \quad / *$ primary phase thread */

monomer_conc $=\mathrm{C}_{-} \mathrm{YI}(\mathrm{cell}, \mathrm{tp}, 0) * \mathrm{C} \_\mathrm{R}(\mathrm{cell}, \mathrm{tp}) ; / *$ monomer g per liter concentration in primary

phase (water and monomer) */

polymer_conc $=$ ini_mon - monomer_conc;

conv $=$ polymer_conc / ini_mon;

particle_vol $=$ polymer_conc/rop ${ }^{*} \mathrm{v}$;

particle_radius $=$ pow $($ particle_vol, 0.33$) * 0.623$;

if $($ conv $<=0.43)$

\{

$\mathrm{G}=\mathrm{kp} * \mathrm{nbar} /(\mathrm{na} * 4 * 3.14 *$ pow $($ particle_radius, 2$)) * 1.32$

\}

else

\{

if $(\operatorname{conv}=1$.

\{

$$
\mathrm{G}=0 \text {; }
$$

\}

else 


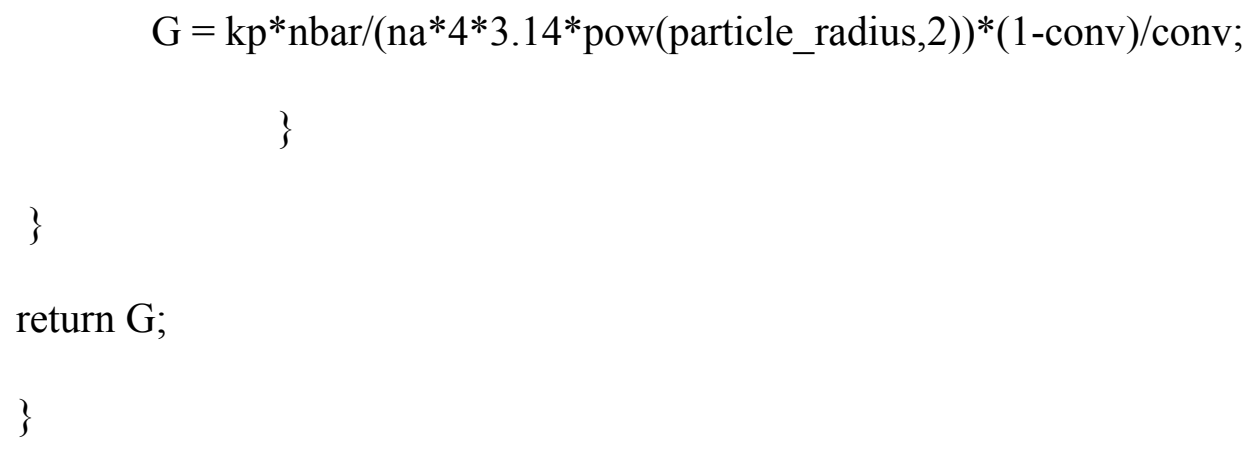

\section{A.2 Physical Properties of MMA solution polymerization}

The change in monomer viscosity with temperature can be defined according to the Equation (A.2-1) (Yaws, 1999).

$$
\begin{aligned}
& \log _{10} \mu=J_{1}+\frac{J_{2}}{T}+J_{3} T+J_{4} T^{2} \\
& J_{1}=-7.7825 \\
& J_{2}=7.3478 \times 10^{2} \\
& J_{3}=1.0258 \times 10^{-2} \\
& J_{4}=-1.1343 \times 10^{-5}
\end{aligned}
$$

Sangwai et al. (2006) developed a correlation to relate the conversion of monomer to viscosity as shown below:

$$
\log _{10}(\mu)=K^{\prime}+a \log _{10} X_{m}+\log _{10} M_{w}
$$

where $K^{\prime}=-36.4, a=12.8$ and $b=3.4$.

Also, density correlation was extracted from Baillagou and Soong as can be seen below (1985):

$$
\begin{aligned}
& \rho_{m}=966.5-1.1(T-273.15) \\
& \rho_{p}=1200 \mathrm{~kg} / \mathrm{m}^{3}
\end{aligned}
$$

where $\rho_{m}$ and $\rho_{p}$ are the densities of monomer and polymer respectively. 


\section{BIBLIOGRAPHY}

A

Adebekun, A. K., Kwalik, K. M., Schork, F. J. Steady-state multiplicity during solution polymerization of methyl methacrylate in a CSTR. Chem. Eng. Sci. 44 (1989) 2269-2281.

Afshar Ghotli, R, Raman, A. A. A., Ibrahim, S., Baroutian, S. Liquid-liquid mixing in stirred vessels: a review. Chem. Eng. Comm. 200 (2013) 595-627.

Agterof, W. G. M., Vaessen, G. E. J., Haagh, G.A.A.V., Klahn, J. K., Janssen, J. J. M. Prediction of emulsion particles sizes using a computational fluid dynamics approach. Colloids Surf B Biointerfaces. 31(2003) 141-148.

Aichele, C. P., Chapman, W.G., Rhyne, L. D., Subramani, H. J., House, W. V. Analysis of formation of water-in-oil emulsions. Energy Fuels 23 (2009) 3674-3680.

Alexopoulos, A. H., Maggioris, D., Kiparissides, C. CFD analysis of turbulence nonhomogeneity in mixing vessels A two-compartment model. Chem. Eng. Sci. 57 (2002) 17351752.

Alvarez, J., Alvarez, J., Hernandez, M. A population approach for the description of particle size distribution in suspension polymerization reactors. Chem. Eng. Sci. 49 (1994) 99-113.

ANSYS Fluent 12.0, Population balance module manual. SAS IP, Inc. (2010) PA, USA.

ANSYS Fluent 12.0, Theory Guide. ANSYS, Inc. (2009) USA.

Arratia, P.E., Kukura, J., Lacombe, J., Muzzio, F.J. Mixing of shear-thinning fluids with yield stress in stirred tanks. AIChE J. 52 (2006) 2310-2322.

Asua, J. M. Polymer Reaction Engineering. Blackwell Publishing (2007) Oxford, UK. 


\section{B}

Baillagou, P. E., Soong, D. S. Molecular weight distribution of products of free radical nonisothermal polymerization with gel effect. Simulation for polymerization of poly (methyl methacrylate). Chem. Eng. Sci. 40 (1985) 87-104.

Baldyga, J., Bourne, J. R., Pacek, A. W., Amanullah, A., Nienow, A. W. Effects of agitation and scale-up on drop size in turbulent dispersions: allowance for intermittency. Chem. Eng. Sci. 56 (2001) 3377-3385.

Baldyga, J., Podgórska, W. Drop break-up in intermittent turbulence: Maximum stable and transient sizes of drops. Can. J. Chem. Eng. 76 (1998) 456-470.

Bao, Y. , Wang, C., Huang, Z. Weng, Z. Kinetics of suspended emulsion polymerization of methyl methacrylate. Chin. J. Polym. Sci. 22 (2004) 543-548.

Bayraktar, E., Mierka, O., Platte, F., Kuzmin, D., Turek, S. Numerical aspects and implementation of population balance Equations coupled with turbulent fluid dynamics. Comput. Chem. Eng. 35 (2011) 2204- 2217.

Beuermann, S., Buback, M., Russell, G. T. Kinetics of free radical solution polymerization of methyl methacrylate over an extended conversion range. Macromol. Chem. Phys. 196 (1995) 2493-2516.

Bird, R. B., Stewart, W. E., Lightfoot, E. N. Transport phenomena. USA, John Wiley \& Sons (2007).

Blanchard, L., Hesse, J., Malhotra, S.L. Effect of molecular-weight on glass-transition by differential scanning calorimetry. Can. J. Chem. 52 (1974) 3170-3175.

Blazek, J. Computational Fluid Dynamics: Principles and Applications. 2nd ed. Amsterdam, Elsevier Inc (2005). 
Bouaswaig, A. E., Mauntz, W., Engell, S. Modeling and simulation of the particle size distribution for emulsion polymerization in a tubular reactor. $18^{\text {th }}$ European Symposium on Computer Aided Process Engineering - ESCAPE18 (2008) 647-652.

Bovey, F. A., Kolthoff, I. M., Medalia A. I., \& Meehan E. J. Emulsion polymerization. USA, Interscience Publishers Inc (1965).

Boxall, J. A., Koh, C. A., Sloan, E. D., Sum A. K., \& Wu, D. T. Measurement and calibration of droplet size distribution in water-in-oil emulsions by particle video microscope and a focused beam reflectance method. Ind. Eng. Chem. Res. 49 (2010) 1412-1418.

Brucato, A., Ciofalo, M., Crisfi, F., Micale, G. Numerical prediction of flow fields in baffled stirred vessels: a comparison of alternative modeling approaches. Chem. Eng. Sci. 53 (1998) $3653-3684$.

C

Campolo, M., Paglianti, A. Soldati, A. Fluid dynamic efficiency and scale-up of a retreated blade impeller CSTR. Ind. Eng. Chem. Res. 41 (2002) 164-172.

Chan, N., Cunningham, M. F., Hutchinson, R. Continuous controlled radical polymerization of methyl acrylate in a copper tubular reactor, Macromol. Rapid Commun. 32 (2011) 604-609.

Chatzi, E. G., Kiparissides, C. Steady-state drop-size distributions in high holdup fraction dispersion systems. AIChE J. 41 (1995) 1640-1652.

Chatzi, E. G., Boutris, C. J., Kiparissides, C. On-line monitoring of drop size distributions in agitated vessels: 1. Effects of temperature and impeller Speed. Ind. Eng. Chem. Res. 30 (1991) $536-543$.

Chen, P., Sanyal, J., Duduković, M. P. Numerical simulation of bubble column flows: Effect of different breakup and coalescence closures. Chem. Eng. Sci. 60 (2005) 1085-1101. 
Cheng, D., Cheng, J., Li, X., Wang, X., Yang, C., Mao, Z. Experimental study on gas-liquidliquid macro-mixing in a stirred tank. Chem. Eng. Sci. 75 (2012) 256-266.

Chern, C. S. Emulsion polymerization mechanisms and kinetics. Prog. Polym. Sci. 31 (2006) $443-486$.

Choi, K. Y. Analysis of steady state of free radical solution polymerization in a continuous stirred tank reactor. Polym. Eng. Sci. 26 (1986) 975-981.

Choi, Y., Lee, W. Effects of agitation in emulsion polymerization of vinyl acetate, ethylene, and N-methylol acrylamide. Ind. Eng. Chem. Res. 16 (2010) 431-436.

Congalidis, J. P., Richards, J. R. Process control of polymerization reactors : An industrial perspective. Polym. React. Eng. 6 (1998) 71-111.

Coulaloglou, C. A., Tavlarides L. L. Description of interaction processes in agitated liquid-liquid dispersions. Chem. Eng. Sci. 32 (1977) 1289-1297.

Cunningham, M.F., Geramita, K., Ma, J. W., Measuring the Effects of Dissolved Oxygen in Styrene Emulsion Polymerization. Polymer 41 (2000) 5385-5392.

\section{D}

D’Hooge, D. R., M. F. Reyniers, F. J. Stadler, B. Dervaux, C. Bailly, F.E. Du Prez, G. B. Marin, Atom transfer radical polymerization of isobornyl acrylate: a kinetic modeling study, Macromolecules 43 (2010) 8766-8781.

Deen, N.G., Solberg, T., Hjertager, B. H. Flow generated by an aerated Rushton impeller: twophase PIV experiments and numerical simulations. Can. J. Chem. Eng. 80 (2002) 1-15.

Dehnavi, M. A., Shahhosseini, S., Hashemabadi, S. H., Ghafelebashi, M. CFD based evaluation of polymer particles heat transfer coefficient in gas phase polymerization reactors. Int. Commun. Heat. Mass. 35 (2008) 1375-1379. 
Demir, M. M., Castignolles, P., Akbey, U., Wegner, G. In-situ bulk polymerization of dilute particle/MMA dispersions. Macromolecules, 40 (2007) 4190-4198.

Dimitratos, J., Elicabe, G., Georgakis, C. Control of Emulsion Polymerization Reactors. AIChE J. 40 (1994) 1993-2021.

Do Amaral, M., Arevalillo, A., Santos, J. L., Ausa, J. M. Novel insight into the mini emulsification process: CFD applied to ultrasonication. Prog. Colloid Polym. Sci. 124 (2004) 103-106.

Drew, D. A., Lahey, R. T. In particulate two phase flow. Boston, Butterworth-Heinemann (1993).

$\mathbf{E}$

Elgebrandt, R. C., Fletcher, D. F., Gomes, V. G., Romagnoli, J. A. A framework for modeling particle size effects in emulsion polymerization systems using computational fluid dynamics linked to a detailed population balance model. 16th European symposium on computer aided process engineering and 9th international symposium on process systems engineering (2006) $551-556$.

Elgebrandt, R. C., Romagnoli, J. A., Fletcher, D. F., Gomes, V. G., Gilbert, R. G. Analysis of shear-induced coagulation in an emulsion polymerization reactor using computational fluid dynamics. Chem. Eng. Sci. 60 (2005) 2005-2015.

Elghobashi, S. Particle-laden turbulent flows: direct simulation and closure models. Appl. Sci. Res. 48 (1991) 301-314.

El-Hamouz, A., Cooke, M., Kowalski, A., Sharratt, P. Dispersion of silicone oil in water surfactant solution: Effect of impeller speed, oil viscosity and addition point on drop size distribution. Chem. Eng. Process. 42 (2009) 633-642. 
Ellis, M. F., and T. W. Taylor, K. F. On-line molecular weight distribution estimation and control in batch polymerization. AIChE J. 40 (1994) 445-462.

Erdogan, S., Alpbaz, M., Karagoz, A. R. The effect of operational conditions on the performance of batch polymerization reactor. Chem. Eng. J. 86 (2002) 259-268.

Eskin, D., Derksen, J. Introduction to a Series of featured articles: Multiphase computational fluid dynamics for industrial processes. Can. J. Chem. 89 (2011) 203-205.

$\mathbf{F}$

Faldi, A., Tirrell, M. Monomer diffusion and the kinetics of methyl methacrylate radical polymerization at intermediate to high conversion. Macromolecules 27 (1994) 4184-4192.

Farzi, G., Mortezaei, M., Badiei, A. Relationship between droplet size and fluid flow characteristics in miniemulsion polymerization of methyl methacrylate. J. Appl. Polym. Sci. 120 (2011) 1591-1596.

Fathi Roudsari, S., Dhib, R., Ein-Mozaffari, F. Mixing effect on emulsion polymerization in a batch reactor, Polym. Eng. Sci. (2014a) article number: PES-13-1213.R2

Fathi Roudsari, S., Dhib, R., Ein-Mozaffari, F. Impact of impeller type on methyl methacrylate emulsion polymerization in a batch reactor, J. Appl. Polym. Sci. (2014b) 131, 40496.

Fathi Roudsari, S., Ein-Mozaffari, F., Dhib, R. Use of CFD in modeling MMA solution polymerization in a CSTR, J. Chem. Eng. 219 (2013) 429-442.

Fathi Roudsari, S., Turcotte, G., Dhib, R., Ein-Mozaffari, F. CFD modeling of the mixing of water in oil emulsions. Comput. Chem. Eng. 45 (2012) 124-136.

Feng, H., Dan, Y., Zhao, Y. A sensitive fluorescence method for monitoring the kinetics of microemulsion polymerization. Can. J. Chem. 88 (2010) 185-191.

Fleury, P. A., Meyer, T., Renken, A. Solution polymerization of methyl-methacrylate at high conversion in a recycle tubular reactor, Chem. Eng. Sci. 47 (1992) 2597-2602. 
Fogler, S. Elements of Chemical Reaction Engineering, Fourth ed. Upper Saddle River, NJ, H. Prentice Hall (2006).

Fontenot, K., Schork, F. J. Batch polymerization of methyl methacrylate in mini/macroemulsions. J. Appl. Polym. Sci. 49 (1993) 633-655.

Fox, R. O. Optimal moment sets for multivariate direct quadrature method of moments. Ind. Eng. Chem. Res. 48 (2009) 9686- 9696.

G

Ghosh, P., Gupta, S. K., Saraf, D. N. An experimental study on bulk and solution polymerization of methyl methacrylate with responses to step changes in temperature, J. Chem. Eng. 70 (1998) $25-35$.

Gilbert, R. G. Emulsion Polymerization: A Mechanistic Approach, London, Academic Press (1995).

Guo, H., Hamielec, A. E., Zhu, S. Experimental study of emulsion polymerization with crosslinking. J. Appl. Polym. Sci. 66 (1997) 935-957.

Gupta, G. N., Mandal, B. M. Solution polymerization of methyl methacrylate using trioctylmethylammonium persulfate initiator: Kinetics of polymerization and activation parameters for the primary decomposition of the initiator. J. Polym. Sci. 28 (1990) 2729-2739.

\section{$\mathbf{H}$}

Hagesaether, L., Jakobsen, H. A., Svendsen, H. F. A model for turbulent binary breakup of dispersed fluid particles. Chem. Eng. Sci. 57 (2002) 3251- 3267.

Hansen, F. K. , Ugelstad, J. Particle Nucleation in Emulsion Polymerization. I. A Theory for Homogeneous Nucleation. J. Polym. Sci. Polym. Chem. Ed. 16 (1978) 1953-1979. 
Hansen, F. K., Ugelstad, J. Particle Nucleation in Emulsion Polymerization. III. Nucleation in Systems with Anionic Emulsifier Investigated by Seeded and Unseeded Polymerization. J. Polym. Sci., Polym. Chem. 17 (1979a) 3047-3067.

Hansen, F. K., Ugelstad, J. Particle Nucleation in Emulsion Polymerization. IV. Nucleation in Monomer Droplets. J. Polym. Sci., Polym. Chem.17 (1979b) 3069-3082.

Hansen, F. K., Ugelstad, J. Particle Nucleation in Emulsion Polymerization. II. Nucleation in Emulsifier-Free Systems Investigated by Seed Polymerization. J. Polym. Sci., Polym. Chem. 17 (1979c) 3033-3045.

Harada, M., Nomura, M., Kojima, H., Eguchi, W. Nagata, S. Rate of emulsion polymerization of styrene. J. Appl. Polym. Sci. 16 (1972) 811-833.

Harvey, A. D., Rogers, S. E. Steady and unsteady computation of impeller stirred reactors. AIChE J. 42 (1996) 2701-2712.

Hayworth, C. B., Treybal, R. E. Drop formation in two-liquid-phase systems. Ind. Eng. Chem. Res. 42 (1950) 1174-1181.

Heath, A. R., Koh, P. T. L. Combined population balance and CFD modeling of particle and aggregation by polymeric flocculant. Third International Conference on CFD in the Minerals and Process Industries CSIRO, Melbourne, Australia (2003) 339-344.

Henkes, R. A. W. M., van der Flugt, F. F., Hoogendoorn, C. J. Natural convection flow in a square cavity calculated with low-Reynolds-number turbulence models. J. Int. J. Heat Mass Transfer 34 (1991) 1543-1557.

Hill, P. J., Ng, K. M. New discretization procedure for the breakage Equation. AIChE J. 41 (1995) 1204-1216. 
Hoffmann, K. A., Chiang, S. T. Computational fluid dynamics: volume III. USA, Engineering Education System (2000).

Holland, F. A., Bragg, R. In Fluid Flow for Chemical Engineers, 2nd ed. Oxford, UK, Butterworth-Heinemann (1995).

Holland, F. A., Chapman, F. S. Liquid Mixing and Processing in Stirred Tanks, New York, Reinhold (1966).

Hong, J. Effects of agitation in emulsion polymerization-kinetic and mechanistic study of coagulum. PhD Thesis, USA, Bethlehem, Lehigh University (2008).

Hosseini, A., Bouaswaig, A., Engell, S. Comparison of classical population balance models of emulsion polymerization with experimental results and a stochastic extension. Chem. Eng. Sci. 72 (2012) 179-194.

Hounslow, M. J., Ryall, R. L., Marshall, V. R. A discretized population balance for nucleation, growth, and aggregation. AIChE J. 34 (1988) 1821-1832.

\section{I}

Ishii, M., Zuber, N. Drag coefficient and relative velocity in bubbly, droplet or particulate flows. AIChE J. 25 (1979) 843-855.

$\mathbf{K}$

Kemmere, M. F., Meuldijk, J., Drinkenburg, A.A.H., German, A. L., 1999. Emulsification in batch emulsion polymerization. J. Appl. Polym. Sci. 74 (1999) 3225-3241.

Kemmere, M. F., Meuldijk, J., Drinkenburg, A. A. H., German, A. L. Rheology and flow during high solids emulsion polymerization of styrene. Polym. React. Eng. 6 (1998) 243-252.

Kemmere, M. F., Meyer, T. Supercritical Carbon Dioxide: In Polymer Reaction Engineering. Weinheim, Germany, Wiley-VCH (2005). 
Kerdouss, F., Bannari, A., Proulx, P., Bannari, R., Skrga, M., Labrecque, Y. Two-phase mass transfer coefficient prediction in stirred vessel with a CFD model. Comput. Chem. Eng. 32 (2008) 1943-1955.

Kim, J. Y., R. L. Laurence, The mixing effect on the free radical MMA solution polymerization. Korean J. Chem. Eng. 15 (1998) 273-286.

Kim, Y. H., Glasgow, L. A. Simulation of aggregate growth and breakage in stirred tanks. Ind. Eng. Chem. Res. 26 (1987)1604-1609.

Kolhapure, N. H., Fox, R. O. CFD analysis of micromixing effects on polymerization in tubular low-density polyethylene reactors. Chem. Eng. Sci. 54 (1999) 3233-3242.

Kolhapure, N. H., J. N. Tilton, C. J. Pereira, Integration of CFD and condensation polymerization chemistry for a commercial multi-jet tubular reactor. Chem. Eng. Sci. 59 (2004) $5177-5184$.

Kostoglou, M., Dovas, S., Karabelas, A. J. On the steady-state size distribution of dispersions in breakage processes. Chem. Eng. Sci. 52 (1997) 1285-1299.

Kumar, A., Gupta, R. K. Fundamentals of Polymer Engineering. New York, Marcel Dekker, Inc (2003).

Kumar, S., Ramkrishna, D. On the solution of population balance Equations by discretization-I. A fixed pivot technique, Chem. Eng. Sci. 51 (1996) 1311-1332.

$\mathbf{L}$

Laakkonen, M., Moilanen, P., Alopaeus, V., Aittamaa, J. Modelling local bubble size distributions in agitated vessels. Chem. Eng. Sci. 62 (2007) 721-740.

Launder, B. E., Spalding, D. B. The numerical computation of turbulent flows. Comput. Methods in Appl. Mech. Eng. 3 (1974) 269-289. 
Launder, B.E. Second-moment closure and its use in modeling turbulent industrial flows. International Journal for Numerical Methods in Fluids. 9 (1989) 963-985.

Lee, Y. M., Lee, J. Interactions of mixing, diffusion, and reaction in fast step growth polymerizations. Polym. Plast. Technol. Eng. 28 (1989) 151-184.

Lehr, F. Berechnen von Blasengrößenverteilungen und Strömungsfeldern in Blasensäulen. $\mathrm{PhD}$ Thesis, Germany, University of Hannover (2001).

Lepercq-Bost, E., Giorgi, M., Isambert, A., Arnaud, C. Use of the capillary number for the prediction of droplet size in membrane emulsification. J. Membr. Sci. 314 (2008) 76-89.

Lingnau, J., Meyerhoff, G. Spontaneous polymerization of methyl methacrylate. 8 . Polymerization kinetics of acrylates containing chlorine atoms. Macromolecules 17 (1984) 941945.

Litster, J. D., Smit, D. J., Hounslow, M. J. Adjustable discretization population balance for growth and aggregation. AIChE J. 41 (1995) 591-603.

Liu, Z. , Jin, T., Kind, M. Continuous polymerization of methyl methacrylate in a taylor-couette reactor. I. Influence of fluid dynamics on monomer conversion Polym. Eng. Sci. 53 (2013) 96104.

Loth, E. Quasi-steady shape and drag of deformable bubbles and drops. Int. J. Multiphas. Flow. 34 (2008) 523-546.

Louie, B. M., Carratt, G. M., Soong, D. S. Modeling the free radical solution and bulk polymerization of methyl methacrylate. J. Appl. Polym. Sci. 30 (1985) 3985-4012.

Luo, J. Y., Gosman, A. D., Issa, R. I., Middleton, J. C., Fitzgerald, M. K. Full flow field computation of mixing in baffled stirred vessels. Chem. Eng. Res. Des. 71 (1993) 342-344. 
Luo, H. Coalescence, break up and liquid circulation in bubble column reactors. PhD Thesis, Trondheim, Norway, Norwegian Institute of Technology (1993).

Luo, H., Svendsen, H. F. Theoretical model for drop and bubble breakup in turbulent dispersions. AIChE J. 42 (1996) 1225-1233.

Luo, J. Y., Issa, R. I., Gosman, A. D. Prediction of impeller induced flows in mixing vessels using multiple frames of reference, IChemE Symposium Series 136 (1994) 549-556.

M

Ma, S., Song, G., Zhong, L., Tang, G. (2010). Study on bulk polymerization of methyl methacrylate initiated by low intensity ultrasonic irradiation. J. Appl. Polym. Sci. 116 (2010) $3127-3133$.

Maggioris, D., Goulas, A., Alexopoulos, A. H., Chatzi, E. G., Kiparissides, C. Prediction of particle size distribution in suspension polymerization reactors: effect of turbulence nonhomogeneity, Chem. Eng. Sci. 55 (2000) 4611-4627.

Maggioris, D., Goulas, A., Alexopoulos, A. H., Chatzi, E. G., Kiparissides, C. Use of CFD in prediction of particle size distribution in suspension polymer reactors. In European Symposium on Computer Aided Process Engineering-8, 22 (1998) S315-S322.

Marchisio, D. L., Pikturna, J. T., Fox, R. O., Vigil, R. D. , Barresi, A. A. Quadrature method of moments for population-balance Equations. AIChE J. 49 (2003) 1266-1276.

Maschio, G., Moutier, C. Polymerization reactor: The influence of "gel effect" in batch and continuous solution polymerization of methyl methacrylate. J. Appl. Polym. Sci. 37 (1989) 825840.

Matyjaszewski, K., Davis, T. P. Handbook of Radical Polymerization, Hoboken, John Wiley \& Sons (2002). 
Mendoza Marin, F. L. ,L. M. F. Lona, M. R. Wolf Maciel, and R. M. Filho, New Emulsion polymerization Tubular Reactor with Internal Angular Baffles: Reaction Temperature Effect. J. Appl. Polym. Sci. 100 (2006) 2572-2581.

Mertoğlu, M. The Synthesis of Well-Defined Functional Homo- and Block Copolymers in Aqueous Media via Reversible Addition-Fragmentation Chain Transfer (RAFT) Polymerization. PhD Thesis, Potsdam, Universität Potsdam (2004).

Meszéna, Z. G., A. F. Johnson, Prediction of the spatial distribution of the average molecular weights in living polymerisation reactors using CFD methods. Macromol. Theory Simul. 10 (2001) 123-135.

Meyer, T. Scale-up of polymerization process: A practical example. Org. Process Res. Dev. 7 (2003) 297-302.

Milton, J. S., Arnold, J. C. Introduction to Probability and Statistics. New York, McGraw-Hill College (1995).

Ming, W., Jones, F. N., Fu, S. Synthesis of nanosize poly (methyl methacrylate) microlatexes with high polymer content by a modified microemulsion polymerization. Polym. Bull. 40 (1998) $749-756$.

Moštěk, M., Kukukova, A., Jahoda, M., Machoň, V. (2005). Comparison of Different Techniques for Modelling of Flow Field and Homogenization in Stirred Vessels. Chem. Pap. 59 (2005) $380-385$.

$\mathbf{N}$

Nagata, S. Mixing: Principles and Applications. Japan, Kodansha Ltd (1975).

Narsimhan, G., Gupta, J. P., Ramkrishna, D. A model for translational breakage probability of droplets in agitated lean liquid-liquid dispersions. Chem. Eng. Sci. 34 (1979) 257-265. 
Nere, N. K., Patwardhan, A. W., Joshi, J. B. Liquid-phase mixing in stirred vessels. Turbulent flow regime. Ind. Eng. Chem. Res. 42 (2003) 2661-2698.

Neumann, H. J., Paczynska-Lahme B. Petroleum emulsions, micro-emulsions, and micellar solutions. Prog. Colloid Polym. Sci. 77 (1988) 123-126.

Nomura, M., Harada, M. , Eguchi, W. Nagata, S. Effect of stirring on the emulsion polymerization of styrene. J. Appl. Polym. Sci. 16 (1972) 835-847.

Nomura, M., Tobita, H., Suzuki, K. Emulsion Polymerization: Kinetic and Mechanistic Aspects. Adv. Polym. Sci. 175 (2005) 1-128.

$\mathbf{O}$

Odian, G., Principles of Polymerization, second ed., Wiley Interscience, NewYork, 1981. D'Hooge, D. R., M. F. Reyniers, F. J. Stadler, B. Dervaux, C. Bailly, F. E. Du Prez, G.

Ohmura, N., Nagamitsu, M., Kondo, K., Yano, T., Usui, H. Emulsion Polymerization of Vinyl Acetate in a System of Two CSTR in Series. J. Chem. Eng. Jpn. 38 (2005) 722-726.

Ohtake, T., Hano, T., Takagi, K. Effects of viscosity on drop diameter of W/O emulsion dispersed in a stirred tank. J. Chem. Eng. Jpn. 20 (1987) 443-447.

Ohtake, T., Hano, T., Takagi, K. Analysis of water entrainment into dispersed W/O emulsion drops. J. Chem. Eng. Jpn. 21 (1988) 272-276.

Ok, T., Ookawara, S., Yoshikawa, S., Ogawa, K. Drop size distribution in liquid-liquid mixing. J. Chem. Eng. Jpn. 36 (2003) 940-945.

Okufi, S., Perez de Ortiz, E. S., Sawistowski, H. Scale-up of liquid-liquid dispersions in stirred tanks. Can. J. Chem. Eng. 68 (1990) 400-406.

Oldshue, J. Y. Fluid mixing in 1989. Chem. Eng. Prog. 85 (1989) 33-42. 
Ozdeger, E. , Sudol, E. D., El-Aasser, M.S., Klein, A., Role of mixing in copolymerizations of styrene and n-butyl acrylate. J. Appl. Polym. Sci. 69 (1998) 2277-2289.

\section{P}

Pacek, A.W., Chamsart, S., Nienow, A.W., Bakker, A. The influence of impeller type on mean drop size distribution in an agitated vessel. Chem. Eng. Sci. 54 (1999) 4211-4222.

Pakzad, L., Ein-Mozaffari, F., Chan, P. Using electrical resistance tomography and computational fluid dynamics modeling to study the formation of cavern in the mixing of pseudoplastic fluids possessing yield stress. Chem. Eng. Sci. 63 (2008) 2508 - 2522.

Paquet, D. A., Ray, W. H. Tubular reactors for emulsion polymerization: I. Experimental investigation. AIChE J. 40 (1994) 73-87.

Parker, H. Y., Westmoreland, D. G., Chang, H. R. ESR study of MMA batch emulsion polymerization in real time: Effects of particle size.Macromolecules 29 (1996) 5119-5127.

Patanakar, S. V., Spalding, D. B. A calculation procedure for heat, mass and momentum transfer in three-dimensional parabolic flows. Int. J. Heat Mass Transfer 15(1972) 1787-1806.

Patel, H., Ein-Mozaffari, F., Dhib, R. CFD analysis of mixing in thermal polymerization of styrene. Comput. Chem. Eng. 34 (2010a) 421-429.

Patel, H., Dhib, R., Ein-Mozaffari, F. Computational fluid dynamics study of a styrene polymerization reactor. Chem. Eng. Technol. 33 (2010b) 258-266.

Patruno, L. E., Dorao, C. A., Svendsen, H, F., Jakobsen, H. A. Analysis of breakage kernels for population balance modelling. Chem. Eng. Sci. 64 (2009) 501-508.

Paul, E. L., Atimeo-Obeng, V. A., Kresta, S. M. Handbook of industrial mixing- Science and Practice. Hoboken, New Jersey, John Wiley \& Sons (2004).

Penlidis, A. Polymer Reactor Design, Optimization and Control in Latex Production Technology, PhD Thesis, Hamilton, McMaster University (1986). 
Penlidis, A., MacGregor, J. F., Hamielec, A. E. Mathematical modeling of emulsion polymerization reactors. Comput. app. Polym. Lab. 20(1986) 219-240.

Pohn, J., Cunningham, M. McKenna, T.F.L. Scale-Up of Emulsion Polymerization Reactors Part II - Simulations and Interpretations, Macromol. React. Eng. 7 (2013) 393-408.

Pohn, J., Heniche, M., Fradette, L., Cunningham, M., McKenna, T. Computational analysis of mixing and scale-up in emulsion polymerization reactors. Macromol. Symp. 302 (2011) 133141.

Pohn, J., Heniche, M., Fradette, L., Cunningham, M., McKenna, T. Development of a computational framework to model the scale-up of high-solid-content polymer latex reactors. Chem. Eng. Tech. 33 (2010) 1917-1930.

Ponnuswamy, S. R., A. Penlidis, Batch solution polymerization of methyl methacrylate: Parameter estimation, Chem. Eng. J. 39 (1988) 175-183.

Poubel, W. M. , J. C.C. S. Pinto, C. E. Fontes, E. N. Souza, Analysis of mixing effects in stirred tank polymerization reactor using CFD tools, 4th Lat. Am. CFD W. Appl. Oil Gas Ind., Rio de Janeiro, Brazil, July 12-13, 2010.

$\mathbf{Q}$

Qamar, S., Warnecke, G. Solving population balance Equations for two-component aggregation by a finite volume scheme. Chem. Eng. Sci. 62 (2007) 679-693.

\section{R}

Raikar, N. B., Bhatia, S. R., Malone, M. F., Henson, M. A. Experimental studies and population balance Equation models for breakage prediction of emulsion drop size distributions. Chem. Eng. Sci. 64 (2009) 2433-2447. 
Ramkrishna, D. Population balances: Theory and applications to particulate systems in engineering. San Diego, Academic Press (2000).

Ranade, V. Computational flow modeling for chemical reactor engineering. India, Academic Press (2002).

Randolph, A. D., Larson, M. A. Theory of particulate processes: Analysis and techniques of continuous crystallization. New York, Academic Press (1971).

Rawlings, J., Ray, W. The modeling of batch and continuous emulsion polymerization reactors. parti: Model formulation and sensitivity to parameters. Polym. Eng. Sci. 28 (1988) 237-256.

Reyes, Y., Asua, J. M. (2010). Modeling multiphase latex particle equilibrium morphology. J. Polym. Sci., Part A: Polym. Chem. 48 (2010) 2579-2583.

Rudin, A., Choi, P. The Elements of Polymer Science and Engineering. USA, Academic Press (2012).

Ruiz, M. C., Lermanda, P., Padilla, R. Drop size distribution in a batch mixer under breakage conditions. Hydrometallurgy J. 63 (2002) 65-74.

\section{S}

Saeed, S., Ein-Mozaffari, F., Upreti, S. R. Using computational fluid dynamics to study the dynamic behavior of the continuous mixing of Herschel-Bulkley fluids. Ind. Eng. Chem. Res. 47 (2008) 7465-7475.

Sajjadi, S., Brooks, B.W. Semibatch emulsion polymerization of butyl acrylate. I. Effect of monomer distribution. J. Appl. Polym. Sci. 74 (1999) 3094-3110.

Sajjadi, S., Jahanzad, F. Comparative study of monomer droplet nucleation in the seeded batch and semibatch miniemulsion polymerisation of styrene. Eur. Polym. J. 39 (2003) 785-794. 
Sajjadi, S. Nanoparticle Formation by Monomer-Starved Semibatch Emulsion Polymerization. Langmuir 23 (2007) 1018-1024.

Sangwai, J. S., Saraf, D. N., Gupta, S. K. Viscosity of bulk free radical polymerizing systems under near- isothemal and non-isothermal conditions. Polymer 47 (2006) 3028-3035.

Sathyagal, A. N., Ramkrishna, D., Narsimhan, G. Droplet breakage in stirred dispersions. Breakage functions from experimental drop-size distributions. Chem. Eng. Sci. 51 (1996) 13771391.

Schiller, L., Nauman, Z. Uber die grundlegenden Berechnungen bei der Schwekraftaubereitung. Z. Ver. Dtsch. Ing. 77 (1933) 318-320.

Schmidt, A. D., Ray, W. H. The dynamic behavior of continuous polymerization reactors-I: Isothermal solution polymerization in a CSTR. Chem. Eng. Sci. 36 (1981) 1401-1410.

Schütz, S., Gorbach, G., Piesche, M. Modeling fluid behavior and droplet interactions during liquid-liquid separation in hydrocyclones. Chem. Eng. Sci. 64 (2009) 3935-3952.

Scora, M. J., Dhib, R., Penlidis, A. Free-Radical Polymerization of Methyl Methacrylate with a Tetrafunctional Peroxide Initiator. J. Polym. Sci., Part A: Polym. Chem. 42 (2004) 5647-5666.

Seda Tığlı, R., Evren, V. Synthesis and characterization of pure poly (acrylate) latexes. Prog. Org. Coat. 52 (2005) 144-150.

Selma, B., Bannari, R., Proulx, P. Simulation of bubbly flows: Comparison between direct quadrature method of moments (DQMOM) and method of classes (CM). Chem. Eng. Sci. 65 (2010) 1925-1941.

Semino, D., Ray, W.H. Control of systems described by population balance Equations-II. Emulsion polymerization with constrained control action. Chem. Eng. Sci. 50 (1995) 1825-1839. 
Serra, C., Schlatter, G., Sary, N., Schönfeld, F., Hadziioannou, G. Free radical polymerization in multi laminated microreactors: 2D and 3D multi physics CFD modeling. Microfluid. Nanofluid. 3 (2007) 451-461.

Sgard, A., Schrauwen, C., Pla, F. Theoretical developments of the tendency model to simulate and optimize batch and semibatch emulsion polymerization processes, DECHEMA Monographs 134 (1998) 339-354.

Shinnar, R. On the behaviour of liquid dispersions in mixing vessels. J. Fluid Mech. 10 (1961) $259-275$.

Shinnar, R., Church, J. M. Statistical theories of turbulence in predicting particle size in agitated dispersions. Ind. Eng. Chem. Res. 52 (1960) 253-256.

Silva, L. F. L. R., Damian, R. B., Lage P. L. C. Implementation and analysis of numerical solution of the population balance Equation in CFD packages. Comput. Chem. Eng. 32 (2008) 2933-2945.

Silva, L. F. L. R., Lage, P. L. C. Development and implementation of a polydispersed multiphase flow model in Open FOAM. Comput. Chem. Eng. 35 (2011) 2653- 2666.

Silva, L.F.L.R., Rodrigues, R.C., Mitre, J.F., Lage, P. L. C. Comparison of the accuracy and performance of quadrature-based methods for population balance problems with simultaneous breakage and aggregation. Comput. Chem. Eng. 34 (2010) 286-297.

Sjoblom, J. Encyclopaedic handbook of emulsion technology. USA, Marcel Dekker (2001).

Soh, S. K., Sundberg, D. C. Diffusion-controlled vinyl polymerization. III. Free volume parameters and diffusion-controlled propagation. J. Polym. Sci., Polym. Chem. Ed. 20 (1982) 1331-1244. 
Sood, A. Particle size distribution control in emulsion polymerization. J. Appl. Polym. Sci. 92 (2004) 2884-2902.

Soon, S. Y., Harbidge, J., Titchener-Hooker, N. J., Shamlou, P. A. Processing of emulsions using a high velocity jet device. IChemE Proc. 146 (1999) 165-176.

Spicer, P. T., Keller, W., Pratsinis, S. E. The Effect of Impeller Type on Floc Size and Structure during Shear-Induced Flocculation. J. Colloid Interface Sci. 184 (1996) 112-122.

Srilatha, C., Morab, V. V., Mundada, T. P., Patwardhan, A. W. Relation between hydrodynamics and drop size distributions in pump-mix mixer. Chem. Eng. Sci. 65 (2010) 3409-3426.

Stamatoudis, M., Tavlarides, L. L. The effect of continuous-phase viscosity on the unsteady state behavior of liquid-liquid agitated dispersions. Chem. Eng. J. 35 (2007) 137-143.

Stubbs, J. M., Sundberg, D. C. The dynamics of morphology development in multiphase latex particles. Prog. Org. Coat. 61 (2008) 156-165.

$\mathbf{T}$

Takahashi, K., Takeuchi, H. Interfacial area of liquid-liquid dispersion in a mixer-settler extraction column. In Solvent Extraction 1357-1362. Kyoto, Japan, Elsevier (1992).

Tanrisever, T., Okay, O., Sonmezoglu, I.C. Kinetics of emulisifier-free emulsion polymerization of methyl methacrylate. J. Appl. Polym. Sci. 61 (1996) 485-493.

Terrazas-Moreno, S., Flores-Tlacuahuac, A., Grossmann, I. E. Simultaneous design, scheduling, and optimal control of a methyl-methacrylate continuous polymerization reactor. AIChE J. 54 (2008) 3160-3170.

Tominagaa, Y., Stathopoulosb, T. Turbulent Schmidt numbers for CFD analysis with various types of flow field. Atmos. Environ. 41 (2007) 8091-8099. 
Tshukudu, T, Zheng, H, Hua, X, Yang, J., Tan, M., Ma, J., Sun, Y., Zhu, G. Response surface methodology approach to optimize coagulation-flocculation process using composite coagulants. Korean J. Chem. Eng. 30 (2013) 649-657.

Tulig, T. J. , Tirrell, M. On the onset of the Trommsdorff effect. Macromolecules 15 (1982) 459-463.

V

Vale, H. M., McKenna, T. F. Modeling particle size distribution in emulsion polymerization reactors, Prog. Polym. Sci. 30 (2005) 1019-1048.

Valentas, K. J., Bilous, O., Amundson, N. R. Analysis of breakage in dispersed phase systems. Ind. Eng. Chem. Fundam. 5 (1966) 271-279.

Vankova, N., Tcholakova, S., Denkov, N. D., Ivanov, I. B., Vulchev, V. D., Danner, T. Emulsification in turbulent flow 1. Mean and Maximum Drop Diameters in Inertial and Viscous Regimes. J. Colloid Interface Sci. 312 (2007) 363-380.

Verros, G. D. Achilias, D. S. Modeling gel effect in branched polymer systems: free-radical solution homopolymerization of vinyl Acetate. J. Appl. Polym. Sci. 111 (2009) 2171-2185.

Versteeg, H. K., Malalasekera, W. An introduction to computational fluid dynamics - the finite volume method. Pearson, Prentice Hall (2007).

Vivaldo-Lima, E. Hamielec, A. E., Wood, P. E. Auto-acceleration effect in free radical polymerization. A comparison of the CCS and MH models. J. Polym. React. Eng. 2 (1994) 1785.

Vivaldo-Lima, E., Wood, P. E., Hamielec, A .E., Penlidis, A. Calculation of the particle size distribution in suspension polymerization using a compartment-mixing model. Canadian J. Chem. Eng. 76 (1998) 495-505. 
Vladisavljević, G . T., Kobayashi , I., Nakajima, M. Effect of dispersed phase viscosity on maximum droplet generation frequency in micro channel emulsification using asymmetric straight-through channels. Microfluid Nanofluid 10 (2011) 1199-1209.

W

Waggoner, R.A., Blum, F. D., Mac Elroy, J. M. D. Dependence of the solvent diffusion coefficient on concentration in polymer solutions, Macromolecules 26 (1993) 6841-6848.

Walstra, P. In: Encyclopedia of Emulsion Technology, Chapter 2. New York, Dekker (1983).

Walstra, P. In: Fundamentals of Interface and Colloid Science: Soft Colloids, Chapter 8. New York, Academic Press (2005).

Wang, T., Wang, J., Jin, Y. Population Balance Model for Gas-Liquid Flows: Influence of Bubble Coalescence and Breakup Models. Ind. Eng. Chem. Res. 44 (2005) 7540-7549.

Y

Yarin, L. P., Hetsroni, G. Turbulence intensity in dilute two-phase fows-3: The particlesturbulence interaction in dilute two-phase flow. Int. J. Multiphase Flow 20 (1994) 27-44.

Yaws, C. L. Chemical Properties Handbook, fifteenth ed., New York, McGraw-Hill (1999).

Yu, Z., Li, B., Cai, M., Cao, K. Emulsion polymerization of methyl methacrylate in concentration of emulsifiers below their CMCs - polymerization rate, particle size, and particlesize distribution. J. Appl. Polym. Sci. 55 (1995) 1209-1215.

\section{$\mathbf{Z}$}

Zanfir, M., Sun, X., Gavriilidis, A. Investigation of a rotating disc reactor for acetone stripping and asymmetric transfer hydrogenation: Modelling and experiments. Chem. Eng. Sci. 62 (2007) $741-755$. 
Zucca, A., Marchisio, D. L., Barresi, A. A., Fox, R. O. Implementation of the population balance Equation in CFD codes for modelling soot formation in turbulent flames, Chem. Eng. Sci. 61 (2006) $87-95$. 


\section{Publications and Presentations}

\section{a) Publications}

1- Fathi Roudsari, S., Dhib, R., Ein-Mozaffari, F. Analysis of mixing in Emulsion Polymerization of Methyl Methacrylate, submitted to a referred journal (2014).

2- Fathi Roudsari, S., Dhib, R., Ein-Mozaffari, F. Mixing effect on emulsion polymerization in a batch reactor, Polymer Engineering Science (2014) article number: PES-13-1213.R2 (in press).

3- Fathi Roudsari, S., Dhib, R., Ein-Mozaffari, F. Impact of impeller type on methyl methacrylate emulsion polymerization in a batch reactor, Journal of Applied Polymer Science, (2014) 131, 40496.

4- Fathi Roudsari, S., Ein-Mozaffari, F., Dhib, R. Use of CFD in modeling MMA solution polymerization in a CSTR, Chemical Engineering Journal, 219 (2013) 429-442.

5- Fathi Roudsari, S., Turcotte, G., Dhib, R., Ein-Mozaffari, F. CFD modeling of the mixing of water in oil emulsions. Computers and Chemical Engineering, 45 (2012) 124-136.

(Awarded the Certificate for Most Downloaded Articles in Computers and Chemical Engineering Journal, September 2012 - August 2013)

\section{b) Oral presentations}

6- Fathi Roudsari, Ein-Mozaffari, F. Dhib, R. Impact of Mixing on the Emulsion Polymerization. North American Mixing Forum (NAMF XXIV), The Sagamore, NY, USA, (June 22-27, 2014).

\section{(Selected as a Finalist in the NAMF XXIV Student Award)}

7- Effect of Mixing Geometry and Configuration on PMMA Latex Quality in a Batch Reactor. $63^{\text {rd }}$ Canadian Chemical Engineering Conference, Fredericton, NB, (October 20-23, 2013). 
8- Fathi Roudsari, Ein-Mozaffari, F. Dhib, R. Exploring the Effect of Agitation on MMA Emulsion Polymerization $63^{\text {rd }}$ Canadian Chemical Engineering Conference, Fredericton, NB, (October 20-23, 2013).

9- Fathi Roudsari, Ein-Mozaffari, F. Dhib, R. Effect of Mixing on Polymer Quality In an Emulsion Polymerization Batch Reactor. $62^{\text {nd }}$ Canadian Chemical Engineering Conference, Vancouver BC, (October 14-17, 2012).

10- Fathi Roudsari, Ein-Mozaffari, F. Dhib, R. Using CFD Modelling to Explore the Role of Mixing and Gel Effect in Solution Polymerization. $62^{\text {nd }}$ Canadian Chemical Engineering Conference, Vancouver BC, (October 14-17, 2012).

11- Fathi Roudsari, Ein-Mozaffari, F. Dhib, R. The effect of mixing on the molecular weight and size distribution in emulsion polymerization. The $34^{\text {th }} I P R$ Symposium, Waterloo University, Waterloo (May 2, 2012).

12- Fathi Roudsari, Ein-Mozaffari, F. Dhib, R. Use of CFD for exploring the effect of mixing on MMA solution polymerization in a CSTR. The $33^{\text {rd }} I P R$ Symposium, Waterloo University, Waterloo, (May 10, 2011).

13- Fathi Roudsari, Ein-Mozaffari, F. Dhib, R. Estimation of the Droplet Size Distribution in Emulsions. $61^{\text {st }}$ Canadian Chemical Engineering Conference, Particle Technology Research Centre, London, (October 23-26, 2011).

(Won one of the Prizes in PTRC Student Oral Competition 4th Annual Symposium of Particle Technology Research Centre, Western University) 


\section{c) Poster presentations}

14- Fathi Roudsari, Ein-Mozaffari, F. Dhib, R. Effectiveness of Mixing on MMA Solution Polymerization in a CSTR. The 14th Annual Industry-University Night, The Ontario Section of Society of Plastics Engineers, Oakham house Ryerson University, (March 22, 2012).

15- Fathi Roudsari, Ein-Mozaffari, F. Dhib, R. Analysis of the Effectiveness of Mixing on MMA Solution Polymerization in a CSTR. $61^{\text {st }}$ Canadian Chemical Engineering Conference, London, (October 23-26, 2011). 